\title{
Sexual Dysfunction associated with Second-Generation Antidepressants in Patients with Major Depressive Disorder - Results from a Systematic Review with Network Meta-Analysis
}

Ursula Reichenpfader, ${ }^{1,5}$ MD, MPH; Gerald Gartlehner, ${ }^{1,2}$ MD, MPH; Laura C. Morgan, ${ }^{2}$ MA; Amy Greenblatt, ${ }^{2}$ BA; Barbara Nussbaumer, ${ }^{1}$ MSc; Richard A. Hansen, ${ }^{3} \mathrm{PhD}, \mathrm{RPh}$; Megan Van Noord, ${ }^{1,2}$ MSIS; Linda Lux, ${ }^{2}$ MPA, and Bradley N. Gaynes, ${ }^{4}$ MD, MPH

${ }^{1}$ Department for Evidence-based Medicine and Clinical Epidemiology, Krems, Austria 2 RTI International, 3040 Cornwallis Road, PO Box 12194, NC 27709

${ }^{3}$ Auburn University, Harrison School of Pharmacy, 022 James E. Foy Hall, Auburn, AL 36849

${ }^{4}$ Department of Psychiatry, University of North Carolina School of Medicine Chapel Hill, Suite 304, Room J, MacNider Building, NC 27599-7160

5 Department of Medical and Health Sciences - Division of Community Medicine, Linköping University, SE-581 83 Linköping, Sweden

This project was originally funded under Contract No. HHSA-290-2007-10056I from the Agency for Healthcare Research and Quality, U.S. Department of Health and Human Services. The authors of this manuscript are responsible for its content. Statements in the manuscript should not be construed as endorsement by the Agency for Healthcare Research and Quality or the U.S. Department of Health and Human Services.

\footnotetext{
ABSTRACT

BACKGROUND: Sexual dysfunction (SD) is prevalent in patients with major depressive disorder (MDD) and is also associated with second-generation antidepressants (SGAD) which are commonly used to treat the condition. Evidence indicates underreporting of SD in efficacy studies. SD associated with antidepressant treatment is a serious side effect that may lead to early termination of treatment and worsening of quality of life. OBJECTIVES: To systematically assess the harms of SD associated with SGAD in adult patients with MDD by drug type. METHODS: We retrieved English-language abstracts from PubMed, EMBASE, the Cochrane Library, PsycINFO, and International Pharmaceutical Abstracts from 1980 to October 2012 as well as from reference lists of pertinent review articles and grey literature searches. Two independent reviewers identified randomized controlled trials (RCTs) of at least six weeks' duration and observational studies with at least 1,000 participants. STUDY SELECTION: Reviewers abstracted data on study design, conduct, participants, interventions, outcomes and method of SD ascertainment, and rated risk of bias. A senior reviewer checked and confirmed extracted data and risk of bias ratings. ANALYSES: Random effects network meta-analysis using Bayesian methods for data from head-to-head trials and placebo-controlled comparisons; descriptive analyses calculating weighted mean rates from individual trials and observational studies. RESULTS/ SYNTHESIS: Data from sixty-three studies of low and moderate risk of bias (58 RCTs, five observational studies) with more than 26,000 patients treated with SGAD were included. Based on network meta-analyses of 66 pairwise comparisons from 37 RCTs, most comparisons showed a similar risk of sexual dysfunction among included SGAD. Credible intervals, however, were wide and included differences that would be considered clinically relevant. We observed three main patterns: Bupropion had a statistically significantly lower risk of sexual dysfunction than some other SGAD, and both escitalopram, and paroxetine showed a statistically significantly higher risk of sexual dysfunction than some other SGAD. We found reporting of harms related to sexual dysfunction inconsistent and insufficient in some trials. LIMITATIONS: Most trials were conducted in highly selected populations. Search was restricted to English-language only. CONCLUSION AND IMPLICATIONS: Because of the indirect nature of the comparisons, the often wide credible intervals, and the high variation in magnitude of
} 
outcome, we rated the overall strength of evidence with respect to our findings low. The current degree of evidence does not allow a precise estimate of comparative risk of sexual dysfunction associated with a specific antidepressant. In the absence of such evidence, clinicians need to be aware of SD as a common adverse event and should discuss patients' preferences before initiating antidepressant therapy.

\section{Introduction}

Major depressive disorder (MDD) is one of the leading causes of disability worldwide, affecting 15 percent of the population in high-income countries once in their lifetime [1]. Antidepressants were the most frequently dispensed prescription drugs in the United States (US) in 2011 accounting for $\$ 11$ billion in sales and 264 million prescriptions filled [2]. Sexual dysfunction, which can involve any or all phases of the sexual response cycle (i.e., libido, arousal, orgasm and ejaculation), is associated with both the condition and the treatments used, can affect up to 50 percent of untreated depressed patients $[3$, 4]. Particularly when treated with second-generation antidepressants (SGAD), depressed patients may experience antidepressant-induced sexual dysfunction [5].

Treatment-emergent sexual dysfunction is a frequent but often under-reported serious adverse event associated with the use of SGAD. According to the U.S. Food and Drug Administration (FDA), all adverse events resulting in a substantial disruption of a person's ability to conduct normal life functions can be considered serious adverse events [6]. Onset or worsening of sexual dysfunction as an adverse event associated with antidepressant use can result in premature discontinuation of antidepressant treatment, relapse of depression, and worsened health outcomes and quality of life [79].

Rates of treatment-emergent sexual dysfunction in depressed patients from randomized clinical trials range from 15 to 80 percent $[10,11]$. Using data from a cross-sectional study in Europe, study authors estimated the prevalence of treatment-emergent sexual dysfunction in depressed patients prescribed either a selective serotonin reuptake inhibitor or serotonin-noradrenaline reuptake inhibitors to be between 37.1 percent and 61.5 percent [12]. Evidence indicates underreporting of sexual dysfunction in efficacy studies, particularly when no targeted or structured method is used to obtain information on sexual functioning, both at baseline and throughout drug treatment [13]. In a prospective observational study, investigators observed a considerably lower incidence of antidepressant-associated sexual dysfunction when sexual dysfunction was determined by spontaneous reports of study participants alone compared to using a validated sexual function-specific instrument: nearly 80 percent of those with treatment-emergent sexual dysfunction would have gone undiagnosed if only reported spontaneously [14].

A few systematic reviews addressed the issue of sexual dysfunction associated with SGAD in patients with MDD [10,15-19]. With the exception of an updated meta-analysis by Gartlehner et al. [11], which also included data from observational studies, previous systematic reviews focused on efficacy trials. This study aims to systematically review 
and assess the comparative harms of sexual dysfunction in MDD patients treated with SGAD using data from both clinical trials and observational studies.

\section{Methods}

This systematic review updates part of a larger comparative effectiveness review on SGAD funded by and conducted for the U.S. Agency for Healthcare Research and Quality (AHRQ) [19].

\subsection{Data Sources and Study Selection}

We searched PubMed, EMBASE, PsycINFO, the Cochrane Library, and International Pharmaceutical Abstracts from 1980 to October, 2012. We used Medical Subject Headings as search terms when available or key words when appropriate. We combined terms for MDD with a list of 13 specific SGAD (bupropion, citalopram, desvenlafaxine, duloxetine, escitalopram, fluoxetine, fluvoxamine, mirtazapine, nefazodone, paroxetine, sertraline, trazodone, and venlafaxine) and their specific trade names. We limited the electronic search to "adult 19+ years," "human," and "English language." We used semiautomated manual searches of reference lists of pertinent review articles and letters to the editor employing the Scopus ${ }^{\mathrm{TM}}$ citation database (www.scopus.com) [20]. The Scientific Resource Center (SRC) searched the following sources for potentially relevant unpublished literature: the U.S. FDA website, Health Canada, Authorized Medicines for the European Union, ClinicalTrials.gov, Current Controlled Trials, Clinical Study Results, World Health Organization Clinical Trials, Conference Papers Index, National Institutes of Health RePORTER, HSRProj, Hayes, Inc. Health Technology Assessment, and the New York Academy of Medicine's Grey Literature Index. The SRC also asked pharmaceutical manufacturers to submit dossiers on completed research for each drug included in this review. We received dossiers from two firms (Astra Zeneca, London, United Kingdom [UK] and Warner Chilcott, Dublin, Ireland).

Two investigators independently reviewed abstracts and full text articles (each done by two out of the authors: UR, GG, LCM, AG, BN, RAH, MVN, LL, BNG). We excluded studies available in abstract form only. We developed eligibility criteria with respect to study design, duration, patient population, interventions, and outcomes to assess sexual dysfunction as harms associated with SGAD or as treatment-emergent sexual dysfunction in adult inpatients and outpatients with MDD. We included head-to-head RCTs of at least six weeks' duration comparing SGAD. Since head-to-head evidence was lacking for many comparisons, we also included placebo-controlled trials. We also examined data from observational studies with $\geq 1000$ study participants and follow-up of at least 12 weeks. To be eligible for inclusion, a study had to report any health outcome related to sexual dysfunction, either as an adverse event (spontaneously reported by patients, systematically elicited, openly inquired, or observed by study clinicians), or as patient-rated or expert-rated outcomes of sexual dysfunction prospectively measured by a specific, validated instrument. We excluded studies that both reviewers agreed did not meet eligibility criteria. Discrepancies were resolved by discussion between both reviewers or, when necessary, by involving a third reviewer 
(see Electronic Supplementary Material 1 for information on characteristics of included studies, and Electronic Supplementary Material 2 for information on the Update search strategy).

\subsection{Quality Assessment and Data Extraction}

Two trained reviewers (two at a time out of UR, AG, BN, MVN) independently abstracted data from each study and assigned an initial risk of bias (quality) assessment using predefined criteria based on those developed by the Cochrane Collaboration (low, moderate, and high risk of bias) [21]. To assess the risk of bias in observational studies, we used criteria outlined by Deeks and colleagues [22]. Any disagreement was resolved by consensus between the respective two reviewers. A senior reviewer (one at a time out of GG, LCM, RAH, LL, BNG) evaluated completeness of data abstraction and confirmed the quality assessment. If disagreements occurred, they were resolved by consensus. We abstracted information on study characteristics (study design, eligibility criteria), intervention (drugs, dose, duration), study participants, sample size, loss to follow-up, withdrawals because of adverse events, method of determining and reporting harms-related data and outcomes associated with sexual dysfunction. We used the Evidence-based Practice Center approach, conceptually similar to the GRADE (Grading of Recommendations Assessment, Development and Evaluation) system, to assign an overall grade for strength of evidence (low, moderate, or high strength of evidence) of the outcome [23].

\subsection{Data Synthesis and Analyses}

To be included in the quantitative analysis, studies had to provide sufficient data to calculate measures of incidence of sexual dysfunction. We recalculated rates of sexual dysfunction for each study using the number of all randomized patients as the denominator to reflect a true intention-to-treat (ITT) analysis. For statistical reasons we combined all reported subtypes of sexual dysfunction (e.g. anorgasmia, ejaculation failure, ejaculation disorder, erectile dysfunction, delayed ejaculation, abnormal orgasm, decreased libido and loss of libido) into one outcome category of sexual dysfunction. When available, sex-specific rates were abstracted.

We conducted a network meta-analysis using Bayesian methods to compare rates of sexual dysfunction between SGAD, including both head-to-head trials comparing active interventions and placebo-controlled comparisons. To be included in the network metaanalysis, RCTs had to fulfill (1) the general study eligibility criteria, and (2) the statistical conditions required for network meta-analysis (consistency, heterogeneity, geometry of treatment network). We used the methods developed and illustrated in NICE Technical Support Document 2, which details the generalized linear modeling framework for network meta-analyses of RCTs [24]. We used a random effects logistic regression model adjusting for correlations between multiple-arms within each study. Study effect and outcome effect parameters were modeled by noninformative (flat) prior distributions that were normal $(0,10000)$. For the heterogeneity of the random-effects model, we used a uniform prior distribution centered at zero with sufficiently large variance. The first 20,000 simulations were discarded to allow for model convergence 
and then a further 100,000 simulations were used in estimating the posterior probabilities. Convergence was verified by trace plots and inspection of the GelmanRubin statistic for monitored parameters. Our outcome measure was adverse events of sexual dysfunction. To assess the consistency between the different reporting methods, we conducted a sensitivity analysis, including studies where sexual dysfunction was determined only by open question or spontaneous patient reports. The network metaanalysis was performed using WinBUGS Version 1.4.3, a Bayesian software package that uses Markov chain Monte Carlo (MCMC) methods. We calculated odds ratios and 95\% credible intervals (CrI) for all possible pairwise comparisons among our drugs of interest. We analyzed RCTs reporting only male-specific rates separately from RCTs reporting total rates of sexual dysfunction. Only a small number of trials included in the network meta-analysis reported sex-specific rates. We used all data from these trials combining male and female rates into total rates along with data from trials reporting total rates of sexual dysfunction only.

We conducted descriptive analyses, when conditions to perform comparative analyses could not be met. For studies providing only sex-specific rates of sexual dysfunction, we calculated weighted mean rates and 95\% confidence intervals on sex-specific rates of sexual dysfunction pooling data from arms of both the active drug comparator and placebo-controlled trials. We calculated all descriptive analyses using StatsDirect Statistical Software, version 2.7.9 (StatsDirect, Cheshire, United Kingdom). Due to differences in study design, we could not pool rates from all observational studies and also present rates from individual studies. For both, the descriptive analyses and the network meta-analysis, we also included RCTs that did not report any baseline assessment of sexual dysfunction.

\section{Results}

Our searches identified 4,476 citations (see PRISMA flow-diagram, Fig. 1) [25] for the larger comparative effectiveness review on SGAD. We screened 1,532 full text articles for eligibility; of these, 63 studies of low and moderate risk of bias (58 RCTs, five observational studies) reporting data on any sexual dysfunction outcome or adverse event met our inclusion criteria for analysis. The majority of experimental trials were of six to eight weeks in duration.

Adverse events reporting and determination of sexual dysfunction varied widely among studies. Specific methods included prospective, systematically monitored and validated instruments to measure sexual function, rating scales, or structured clinical interviews to diagnose sexual dysfunction. Additionally, study authors relied on adverse events gathered by spontaneous patient reports, or using open questions or generic checklists by clinicians. In 22 of 58 (37.9\%) RCTs, study authors did not provide any information on the method to collect adverse event data or determine sexual dysfunction. Only 14 of $58(24.1 \%)$ RCTs reporting sexual dysfunction outcomes or adverse events reported a specific method to determine adverse events or outcomes of sexual dysfunction. In seven of 16 RCTs, study authors used a standardized validated instrument to establish sexual dysfunction at baseline and during the study period; however, they did not provide sufficient data in the published article to calculate sexual dysfunction outcomes. 
All of the observational studies with data on sexual function outcomes reported the method to ascertain sexual dysfunction or adverse event; three of the five used a validated sexual function instrument (the Changes in Sexual Functioning Questionnaire; the Arizona Sexual Experience Scale; the Psychotropic-Related Sexual Dysfunction Questionnaire).

\subsection{Evidence of risk of sexual dysfunction in patients with MDD from RCTs}

Our analyses (quantitative or descriptive) included 58 RCTs of low or moderate risk of bias with information on sexual dysfunction, representing approximately 19,000 patients treated with SGAD.

\subsubsection{Network meta-analysis}

We conducted network meta-analyses of adverse events of sexual dysfunction using data from placebo-controlled or head-to-head trials. Of the 58 RCTs fulfilling the study eligibility criteria we could finally include 37 RCTs [26-62] meeting the statistical requirements for network meta-analysis. Overall, 14,576 patients were randomly assigned to placebo or one of the eleven included SGAD drugs. Twenty-two studies were two-arm trials, eleven were three-arm trials involving two different active comparisons and placebo, and four were multi-arm trials involving two or more active compounds at various dosages and placebo. The network of all included pairwise comparisons is shown in Fig. 2.

The full model random effects network meta-analysis included 66 pairwise comparisons (55 active SGAD pairwise comparisons and 11 placebo-controlled comparisons). We found statistically significant differences in adverse events of sexual dysfunction in 14 of the pairwise active comparisons (Table 1). Most comparisons showed a similar risk of sexual dysfunction among included SGAD; however, credible intervals were wide and included differences that would be considered clinically relevant. Eight individual comparisons present a statistically significantly higher risk of sexual dysfunction of one drug over another.

Nevertheless, three main patterns emerged (see Fig. 3): (1) Bupropion had a statistically significantly lower risk of sexual dysfunction than some other SGAD (escitalopram, paroxetine, and sertraline). (2) Escitalopram showed a statistically significantly higher risk of sexual dysfunction than some other SGAD (fluoxetine, mirtazapine, and nefazodone). (3) Paroxetine had a statistically significantly higher risk of sexual dysfunction than some other SGAD (fluoxetine, mirtazapine, nefazodone, and venlafaxine).

Convergence was satisfied in the full model, but not fully satisfactory for citalopramcomparisons in the model including only trials where sexual dysfunction was spontaneously reported by patients or elicited by open question. Findings of the sensitivity analyses assessing the impact of the method used to determine sexual 
dysfunction in the network meta-analysis model were somewhat conflicting in that consistency between the different reporting methods could not always be confirmed.

Because of the indirect nature of the comparisons, the often wide credible intervals, and the high variation in magnitude of outcome, we rated the overall strength of evidence with respect to our findings low.

\subsubsection{Descriptive analysis using data of trials reporting only sex-specific rates of sexual dysfunction}

Due to reasons of heterogeneity, we did not combine direct and indirect evidence for pairwise comparisons from the trials reporting only sex-specific incidence of sexual dysfunction. Instead, we performed descriptive analysis calculating weighted mean rates pooling data both from active comparator and placebo-controlled trials. Overall, we used data from 21 RCTs [63-83] providing sex-specific rates of sexual dysfunction from 4,159 patients including six different SGAD (44 study arms: 34 active arms, 10 placebo-controlled arms).

We analyzed sex-specific rates separately and required a minimum of two trials to calculate weighted mean rates (WMR). Only trials reporting male-specific rates of sexual dysfunction provided sufficient data to allow calculation of WMR. Figure 4 summarizes by specific drug, the WMR of sexual dysfunction based on male-specific rates and 95 percent confidence intervals $(95 \% \mathrm{CI})$ in patients with MDD reported in RCTs of SGAD. Across all trials reporting male-specific rates, the WMR of sexual dysfunction was 12.3 percent ( $95 \%$ CI 8.8 to 15.8), with a range of 8.8 percent of sexual dysfunction for fluoxetine ( $95 \%$ CI 0.5 to 17.0 ) and 15.8 percent for sertraline ( $95 \%$ CI 1.2 to 30.4 ). Overall, rates of sexual dysfunction did not differ among duloxetine, escitalopram, fluoxetine, paroxetine, sertraline, and venlafaxine.

\subsection{Evidence of sexual dysfunction in patients with MDD from observational studies}

Descriptive analysis of tolerability used data from almost 7,200 study participants using SGAD (with a total of $n=10$ different drugs). We were able to include five observational studies providing data on sexual dysfunction: three prospective cohort studies $[14,84$, 85], one prescription-event monitoring database study, [86] and one cross-sectional survey [87]. Except for the study by Mackay and co-authors [86] and the one by Meijer and colleagues [84], standardized validated instruments to ascertain sexual dysfunction at baseline and throughout follow-up were used. All of the five studies were conducted in various international outpatient settings. We present crude rates and incidence density rates of individual studies separately.

Table 2 summarizes, by specific drug, the incidence and prevalence of sexual dysfunction reported in three prospective cohort studies of SGAD in patients with MDD. Due to differences in study design and patient follow-up we did not calculate pooled weighted mean rates. Instead, rates from the individual studies are shown. In two of these prospective studies $[14,85]$ incidence rates at six months of follow-up were used. 
The rates reported by Clayton et al. [87] refer to prevalence data from a cross-sectional study using data of a subsample of patients free of other possible causes of sexual dysfunction, yet with a wide range of treatment duration.

Rates of sexual dysfunction tended to be higher than those reported in RCTs. Overall, the weighted mean incidence of sexual dysfunction across all observational studies was 40.4 percent ( $95 \%$ CI $28.3 \%$ to $52.6 \%$ ). Reported rates by specific drug ranged from 7.0 percent prevalence for bupropion in a cross-sectional study [87] to a 72.7 percent sixmonth-incidence for citalopram in a prospective cohort study [14]. Both studies relied on validated instruments to ascertain sexual function, although only the study by Montejo et al. established a cohort free of sexual dysfunction at the outset [14]. Sixmonth-incidence rates tended to be higher than the prevalence rates gathered from the cross-sectional survey (citalopram $72.7 \%$ vs. $30.0 \%$, fluoxetine $57.7 \%$ vs. $23.0 \%$, paroxetine $70.7 \%$ vs. $25.0 \%$, sertraline $62.9 \%$ vs. $25.0 \%$, and venlafaxine $67.3 \%$ vs. $30.0 \%$, respectively). Duenas et al. reported a six-month incidence rate of $23.4 \%$ for duloxetine in outpatients from a prospective cohort study including only sexually active patients without sexual dysfunction at study enrolment [85].

Due to differences in study design, rates of sexual dysfunction from two additional observational studies could not be pooled with the ones from the above mentioned three prospective cohort studies. Incidence density rates, which adjust for time of exposure, reported in the individual studies were converted and are shown in table 3. From a prescription-event monitoring database study [86], adverse events of only male sexual dysfunction (impotence or ejaculation failure) were recorded showing a rate of 9.6 per 1000 person-years for nefazodone, and 30 per 1000 person-years for paroxetine. Sexual dysfunction adverse events for sertraline in a prospective observational study [84] were reported as number of first adverse events per 1000 person-years, yet study authors did not ascertain participants' baseline status of sexual function prior to antidepressant medication. Patients were followed for an average duration of 5.7 months (range one to 365 days). Reported rates were higher for men than for women (loss of libido 31/1000 person-years; ejaculation failure 14/1000 person-years; impotence 9/1000 person-years; other male sexual function disorder 4/1000 person-years; female anorgasmia 6/1000 person-years; other female sexual function disorder 3/1000 person-years).

\section{Discussion}

In this systematic review with data from sixty-three studies of low and moderate risk of bias (58 RCTs, five observational studies) with more than 26,000 patients treated with SGAD, we found some variation in sexual dysfunction associated with SGAD across drugs, yet no consistent differences between drugs. The methods used to assess adverse events varied considerably in efficacy trials with about a third of the trials included in our analysis providing no information on how harms-related data was collected or how sexual function was assessed, and only a fifth of the trials using a specific method or instrument to determine sexual dysfunction. We rated the overall strength of evidence low, indicating a low confidence that the evidence reflects the true effect. 
In our network meta-analyses, most comparisons showed a similar risk of sexual dysfunction among included SGAD. Credible intervals, however, were wide and included differences that would be considered clinically relevant. Since we conducted multiple comparisons and found differences in the method sexual dysfunction was defined and reported in individual trials, these results should be interpreted cautiously. Nevertheless, we observed three main patterns: Bupropion had a statistically significantly lower risk of sexual dysfunction than some other SGAD, and both escitalopram, and paroxetine showed a statistically significantly higher risk of sexual dysfunction than some other SGAD.

Our findings of varying rates of adverse events are consistent with previous studies. Authors of a systematic review and meta-analysis using data from 234 studies with direct and indirect comparisons of SGAD found no substantial differences in efficacy for the treatment of MDD, however, differences were found with respect to onset of action, frequency of adverse events and rates of discontinuation [11]. With respect to the observed lower risk of sexual dysfunction for bupropion, our findings are consistent with a previous systematic review and meta-analysis comparing sexual adverse events of bupropion and three SSRIs (fluoxetine, paroxetine, sertraline) which found bupropion causing significantly less sexual dysfunction than the comparator drugs while having similar effectiveness [15], a finding that was later replicated in a US-cross-sectional study [87] and by authors of a comparative effectiveness review and meta-analysis [11]. That patients treated with bupropion experience less frequent treatment-emergent sexual dysfunction has been explained with the lack of serotonergic activity of this drug (a selective norepinephrine and dopamine reuptake inhibitor). We found mirtazapine to be associated with a lower risk of sexual dysfunction in some pairwise comparisons. Mirtazapine, classified a noradrenergic and specific serotonergic antidepressant, has been described to have minimum effects on monoamine reuptake and was shown to be less likely associated with sexual adverse events compared to SSRIs (fluoxetine, paroxetine, sertraline) [17].

As to the results of the descriptive analyses, we cannot draw firm conclusions about the comparative harms of second-generation antidepressants. Using data from all trials reporting male-specific rates, rates of sexual dysfunction did not differ among included SGAD. We observed lower rates of sexual dysfunction across trials reporting only sexspecific rates as compared to those reported in observational studies. Underscoring the validity of the estimate of sexual dysfunction associated with antidepressants reported in observational studies is the use of prospectively defined populations free from sexual dysfunction at baseline and the prospective assessment of sexual function with standardized and validated instruments. Conversely, the majority of the efficacy trials included in our analysis did not include such study procedures, thus making it difficult to establish treatment-emergent sexual dysfunction.

We found reporting of adverse events related to sexual dysfunction in some of the RCTs included in our analysis inconsistent and insufficient. In the majority of trials, investigators did not specify how harms-related information was gathered: definitions of adverse events of sexual dysfunction were often not explicit and clear; also, 
investigators sometimes relied on scales to assess sexual function that lacked appropriate instrument development and validation. Additionally, authors often did not state the time frame of surveillance for adverse events thus not specifying whether recording of adverse events occurred retrospectively or prospectively; also, only collecting data on adverse event for some time after the study intervention can capture longer latency. Only rarely did authors provide information on reasons for discontinuations and withdrawals due to adverse events. In none of the included RCTs did authors report on whether attribution of a specific cause was blinded to the assigned treatment. Also, it was difficult to establish from the data of the individual studies whether or how study investigators combined data for different subsets of sexual dysfunction adverse events into one outcome measure. Also, sexual dysfunction as a harm of second-generation antidepressant treatment was chosen as a major primary outcome in only a minority of included studies and authors did not describe a-priori plan of statistical analysis. Problems of low power for uncommon events or adjustment for multiple outcomes were not addressed. Due to these shortcomings by investigators of individual trials included in our analysis, comparison of rates of sexual dysfunction, particularly from efficacy trials, must be made with great caution.

Our study has several limitations. Selection of studies was limited to English-language publications only. We did not account for observed differences in medication dosages, study duration or any confounding from use of concomitant medications or comorbid conditions potentially affecting sexual function. The majority of included RCTs were of short duration so that an estimation of long-term effects of treatment-emergent sexual dysfunction is not possible. Also, we included RCTs that did not report any baseline assessment of sexual dysfunction. Thus, the reported rates sexual dysfunction in these trials might have been inflated. Furthermore, only a small number of trials included in the network meta-analysis reported sex-specific rates so we did not perform sexspecific analyses. Although type, severity and clinical course of sexual dysfunction associated with antidepressant treatment can vary by gender [88], we were not able to assess the potential impact of gender on estimated sexual dysfunction adverse events. Results of both the network meta-analyses and the descriptive analyses should therefore be interpreted with caution. Network meta-analysis is a method that combines direct and indirect information across a network of RCTs and provides estimates of the (adverse) effect of each intervention relative to each other, whether or not they have been directly compared in trials, yet the key assumption is consistency between direct and indirect estimates of effect [89]. We cannot completely rule out that observed differences across trials might be due to violations of consistency assumptions. Still, in the absence of sufficient head-to-head evidence, network meta-analysis can serve as an additional tool to synthesize multiple treatments.

The onset of treatment-emergent sexual dysfunction or aggravation of a preexisting sexual dysfunction may add to the distress of a patient with MDD, diminish the patient's quality of life, lead to the discontinuation of antidepressant treatment and also threaten the doctor-patient relationship, particularly if the patient has not been fully informed of such adverse events of second-generation antidepressants [90]. Since evidence suggests that SGAD largely have similar efficacy, onset of action and specific adverse events 
profiles should guide a clinician's choice of a specific drug for an individual patient. Given the impact of sexual dysfunction on a patient's quality of life, clinicians should provide patients with all relevant information on possible sexual adverse events of a particular antidepressant and discuss patients' preferences and values before initiating antidepressant therapy. If patients are concerned about maintaining normal sexual functioning (e.g. younger patients), the decision of choosing an antidepressant that is less likely to be associated with sexual dysfunction should be discussed.

\section{Conclusions}

Based on the findings of this review using data from RCTs and observational studies on adverse events and second-generation antidepressants, the comparative risk of sexual dysfunction associated with a specific antidepressant cannot be precisely determined. Nevertheless, we observed three main patterns in our network meta-analysis with bupropion having a statistically significantly lower risk of sexual dysfunction than some other SGAD, and both escitalopram, and paroxetine showing a statistically significantly higher risk of sexual dysfunction than some other SGAD. Clinicians should routinely discuss the possibility of sexual dysfunction as adverse events of second-generation antidepressants and take into account patients' preferences when selecting an antidepressant and monitoring treatment. Further, we found inconsistencies and shortcomings in methods to determine and report adverse events of sexual dysfunction in many of the studies included in this review, thus potentially contributing to biased estimates. Future studies on SGAD should be adequately powered to provide complete, reliable, accurate and gender-specific information on adverse events and should be designed and conducted using systematic and valid methods to assess sexual dysfunction adverse events. Also, reporting quality of adverse events of sexual dysfunction in published trials should be improved to help researchers better appraise results of such trials and clinicians inform patients accordingly. 


\section{Acknowledgements:}

No sources of funding were used to assist in the preparation of this systematic review. However, this review updates part of a larger comparative effectiveness review on SGAD [19], which was funded by and conducted for the U.S. Agency for Healthcare Research and Quality, U.S. Department of Health and Human Services under Contract No. HHSA290-2007-10056I.

Amy Greenblatt has participated in research funded by the US Agency for Healthcare Research and Quality during the conduct of the study. Richard Hansen has received consulting fees from Daiichi Sankyo and Novartis for studies on unrelated topics, and has served as an expert witness for Allergan for Botox drug safety. Ursula

Reichenpfader, Gerald Gartlehner, Laura C. Morgan, Barbara Nussbaumer, Megan Van Noord, Linda Lux, and Bradley N. Gaynes have no conflicts of interest that are directly relevant to the content of this study.

Network meta-analyses were conducted by Tania Wilkins, PhD (The University of North Carolina at Chapel Hill, Gillings School of Global Public Health Biostatistics, 3101 McGavran-Greenberg Hall, Chapel Hill, NC 27599-7420). 
Fig. 1 Flow diagram: summary of evidence search and selection.
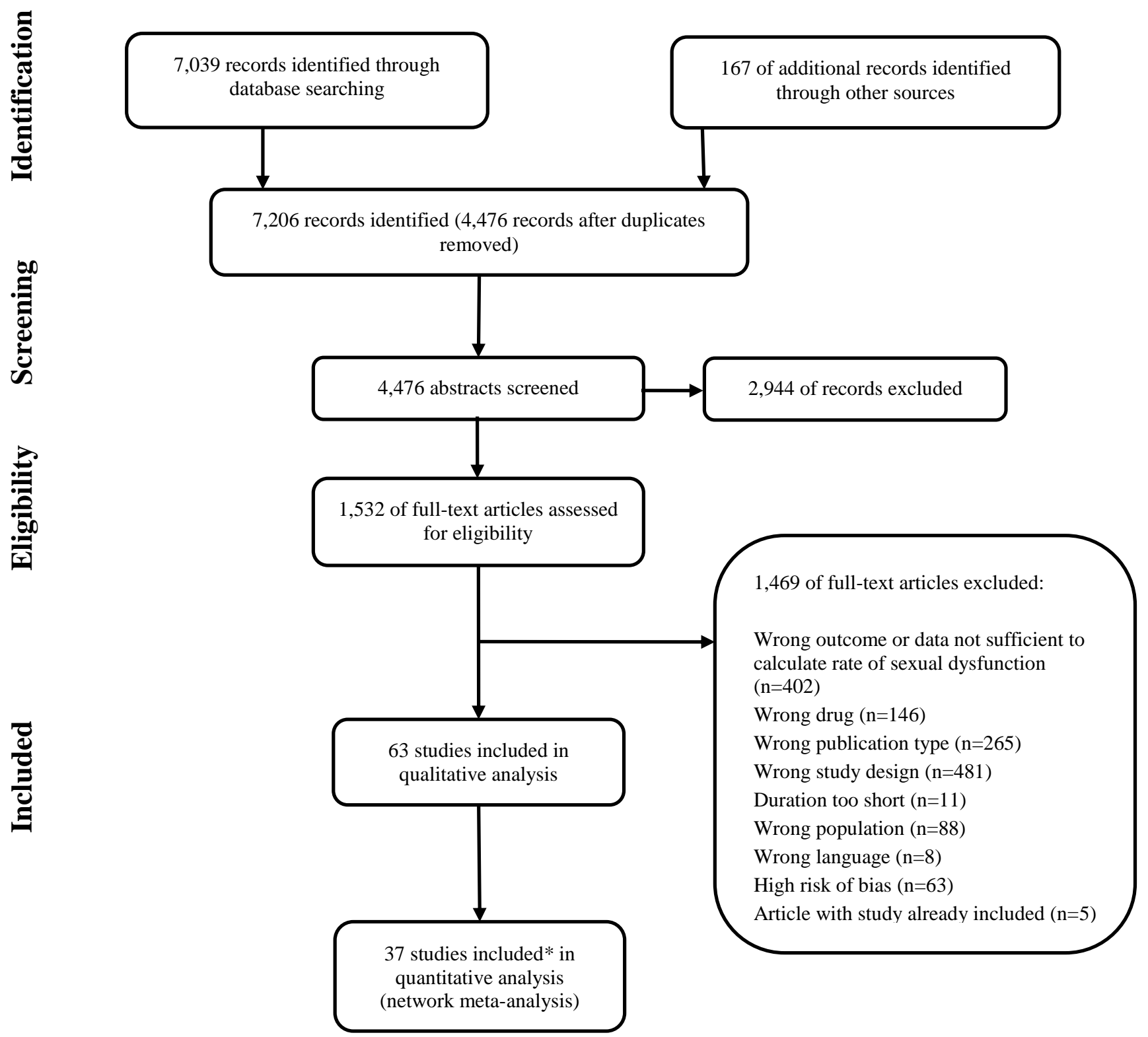

${ }^{*}$ RCTs were included in the network meta-analysis if they fulfilled (i) general study eligibility criteria, and (ii) statistical requirements for network meta-analysis. RCTs randomized controlled trials 
Fig. 2 Network of all included comparisons for the network meta-analysis of sexual dysfunction associated with second-generation antidepressants.

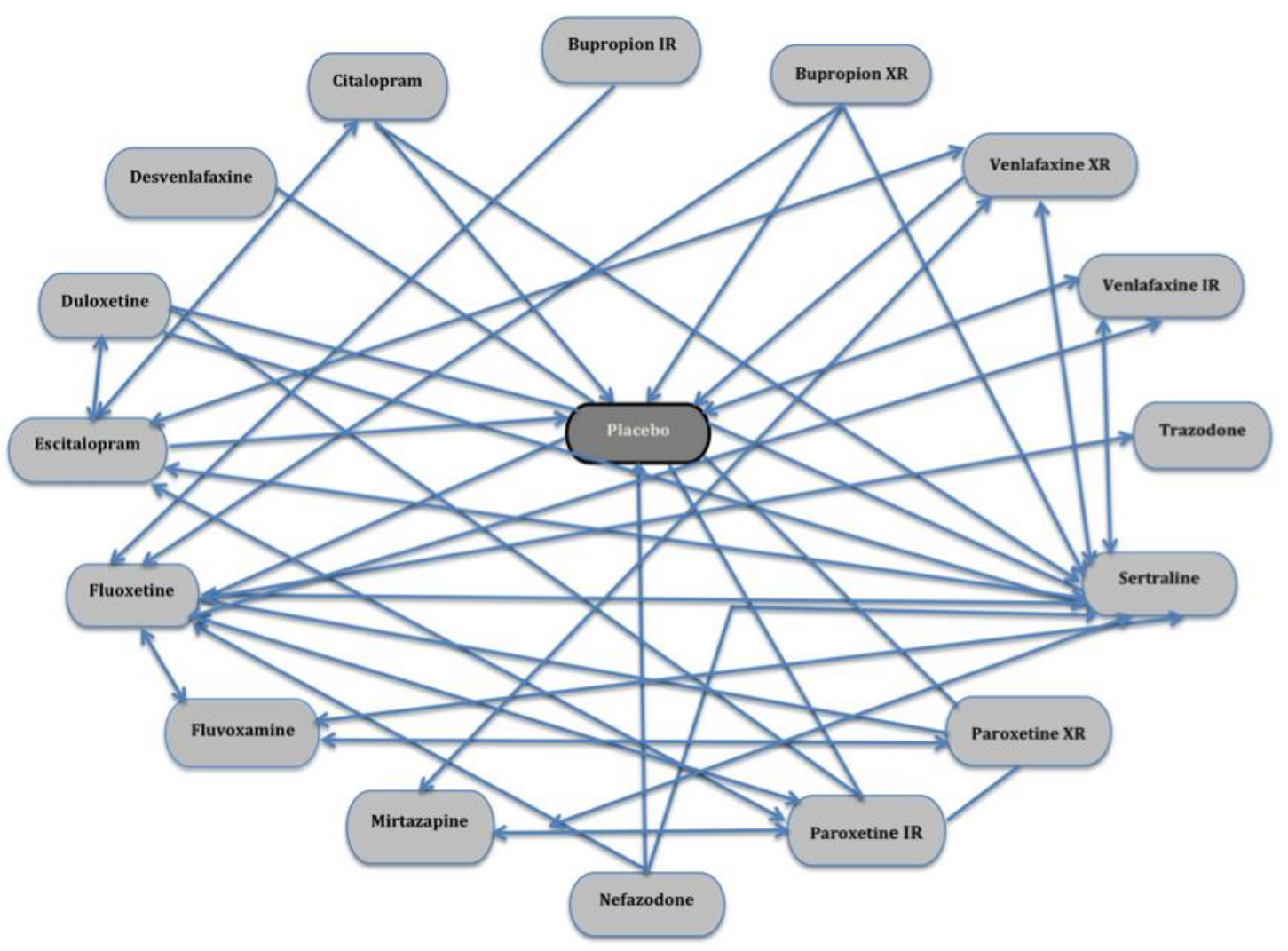

$I R$ : immediate release; $X R$ : extended release 
Fig. 3 Results of network meta-analysis of sexual dysfunction for selected pairwise second-generation antidepressants comparisons, odds ratios.

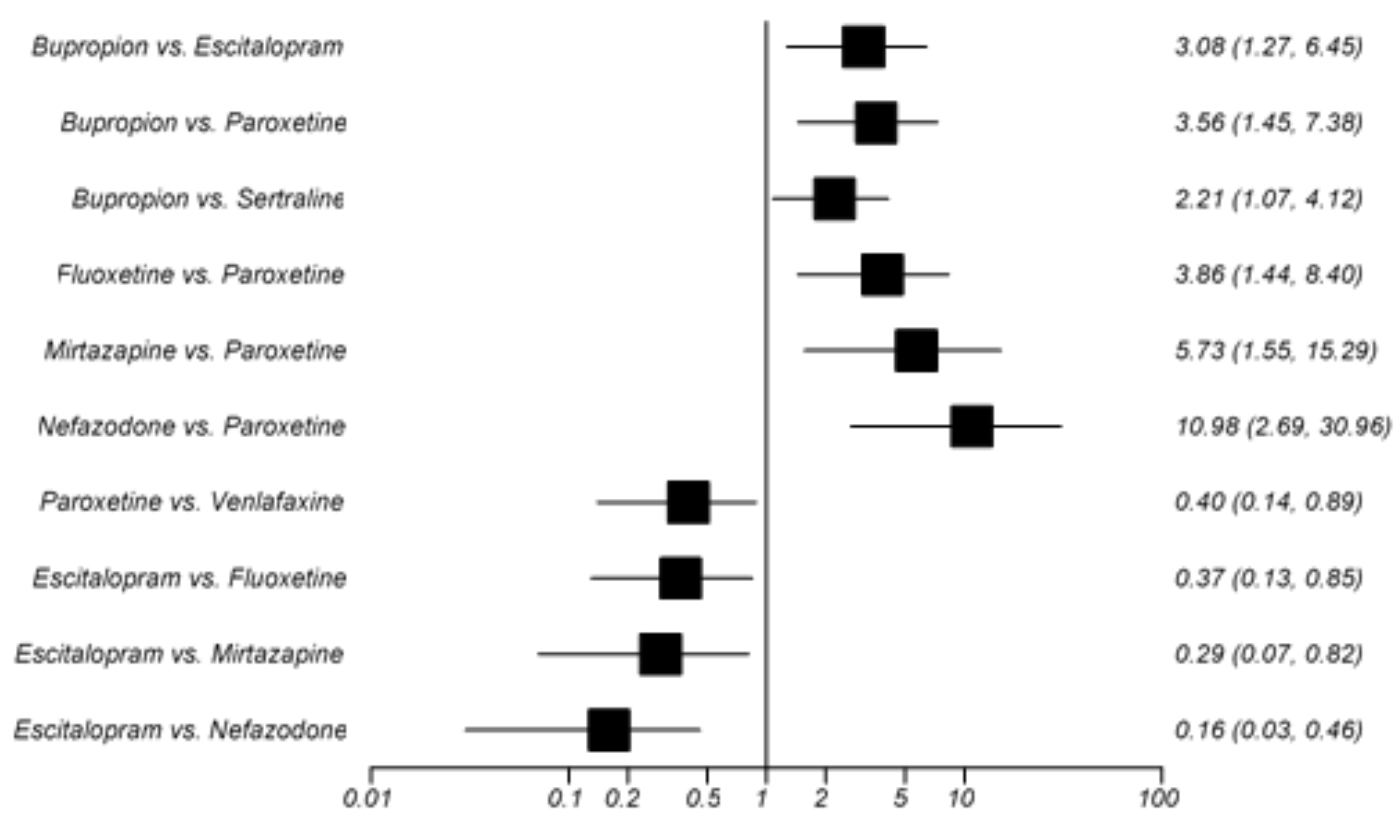

Higher rate of SD for first drug** Higher rate of SD for second drug*

* Odds ratios < 1 indicate a higher rate of sexual dysfunction of the drug listed on the left-hand side ('first drug'); ** Odds ratios $>1$ indicate a higher rate of sexual dysfunction of the drug listed on the right-hand side ('second drug'); selected results from full model of random effects network meta-analysis. $S D$ sexual dysfunction 
Fig. 4 Male-specific weighted mean rates of sexual dysfunction from individual randomized controlled trials

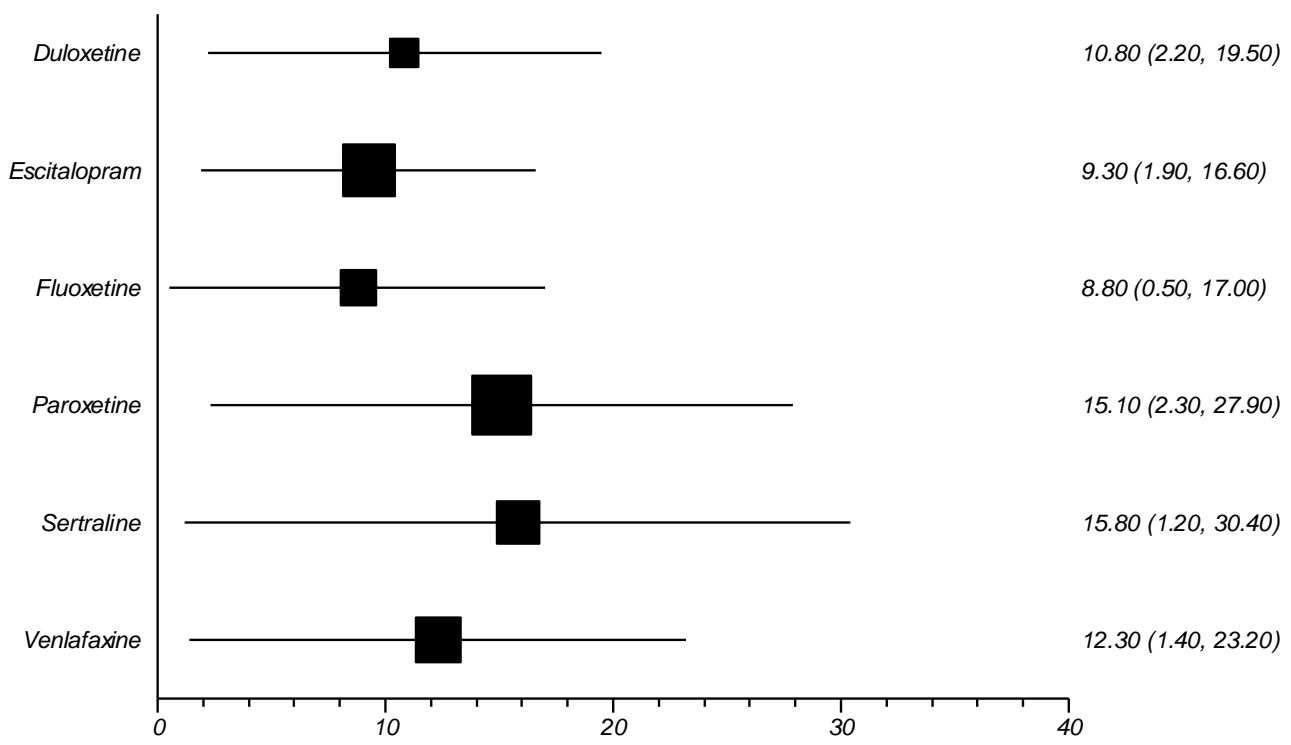

Data are presented as \% (95\% confidence intervals). Weighted mean rates (\%) calculated using drug-specific rates of sexual dysfunction from individual randomized controlled trials pooling data from active-comparator trials and placebocontrolled trials. Rates were calculated only if data from at least two trials were available (only male-specific rates could be calculated; we did not use data from a small RCT for desvenlafaxine and trazodone, respectively). Comparisons across drugs must be made cautiously: method and extent of adverse event assessment differed across studies. 
Table 1 Odds ratio of sexual dysfunction (95\% credible intervals for pairwise comparisons, mixed-treatment comparison)a

\begin{tabular}{|c|c|c|}
\hline Pairwise comparisons & $\begin{array}{l}\text { OR of sexual } \\
\text { dysfunctiona }^{\mathrm{a}}\end{array}$ & (95\% CrI) \\
\hline Bupropion vs. Citalopram & 2.94 & $(0.81-7.90)$ \\
\hline Bupropion vs. Duloxetine & 2.21 & $(0.88-4.67)$ \\
\hline Bupropion vs. Escitalopram & 3.08 & $(1.27-6.45)$ \\
\hline Bupropion vs. Fluoxetine & 1.02 & $(0.42-2.11)$ \\
\hline Bupropion vs. Fluvoxamine & 1.35 & $(0.24-4.44)$ \\
\hline Bupropion vs. Mirtazapine & 0.82 & $(0.20-2.29)$ \\
\hline Bupropion vs. Nefazodone & 0.45 & $(0.10-1.29)$ \\
\hline Bupropion vs. Paroxetine & 3.56 & $(1.45-7.38)$ \\
\hline Bupropion vs. Sertraline & 2.21 & $(1.07-4.12)$ \\
\hline Bupropion vs. Venlafaxine & 1.30 & $(0.47-2.93)$ \\
\hline Citalopram vs. Duloxetine & 0.96 & $(0.26-2.51)$ \\
\hline Citalopram vs. Escitalopram & 1.31 & $(0.41-3.15)$ \\
\hline Citalopram vs. Fluoxetine & 0.46 & $(0.11-1.30)$ \\
\hline Citalopram vs. Fluvoxamine & 0.59 & $(0.08-2.16)$ \\
\hline Citalopram vs. Mirtazapine & 0.36 & $(0.06-1.17)$ \\
\hline Citalopram vs. Nefazodone & 0.20 & $(0.03-0.66)$ \\
\hline Citalopram vs. Paroxetine & 1.57 & $(0.41-4.13)$ \\
\hline Citalopram vs. Sertraline & 0.96 & $(0.30-2.32)$ \\
\hline Citalopram vs. Venlafaxine & 0.57 & $(0.14-1.60)$ \\
\hline Duloxetine vs. Escitalopram & 1.50 & $(0.68-2.93)$ \\
\hline Duloxetine vs. Fluoxetine & 0.52 & $(0.18-1.18)$ \\
\hline Duloxetine vs. Fluvoxamine & 0.67 & $(0.12-2.17)$ \\
\hline Duloxetine vs. Mirtazapine & 0.41 & $(0.10-1.13)$ \\
\hline Duloxetine vs. Nefazodone & 0.22 & $(0.05-0.64)$ \\
\hline Duloxetine vs. Paroxetine & 1.72 & $(0.81-3.20)$ \\
\hline Duloxetine vs. Sertraline & 1.11 & $(0.48-2.25)$ \\
\hline Duloxetine vs. Venlafaxine & 0.65 & $(0.22-1.51)$ \\
\hline Escitalopram vs. Fluoxetine & 0.37 & $(0.13-0.85)$ \\
\hline Escitalopram vs. Fluvoxamine & 0.48 & $(0.08-1.58)$ \\
\hline Escitalopram vs. Mirtazapine & 0.29 & $(0.07-0.82)$ \\
\hline Escitalopram vs. Nefazodone & 0.16 & $(0.03-0.46)$ \\
\hline Escitalopram vs. Paroxetine & 1.26 & $(0.50-2.58)$ \\
\hline Escitalopram vs. Sertraline & 0.79 & $(0.39-1.54)$ \\
\hline Escitalopram vs. Venlafaxine & 0.47 & $(0.16-1.08)$ \\
\hline Fluoxetine vs. Fluvoxamine & 1.49 & $(0.24-5.07)$ \\
\hline Fluoxetine vs. Mirtazapine & 0.89 & $(0.20-2.60)$ \\
\hline Fluoxetine vs. Nefazodone & 0.49 & $(0.10-1.50)$ \\
\hline Fluoxetine vs. Paroxetine & 3.86 & $(1.44-8.40)$ \\
\hline Fluoxetine vs. Sertraline & 2.44 & $(0.94-5.26)$ \\
\hline Fluoxetine vs. Venlafaxine & 1.41 & $(0.47-3.30)$ \\
\hline Fluvoxamine vs. Mirtazapine & 0.96 & $(0.12-3.60)$ \\
\hline Fluvoxamine vs. Nefazodone & 0.52 & $(0.06-2.01)$ \\
\hline Fluvoxamine vs. Paroxetine & 4.08 & $(0.81-12.73)$ \\
\hline Fluvoxamine vs. Sertraline & 2.59 & $(0.53-7.98)$ \\
\hline Fluvoxamine vs. Venlafaxine & 1.54 & $(0.25-5.29)$ \\
\hline Mirtazapine vs. Nefazodone & 0.73 & $(0.11-2.56)$ \\
\hline Mirtazapine vs. Paroxetine & 5.73 & $(1.55-15.29)$ \\
\hline Mirtazapine vs. Sertraline & 3.62 & $(1.01-9.59)$ \\
\hline Mirtazapine vs. Venlafaxine & 2.03 & $(0.57-5.31)$ \\
\hline Nefazodone vs. Paroxetine & 10.98 & $(2.69-30.96)$ \\
\hline Nefazodone vs. Sertraline & 6.89 & $(1.81-18.89)$ \\
\hline Nefazodone vs. Venlafaxine & 4.11 & $(0.84-12.70)$ \\
\hline Paroxetine vs. Sertraline & 0.68 & $(0.30-1.35)$ \\
\hline Paroxetine vs. Venlafaxine & 0.40 & (0.14-0.89) \\
\hline Sertraline vs. Venlafaxine & 0.61 & $(0.25-1.24)$ \\
\hline
\end{tabular}

a Relative treatment effect of each treatment relative to reference comparator expressed as OR (with $95 \% \mathrm{CrI}$ ). An OR $<1$ indicates a higher rate of SD of the drug listed on the left-hand side ('first drug'); an OR >1 indicates a higher rate of SD of the drug listed on the right-hand side ('second drug'). Method and extent of adverse event assessment differed across studies; comparisons across drugs must be made cautiously. Based on random-effects network meta-analysis using Bayesian methods. $C r I$ credible interval, $O R$ odds ratio, $S D$ sexual dysfunction 
Table 2 Prevalence/Incidence of sexual dysfunction from three observational studies (crude rates as percentages from individual studies)

\begin{tabular}{|c|c|c|c|}
\hline Drug & $\begin{array}{l}\text { Sexual dysfunction } \\
\text { (mean percentage) }\end{array}$ & Study design & $\mathrm{N}$ included in analysis \\
\hline Bupropion & 7.0 & cross-sectional surveya & 45 \\
\hline \multirow[t]{2}{*}{ Citalopram } & 30.0 & cross-sectional surveya & 83 \\
\hline & 72.7 & prospective studyb & 66 \\
\hline Duloxetine & 23.4 & prospective studyc & 406 \\
\hline \multirow[t]{2}{*}{ Fluoxetine } & 23.0 & cross-sectional surveya & 245 \\
\hline & 57.7 & prospective studyb & 279 \\
\hline Fluvoxamine & 62.3 & prospective studyb & 77 \\
\hline Mirtazapine & 24.4 & prospective studyb & 49 \\
\hline Nefazodone & 8.0 & prospective studyb & 50 \\
\hline \multirow[t]{3}{*}{ Paroxetine } & 25.0 & cross-sectional surveya & 159 \\
\hline & 70.7 & prospective studyb & 208 \\
\hline & 25.0 & cross-sectional surveya & 161 \\
\hline Sertraline & 62.9 & prospective studyb & 159 \\
\hline \multirow[t]{2}{*}{ Venlafaxine } & 30.0 & cross-sectional surveya & 70 \\
\hline & 67.3 & prospective studyb & 55 \\
\hline
\end{tabular}

$M D D$ major depressive disorder, $S D$ sexual dysfunction,

a Prevalence rates; patients with MDD; subsample with patients free of other possible causes of SD; length of treatment varied (1\% < a week; $24 \%$ more than a week but less than 3 months; $17 \%$ more than 6 months but less than 12 months; $28 \%$ one to three years; $12 \%$ more than 3 years);[87]

b Incidence rates calculated at 6 months of follow-up in participants free of SD prior to antidepressant medication; [14]

c Incidence rates calculated at 6 months of follow-up in sexually active patients without SD at study enrolment; [85] 
Table 3 Incidence density rate of sexual dysfunction from two observational studies

\begin{tabular}{|c|c|c|c|}
\hline Drug & $\begin{array}{l}\text { Sexual dysfunction (incidence } \\
\text { density rates) }\end{array}$ & Study design & $\begin{array}{l}\mathrm{N} \text { included in } \\
\text { analysis }\end{array}$ \\
\hline Nefazodone & 9.6/1000 PY & $\begin{array}{l}\text { prescription-event monitoring, } \\
\text { database studya; only male SD } \\
\text { (impotence or ejaculation } \\
\text { failure), } 1 \text { study }\end{array}$ & 4418 \\
\hline Paroxetine & $30 / 1000$ PY & $\begin{array}{l}\text { prescription-event monitoring, } \\
\text { database studya; only male SD } \\
\text { (impotence or ejaculation } \\
\text { failure), } 1 \text { study }\end{array}$ & 4373 \\
\hline Sertraline & $\begin{array}{l}\text { loss of libido } 31 / 1000 \mathrm{PY} \\
\text { ejaculation failure } 14 / 1000 \mathrm{PY} \\
\text { impotence } 9 / 1000 \mathrm{PY} \text {; other } \\
\text { sexual function disorder (male) } \\
4 / 1000 \mathrm{PY} \text {; anorgasmia } \\
\text { (female) 6/1000 PY; other } \\
\text { sexual function disorder } \\
\text { (female) 3/1000 PY }\end{array}$ & $\begin{array}{l}\text { prospective observational } \\
\text { studyb, } 1 \text { study }\end{array}$ & 659 \\
\hline
\end{tabular}

$P Y$ person years, $S D$ sexual dysfunction

a Unclear whether study participants were free of SD prior to antidepressant medication; SD recorded in patients record after the date of first prescription; [86]

b Unclear whether study participants were free of SD prior to antidepressant medication; SD adverse events were ascertained by open question by clinician; start and stop dates of the events and assessment of severity ('mild', 'moderate' or 'severe') were recorded; patients were followed for an average duration of 5.7 months (range 1-365 days) [84] 


\section{References}

1. Bromet E, Andrade LH, Hwang I, Sampson NA, Alonso J, de Girolamo G, et al. Crossnational epidemiology of DSM-IV major depressive episode. BMC medicine. 2011;9:90.

2. Lindsley CW. The top prescription drugs of 2011 in the United States: antipsychotics and antidepressants once again lead CNS therapeutics. ACS chemical neuroscience. 2012 Aug 15;3(8):630-1.

3. Angst J. Sexual problems in healthy and depressed persons. Int Clin Psychopharmacol. 1998 Jul;13 Suppl 6:S1-4.

4. Kennedy SH, Dickens SE, Eisfeld BS, Bagby RM. Sexual dysfunction before antidepressant therapy in major depression. J Affect Disord. 1999 Dec;56(2-3):201-8. 5. Rothschild AJ. Sexual side effects of antidepressants. J Clin Psychiatry. 2000;61 Suppl 11:28-36.

6. U.S. Department of Health and Human Services, Food and Drug Administration. Guidance for Industry and Investigators. Safety Reporting Requirements for INDs and BA/BE Studies. Rockville, MD: U.S. Department of Health and Human Services: 2012. 7. Gregorian RS, Golden KA, Bahce A, Goodman C, Kwong WJ, Khan ZM. Antidepressant-induced sexual dysfunction. Ann Pharmacother. 2002 Oct;36(10):157789.

8. Rosenberg KP, Bleiberg KL, Koscis J, Gross C. A survey of sexual side effects among severely mentally ill patients taking psychotropic medications: impact on compliance. J Sex Marital Ther. 2003 Jul-Aug;29(4):289-96.

9. Clayton AH, Balon R. The impact of mental illness and psychotropic medications on sexual functioning: the evidence and management. The journal of sexual medicine. 2009 May;6(5):1200-11; quiz 12-3.

10. Serretti A, Chiesa A. Treatment-emergent sexual dysfunction related to antidepressants: a meta-analysis. J Clin Psychopharmacol. 2009 Jun;29(3):259-66. 11. Gartlehner G, Hansen RA, Morgan LC, Thaler K, Lux L, Van Noord M, et al. Comparative benefits and harms of second-generation antidepressants for treating major depressive disorder: an updated meta-analysis. Ann Intern Med. 2011 Dec 6;155(11):772-85.

12. Williams VS, Edin HM, Hogue SL, Fehnel SE, Baldwin DS. Prevalence and impact of antidepressant-associated sexual dysfunction in three European countries: replication in a cross-sectional patient survey. Journal of psychopharmacology (Oxford, England). 2010 Apr;24(4):489-96.

13. Kennedy SH, Eisfeld BS, Dickens SE, Bacchiochi JR, Bagby RM. Antidepressantinduced sexual dysfunction during treatment with moclobemide, paroxetine, sertraline, and venlafaxine. J Clin Psychiatry. 2000 Apr;61(4):276-81.

14. Montejo AL, Llorca G, Izquierdo JA, Rico-Villademoros F. Incidence of sexual dysfunction associated with antidepressant agents: a prospective multicenter study of 1022 outpatients. Spanish Working Group for the Study of Psychotropic-Related Sexual Dysfunction. J Clin Psychiatry. 2001;62 Suppl 3:10-21.

15. Nieuwstraten CE, Dolovich LR. Bupropion versus selective serotonin-reuptake inhibitors for treatment of depression. Ann Pharmacother. 2001 Dec;35(12):1608-13. 16. Cipriani A, Purgato M, Furukawa TA, Trespidi C, Imperadore G, Signoretti A, et al. Citalopram versus other anti-depressive agents for depression. Cochrane database of systematic reviews (Online). 2012;7:CD006534.

17. Watanabe N, Omori IM, Nakagawa A, Cipriani A, Barbui C, Churchill R, et al. Mirtazapine versus other antidepressive agents for depression. Cochrane database of systematic reviews (Online). 2011 (12):CD006528.

18. Cipriani A, La Ferla T, Furukawa TA, Signoretti A, Nakagawa A, Churchill R, et al. Sertraline versus other antidepressive agents for depression. Cochrane database of systematic reviews (Online). 2010 (4):CD006117.

19. Gartlehner G, Hansen RA, Morgan LC, Thaler K, Lux LJ, Van Noord M, et al. SecondGeneration Antidepressants in the Pharmacologic Treatment of Adult Depression: An Update of the 2007 Comparative Effectiveness Review. Rockville MD, 2011 Dec. 
20. Chapman A, Morgan LC, Gartlehner G. Semi-automating the manual literature search for systematic reviews increases efficiency. Health Information \& Libraries Journal 2009;27(1):22-7.

21. Higgins JPT, Altman DG, Sterne J. Chapter 8: Assessing risk of bias in included studies. In: Higgins JPT, S G, editors. Cochrane Handbook for Systematic Reviews of Interventions Version 510 [updated March 2011]: The Cochrane Collaboration; 2011. 22. Deeks JJ, Dinnes J, D'Amico R, Sowden AJ, Sakarovitch C, Song F, et al. Evaluating non-randomised intervention studies. Health Technol Assess. 2003;7(27):iii-x, 1-173. 23. Owens DK, Lohr KN, Atkins D, Treadwell JR, Reston JT, Bass EB, et al. AHRQ series paper 5: grading the strength of a body of evidence when comparing medical interventions--agency for healthcare research and quality and the effective health-care program. J Clin Epidemiol. 2010 May;63(5):513-23.

24. Dias S, Welton NJ, Sutton AJ, Ades AE. NICE DSU Technical Support Document 2: A Generalised Linear Modelling Framework for Pairwise and Network Meta-Analysis of Randomised Controlled Trials. . 2011 (last updated March 2013); available from http://www.nicedsu.org.uk.

25. Liberati A, Altman DG, Tetzlaff J, Mulrow C, Gøtzsche PC, Ioannidis JPA, et al. The PRISMA Statement for Reporting Systematic Reviews and Meta-Analyses of Studies That Evaluate Health Care Interventions: Explanation and Elaboration. PLoS Med. 2009;6(7):e1000100.

26. Alvarez E, Perez V, Dragheim M, Loft H, Artigas F. A double-blind, randomized, placebo-controlled, active reference study of Lu AA21004 in patients with major depressive disorder. Int J Neuropsychopharmacol. 2012 Jun;15(5):589-600.

27. Behnke K, Sogaard J, Martin S, Bauml J, Ravindran AV, Agren H, et al. Mirtazapine orally disintegrating tablet versus sertraline: a prospective onset of action study. J Clin Psychopharmacol. 2003 Aug;23(4):358-64.

28. Benkert 0, Szegedi A, Kohnen R. Mirtazapine compared with paroxetine in major depression. J Clin Psychiatry. 2000 Sep;61(9):656-63.

29. Clayton AH, Croft HA, Horrigan JP, Wightman DS, Krishen A, Richard NE, et al. Bupropion extended release compared with escitalopram: effects on sexual functioning and antidepressant efficacy in 2 randomized, double-blind, placebo-controlled studies. J Clin Psychiatry2006. p. 736-46.

30. Clayton A, Kornstein S, Prakash A, Mallinckrodt C, Wohlreich M. Changes in sexual functioning associated with duloxetine, escitalopram, and placebo in the treatment of patients with major depressive disorder. The journal of sexual medicine. 2007 Jul;4(4 Pt 1):917-29.

31. Coleman CC, King BR, Bolden-Watson C, Book MJ, Segraves RT, Richard N, et al. A placebo-controlled comparison of the effects on sexual functioning of bupropion sustained release and fluoxetine. Clin Ther. 2001 Jul;23(7):1040-58.

32. Coleman CC, Cunningham LA, Foster VJ, Batey SR, Donahue RM, Houser TL, et al. Sexual dysfunction associated with the treatment of depression: a placebo-controlled comparison of bupropion sustained release and sertraline treatment. Ann Clin Psychiatry. 1999 Dec;11(4):205-15.

33. Croft H, Settle E, Jr., Houser T, Batey SR, Donahue RM, Ascher JA. A placebocontrolled comparison of the antidepressant efficacy and effects on sexual functioning of sustained-release bupropion and sertraline. Clin Ther. 1999 Apr;21(4):643-58.

34. Delgado PL, Brannan SK, Mallinckrodt CH, Tran PV, McNamara RK, Wang F, et al. Sexual functioning assessed in 4 double-blind placebo- and paroxetine-controlled trials of duloxetine for major depressive disorder. J Clin Psychiatry. 2005 Jun;66(6):686-92. 35. Detke MJ, Wiltse CG, Mallinckrodt CH, McNamara RK, Demitrack MA, Bitter I. Duloxetine in the acute and long-term treatment of major depressive disorder: a placebo- and paroxetine-controlled trial. Eur Neuropsychopharmacol. 2004 Dec;14(6):457-70.

36. Ekselius L, von Knorring L, Eberhard G. A double-blind multicenter trial comparing sertraline and citalopram in patients with major depression treated in general practice. Int Clin Psychopharmacol. 1997 Nov;12(6):323-31. 
37. Fava M, Amsterdam JD, Deltito JA, Salzman C, Schwaller M, Dunner DL. A doubleblind study of paroxetine, fluoxetine, and placebo in outpatients with major depression. Ann Clin Psychiatry. 1998 Dec;10(4):145-50.

38. Feiger A, Kiev A, Shrivastava RK, Wisselink PG, Wilcox CS. Nefazodone versus sertraline in outpatients with major depression: focus on efficacy, tolerability, and effects on sexual function and satisfaction. J Clin Psychiatry. 1996;57 Suppl 2:53-62. 39. Feighner JP, Gardner EA, Johnston JA, Batey SR, Khayrallah MA, Ascher JA, et al. Double-blind comparison of bupropion and fluoxetine in depressed outpatients. J Clin Psychiatry. 1991 Aug;52(8):329-35.

40. Ferguson JM, Shrivastava RK, Stahl SM, Hartford JT, Borian F, Ieni J, et al.

Reemergence of sexual dysfunction in patients with major depressive disorder: doubleblind comparison of nefazodone and sertraline. J Clin Psychiatry. 2001 Jan;62(1):24-9.

41. Gelenberg AJ, Trivedi MH, Rush AJ, Thase ME, Howland R, Klein DN, et al. Randomized, placebo-controlled trial of nefazodone maintenance treatment in preventing recurrence in chronic depression. Biol Psychiatry. 2003 Oct 15;54(8):80617.

42. Gilaberte I, Montejo AL, de la Gandara J, Perez-Sola V, Bernardo M, Massana J, et al. Fluoxetine in the prevention of depressive recurrences: a double-blind study. J Clin Psychopharmacol. 2001 Aug;21(4):417-24.

43. Golden RN, Nemeroff CB, McSorley P, Pitts CD, Dube EM. Efficacy and tolerability of controlled-release and immediate-release paroxetine in the treatment of depression. J Clin Psychiatry. 2002 Jul;63(7):577-84.

44. Guelfi JD, Ansseau M, Timmerman L, Korsgaard S. Mirtazapine versus venlafaxine in hospitalized severely depressed patients with melancholic features. J Clin

Psychopharmacol. 2001 Aug;21(4):425-31.

45. Hicks JA, Argyropoulos SV, Rich AS, Nash JR, Bell CJ, Edwards C, et al. Randomised controlled study of sleep after nefazodone or paroxetine treatment in out-patients with depression. Br J Psychiatry. 2002 Jun;180:528-35.

46. Hochstrasser B, Isaksen PM, Koponen H, Lauritzen L, Mahnert FA, Rouillon F, et al. Prophylactic effect of citalopram in unipolar, recurrent depression: placebo-controlled study of maintenance therapy. Br J Psychiatry. 2001 Apr;178:304-10.

47. Burke WJ, Gergel I, Bose A. Fixed-dose trial of the single isomer SSRI escitalopram in depressed outpatients. J Clin Psychiatry. 2002 Apr;63(4):331-6.

48. Hypericum Depression Trial Study Group. Effect of Hypericum perforatum (St John's wort) in major depressive disorder: a randomized controlled trial. JAMA. 2002 Apr 10;287(14):1807-14.

49. Keller MB, Kocsis JH, Thase ME, Gelenberg AJ, Rush AJ, Koran L, et al. Maintenance phase efficacy of sertraline for chronic depression: a randomized controlled trial. JAMA. 1998 Nov 18;280(19):1665-72.

50. Kiev A, Feiger A. A double-blind comparison of fluvoxamine and paroxetine in the treatment of depressed outpatients. J Clin Psychiatry. 1997 Apr;58(4):146-52.

51. Mehtonen OP, Sogaard J, Roponen P, Behnke K. Randomized, double-blind comparison of venlafaxine and sertraline in outpatients with major depressive disorder. Venlafaxine 631 Study Group. J Clin Psychiatry. 2000 Feb;61(2):95-100.

52. Nemeroff CB, Ninan PT, Ballenger J, Lydiard RB, Feighner J, Patterson WM, et al. Double-blind multicenter comparison of fluvoxamine versus sertraline in the treatment of depressed outpatients. Depression. 1995;3(4):163-9.

53. Perahia DG, Wang F, Mallinckrodt CH, Walker DJ, Detke MJ. Duloxetine in the treatment of major depressive disorder: a placebo- and paroxetine-controlled trial. Eur Psychiatry2006. p. 367-78.

54. Schatzberg A, Roose S. A double-blind, placebo-controlled study of venlafaxine and fluoxetine in geriatric outpatients with major depression. Am J Geriatr Psychiatry2006. p. 361-70.

55. Segraves RT, Kavoussi R, Hughes AR, Batey SR, Johnston JA, Donahue R, et al. Evaluation of sexual functioning in depressed outpatients: a double-blind comparison of sustained-release bupropion and sertraline treatment. J Clin Psychopharmacol. 2000 Apr;20(2):122-8. 
56. Shelton RC, Haman KL, Rapaport MH, Kiev A, Smith WT, Hirschfeld RM, et al. A randomized, double-blind, active-control study of sertraline versus venlafaxine XR in major depressive disorder. J Clin Psychiatry. 2006 Nov;67(11):1674-81.

57. Thase ME. Efficacy and tolerability of once-daily venlafaxine extended release (XR) in outpatients with major depression. The Venlafaxine XR 209 Study Group. J Clin Psychiatry. 1997 Sep;58(9):393-8.

58. Trivedi MH, Pigotti TA, Perera P, Dillingham KE, Carfagno ML, Pitts CD.

Effectiveness of low doses of paroxetine controlled release in the treatment of major depressive disorder. J Clin Psychiatry. 2004 Oct;65(10):1356-64.

59. Ventura D, Armstrong EP, Skrepnek GH, Haim Erder M. Escitalopram versus sertraline in the treatment of major depressive disorder: a randomized clinical trial. Curr Med Res Opin. 2007 Feb;23(2):245-50.

60. Wade A, Gembert K, Florea I. A comparative study of the efficacy of acute and continuation treatment with escitalopram versus duloxetine in patients with major depressive disorder. Curr Med Res Opin. 2007 Jul;23(7):1605-14.

61. Yevtushenko VY, Belous AI, Yevtushenko YG, Gusinin SE, Buzik OJ, Agibalova TV.

Efficacy and tolerability of escitalopram versus citalopram in major depressive disorder: a 6-week, multicenter, prospective, randomized, double-blind, active-controlled study in adult outpatients. Clin Ther2007. p. 2319-32.

62. Nierenberg AA, Greist JH, Mallinckrodt CH, Prakash A, Sambunaris A, Tollefson GD, et al. Duloxetine versus escitalopram and placebo in the treatment of patients with major depressive disorder: onset of antidepressant action, a non-inferiority study. Curr Med Res Opin. 2007 Feb;23(2):401-16.

63. Baldwin DS, Cooper JA, Huusom AK, Hindmarch I. A double-blind, randomized, parallel-group, flexible-dose study to evaluate the tolerability, efficacy and effects of treatment discontinuation with escitalopram and paroxetine in patients with major depressive disorder. Int Clin Psychopharmacol2006. p. 159-69.

64. Baldwin DS, Loft H, Dragheim M. A randomised, double-blind, placebo controlled, duloxetine-referenced, fixed-dose study of three dosages of Lu AA21004 in acute treatment of major depressive disorder (MDD). Eur Neuropsychopharmacol.

2012;22(7):482-91.

65. Bennie EH, Mullin JM, Martindale JJ. A double-blind multicenter trial comparing sertraline and fluoxetine in outpatients with major depression. J Clin Psychiatry. 1995 Jun;56(6):229-37.

66. Bielski RJ, Ventura D, Chang CC. A double-blind comparison of escitalopram and venlafaxine extended release in the treatment of major depressive disorder. J Clin Psychiatry. 2004 Sep;65(9):1190-6.

67. Boulenger JP, Huusom AK, Florea I, Baekdal T, Sarchiapone M. A comparative study of the efficacy of long-term treatment with escitalopram and paroxetine in severely depressed patients. Curr Med Res Opin2006. p. 1331-41.

68. Chouinard G, Saxena B, Belanger MC, Ravindran A, Bakish D, Beauclair L, et al. A Canadian multicenter, double-blind study of paroxetine and fluoxetine in major depressive disorder. J Affect Disord. 1999 Jul;54(1-2):39-48.

69. Cunningham LA. Once-daily venlafaxine extended release (XR) and venlafaxine immediate release (IR) in outpatients with major depression. Venlafaxine XR 208 Study Group. Ann Clin Psychiatry. 1997 Sep;9(3):157-64.

70. Dalery J, Honig A. Fluvoxamine versus fluoxetine in major depressive episode: a double-blind randomised comparison. Hum Psychopharmacol. 2003 Jul;18(5):379-84.

71. Detke MJ, Lu Y, Goldstein DJ, Hayes JR, Demitrack MA. Duloxetine, 60 mg once daily, for major depressive disorder: a randomized double-blind placebo-controlled trial. J Clin Psychiatry. 2002 Apr;63(4):308-15.

72. Fava M, Hoog SL, Judge RA, Kopp JB, Nilsson ME, Gonzales JS. Acute efficacy of fluoxetine versus sertraline and paroxetine in major depressive disorder including effects of baseline insomnia. J Clin Psychopharmacol. 2002 Apr;22(2):137-47.

73. Franchini L, Gasperini M, Perez J, Smeraldi E, Zanardi R. A double-blind study of long-term treatment with sertraline or fluvoxamine for prevention of highly recurrent unipolar depression. J Clin Psychiatry. 1997 Mar;58(3):104-7. 
74. Katona C, Hansen T, Olsen CK. A randomized, double-blind, placebo-controlled, duloxetine-referenced, fixed-dose study comparing the efficacy and safety of $\mathrm{Lu}$ AA21004 in elderly patients with major depressive disorder. Int Clin Psychopharmacol. 2012 Jul;27(4):215-23.

75. Lepola UM, Loft H, Reines EH. Escitalopram (10-20 mg/day) is effective and well tolerated in a placebo-controlled study in depression in primary care. Int Clin Psychopharmacol. 2003 Jul;18(4):211-7.

76. Lineberry CG, Johnston JA, Raymond RN, Samara B, Feighner JP, Harto NE, et al. A fixed-dose (300 mg) efficacy study of bupropion and placebo in depressed outpatients. J Clin Psychiatry. 1990 May;51(5):194-9.

77. Montgomery SA, Huusom AK, Bothmer J. A randomised study comparing escitalopram with venlafaxine XR in primary care patients with major depressive disorder. Neuropsychobiology. 2004;50(1):57-64.

78. Moore N, Verdoux H, Fantino B. Prospective, multicentre, randomized, double-blind study of the efficacy of escitalopram versus citalopram in outpatient treatment of major depressive disorder. Int Clin Psychopharmacol. 2005 May;20(3):131-7.

79. Rabkin JG, Wagner GJ, McElhiney MC, Rabkin R, Lin SH. Testosterone versus fluoxetine for depression and fatigue in HIV/AIDS: a placebo-controlled trial. J Clin Psychopharmacol. 2004 Aug;24(4):379-85.

80. Reimherr FW, Chouinard G, Cohn CK, Cole JO, Itil TM, LaPierre YD, et al. Antidepressant efficacy of sertraline: a double-blind, placebo- and amitriptylinecontrolled, multicenter comparison study in outpatients with major depression. J Clin Psychiatry. 1990 Dec;51 Suppl B:18-27.

81. Rush AJ, Armitage R, Gillin JC, Yonkers KA, Winokur A, Moldofsky H, et al. Comparative effects of nefazodone and fluoxetine on sleep in outpatients with major depressive disorder. Biol Psychiatry. 1998 Jul 1;44(1):3-14.

82. Simon JS, Aguiar LM, Kunz NR, Lei D. Extended-release venlafaxine in relapse prevention for patients with major depressive disorder. J Psychiatr Res. 2004 MayJun;38(3):249-57.

83. Wade A, Michael Lemming O, Bang Hedegaard K. Escitalopram $10 \mathrm{mg} /$ day is effective and well tolerated in a placebo-controlled study in depression in primary care. Int Clin Psychopharmacol. 2002 May;17(3):95-102.

84. Meijer WE, Heerdink ER, van Eijk JT, Leufkens HG. Adverse events in users of sertraline: results from an observational study in psychiatric practice in The Netherlands. Pharmacoepidemiology and drug safety. 2002 Dec;11(8):655-62.

85. Duenas H, Brnabic AJ, Lee A, Montejo AL, Prakash S, Casimiro-Querubin ML, et al. Treatment-emergent sexual dysfunction with SSRIs and duloxetine: effectiveness and functional outcomes over a 6-month observational period. International journal of psychiatry in clinical practice. 2011 Nov;15(4):242-54.

86. Mackay FR, Dunn NR, Martin RM, Pearce GL, Freemantle SN, Mann RD. Newer antidepressants: a comparison of tolerability in general practice. Br J Gen Pract. 1999 Nov;49(448):892-6.

87. Clayton AH, Pradko JF, Croft HA, Montano CB, Leadbetter RA, Bolden-Watson C, et al. Prevalence of sexual dysfunction among newer antidepressants. J Clin Psychiatry. 2002 Apr;63(4):357-66.

88. Ferguson JM. The effects of antidepressants on sexual functioning in depressed patients: a review. J Clin Psychiatry. 2001;62 Suppl 3:22-34.

89. Salanti G. Indirect and mixed-treatment comparison, network, or multipletreatments meta-analysis: many names, many benefits, many concerns for the next generation evidence synthesis tool. Research Synthesis Methods. 2012;3(2):80-97. 90. Bahrick A, Harris M. Sexual Side Effects of Antidepressant Medications: An Informed Consent Accountability Gap. J Contemp Psychother. 2009;39(2):135-43. 
Sexual Dysfunction associated with Second-Generation Antidepressants in Patients with Major Depressive Disorder - Results from a Systematic Review with Network Meta-Analysis. Drug Safety; U. Reichenpfader, G. Gartlehner, L.C. Morgan, A. Greenblatt, B. Nussbaumer, R. A. Hansen, M. Van Noord, L. Lux, and B. N. Gaynes. Corresponding author: U. Reichenpfader, Department for Evidence-based Medicine and Clinical Epidemiology, Krems, Austria; Department of Medical and Health Sciences - Division of Community Medicine, Linköping University, Sweden; ureichenpfader@hotmail.com

\section{Electronic Supplementary Material 1:}

\section{Table S1. Characteristics of Included Studies}

\begin{tabular}{|c|c|c|c|c|c|c|}
\hline $\begin{array}{c}\text { Study } \\
\text { Characteristics }\end{array}$ & $\begin{array}{l}\text { Research Objective } \\
\text { Study Design }\end{array}$ & $\begin{array}{l}\text { Inclusion/Exclusion } \\
\text { Outcome Measures }\end{array}$ & $\begin{array}{c}\text { Population } \\
\text { Characteristics }\end{array}$ & $\begin{array}{l}\text { Sexual Dysfunction } \\
\text { (SD) Outcomes/ } \\
\text { Adverse Events }\end{array}$ & $\begin{array}{l}\text { Method used to } \\
\text { determine SD }\end{array}$ & $\begin{array}{c}\text { Analysis and Study } \\
\text { Quality (Risk of } \\
\text { Bias) }\end{array}$ \\
\hline $\begin{array}{l}\text { Author, Year } \\
\text { Alvarez et al, } 2012 \text { [1] } \\
\text { Country and Setting } \\
\text { multinational, } \\
\text { multicenter } \\
\text { Funding } \\
\text { H. Lundbeck A/S }\end{array}$ & $\begin{array}{l}\text { Research objective } \\
\text { To evaluate efficacy, } \\
\text { safety, and tolerability } \\
\text { of Lu AA21004 vs. } \\
\text { placebo using } \\
\text { venlafaxine XR as } \\
\text { active reference in } \\
\text { patients with DSM-IV- } \\
\text { TR major depressive } \\
\text { disorder (MDD) } \\
\text { Drugs, Doses, and } \\
\text { Range } \\
\text { D1: PBO } \\
\text { D2: VENLA XR (225 } \\
\quad \text { mg } 1 \text { x daily) } \\
\text { Fixed dose } \\
\text { Yes } \\
\text { Flexible dose } \\
\text { No } \\
\text { Study design } \\
\text { phase II RCT, double } \\
\text { blinded } \\
\text { Duration } \\
6 \text { weeks } \\
\text { Type of depression } \\
\text { MDD } \\
\text { Intervention } \\
\text { D1: PBO } \\
\text { D2: Venlafaxine XR }\end{array}$ & $\begin{array}{l}\text { Inclusion criteria: } \\
\text { - Adults (age range): } \\
\text { 18-65 years } \\
\text { - Diagnosed with } \\
\text { MDD according to } \\
\text { DSM-III or -IV: } \\
\text { Current epidsode } \\
\text { of MDD with at } \\
\text { least } 3 \text { months' } \\
\text { duration } \\
\text { - MADRS: total } \\
\text { score } \geq 30 \text { at } \\
\text { baseline visit } \\
\text { Exclusion criteria: } \\
\text { - Concomitant } \\
\text { psychotheraputic } \\
\text { or psychotropic } \\
\text { medications: } \\
\text { current or past } \\
\text { history of manic or } \\
\text { hypomanic } \\
\text { episode, } \\
\text { schizophrenia or } \\
\text { any other psychotic } \\
\text { disorder, including } \\
\text { major depression } \\
\text { with psychotic } \\
\text { features, mental } \\
\text { retardation, organic } \\
\text { mental disorders, } \\
\text { or mental disorders } \\
\text { due to a general } \\
\text { medical condition } \\
\text { - Patients at serious } \\
\text { risk of suicide, } \\
\text { based on the }\end{array}$ & $\begin{array}{l}\text { Groups similar at } \\
\text { baseline } \\
\text { Yes } \\
\mathbf{n =} \\
\text { D1: } 105 \\
\text { D2: } 114 \\
\text { Overall: } 219 \\
\text { Mean age, years } \\
\text { D1: } 42.0 \\
\text { D2: } 45.0 \\
\\
\text { Sex, \% female } \\
\text { D1: } 65.7 \\
\text { D2: } 54.9 \\
\text { Race, \% white } \\
\text { D1: } 93.3 \\
\text { D2: } 92.0 \\
\text { Overall: NR } \\
\text { Baseline MADRS } \\
\text { D1: } 33.9 \\
\text { D2: } 34.2 \\
\text { Overall: NR } \\
\text { Baseline HAMD-24 } \\
\text { D1: } 29.7 \\
\text { D2: } 29.4 \\
\text { Overall: NR }\end{array}$ & $\begin{array}{l}\text { D1: } 2 / 105(1.9 \%) \\
\text { D2: } n=3 \text { of women } \\
\text { reported AE related } \\
\text { to SD ( } 3 / 62,4.8 \% \text { of } \\
\text { women); } n=11 \text { of men } \\
\text { reported } A E \text { related } \\
\text { to SD }(11 / 51,21.6 \% \\
\text { of men); overall \% } \\
\text { with SD } 14 / 114 \\
\text { (12.3\%); } \# \text { AE } \\
\text { reported: anorgasmia } \\
7 / 51(13.7 \% \text { of men); } \\
\text { ejaculation delayed } \\
4 / 51 \text { ( } 7.8 \% \text { of men); } \\
\text { erectile dysfunction } \\
4 / 51 \text { ( } 7.8 \% \text { of men); } 2 \\
\text { withdrawals due to } \\
\text { SD ( } 1 \text { anorgasmia, } 1 \\
\text { delayed ejaculation); }\end{array}$ & $\begin{array}{l}\text { non-leading question } \\
\text { to ascertain AE; no } \\
\text { targeted questions/ } \\
\text { no specific instrument } \\
\text { used to assess SD }\end{array}$ & $\begin{array}{l}\text { Risk of Bias: } \\
\text { low } \\
\text { Withdrawals due to } \\
\text { adverse events, \% } \\
\text { D1: } 4 \\
\text { D2: } 14 \\
\text { Withdrawals due to } \\
\text { lack of efficacy, \% } \\
\text { NR }\end{array}$ \\
\hline
\end{tabular}


Sexual Dysfunction associated with Second-Generation Antidepressants in Patients with Major Depressive Disorder - Results from a Systematic Review with Network Meta-Analysis. Drug Safety; U. Reichenpfader, G. Gartlehner, L.C. Morgan, A. Greenblatt, B. Nussbaumer, R. A. Hansen, M. Van Noord, L. Lux, and B. N. Gaynes. Corresponding author: U. Reichenpfader, Department for Evidence-based Medicine and Clinical Epidemiology, Krems, Austria; Department of Medical and Health Sciences - Division of Community Medicine, Linköping University, Sweden; ureichenpfader@hotmail.com

\begin{tabular}{|c|c|c|c|c|c|c|}
\hline $\begin{array}{c}\text { Study } \\
\text { Characteristics }\end{array}$ & $\begin{array}{l}\text { Research Objective } \\
\text { Study Design }\end{array}$ & $\begin{array}{l}\text { Inclusion/Exclusion } \\
\text { Outcome Measures }\end{array}$ & $\begin{array}{c}\text { Population } \\
\text { Characteristics }\end{array}$ & $\begin{array}{l}\text { Sexual Dysfunction } \\
\text { (SD) Outcomes/ } \\
\text { Adverse Events }\end{array}$ & $\begin{array}{l}\text { Method used to } \\
\text { determine SD }\end{array}$ & $\begin{array}{c}\text { Analysis and Study } \\
\text { Quality (Risk of } \\
\text { Bias) }\end{array}$ \\
\hline & & $\begin{array}{l}\text { investigator's } \\
\text { clinical judgement, } \\
\text { or who had a score } \\
\text { of } 5 \text { on item } 10 \text { of } \\
\text { the MADRS scale } \\
\text { (suicidal thoughts) } \\
\text { - any substance } \\
\text { abuse disorder } \\
\text { within the previous } \\
6 \text { months, } \\
\text { - presence or history } \\
\text { of a clinically } \\
\text { significant } \\
\text { neurological } \\
\text { disorder (including } \\
\text { epilepsy), any } \\
\text { neurodegenerative } \\
\text { disorder, or } \\
\text { - any Axis II disorder } \\
\text { that might } \\
\text { compromise their } \\
\text { participation in the } \\
\text { study Investigation }\end{array}$ & & & & \\
\hline & & $\begin{array}{l}\text { Outcome measures } \\
\text { - MADRS } \\
\text { - Adverse events }\end{array}$ & & & & \\
\hline
\end{tabular}


Sexual Dysfunction associated with Second-Generation Antidepressants in Patients with Major Depressive Disorder - Results from a Systematic Review with Network Meta-Analysis. Drug Safety; U. Reichenpfader, G. Gartlehner, L.C. Morgan, A. Greenblatt, B. Nussbaumer, R. A. Hansen, M. Van Noord, L. Lux, and B. N. Gaynes. Corresponding author: U. Reichenpfader, Department for Evidence-based Medicine and Clinical Epidemiology, Krems, Austria; Department of Medical and Health Sciences - Division of Community Medicine, Linköping University, Sweden; ureichenpfader@hotmail.com

\begin{tabular}{|c|c|c|c|c|c|c|}
\hline $\begin{array}{c}\text { Study } \\
\text { Characteristics }\end{array}$ & $\begin{array}{l}\text { Research Objective } \\
\text { Study Design }\end{array}$ & Inclusion/Exclusion & $\begin{array}{c}\text { Baseline } \\
\text { Characteristics }\end{array}$ & $\begin{array}{c}\text { Sexual Dysfunction } \\
\text { (SD) Outcomes/ } \\
\text { Adverse Events } \\
\end{array}$ & $\begin{array}{l}\text { Method used to } \\
\text { determine SD }\end{array}$ & $\begin{array}{c}\text { Analysis and Study } \\
\text { Quality (Risk of } \\
\text { Bias) }\end{array}$ \\
\hline $\begin{array}{l}\text { Author: } \\
\text { Baldwin et al., } \\
2006 \text { [2] } \\
\text { Country and } \\
\text { Setting } \\
\text { multinational, } \\
\text { multicenter } \\
\text { Funding } \\
\text { H. Lundbeck A/S }\end{array}$ & $\begin{array}{l}\text { Research objective } \\
\text { To evaluate short- and } \\
\text { long-term } \\
\text { antidepressant } \\
\text { tolerability and efficacy } \\
\text { of ESC and PAR. } \\
\text { Drugs, Doses, and } \\
\text { Range } \\
\text { D1: ESC (10-20 mg } 1 \text { x } \\
\quad \text { daily): low-high; } \\
\quad 10-20 \text { mg } \\
\text { D2: PAR (10-60 mg } 1 \text { x } \\
\quad \text { daily): medium; } 20- \\
\quad 40 \text { mg } \\
\text { Fixed dose } \\
\text { No } \\
\text { Flexible dose } \\
\text { Yes } \\
\text { Dosages equivalent } \\
\text { Yes } \\
\text { Study design } \\
\text { RCT } \\
\text { Duration } \\
8 \text { weeks (includes both } \\
\text { acute and } \\
\text { maintainence periods) } \\
\text { Type of depression } \\
\text { MDD } \\
\text { Intervention } \\
\text { D1: PAR } \\
\text { D2: ESC }\end{array}$ & $\begin{array}{l}\text { Inclusion criteria: } \\
\text { - Adults (age range): } \\
18 \text { years old and } \\
\text { over } \\
\text { - Diagnosed with } \\
\text { MDD according to } \\
\text { DSM-III or -IV: } \\
\text { Current epidsode of } \\
\text { MDD } \\
\text { - MADRS: } 22 \text { or } \\
\text { greater and } 40 \text { or } \\
\text { less } \\
\text { Exclusion criteria: } \\
\text { - Concomitant } \\
\text { psychotheraputic or } \\
\text { psychotropic } \\
\text { medications: } \\
\text { includes tryptophan, } \\
\text { benzodiazepines, } \\
\text { antipsychotics, } \\
\text { psychoactive herbal } \\
\text { remedies, MAOls, } \\
\text { prophylactic } \\
\text { treatment dopamine } \\
\text { antagonists } \\
\text { - Schizophrenia, } \\
\text { psychotic disorders, } \\
\text { mania or } \\
\text { hypomania, eating } \\
\text { disorders, } \\
\text { obsessive- } \\
\text { compulsive } \\
\text { disorder, bipolar } \\
\text { disorder } \\
\text { - Investigational drug } \\
\text { use within last } 3 \\
\text { months } \\
\text { - MADRS item } 10 \\
\text { score of } 5 \text { or greater }\end{array}$ & $\begin{array}{l}\text { Groups similar at } \\
\text { baseline } \\
\text { Yes } \\
\text { n= } \\
\text { D1: } 159 \\
\text { D2: } 166 \\
\text { Overall: } 325 \\
\text { Mean age, years } \\
\text { D1: } 45.1 \\
\text { D2: } 44.9 \\
\text { Overall: } 45 \\
\text { Sex, \% female } \\
\text { D1: } 74.7 \\
\text { D2: } 72.7 \\
\text { Overall: } 75.0 \\
\text { Race, \% white } \\
\text { D1: } 99.4 \\
\text { D2: } 98.8 \\
\text { Overall: NR } \\
\text { Baseline HAM-A } \\
\text { NR } \\
\text { Overall: NR } \\
\text { Insomnia, \% } \\
\text { NR } \\
\text { Overall: NR } \\
\text { Concomitant } \\
\text { anergia, \% } \\
\text { NR } \\
\text { Overall: NR } \\
\text { Experienced prior } \\
\text { depressive episodes, } \\
\% \\
\text { NR } \\
\text { Overall: NR } \\
\end{array}$ & $\begin{array}{l}\text { ASEX scores, week } \\
27 \% \text { : } \\
\text { D1: } 57.7 \text { (proportion of } \\
\text { patients with sexual } \\
\text { dysfunction (LOCF } \\
\text { analysis) declined } \\
\text { from baseline ( } 67.3 \%) \\
\text { during both acute } \\
\text { ( } 61.2 \% \text { at week } 8 \text { ) and } \\
\text { maintenance } \\
\text { treatment) } \\
\text { D2: } 57.0 \text { (proportion of } \\
\text { patients with sexual } \\
\text { dysfunction (LOCF } \\
\text { analysis) declined } \\
\text { from baseline (69.9\%) } \\
\text { during both acute } \\
\text { (64.1\% at week } 8 \text { ) and } \\
\text { maintenance } \\
\text { treatment }\end{array}$ & ASEX & $\begin{array}{l}\text { Attrition } \\
\text { Overall attrition, \%: } \\
28 \\
\text { Attrition rate, \%: } \\
\text { D1: } 34 \\
\text { D2: } 21 \\
\text { Study Quality: } \\
\text { Fair }\end{array}$ \\
\hline
\end{tabular}


Sexual Dysfunction associated with Second-Generation Antidepressants in Patients with Major Depressive Disorder - Results from a Systematic Review with Network Meta-Analysis. Drug Safety; U. Reichenpfader, G. Gartlehner, L.C. Morgan, A. Greenblatt, B. Nussbaumer, R. A. Hansen, M. Van Noord, L. Lux, and B. N. Gaynes. Corresponding author: U. Reichenpfader, Department for Evidence-based Medicine and Clinical Epidemiology, Krems, Austria; Department of Medical and Health Sciences - Division of Community Medicine, Linköping University, Sweden; ureichenpfader@hotmail.com

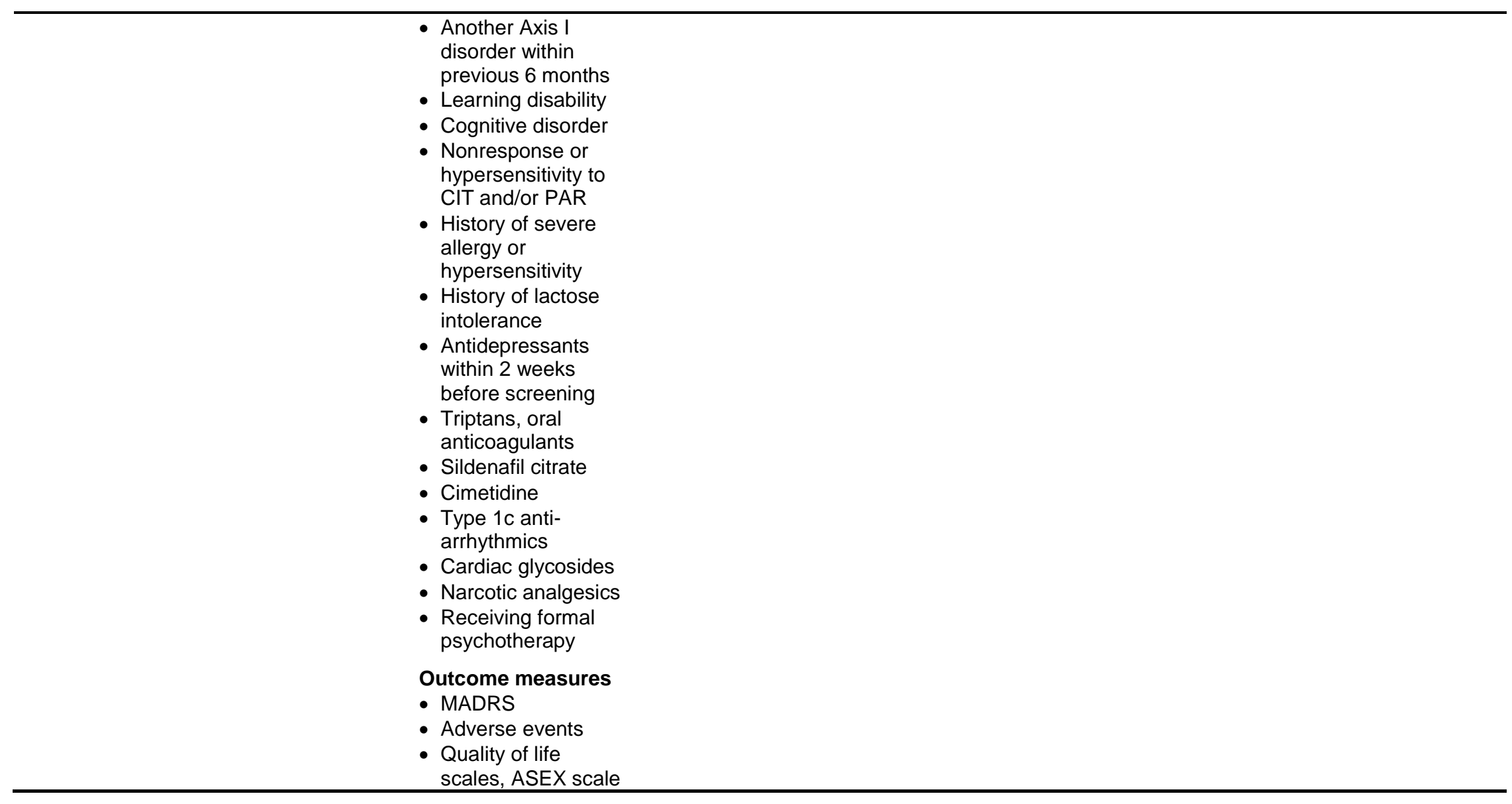


Sexual Dysfunction associated with Second-Generation Antidepressants in Patients with Major Depressive Disorder - Results from a Systematic Review with Network Meta-Analysis. Drug Safety; U. Reichenpfader, G. Gartlehner, L.C. Morgan, A. Greenblatt, B. Nussbaumer, R. A. Hansen, M. Van Noord, L. Lux, and B. N. Gaynes. Corresponding author: U. Reichenpfader, Department for Evidence-based Medicine and Clinical Epidemiology, Krems, Austria; Department of Medical and Health Sciences - Division of Community Medicine,

Linköping University, Sweden; ureichenpfader@hotmail.com

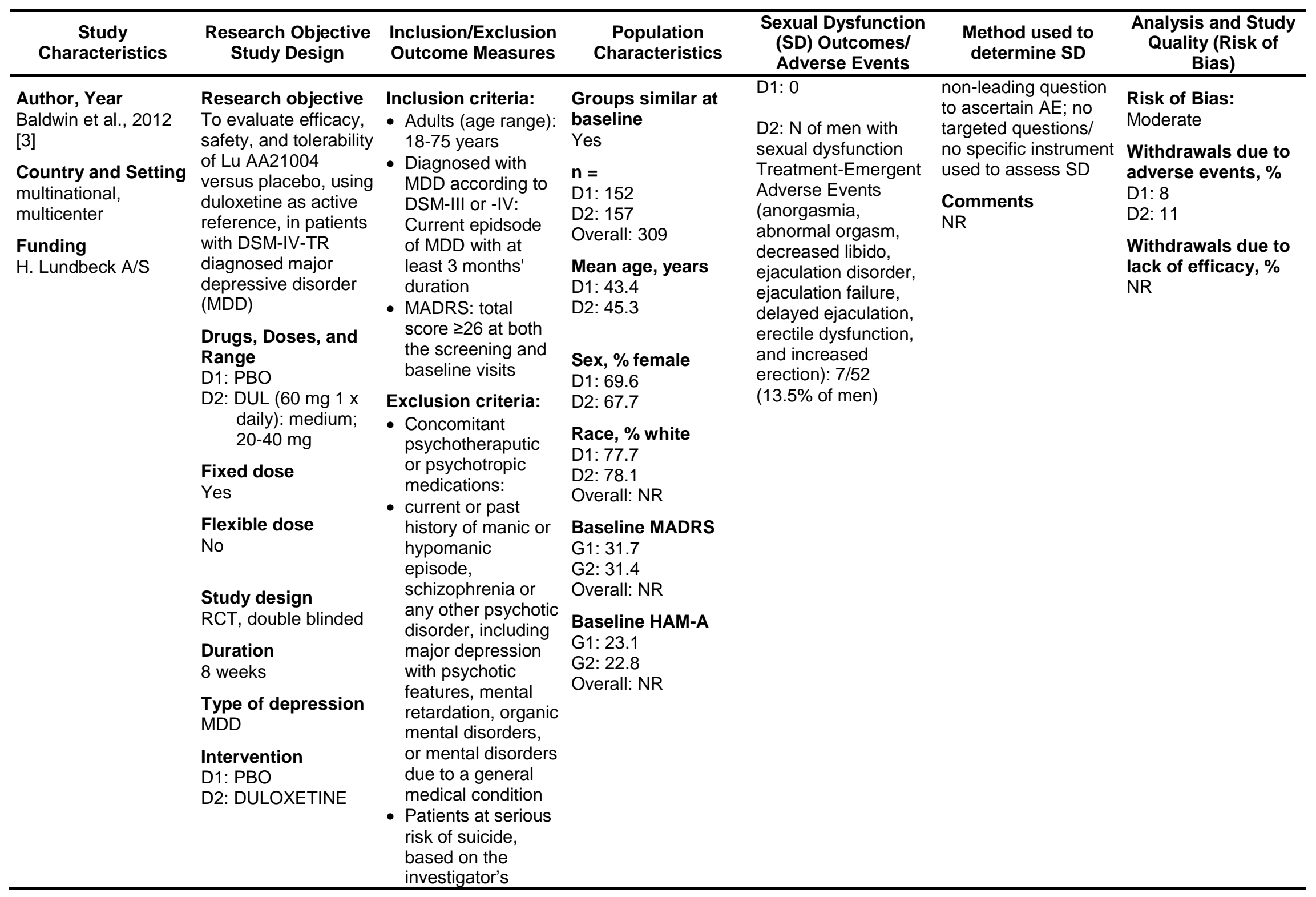


Sexual Dysfunction associated with Second-Generation Antidepressants in Patients with Major Depressive Disorder - Results from a Systematic Review with Network Meta-Analysis. Drug Safety; U. Reichenpfader, G. Gartlehner, L.C. Morgan, A. Greenblatt, B. Nussbaumer, R. A. Hansen, M. Van Noord, L. Lux, and B. N. Gaynes. Corresponding author: U. Reichenpfader, Department for Evidence-based Medicine and Clinical Epidemiology, Krems, Austria; Department of Medical and Health Sciences - Division of Community Medicine, Linköping University, Sweden; ureichenpfader@hotmail.com

\begin{tabular}{|c|c|c|c|c|c|c|}
\hline $\begin{array}{c}\text { Study } \\
\text { Characteristics }\end{array}$ & $\begin{array}{l}\text { Research Objective } \\
\text { Study Design }\end{array}$ & $\begin{array}{l}\text { Inclusion/Exclusion } \\
\text { Outcome Measures }\end{array}$ & $\begin{array}{c}\text { Population } \\
\text { Characteristics }\end{array}$ & $\begin{array}{l}\text { Sexual Dysfunction } \\
\text { (SD) Outcomes/ } \\
\text { Adverse Events }\end{array}$ & $\begin{array}{l}\text { Method used to } \\
\text { determine SD }\end{array}$ & $\begin{array}{c}\text { Analysis and Study } \\
\text { Quality (Risk of } \\
\text { Bias) }\end{array}$ \\
\hline & & $\begin{array}{l}\text { clinical judgement, } \\
\text { or who had a score } \\
\text { of } 5 \text { on item } 10 \text { of } \\
\text { the MADRS scale } \\
\text { (suicidal thoughts) } \\
\text { - any substance } \\
\text { abuse disorder } \\
\text { within the previous } \\
6 \text { months, } \\
\text { - presence or history } \\
\text { of a clinically } \\
\text { significant } \\
\text { neurological } \\
\text { disorder (including } \\
\text { epilepsy), any } \\
\text { neurodegenerative } \\
\text { disorder, or } \\
\text { - any Axis II disorder } \\
\text { that might } \\
\text { compromise their } \\
\text { participation in the } \\
\text { study Investigation }\end{array}$ & & & & \\
\hline & & $\begin{array}{l}\text { Outcome measures } \\
\text { - MADRS } \\
\text { - Adverse events }\end{array}$ & & & & \\
\hline
\end{tabular}


Sexual Dysfunction associated with Second-Generation Antidepressants in Patients with Major Depressive Disorder - Results from a Systematic Review with Network Meta-Analysis. Drug Safety; U. Reichenpfader, G. Gartlehner, L.C. Morgan, A. Greenblatt, B. Nussbaumer, R. A. Hansen, M. Van Noord, L. Lux, and B. N. Gaynes. Corresponding author: U. Reichenpfader, Department for Evidence-based Medicine and Clinical Epidemiology, Krems, Austria; Department of Medical and Health Sciences - Division of Community Medicine,

Linköping University, Sweden; ureichenpfader@hotmail.com

\begin{tabular}{|c|c|c|c|c|c|c|}
\hline $\begin{array}{c}\text { Study } \\
\text { Characteristics }\end{array}$ & $\begin{array}{l}\text { Research Objective } \\
\text { Study Design }\end{array}$ & Inclusion/Exclusion & $\begin{array}{c}\text { Baseline } \\
\text { Characteristics }\end{array}$ & $\begin{array}{c}\text { Sexual Dysfunction } \\
\text { (SD) Outcomes/ } \\
\text { Adverse Events }\end{array}$ & $\begin{array}{l}\text { Method used to } \\
\text { determine SD }\end{array}$ & $\begin{array}{c}\text { Analysis and Study } \\
\text { Quality (Risk of } \\
\text { Bias) }\end{array}$ \\
\hline $\begin{array}{l}\text { Author: } \\
\text { Behnke et al., } \\
\text { 2003[4] } \\
\text { Country and } \\
\text { setting: } \\
\text { Multinational } \\
\text { Multicenter } \\
\text { Funding: } \\
\text { NV Organon }\end{array}$ & $\begin{array}{l}\text { Research objective: } \\
\text { To compare onset of } \\
\text { antidepressant efficacy } \\
\text { of MIR and SER } \\
\text { Duration of study: } \\
8 \text { wks } \\
\text { Study design: } \\
\text { RCT } \\
\text { Overall study N: } \\
346 \\
\text { Intervention: } \\
\text { D1: MIR: } 30-45 \mathrm{mg} / \mathrm{d} \\
\text { D2: SER: } 50-150 \mathrm{mg} / \mathrm{d}\end{array}$ & $\begin{array}{l}\text { Inclusion criteria: } \\
\text { - Adults } 18 \text { to } 70 \\
\text { - Diagnosed with } \\
\text { MDD according to } \\
\text { DSM-III or -IV } \\
\text { - Minimum HAM-D } \\
\text { score of } 18 \\
\text { Exclusion criteria: } \\
\text { - Pregnant } \\
\text { - Lactating } \\
\text { - Concomitant } \\
\text { psychotheraputic or } \\
\text { psychotropic } \\
\text { medications } \\
\text { - Additional mental } \\
\text { illnesses or organic } \\
\text { mental disorder } \\
\text { - Illicit drug and } \\
\text { alcohol abuse } \\
\text { - Clinically sig } \\
\text { medical disease } \\
\text { - Suicidal tendencies } \\
\text { - Epilepsy } \\
\text { - History of seizure } \\
\text { disorder or anti- } \\
\text { convulsant } \\
\text { treatment } \\
\text { - Current eating } \\
\text { disorders diagnosis } \\
\text { - Previous } \\
\text { postpartum } \\
\text { depression or } \\
\text { anxiety disorder } \\
\text { diagnosis } \\
\text { - }\end{array}$ & $\begin{array}{l}\text { Mean age (yrs): } \\
\text { D1: } 42 \\
\text { D2: } 41 \\
\text { Sex (\% female): } \\
\text { D1: } 55.7 \\
\text { D2: } 61.5 \\
\text { Race (\% white): } \\
\text { NR } \\
\text { Baseline (HAM-A): } \\
\text { NR } \\
\text { Mean HAM-D score } \\
\text { at baseline: } \\
\text { NR }\end{array}$ & $\begin{array}{l}\text { CSFQ data not } \\
\text { shown; } A E \text { : decrease: } \\
\text { D1 } 1.1 \% \\
\text { D2: } 5.9 \% \\
P=0.02 \\
\text { Overall adverse } \\
\text { events: } \\
\text { D1: } 64 \\
\text { D2: } 68\end{array}$ & CSFQ; AE method NR & $\begin{array}{l}\text { Overall attrition } \\
\text { rate: } \\
20.8 \% \\
\text { ITT analysis: } \\
\text { Yes } \\
\text { Study Quality: } \\
\text { Fair }\end{array}$ \\
\hline
\end{tabular}


Sexual Dysfunction associated with Second-Generation Antidepressants in Patients with Major Depressive Disorder - Results from a Systematic Review with Network Meta-Analysis. Drug Safety; U. Reichenpfader, G. Gartlehner, L.C. Morgan, A. Greenblatt, B. Nussbaumer, R. A. Hansen, M. Van Noord, L. Lux, and B. N. Gaynes. Corresponding author: U. Reichenpfader, Department for Evidence-based Medicine and Clinical Epidemiology, Krems, Austria; Department of Medical and Health Sciences - Division of Community Medicine, Linköping University, Sweden; ureichenpfader@hotmail.com

\begin{tabular}{|c|c|c|c|c|c|c|}
\hline $\begin{array}{c}\text { Study } \\
\text { Characteristics }\end{array}$ & $\begin{array}{l}\text { Research Objective } \\
\text { Study Design }\end{array}$ & Inclusion/Exclusion & $\begin{array}{c}\text { Baseline } \\
\text { Characteristics }\end{array}$ & $\begin{array}{c}\text { Sexual Dysfunction } \\
\text { (SD) Outcomes/ } \\
\text { Adverse Events }\end{array}$ & $\begin{array}{l}\text { Method used to } \\
\text { determine SD }\end{array}$ & $\begin{array}{c}\text { Analysis and Study } \\
\text { Quality (Risk of } \\
\text { Bias) }\end{array}$ \\
\hline $\begin{array}{l}\text { Author: } \\
\text { Benkert et al., } \\
\text { 2000[5] }\end{array}$ & \multirow{2}{*}{$\begin{array}{l}\text { Research objective: } \\
\text { Safety and efficacy of } \\
\text { MIR and PAR in } \\
\text { treatment of major } \\
\text { depression }\end{array}$} & \multirow{4}{*}{$\begin{array}{l}\text { Inclusion criteria: } \\
\text { - Adults } 18 \text { to } 70 \\
\text { - Diagnosed with } \\
\text { MDD according to } \\
\text { DSM-III or -IV } \\
\text { - Minimum HAM-D } \\
\text { score of } 18\end{array}$} & $\begin{array}{l}\text { Mean age (yrs): } \\
\text { D1: } 47.2 \\
\text { D2: } 47.3\end{array}$ & $\begin{array}{l}\text { D1: orgasmic } \\
\text { dysfunction } 3.1 \% \\
\text { D2: } 13.5 \%\end{array}$ & $\begin{array}{l}\text { UKU Side Effects } \\
\text { Rating Scale }\end{array}$ & $\begin{array}{l}\text { Overall attrition } \\
\text { rate: } \\
23 \%\end{array}$ \\
\hline \multirow{3}{*}{$\begin{array}{l}\text { Country and } \\
\text { setting: } \\
\text { Germany } \\
\text { Multicenter (50) }\end{array}$} & & & \multirow{2}{*}{$\begin{array}{l}\text { Sex (\% female): } \\
\text { D1: } 63 \\
\text { D2: } 65\end{array}$} & $P=0.048$ & & \multirow{4}{*}{$\begin{array}{l}\text { Overall adverse } \\
\text { events: } \\
\text { D1: } 68.1 \\
\text { D2: } 63.4\end{array}$} \\
\hline & Duration of study: & & & & & \\
\hline & 6 wks & & Race (\% white): & & & \\
\hline \multirow{3}{*}{$\begin{array}{l}\text { Funding: } \\
\text { Organon, GmBH, } \\
\text { Munich, } \\
\text { Germany }\end{array}$} & Study design: & \multirow{3}{*}{$\begin{array}{l}\text { Exclusion criteria: } \\
\text { - Pregnant } \\
\text { - Additional mental } \\
\text { illnesses or organic } \\
\text { mental disorder } \\
\text { - Suicidal tendencies }\end{array}$} & & & & \\
\hline & Overall study N: & & $\begin{array}{l}\text { Baseline (HAM-A): } \\
\text { NR }\end{array}$ & & & $\begin{array}{l}\text { ITT analysis: } \\
\text { Yes }\end{array}$ \\
\hline & $\begin{array}{l}\text { Intervention: } \\
\text { D1: MIR: } 15-45 \mathrm{mg} / \mathrm{d} \\
\text { (32.7) } \\
\text { D2: PAR: } 20-40 \mathrm{mg} / \mathrm{d} \\
\text { (22.9) }\end{array}$ & & $\begin{array}{l}\text { Mean HAM-D score } \\
\text { at baseline: } \\
\text { D1: } 22.4(3.3) \\
\text { D2: } 22.4(3.2)\end{array}$ & & & $\begin{array}{l}\text { Study Quality: } \\
\text { Fair }\end{array}$ \\
\hline
\end{tabular}


Sexual Dysfunction associated with Second-Generation Antidepressants in Patients with Major Depressive Disorder - Results from a Systematic Review with Network Meta-Analysis. Drug Safety; U. Reichenpfader, G. Gartlehner, L.C. Morgan, A. Greenblatt, B. Nussbaumer, R. A. Hansen, M. Van Noord, L. Lux, and B. N. Gaynes. Corresponding author: U. Reichenpfader, Department for Evidence-based Medicine and Clinical Epidemiology, Krems, Austria; Department of Medical and Health Sciences - Division of Community Medicine, Linköping University, Sweden; ureichenpfader@hotmail.com

\begin{tabular}{|c|c|c|c|c|c|c|}
\hline $\begin{array}{c}\text { Study } \\
\text { Characteristics }\end{array}$ & $\begin{array}{l}\text { Research Objective } \\
\text { Study Design }\end{array}$ & Inclusion/Exclusion & $\begin{array}{c}\text { Baseline } \\
\text { Characteristics }\end{array}$ & $\begin{array}{l}\text { Sexual Dysfunction } \\
\text { (SD) Outcomes/ } \\
\text { Adverse Events }\end{array}$ & $\begin{array}{l}\text { Method used to } \\
\text { determine SD }\end{array}$ & $\begin{array}{c}\text { Analysis and Study } \\
\text { Quality (Risk of } \\
\text { Bias) }\end{array}$ \\
\hline $\begin{array}{l}\text { Author: } \\
\text { Bennie et al., } \\
\text { 1995[6] } \\
\text { Country and } \\
\text { setting: } \\
\text { UK } \\
\text { Multicenter (20 } \\
\text { centers) } \\
\text { Funding: } \\
\text { Pfizer, Inc }\end{array}$ & $\begin{array}{l}\text { Research objective: } \\
\text { To compare SER and } \\
\text { FLUOX in outpatients } \\
\text { with depression } \\
\text { Duration of study: } \\
6 \text { wks } \\
\text { Study design: } \\
\text { RCT } \\
\text { Overall study N: } \\
286 \\
\text { Intervention: } \\
\text { D1: SER: } 50-100 \mathrm{mg} / \mathrm{d} \\
\text { D2: FLUOX: } 20-40 \\
\quad \text { mg/d }\end{array}$ & $\begin{array}{l}\text { Inclusion criteria: } \\
\text { - Adults } 18 \text { or older } \\
\text { - Diagnosed with } \\
\text { MDD according to } \\
\text { DSM-III or -IV } \\
\text { - Minimum HAM-D } \\
\text { score of } 18 \\
\text { Exclusion criteria: } \\
\text { - Pregnant } \\
\text { - Lactating } \\
\text { - Additional mental } \\
\text { illnesses or organic } \\
\text { mental disorder } \\
\text { - Illicit drug and } \\
\text { alcohol abuse } \\
\text { - Suicidal tendencies }\end{array}$ & $\begin{array}{l}\text { Mean age (yrs): } \\
\text { D1: } 49.9 \\
\text { D2: } 49.9 \\
\text { Sex (\% female): } \\
\text { D1: } 57.7 \\
\text { D2: } 64.6 \\
\text { Race (\% white): } \\
\text { NR } \\
\text { Baseline (HAM-A): } \\
\text { NR } \\
\text { Mean HAM-D score } \\
\text { at baseline: } \\
\text { D1: } 23.2 \\
\text { D2: } 23.4\end{array}$ & $\begin{array}{l}\text { D1: male SD } \\
\text { (ejaculation delayed, } \\
\text { impotence/erectile } \\
\text { dysfunction, reduced } \\
\text { libido): } 3 / 60 \text { ( } 5 \% \text { of } \\
\text { men) } \\
\text { D2: male SD } \\
\text { (ejaculation delayed, } \\
\text { impotence/erectile } \\
\text { dysfunction, reduced } \\
\text { libido): } 2 / 51 \text { (3.9\% of } \\
\text { men) }\end{array}$ & spontaneously reported & $\begin{array}{l}\text { Overall attrition } \\
\text { rate: } \\
13.3 \% \\
\text { Overall adverse } \\
\text { events: } \\
\text { D1: } 56 \\
\text { D2: } 60 \\
\text { ITT analysis: } \\
\text { Yes } \\
\text { Study Quality: } \\
\text { Fair }\end{array}$ \\
\hline
\end{tabular}


Sexual Dysfunction associated with Second-Generation Antidepressants in Patients with Major Depressive Disorder - Results from a Systematic Review with Network Meta-Analysis. Drug Safety; U. Reichenpfader, G. Gartlehner, L.C. Morgan, A. Greenblatt, B. Nussbaumer, R. A. Hansen, M. Van Noord, L. Lux, and B. N. Gaynes. Corresponding author: U. Reichenpfader, Department for Evidence-based Medicine and Clinical Epidemiology, Krems, Austria; Department of Medical and Health Sciences - Division of Community Medicine, Linköping University, Sweden; ureichenpfader@hotmail.com

\begin{tabular}{|c|c|c|c|c|c|c|}
\hline $\begin{array}{c}\text { Study } \\
\text { Characteristics }\end{array}$ & $\begin{array}{l}\text { Research Objective } \\
\text { Study Design }\end{array}$ & Inclusion/Exclusion & $\begin{array}{c}\text { Baseline } \\
\text { Characteristics }\end{array}$ & $\begin{array}{c}\text { Sexual Dysfunction } \\
\text { (SD) Outcomes/ } \\
\text { Adverse Events }\end{array}$ & $\begin{array}{l}\text { Method used to } \\
\text { determine SD }\end{array}$ & $\begin{array}{c}\text { Analysis and Risk } \\
\text { of Bias }\end{array}$ \\
\hline $\begin{array}{l}\text { Author: } \\
\text { Bielski et al., } \\
\text { 2004[7] }\end{array}$ & \multirow{2}{*}{$\begin{array}{l}\text { Research objective: } \\
\text { To compare ESC and } \\
\text { VEN XR in depressed } \\
\text { outpatients at highest } \\
\text { recommended doses in } \\
\text { United States }\end{array}$} & \multirow{3}{*}{$\begin{array}{l}\text { Inclusion criteria: } \\
\text { - Adults } 18 \text { to } 65 \\
\text { - Diagnosed with } \\
\text { MDD according to } \\
\text { DSM-III or -IV } \\
\text { - HAM-D24 > } 20 \\
\text { - Normal physical } \\
\text { exam, labs, and } \\
\text { ECG (or any } \\
\text { abnormality } \\
\text { insignficant) } \\
\text { - Using contraceptive }\end{array}$} & $\begin{array}{l}\text { Mean age (yrs): } \\
\text { D1: } 37.3 \\
\text { D2: } 37.5\end{array}$ & $\begin{array}{l}\text { D1: ejaculation } \\
\text { disorder } 2 / 30(6.7 \% \text { of } \\
\text { men) }\end{array}$ & NR & $\begin{array}{l}\text { Overall attrition } \\
\text { rate: } \\
30 \%\end{array}$ \\
\hline $\begin{array}{l}\text { Country and } \\
\text { setting: } \\
\text { United States } \\
\text { Outpatient } \\
\text { centers }\end{array}$ & & & $\begin{array}{l}\text { Sex (\% female): } \\
\text { D1: } 69.4 \\
\text { D2: } 47.0 \\
\text { Race (\% white): } \\
\text { D1: } 77.6\end{array}$ & $\begin{array}{l}\text { D2: ejaculation } \\
\text { disorder } 12 / 53(22.6 \% \\
\text { of men) }\end{array}$ & & $\begin{array}{l}\text { Overall adverse } \\
\text { events: } \\
\text { D1: } 68 \\
\text { D2: } 85\end{array}$ \\
\hline $\begin{array}{l}\text { Funding: } \\
\text { Forrest } \\
\text { Laboratories, Inc }\end{array}$ & $\begin{array}{l}\text { Study design: } \\
\text { RCT } \\
\text { Overall study } \mathrm{N} \text { : }\end{array}$ & & $\begin{array}{l}\text { D2: } 73.0 \\
\text { Baseline (HAM-A): } \\
\text { NR }\end{array}$ & & & $\begin{array}{l}\text { ITT analysis: } \\
\text { Yes }\end{array}$ \\
\hline & $\begin{array}{l}198 \\
\text { Intervention: } \\
\text { D1: ESC: 20mg } \\
\text { D2: VEN: XR 225mg }\end{array}$ & $\begin{array}{l}\text { Exclusion criteria: } \\
\text { - Pregnant } \\
\text { - Lactating } \\
\text { - Concomitant } \\
\text { psychotheraputic or } \\
\text { psychotropic } \\
\text { medications } \\
\text { - Additional mental } \\
\text { illnesses or organic } \\
\text { mental } \\
\text { - Illicit drug and } \\
\text { alcohol abuse } \\
\text { - Clinically sig } \\
\text { medical disease } \\
\text { - Suicidal tendencies } \\
\text { - Previous treatment } \\
\text { with VEN or ESC } \\
\text { - Failure to respond } \\
\text { to adequate trials of } \\
2+\text { antidepressants }\end{array}$ & $\begin{array}{l}\text { Mean HAM-D score } \\
\text { at baseline: } \\
\text { D1: } 28.6(4.1) \\
\text { D2: } 27.4(4.5)\end{array}$ & & & $\begin{array}{l}\text { Risk of Bias: } \\
\text { Fair }\end{array}$ \\
\hline
\end{tabular}


Sexual Dysfunction associated with Second-Generation Antidepressants in Patients with Major Depressive Disorder - Results from a Systematic Review with Network Meta-Analysis. Drug Safety; U. Reichenpfader, G. Gartlehner, L.C. Morgan, A. Greenblatt, B. Nussbaumer, R. A. Hansen, M. Van Noord, L. Lux, and B. N. Gaynes. Corresponding author: U. Reichenpfader, Department for Evidence-based Medicine and Clinical Epidemiology, Krems, Austria; Department of Medical and Health Sciences - Division of Community Medicine,

Linköping University, Sweden; ureichenpfader@hotmail.com

\begin{tabular}{|c|c|c|c|c|c|c|}
\hline $\begin{array}{c}\text { Study } \\
\text { Characteristics }\end{array}$ & $\begin{array}{l}\text { Research Objective } \\
\text { Study Design }\end{array}$ & $\begin{array}{l}\text { Inclusion/Exclusion } \\
\text { Outcome Measures }\end{array}$ & $\begin{array}{c}\text { Population } \\
\text { Characteristics }\end{array}$ & $\begin{array}{c}\text { Sexual Dysfunction } \\
\text { (SD) Outcomes/ } \\
\text { Adverse Events } \\
\end{array}$ & $\begin{array}{l}\text { Method used to } \\
\text { determine SD }\end{array}$ & $\begin{array}{c}\text { Analysis and Study } \\
\text { Quality (Risk of } \\
\text { Bias) }\end{array}$ \\
\hline $\begin{array}{l}\text { Author, Year } \\
\text { Boulenger et } \\
\text { al., 2006[8] } \\
\text { Country and } \\
\text { Setting } \\
\text { Multinational; } \\
\text { psychiatric and } \\
\text { primary care } \\
\text { settings } \\
\text { Funding } \\
\text { H. Lundbeck } \\
\text { A/S }\end{array}$ & 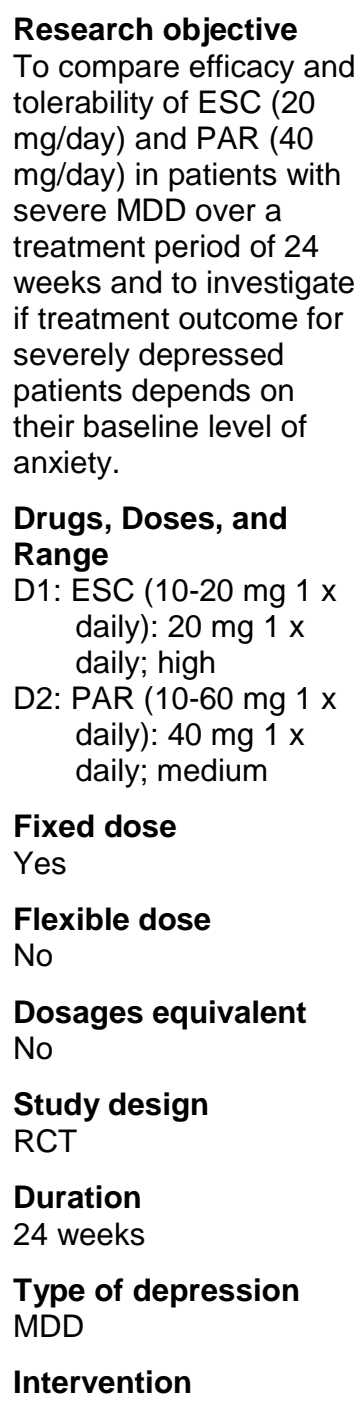 & $\begin{array}{l}\text { Inclusion criteria: } \\
\text { - Adults (age range): } \\
18 \text { to } 75 \text { years } \\
\text { - Diagnosed with } \\
\text { MDD according to } \\
\text { DSM-III or -IV } \\
\text { - MADRS: score } \\
\text { greater than or } \\
\text { equal } 30 \text { at } \\
\text { baseline } \\
\text { - Duration of } \\
\text { depressive episode } \\
\text { had to be more } \\
\text { than } 2 \text { wks, but less } \\
\text { than } 1 \text { yr } \\
\text { Exclusion criteria: } \\
\text { - Pregnant } \\
\text { - Lactating } \\
\text { - Concomitant } \\
\text { psychotheraputic or } \\
\text { psychotropic } \\
\text { medications } \\
\text { - Bipolar disorder, } \\
\text { psychotic disorder } \\
\text { or features, } \\
\text { obsessive- } \\
\text { compulsive } \\
\text { disorder, current } \\
\text { eating disorders } \\
\text { (anorexia nervosa, } \\
\text { bulimia), mental } \\
\text { retardation, any } \\
\text { pervasive } \\
\text { developmental } \\
\text { disorder or } \\
\text { cognitive disorder } \\
\text { - Illicit drug and } \\
\text { alcohol abuse: } \\
\text { Comata }\end{array}$ & $\begin{array}{l}\text { Groups similar at } \\
\text { baseline } \\
\text { Yes } \\
\text { n= } \\
\text { D1: } 232 \\
\text { D2: } 227 \\
\text { (For subgroup } \\
\text { analysis of highly } \\
\text { anxious patients: } \\
\text { n=286) } \\
\text { Mean age, years } \\
\text { D1: } 43.8(12.5) \\
\text { D2: } 44.7 \text { (13.0) } \\
\text { Sex, \% female } \\
\text { D1: } 67 \\
\text { D2: } 70 \\
\text { Race, \% white } \\
\text { D1: } 97.8 \\
\text { D2: } 99.6 \\
\text { Baseline HAM-A } \\
\text { D1: } 23.5 \text { (7.5) } \\
\text { D2: } 23.5 \text { (7.1) } \\
\text { Insomnia, \% } \\
\text { NR } \\
\text { Concomitant } \\
\text { anergia, \% } \\
\text { NR } \\
\text { Experienced prior } \\
\text { depressive } \\
\text { episodes, \% } \\
\text { NR }\end{array}$ & $\begin{array}{l}\text { D1: } 8 \% \text { of men; } \\
\text { erectile dysfunction } \\
\text { (men): } n=4 \text { (of } 153 \\
\text { with } A E ; \text { ( } 5.3 \% \text { of } \\
\text { men); ejaculation } \\
\text { delayed (men), } n=2 \text { ( } \\
2.7 \% \text { of men) } \\
\text { D2: } 14.7 \% \text { of men } \\
\text { (ns); erectile } \\
\text { dysfunction (men): } \\
\text { paroxetine } n=4 \text { (of } \\
162 \text { with } A E ; 5.9 \% \text { of } \\
\text { men); escitalopram: } \\
n=4 \text { (of } 153 \text { with } A E ; \\
\text { (5.3\% of men); } \\
\text { ejaculation delayed } \\
\text { (men): paroxetine } n=6 \\
\text { (of } 162 \text { with } A E ; 8.8 \% \\
\text { of men), escitalopram: } \\
n=2 \text { (of } 153 \text { with } A E ; \\
2.7 \% \text { of men) }\end{array}$ & $\begin{array}{l}\text { spontaneously } \\
\text { reported }\end{array}$ & $\begin{array}{l}\text { Overall adverse } \\
\text { events, \%: } \\
\text { D1: } 7.8 \\
\text { D2: } 15.4 \\
\text { Study Quality: } \\
\text { Fair } \\
\text { Attrition } \\
\text { Overall attrition, \%: } \\
26.3 \% \text { attrition rate } \\
\text { based on number of } \\
\text { patients randomized, } \\
\mathrm{n}=459 . \\
\text { Attrition rate, \%: } \\
\text { D1: } 20,3 \\
\text { D2: } 32,6 \\
\text { Withdrawals due to } \\
\text { adverse events, \% } \\
\text { D1: } 7.8 \\
\text { D2: } 15.4 \\
\text { Withdrawals due to } \\
\text { lack of efficacy, \% } \\
\text { D1: } 4.3 \\
\text { D2: } 6.2 \\
\text { Comments } \\
\text { The calculations were } \\
\text { based on number of } \\
\text { patients randomized } \\
\text { (Overall } n=459 ; E S C \\
20 \text { mg/day } n=232, \\
\text { PAR } 40 \text { mg/day } n= \\
227) . \\
\text { Significantly more } \\
\text { patients ( } P<0.01) \\
\text { withdrew from PAR }\end{array}$ \\
\hline
\end{tabular}


Sexual Dysfunction associated with Second-Generation Antidepressants in Patients with Major Depressive Disorder - Results from a Systematic Review with Network Meta-Analysis. Drug Safety; U. Reichenpfader, G. Gartlehner, L.C. Morgan, A. Greenblatt, B. Nussbaumer, R. A. Hansen, M. Van Noord, L. Lux, and B. N. Gaynes. Corresponding author: U. Reichenpfader, Department for Evidence-based Medicine and Clinical Epidemiology, Krems, Austria; Department of Medical and Health Sciences - Division of Community Medicine, Linköping University, Sweden; ureichenpfader@hotmail.com

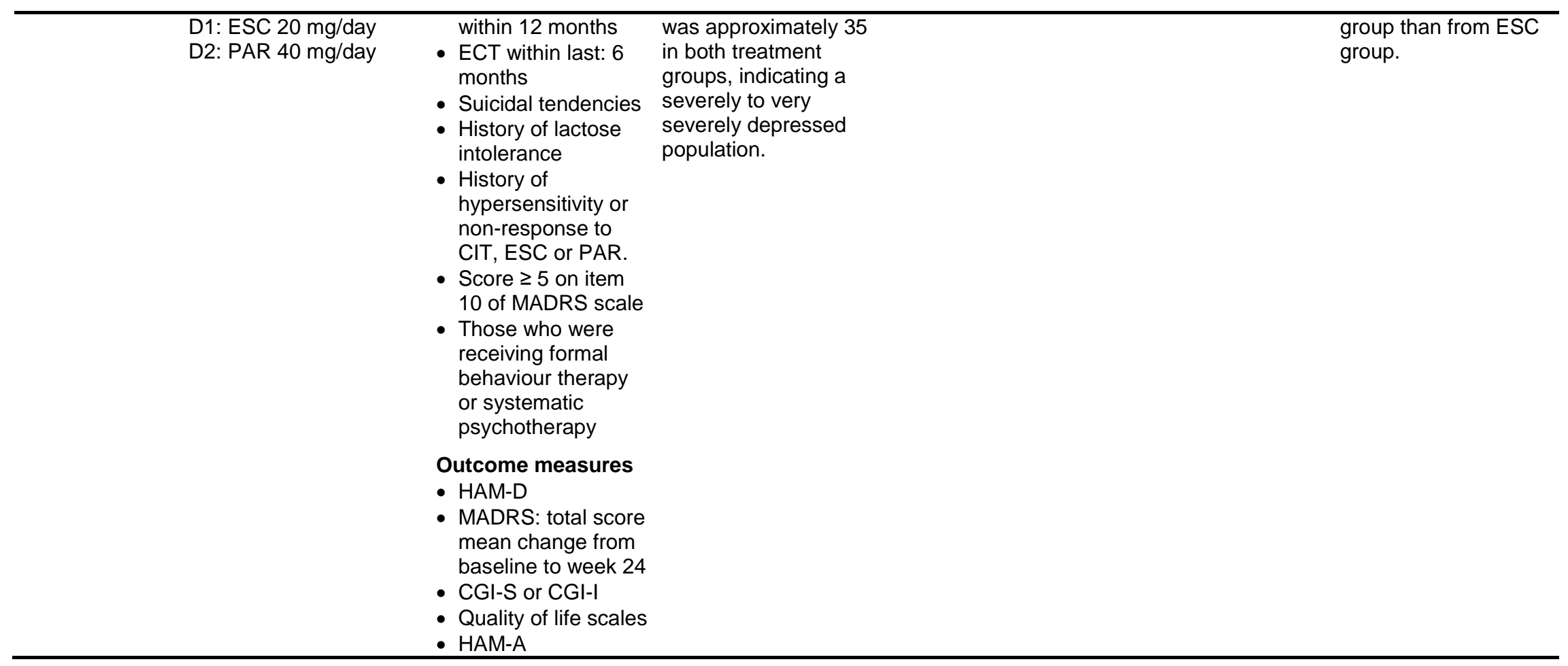


Sexual Dysfunction associated with Second-Generation Antidepressants in Patients with Major Depressive Disorder - Results from a Systematic Review with Network Meta-Analysis. Drug Safety; U. Reichenpfader, G. Gartlehner, L.C. Morgan, A. Greenblatt, B. Nussbaumer, R. A. Hansen, M. Van Noord, L. Lux, and B. N. Gaynes. Corresponding author: U. Reichenpfader, Department for Evidence-based Medicine and Clinical Epidemiology, Krems, Austria; Department of Medical and Health Sciences - Division of Community Medicine, Linköping University, Sweden; ureichenpfader@hotmail.com

\begin{tabular}{|c|c|c|c|c|c|c|}
\hline $\begin{array}{c}\text { Study } \\
\text { Characteristics }\end{array}$ & $\begin{array}{l}\text { Research Objective } \\
\text { Study Design }\end{array}$ & Inclusion/Exclusion & $\begin{array}{c}\text { Baseline } \\
\text { Characteristics }\end{array}$ & $\begin{array}{c}\text { Sexual Dysfunction } \\
\text { (SD) Outcomes/ } \\
\text { Adverse Events } \\
\end{array}$ & $\begin{array}{l}\text { Method used to } \\
\text { determine SD }\end{array}$ & $\begin{array}{c}\text { Analysis and Study } \\
\text { Quality (Risk of } \\
\text { Bias) }\end{array}$ \\
\hline $\begin{array}{l}\text { Author: } \\
\text { Burke et al., } \\
2002 \text { [9] } \\
\text { Country and } \\
\text { setting: } \\
\text { United States } \\
\text { Multicenter (35 } \\
\text { centers) } \\
\text { Funding: } \\
\text { Forest } \\
\text { Laboratories }\end{array}$ & $\begin{array}{l}\text { Research objective: } \\
\text { To evaluate efficacy } \\
\text { and tolerability of ESC } \\
\text { in treatment of MDD } \\
\text { Duration of study: } \\
8 \text { wks } \\
\text { Study design: } \\
\text { RCT } \\
\text { Overall study N: } \\
369 \\
\text { Intervention: } \\
\text { D1: PBO } \\
\text { D2: ESC } 10 \mathrm{mg} / \mathrm{d} \\
\text { D3: ESC } 20 \mathrm{mg} / \mathrm{d} \\
\text { D4: CIT } 40 \mathrm{mg} / \mathrm{d}\end{array}$ & $\begin{array}{l}\text { Inclusion criteria: } \\
\text { - Adults } 18 \text { to } 65 \\
\text { - Diagnosed with } \\
\text { MDD according to } \\
\text { DSM-III or -IV } \\
\text { - Minimum HAM-D } \\
\text { score of at least } 2 \\
\text { on item } 1 \\
\text { (depressed mood) } \\
\text { - Depressive episode } \\
\geq 4 \text { wks } \\
\text { - MADRS } 22 \\
\text { Exclusion criteria: } \\
\text { - Pregnant } \\
\text { - Lactating } \\
\text { - Concomitant } \\
\text { psychotheraputic or } \\
\text { psychotropic } \\
\text { medications } \\
\text { - Illicit drug and } \\
\text { alcohol abuse } \\
\text { - Suicidal tendencies } \\
\text { - Any DSM-IV Axis I } \\
\text { disorder other than } \\
\text { MDD } \\
\text { - Score at least } 5 \text { on } \\
\text { item } 10 \text { of MADRS }\end{array}$ & $\begin{array}{l}\text { Mean age (yrs): } \\
\text { D1: } 40.1 \\
\text { D2: } 40.7 \\
\text { D3: } 39.6 \\
\text { D4: } 40.0 \\
\text { Sex (\% female): } \\
\text { D1: } 60 \\
\text { D2: } 70 \\
\text { D3: } 68 \\
\text { D4: } 62 \\
\text { Race (\% white): } \\
\text { NR } \\
\text { Baseline (HAM-A): } \\
\text { NR } \\
\text { Mean HAM-D score } \\
\text { at baseline: } \\
\text { D1: } 25.8(5.9) \\
\text { D2: } 24.3(6.2) \\
\text { D3: } 25.8(5.7) \\
\text { D4: } 25.9(5.9)\end{array}$ & $\begin{array}{l}\text { Sexual dysfunction } \\
\text { (\%): } \\
\text { D1: } 0 \\
\text { D2: } 9 \\
\text { D3: } 12 \\
\text { D4: } 4\end{array}$ & NR & $\begin{array}{l}\text { Overall attrition } \\
\text { rate: } \\
24 \% \\
\text { Overall adverse } \\
\text { events: } \\
\text { D1: } 70.5 \\
\text { D2: } 79 \\
\text { D3: } 85.6 \\
\text { D4: } 86.4\end{array}$ \\
\hline
\end{tabular}


Sexual Dysfunction associated with Second-Generation Antidepressants in Patients with Major Depressive Disorder - Results from a Systematic Review with Network Meta-Analysis. Drug Safety; U. Reichenpfader, G. Gartlehner, L.C. Morgan, A. Greenblatt, B. Nussbaumer, R. A. Hansen, M. Van Noord, L. Lux, and B. N. Gaynes. Corresponding author: U. Reichenpfader, Department for Evidence-based Medicine and Clinical Epidemiology, Krems, Austria; Department of Medical and Health Sciences - Division of Community Medicine, Linköping University, Sweden; ureichenpfader@hotmail.com

\begin{tabular}{|c|c|c|c|c|c|c|}
\hline $\begin{array}{c}\text { Study } \\
\text { Characteristics }\end{array}$ & $\begin{array}{l}\text { Research Objective } \\
\text { Study Design }\end{array}$ & Inclusion/Exclusion & $\begin{array}{c}\text { Baseline } \\
\text { Characteristics }\end{array}$ & $\begin{array}{l}\text { Sexual Dysfunction } \\
\text { (SD) Outcomes/ } \\
\text { Adverse Events }\end{array}$ & $\begin{array}{l}\text { Method used to } \\
\text { determine SD }\end{array}$ & $\begin{array}{c}\text { Analysis and Study } \\
\text { Quality (Risk of } \\
\text { Bias) }\end{array}$ \\
\hline $\begin{array}{l}\text { Author: } \\
\text { Chouinard et al., } \\
\text { 1999[10] } \\
\text { Country and } \\
\text { setting: } \\
\text { Canada } \\
\text { Multicenter (8) } \\
\text { Funding: } \\
\text { SmithKline, } \\
\text { Beecham }\end{array}$ & $\begin{array}{l}\text { Research objective: } \\
\text { To evaluate } \\
\text { antidepressant and } \\
\text { anxiolytic efficacy of } \\
\text { PAR and FLUOX in } \\
\text { patients with major } \\
\text { depression } \\
\text { Duration of study: } \\
12 \text { wks } \\
\text { Study design: } \\
\text { RCT } \\
\text { Overall study N: } \\
\text { 203 } \\
\text { Intervention: } \\
\text { D1: PAR: } 20-50 \text { mg/d } \\
\text { D2: FLUOX: } 20-80 \\
\quad \text { mg/d }\end{array}$ & $\begin{array}{l}\text { Inclusion criteria: } \\
\text { - Diagnosed with } \\
\text { MDD according to } \\
\text { DSM-III or -IV } \\
\text { - Minimum HAM-D } \\
\text { score of } 20 \text { and } \\
\text { score of } 2 \text { on HAM- } \\
\text { D item } 1 \\
\text { - Depression } \\
\text { symptoms for at } \\
\text { least } 1 \text { mo } \\
\text { Exclusion criteria: } \\
\text { - Pregnant or } \\
\text { lactating } \\
\text { - Concomitant } \\
\text { psychotheraputic or } \\
\text { psychotropic } \\
\text { medications } \\
\text { - Additional mental } \\
\text { illnesses or organic } \\
\text { mental disorder } \\
\text { - Illicit drug and } \\
\text { alcohol abuse } \\
\text { - Clinically sig } \\
\text { medical disease } \\
\text { - ECT within last } 2 \\
\text { mos } \\
\text { - Suicidal tendencies }\end{array}$ & $\begin{array}{l}\text { Mean age (yrs): } \\
\text { D1: } 40.6 \\
\text { D2: } 41.2 \\
\text { Sex (\% female): } \\
\text { D1: } 63.7 \\
\text { D2: } 59.4 \\
\text { Race (\% white): } \\
\text { D1: } 95.1 \\
\text { D2: } 98.0 \\
\text { Baseline (HAM-A): } \\
\text { NR } \\
\text { Mean HAM-D score } \\
\text { at baseline: } \\
\text { D1: } 25.91(0.46) \\
\text { D2: } 25.45(0.46)\end{array}$ & $\begin{array}{l}\text { Sexual dysfunction: } \\
\text { D1: abnormal } \\
\text { ejaculation: } 24.3 \% \text { of } \\
\text { men, } \\
\text { impotence/erectile } \\
\text { dysfunction: } 10.8 \% \\
\text { D2: abnormal } \\
\text { ejaculation: } 12.2 \% \text { of } \\
\text { men, } \\
\text { impotence/erectile } \\
\text { dysfunction: } 7.3 \% \text { of } \\
\text { men }\end{array}$ & NR & $\begin{array}{l}\text { Overall attrition } \\
\text { rate: } \\
36 \% \\
\text { ITT analysis: } \\
\text { Yes } \\
\text { Study Quality: } \\
\text { Fair }\end{array}$ \\
\hline
\end{tabular}


Sexual Dysfunction associated with Second-Generation Antidepressants in Patients with Major Depressive Disorder - Results from a Systematic Review with Network Meta-Analysis. Drug Safety; U. Reichenpfader, G. Gartlehner, L.C. Morgan, A. Greenblatt, B. Nussbaumer, R. A. Hansen, M. Van Noord, L. Lux, and B. N. Gaynes. Corresponding author: U. Reichenpfader, Department for Evidence-based Medicine and Clinical Epidemiology, Krems, Austria; Department of Medical and Health Sciences - Division of Community Medicine, Linköping University, Sweden; ureichenpfader@hotmail.com

\begin{tabular}{|c|c|c|c|c|c|c|}
\hline $\begin{array}{c}\text { Study } \\
\text { Characteristics }\end{array}$ & $\begin{array}{l}\text { Research Objective } \\
\text { Study Design }\end{array}$ & Inclusion/Exclusion & $\begin{array}{c}\text { Baseline } \\
\text { Characteristics }\end{array}$ & $\begin{array}{c}\text { Sexual Dysfunction } \\
\text { (SD) Outcomes/ } \\
\text { Adverse Events } \\
\end{array}$ & $\begin{array}{l}\text { Method used to } \\
\text { determine SD }\end{array}$ & $\begin{array}{c}\text { Analysis and Study } \\
\text { Quality (Risk of } \\
\text { Bias) }\end{array}$ \\
\hline $\begin{array}{l}\text { Author: } \\
\text { Clayton et al., } \\
2002 \text { [11] } \\
\text { Country and } \\
\text { setting: } \\
\text { USA, } \\
\text { Multicenter, } \\
\text { primary care } \\
\text { Funding: } \\
\text { Glaxo Wellcome } \\
\text { Inc., }\end{array}$ & $\begin{array}{l}\text { Research objective: } \\
\text { To compare sexual } \\
\text { functioning associated } \\
\text { with newer } \\
\text { antidepressants using a } \\
\text { validated scale in } \\
\text { patients with MDD } \\
\text { Duration of study: } \\
\text { Study design: } \\
\text { observational/ cross- } \\
\text { sectional } \\
\text { Overall study N: } \\
\text { 6000+ } \\
\text { Intervention: } \\
\text { D1: Bupropion IR, } \\
\text { 255mg/d (mean } \\
\text { dose) } \\
\text { D2: Bupropion SR, } \\
\text { 273.7mg/d (mean } \\
\text { dose) } \\
\text { D3: Citalopram, } \\
24.9 m g / d \text { (mean } \\
\text { dose) } \\
\text { D4: Fluoxetine, } \\
25.5 \mathrm{mg} / \mathrm{d} \text { (mean } \\
\text { dose) } \\
\text { D5: Paroxetine, } \\
23.3 \mathrm{mg} / \mathrm{d} \text { (mean } \\
\text { dose) } \\
\text { D6: Sertraline, } \\
81.4 \mathrm{mg} / \mathrm{d} \text { (mean } \\
\text { dose) } \\
\text { D7: Venlafaxine XR, } \\
\text { 114.9mg/d (mean } \\
\text { dose) }\end{array}$ & $\begin{array}{l}\text { Inclusion criteria: } \\
\text { - Aged at least } 18 \\
\text { years } \\
\text { - receiving } \\
\text { monotherapy for } \\
\text { depression (using } \\
\text { one of the newer } \\
\text { antidepressants: } \\
\text { Bupropion IR/SR, } \\
\text { Citalopram, } \\
\text { Fluoxetine, } \\
\text { Mirtazapine, } \\
\text { Nefazodone, } \\
\text { Paroxetine, } \\
\text { Sertraline, } \\
\text { Venlafaxine or } \\
\text { Venlafaxine XR } \\
\text { - sexually active at } \\
\text { some point during } \\
\text { the prior } 12 \text { months } \\
\text { Exclusion criteria: } \\
\text { - Concomitant } \\
\text { psychotheraputic or } \\
\text { psychotropic } \\
\text { medications }\end{array}$ & $\begin{array}{l}\text { Target population: } \\
\text { Mean age (yrs): } \\
\text { overall } 30.2 \\
\text { Sex (\% female): } \\
\text { overall: } 77.2 \\
\text { Race (\% white): } \\
\text { overall: } 93.1 \\
\text { Baseline (HAMD-24): } \\
\text { NR } \\
\text { Mean MADRS score } \\
\text { at baseline: } \\
\text { NR }\end{array}$ & $\begin{array}{l}\text { Prevalence of SD } \\
\text { (CSFQ-14), \% } \\
\text { D1: NR } \\
\text { D2: } 7 \\
\text { D3: } 30 \\
\text { D4: } 23 \text { (only graph } \\
\text { shown, data not } \\
\text { reported) } \\
\text { D5: } 25 \text { (only graph } \\
\text { shown, data not } \\
\text { reported) } \\
\text { D6: } 25 \text { (only graph } \\
\text { shown, data not } \\
\text { reported) } \\
\text { D7: } 30\end{array}$ & $\begin{array}{l}\text { CSFQ-14, gender- } \\
\text { specific version }\end{array}$ & $\begin{array}{l}\text { Overall attrition } \\
\text { rate: } \\
\text { NA } \\
\text { Study Quality: } \\
\text { Fair }\end{array}$ \\
\hline
\end{tabular}


Sexual Dysfunction associated with Second-Generation Antidepressants in Patients with Major Depressive Disorder - Results from a Systematic Review with Network Meta-Analysis. Drug Safety; U. Reichenpfader, G. Gartlehner, L.C. Morgan, A. Greenblatt, B. Nussbaumer, R. A. Hansen, M. Van Noord, L. Lux, and B. N. Gaynes. Corresponding author: U. Reichenpfader, Department for Evidence-based Medicine and Clinical Epidemiology, Krems, Austria; Department of Medical and Health Sciences - Division of Community Medicine, Linköping University, Sweden; ureichenpfader@hotmail.com

\begin{tabular}{|c|c|c|c|c|c|}
\hline $\begin{array}{c}\text { Study } \\
\text { Characteristics }\end{array}$ & $\begin{array}{l}\text { Research Objective } \\
\text { Study Design }\end{array}$ & $\begin{array}{l}\text { Inclusion/Exclusion } \\
\text { Outcome Measures }\end{array}$ & $\begin{array}{c}\text { Population } \\
\text { Characteristics }\end{array}$ & $\begin{array}{c}\text { Sexual Dysfunction (SD) } \\
\text { Outcomes/ Adverse } \\
\text { Events } \\
\end{array}$ & $\begin{array}{l}\text { Method used to } \\
\text { determine SD }\end{array}$ \\
\hline $\begin{array}{l}\text { Author, Year } \\
\text { Clayton et al., } \\
2006[12] \\
\text { Country and } \\
\text { Setting } \\
\text { United States, } \\
\text { multicenter } \\
\text { Funding } \\
\text { GlaxoSmithKline } \\
\text { Risk of Bias: } \\
\text { Fair }\end{array}$ & $\begin{array}{l}\text { Research objective } \\
\text { To compare effects on } \\
\text { sexual functioning and } \\
\text { antidepressant efficacy of } \\
\text { once-daily BUP XL and ESC } \\
\text { in adults with MDD. } \\
\text { Drugs, Doses, and Range } \\
\text { D1: BUP XL (150-450 mg } 1 \\
\quad \text { x daily): } 150 \text { mg } 1 \times \text { daily } \\
\quad \text { week } 1 \text { (low); } 300 \text { mg } 1 \\
\quad \text { x daily during weeks } 2 \\
\text { to } 4 \text { (medium); on week } \\
5 \text {, daily dose could be } \\
\quad \text { increased to } 450 \text { mg } \\
\text { (high) if additional } \\
\text { efficacy was desired } \\
\text { D2: ESC (10-20 mg } 1 \text { x } \\
\text { daily): } 10 \text { mg } 1 \text { x daily } \\
\text { during weeks } 1 \text { to } 4 \\
\text { (low); ESC dose could } \\
\text { be increased to } 20 \text { mg } 1 \\
\quad \text { x daily (medium) for } \\
\text { weeks } 5 \text { to } 8 \text { if } \\
\text { additional efficacy was } \\
\text { needed } \\
\text { D3: PBO } \\
\text { Fixed dose } \\
\text { No } \\
\text { Flexible dose } \\
\text { Yes } \\
\text { Dosages equivalent } \\
\text { No }\end{array}$ & $\begin{array}{l}\text { Inclusion criteria: } \\
\text { - Adults (age range): } \\
\geq 18 \text { years } \\
\text { - Diagnosed with MDD } \\
\text { according to DSM-III or } \\
\text {-IV } \\
\text { - HAM-D: HAM-D-17 } \\
\text { total score } \geq 19 \text { at } \\
\text { screening and on the } \\
\text { day of randomization to } \\
\text { treatment } \\
\text { - Currently experiencing } \\
\text { a MDE lasting } \geq 12 \\
\text { weeks and }<2 \text { years, } \\
\text { but were otherwise } \\
\text { healthy } \\
\text { - Normal orgasm function } \\
\text { as assessed by } \\
\text { investigator interview } \\
\text { and were willing to } \\
\text { discuss their sexual } \\
\text { functioning with } \\
\text { investigator and } \\
\text { engaged in sexual } \\
\text { activity leading to } \\
\text { orgasm at least once } \\
\text { every } 2 \text { weeks. Patients } \\
\text { who had a sexual } \\
\text { desire disorder were } \\
\text { eligible for study if } \\
\text { investigoator } \\
\text { considered it to be } \\
\text { secondary to MDE. }\end{array}$ & $\begin{array}{l}\text { Groups similar at } \\
\text { baseline } \\
\text { Yes } \\
\mathbf{n}= \\
\text { Pooled } \\
\text { D1: } 276 \\
\text { D2: } 281 \\
\text { D3: } 273 \\
\text { Mean age, years } \\
\text { Pooled } \\
\text { D1: } 37 \\
\text { D2: } 36 \\
\text { D3: } 36 \\
\text { Sex, \% female } \\
\text { Pooled } \\
\text { D1: } 58 \\
\text { D2: } 57 \\
\text { D3: } 60 \\
\text { Race, \% white } \\
\text { Pooled } \\
\text { D1: } 70 \\
\text { D2: } 68 \\
\text { D3: } 70 \\
\text { Baseline HAM-A } \\
\text { NR } \\
\text { Insomnia, \% } \\
\text { NR } \\
\text { Concomitant anergia, \% } \\
\text { NR } \\
\text { Experienced prior } \\
\text { Do }\end{array}$ & $\begin{array}{l}\text { D1: Orgasm Dysfunction: } \\
\text { 15\%; worsened sexual } \\
\text { function: } 20 \% \\
\text { D2: Orgasm Dysfunction: } \\
\text { 30\%; worsened sexual } \\
\text { function: } 36 \% \\
\text { D3: Orgasm Dysfunction: } \\
\text { 9\%; worsened sexual } \\
\text { function: } 15 \% \\
\text { Withdrawals due to } \\
\text { adverse events, \% } \\
\text { Pooled: } \\
\text { D1: } 6 \\
\text { D2: } 4 \\
\text { D3: } 5 \\
\text { Study } 1: \\
\text { D1: } 3 \\
\text { D2: } 5 \\
\text { D3: } 5 \\
\text { Study 2: } \\
\text { D1: } 10 \\
\text { D2: } 3 \\
\text { D3: } 5 \\
\text { Withdrawals due to lack } \\
\text { of efficacy, \% } \\
\text { D1: NR } \\
\text { D2: NR } \\
\text { D3: NR }\end{array}$ & CSFQ-14 \\
\hline
\end{tabular}


Sexual Dysfunction associated with Second-Generation Antidepressants in Patients with Major Depressive Disorder - Results from a Systematic Review with Network Meta-Analysis. Drug Safety; U. Reichenpfader, G. Gartlehner, L.C. Morgan, A. Greenblatt, B. Nussbaumer, R. A. Hansen, M. Van Noord, L. Lux, and B. N. Gaynes. Corresponding author: U. Reichenpfader, Department for Evidence-based Medicine and Clinical Epidemiology, Krems, Austria; Department of Medical and Health Sciences - Division of Community Medicine, Linköping University, Sweden; ureichenpfader@hotmail.com

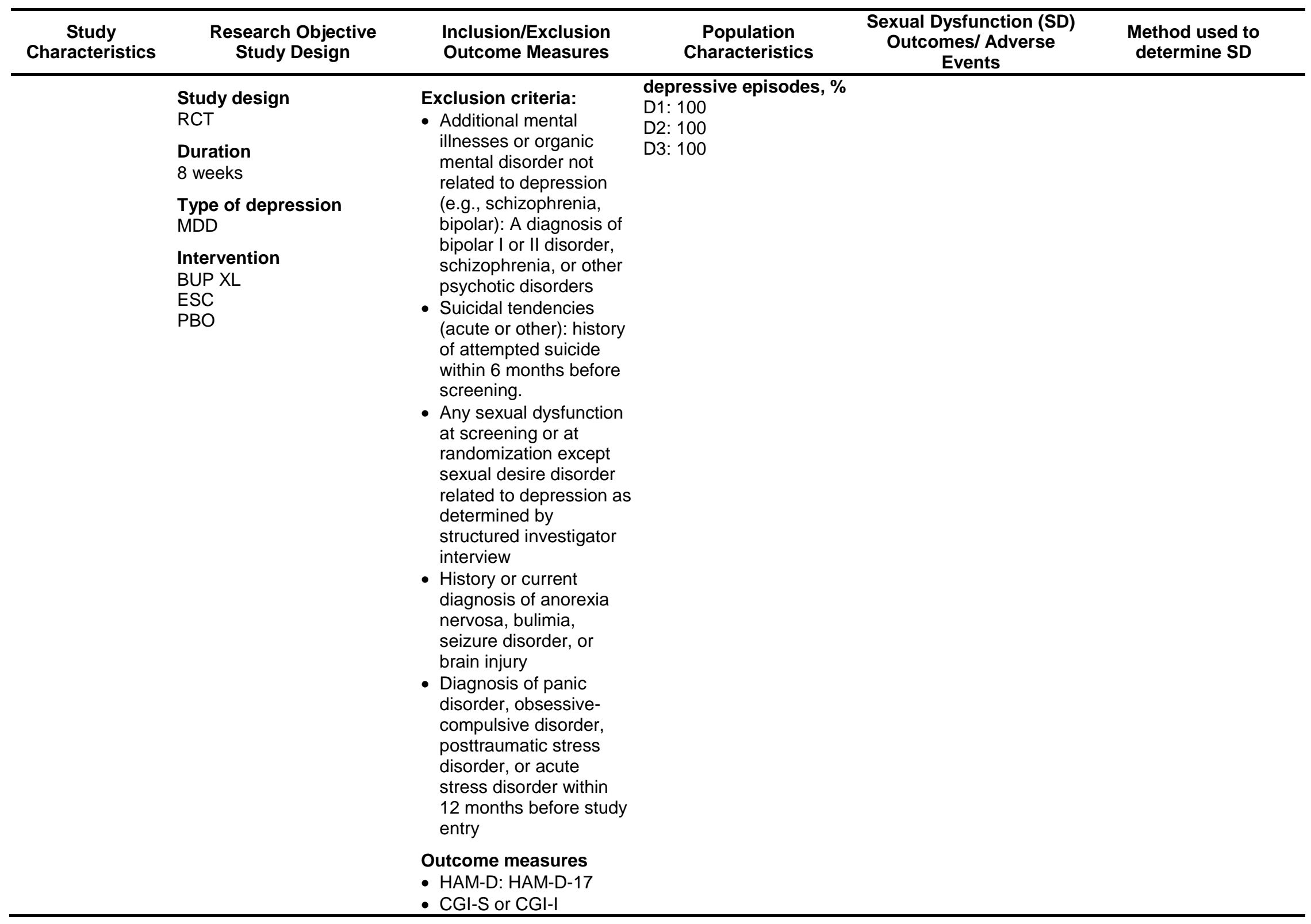


Sexual Dysfunction associated with Second-Generation Antidepressants in Patients with Major Depressive Disorder - Results from a Systematic Review with Network Meta-Analysis. Drug Safety; U. Reichenpfader, G. Gartlehner, L.C. Morgan, A. Greenblatt, B. Nussbaumer, R. A. Hansen, M. Van Noord, L. Lux, and B. N. Gaynes. Corresponding author: U. Reichenpfader, Department for Evidence-based Medicine and Clinical Epidemiology, Krems, Austria; Department of Medical and Health Sciences - Division of Community Medicine, Linköping University, Sweden; ureichenpfader@hotmail.com

\begin{tabular}{|c|c|c|c|c|c|}
\hline $\begin{array}{c}\text { Study } \\
\text { Characteristics }\end{array}$ & $\begin{array}{l}\text { Research Objective } \\
\text { Study Design }\end{array}$ & $\begin{array}{l}\text { Inclusion/Exclusion } \\
\text { Outcome Measures }\end{array}$ & $\begin{array}{c}\text { Population } \\
\text { Characteristics }\end{array}$ & $\begin{array}{c}\text { Sexual Dysfunction (SD) } \\
\text { Outcomes/ Adverse } \\
\text { Events }\end{array}$ & $\begin{array}{l}\text { Method used to } \\
\text { determine SD }\end{array}$ \\
\hline & & $\begin{array}{l}\text { CSFQ (secondary } \\
\text { endpoint) } \\
\text { HAD }\end{array}$ & & & \\
\hline
\end{tabular}


Sexual Dysfunction associated with Second-Generation Antidepressants in Patients with Major Depressive Disorder - Results from a Systematic Review with Network Meta-Analysis. Drug Safety; U. Reichenpfader, G. Gartlehner, L.C. Morgan, A. Greenblatt, B. Nussbaumer, R. A. Hansen, M. Van Noord, L. Lux, and B. N. Gaynes. Corresponding author: U. Reichenpfader, Department for Evidence-based Medicine and Clinical Epidemiology, Krems, Austria; Department of Medical and Health Sciences - Division of Community Medicine, Linköping University, Sweden; ureichenpfader@hotmail.com

\begin{tabular}{|c|c|c|c|c|c|}
\hline $\begin{array}{c}\text { Study } \\
\text { Characteristics }\end{array}$ & $\begin{array}{l}\text { Research Objective } \\
\text { Study Design }\end{array}$ & $\begin{array}{l}\text { Inclusion/Exclusion } \\
\text { Outcome Measures }\end{array}$ & $\begin{array}{c}\text { Population } \\
\text { Characteristics }\end{array}$ & $\begin{array}{c}\text { Sexual Dysfunction (SD) } \\
\text { Outcomes/ Adverse } \\
\text { Events }\end{array}$ & $\begin{array}{l}\text { Method used to } \\
\text { determine SD }\end{array}$ \\
\hline $\begin{array}{l}\text { Author, Year } \\
\text { Clayton et al., } \\
\text { 2007[13] } \\
\text { Country and } \\
\text { Setting } \\
\text { United States, } \\
\text { multicenter (36 } \\
\text { psychiatric clinical } \\
\text { settings) } \\
\text { Funding } \\
\text { Eli Lilly and } \\
\text { Company } \\
\text { Study Quality: } \\
\text { Good } \\
\text { Attrition rate, \%: } \\
\text { 8 weeks: } \\
\text { D1: } 31.9 \\
\text { D2: } 24.5 \\
\text { D3: } 29.9 \\
8 \text { months: } \\
\text { D1: } 63.7 \\
\text { D2: } 55.8 \\
\text { D3: } 89.8^{\star}\end{array}$ & $\begin{array}{l}\text { Research objective } \\
\text { Comparisons of changes in } \\
\text { sexual functioning for DUL } \\
\text { and ESC in which primary } \\
\text { objective was to compare } \\
\text { onset of antidepressant } \\
\text { action for DUL } 60 \text { mg/day } \\
\text { with that of ESC } 10 \text { mg/day. } \\
\text { secondary objection was to } \\
\text { compare differential drug } \\
\text { effects on sexual functioning } \\
\text { over acute and longer-term } \\
\text { course of study. } \\
\text { Drugs, Doses, and Range } \\
\text { D1: DUL (40-60 mg 1-2 x } \\
\text { daily): } 60 \text { mg/day } \\
\text { (medium) for initial } \\
\text { eight-week acute- } \\
\text { treatment phase; DUL } \\
\quad 60-120 \text { mg/day } \\
\text { (medium-high) during } \\
\text { extension phase } \\
\text { D2: ESC (10-20 mg } 1 \times \\
\text { daily): } 10 \text { mg/day (low) } \\
\text { for initial } 8 \text {-week acute- } \\
\text { treatment phase; } 10-20 \\
\text { mg/day (low-high) } \\
\text { during extension phase } \\
\text { D3: PBO } \\
\text { Fixed dose } \\
\text { Yes } \\
\text { Flexible dose } \\
\text { Yes } \\
\text { Dosages equivalent } \\
\text { No } \\
\text { Study design }\end{array}$ & $\begin{array}{l}\text { Inclusion criteria: } \\
\text { - Adults (age range): } \\
\geq 18 \text { years of age } \\
\text { - Diagnosed with MDD } \\
\text { according to DSM-III or } \\
\text {-IV } \\
\text { - MADRS: total score } \\
\geq 22 \\
\text { - CGI-S: } \geq 4 \\
\text { Exclusion criteria: } \\
\text { - Additional mental } \\
\text { illnesses or organic } \\
\text { mental disorder not } \\
\text { related to depression } \\
\text { (e.g., schizophrenia, } \\
\text { bipolar): Illicit drug and } \\
\text { alcohol abuse: history } \\
\text { of substance } \\
\text { dependence within past } \\
6 \text { months } \\
\text { - Clinically significant } \\
\text { medical disease: } \\
\text { Investigational drug use } \\
\text { within last: A history of } \\
\text { a lack of response, at } \\
\text { any time, to an } \\
\text { adequate trial of DUL } \\
\text { ( } \geq 60 \text { mg/day for } \geq 4 \\
\text { weeks), ESC ( } \geq 10 \\
\text { mg/day for } \geq 4 \text { weeks), } \\
\text { or CIT ( } \geq 20 \text { mg/day for } \\
\geq 4 \text { weeks) } \\
\text { - ECT or transcranial } \\
\text { magnetic stimulation } \\
\text { within past year } \\
\text { - Suicidal tendencies } \\
\text { (acute or other): serious } \\
\text { suicidal risk } \\
\text { - Any current primary }\end{array}$ & $\begin{array}{l}\text { Groups similar at } \\
\text { baseline } \\
\text { Yes } \\
\mathbf{n}= \\
\text { D1: } 273 \\
\text { D2: } 274 \\
\text { D3: } 137 \\
\text { Mean age, years } \\
\text { D1: } 41.1 \\
\text { D2: } 43.3 \\
\text { D3: } 42.5 \\
\text { Sex, \% female } \\
\text { D1: } 63.4 \\
\text { D2: } 67.9 \\
\text { D3: } 63.5 \\
\text { Race, \% white } \\
\text { D1: } 75.5 \\
\text { D2: } 77.4 \\
\text { D3: } 82.5 \\
\text { Baseline HAM-A } \\
\text { NR } \\
\text { Insomnia, \% } \\
\text { NR } \\
\text { Concomitant anergia, \% } \\
\text { NR } \\
\text { Experienced prior } \\
\text { depressive episodes, \% } \\
\text { NR } \\
\text { Comments: } \\
\text { The mean age of patients } \\
\text { in DUL treatment group } \\
\text { was significantly lower } \\
\text { than that in ESC (41.1 } \\
\text { years vs. } 43.3 \text { years; } P: \\
0.036) . \text { CGI-S means } \\
\text { D. }\end{array}$ & $\begin{array}{l}\text { Overall adverse events, } \\
\%: \\
\text { NR } \\
\text { At end point of acute- } \\
\text { treatment phase ( } 8 \text { weeks } \\
\text { or last observation), } \\
\text { categorical assessment of } \\
\text { changes in global sexual } \\
\text { functioning in DUL-treated } \\
\text { male patients showed that } \\
54.4 \% \text { reported } \\
\text { improvement, } 8.9 \% \\
\text { reported no change, and } \\
36.7 \% \text { reported } \\
\text { worsening; whereas in } \\
\text { ESC-treated male } \\
\text { patients, } 34.2 \% \text { reported } \\
\text { improvement, } 6.6 \% \\
\text { reported no change, and } \\
59.2 \% \text { reported worsening } \\
\text { ( } P=0.019 \text { DUL vs. ESC). }\end{array}$ & CSFQ-14 \\
\hline
\end{tabular}


Sexual Dysfunction associated with Second-Generation Antidepressants in Patients with Major Depressive Disorder - Results from a Systematic Review with Network Meta-Analysis. Drug Safety; U. Reichenpfader, G. Gartlehner, L.C. Morgan, A. Greenblatt, B. Nussbaumer, R. A. Hansen, M. Van Noord, L. Lux, and B. N. Gaynes. Corresponding author: U. Reichenpfader, Department for Evidence-based Medicine and Clinical Epidemiology, Krems, Austria; Department of Medical and Health Sciences - Division of Community Medicine, Linköping University, Sweden; ureichenpfader@hotmail.com

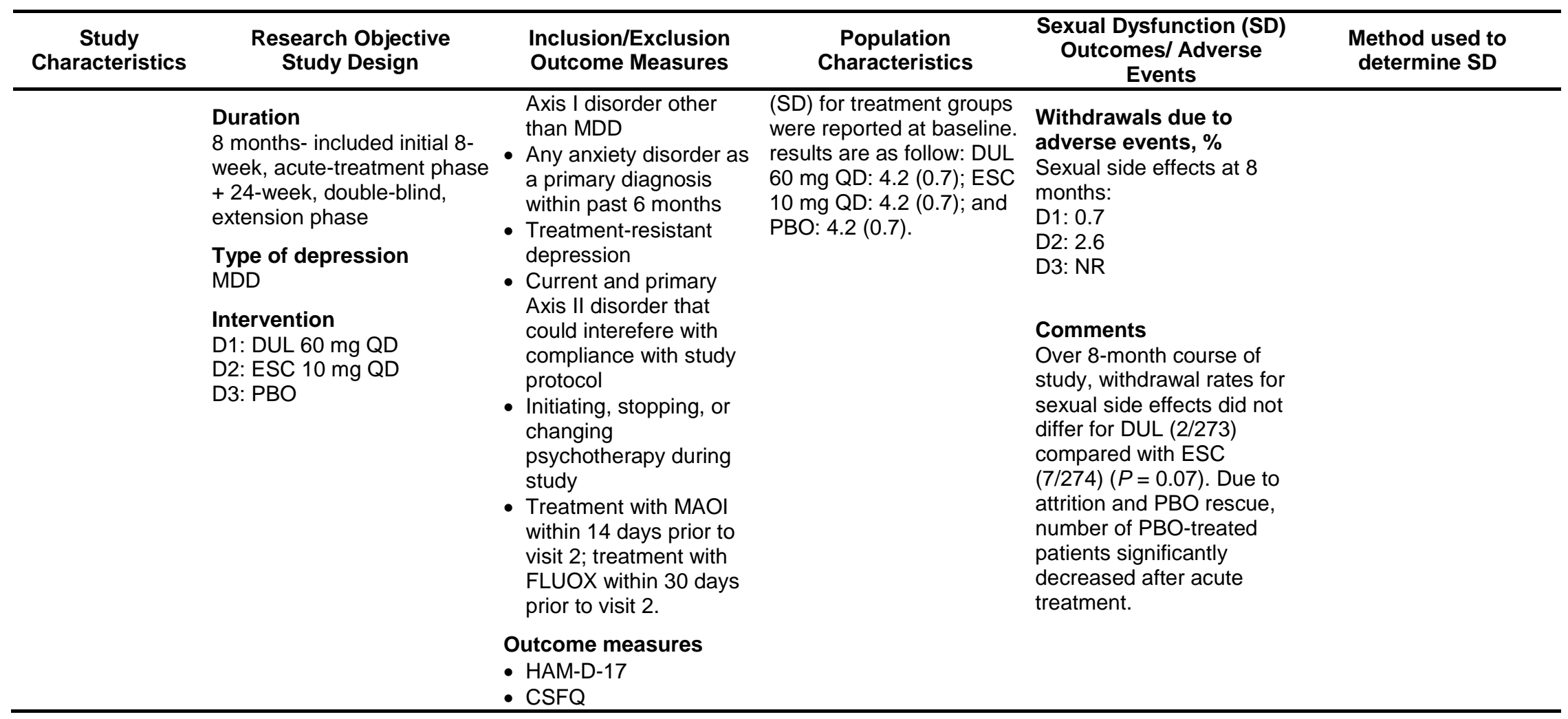


Sexual Dysfunction associated with Second-Generation Antidepressants in Patients with Major Depressive Disorder - Results from a Systematic Review with Network Meta-Analysis. Drug Safety; U. Reichenpfader, G. Gartlehner, L.C. Morgan, A. Greenblatt, B. Nussbaumer, R. A. Hansen, M. Van Noord, L. Lux, and B. N. Gaynes. Corresponding author: U. Reichenpfader, Department for Evidence-based Medicine and Clinical Epidemiology, Krems, Austria; Department of Medical and Health Sciences - Division of Community Medicine, Linköping University, Sweden; ureichenpfader@hotmail.com

\begin{tabular}{|c|c|c|c|c|c|c|}
\hline $\begin{array}{c}\text { Study } \\
\text { Characteristics }\end{array}$ & $\begin{array}{l}\text { Research Objective } \\
\text { Study Design }\end{array}$ & Inclusion/Exclusion & $\begin{array}{c}\text { Baseline } \\
\text { Characteristics }\end{array}$ & $\begin{array}{l}\text { Sexual Dysfunction } \\
\text { (SD) Outcomes/ } \\
\text { Adverse Events } \\
\end{array}$ & $\begin{array}{l}\text { Method used to } \\
\text { determine SD }\end{array}$ & $\begin{array}{c}\text { Analysis and Study } \\
\text { Quality (Risk of } \\
\text { Bias) }\end{array}$ \\
\hline $\begin{array}{l}\text { Author: } \\
\text { Coleman et al., } \\
\text { 1999[14] } \\
\text { Country and } \\
\text { setting: } \\
\text { United States } \\
\text { Multicenter (9 } \\
\text { centers) } \\
\text { Funding: } \\
\text { Glaxo Wellcome } \\
\text { Inc }\end{array}$ & $\begin{array}{l}\text { Research objective: } \\
\text { To compare sexual } \\
\text { functioning as well as } \\
\text { safety and efficacy of } \\
\text { BUP SR and SER } \\
\text { Duration of study: } \\
8 \text { wks } \\
\text { Study design: } \\
\text { RCT } \\
\text { Overall study N: } \\
240 \\
\text { Intervention: } \\
\text { D1: SER: } 50-200 \mathrm{mg} / \mathrm{d} \\
\text { D2: BUP: } 150-400 \\
\quad \text { mg/d } \\
\text { D3: PBO }\end{array}$ & $\begin{array}{l}\text { Inclusion criteria: } \\
\text { - Adults } 18+ \\
\text { - Diagnosed with } \\
\text { MDD according to } \\
\text { DSM-III or -IV } \\
\text { - Minimum HAM-D } \\
\text { score of } 18 \\
\text { - Be in a stable } \\
\text { relationship, have } \\
\text { normal sexual } \\
\text { functioning, and } \\
\text { sexual activity at } \\
\text { least once every } 2 \\
\text { wks } \\
\text { - Currently } \\
\text { experiencing } \\
\text { recurrent major } \\
\text { episode of } \\
\text { depression } \\
\text { Exclusion criteria: } \\
\text { - Pregnant } \\
\text { - Lactating } \\
\text { - Concomitant } \\
\text { psychotheraputic or } \\
\text { psychotropic } \\
\text { medications } \\
\text { - Illicit drug and } \\
\text { alcohol abuse } \\
\text { - Suicidal tendencies }\end{array}$ & $\begin{array}{l}\text { Mean age (yrs): } \\
\text { D1: } 38.3 \\
\text { D2: } 38.1 \\
\text { D3: } 38.5 \\
\text { Sex (\% female): } \\
\text { D1: } 54 \\
\text { D2: } 56 \\
\text { D3: } 59 \\
\text { Race (\% white): } \\
\text { D1: } 92 \\
\text { D2: } 87 \\
\text { D3: } 88 \\
\text { Baseline (HAM-A): } \\
\text { NR } \\
\text { Baseline HAM-D: } \\
\text { D1: } 34.5 \\
\text { D2: } 34.8 \\
\text { D3: } 34.0\end{array}$ & $\begin{array}{l}\text { SD at } 8 \text { weeks: } \\
\text { D1: Sexual Arousal } \\
\text { Disorder: 9\%; Orgasm } \\
\text { Dysfunction: } 36 \% \\
\text { D2: Sexual Arousal } \\
\text { Disorder: 6\%; Orgasm } \\
\text { Dysfunction: 10\%; } \\
\text { total SD 16\% } \\
\text { D3: Sexual Arousal } \\
\text { Disorder: 10\%); } \\
\text { Orgasm Dysfunction: } \\
\text { 5\%); total SD } \\
\text { (excluding sexual } \\
\text { desire disorder): } 15 \%\end{array}$ & $\begin{array}{l}\text { structured clinical } \\
\text { interview (only sexually } \\
\text { active included); } \\
\text { diagnosis based on } \\
\text { DSM-IV criteria }\end{array}$ & $\begin{array}{l}\text { Overall attrition } \\
\text { rate: } \\
30 \% \\
\text { ITT Analysis } \\
\text { Yes } \\
\text { Study Quality: } \\
\text { Fair }\end{array}$ \\
\hline
\end{tabular}


Sexual Dysfunction associated with Second-Generation Antidepressants in Patients with Major Depressive Disorder - Results from a Systematic Review with Network Meta-Analysis. Drug Safety; U. Reichenpfader, G. Gartlehner, L.C. Morgan, A. Greenblatt, B. Nussbaumer, R. A. Hansen, M. Van Noord, L. Lux, and B. N. Gaynes. Corresponding author: U. Reichenpfader, Department for Evidence-based Medicine and Clinical Epidemiology, Krems, Austria; Department of Medical and Health Sciences - Division of Community Medicine,

Linköping University, Sweden; ureichenpfader@hotmail.com

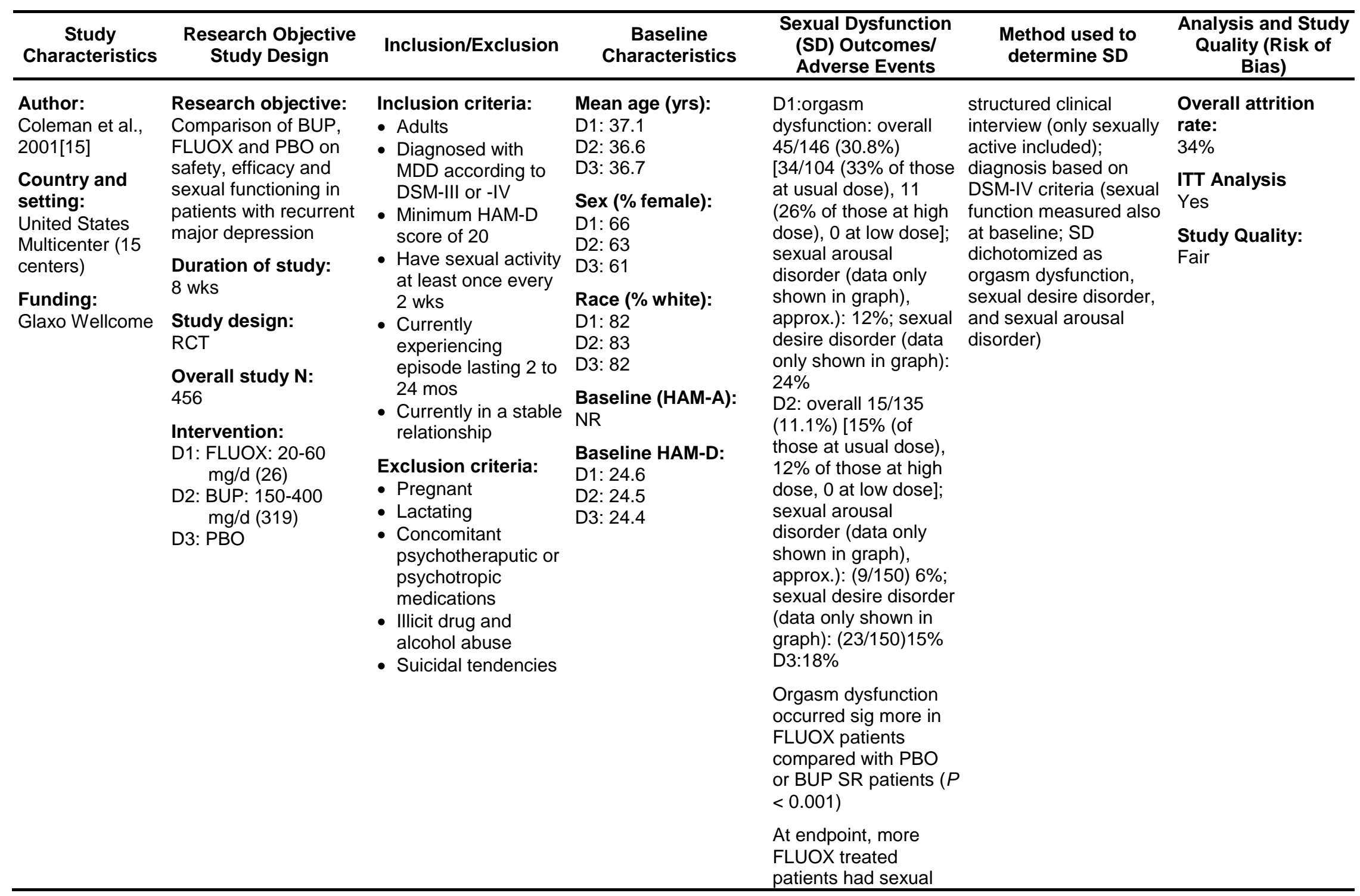


Sexual Dysfunction associated with Second-Generation Antidepressants in Patients with Major Depressive Disorder - Results from a Systematic Review with Network Meta-Analysis. Drug Safety; U. Reichenpfader, G. Gartlehner, L.C. Morgan, A. Greenblatt, B. Nussbaumer, R. A. Hansen, M. Van Noord, L. Lux, and B. N. Gaynes. Corresponding author: U. Reichenpfader, Department for Evidence-based Medicine and Clinical Epidemiology, Krems, Austria; Department of Medical and Health Sciences - Division of Community Medicine, Linköping University, Sweden; ureichenpfader@hotmail.com

desire disorder than

BUP SR treated

patients

$(P<0.05)$

Sig more buproion

SR-treated patients

were satisfied with

sexual function

(analysis only for

patients satisfied at

baseline; no data

reported) $P<0.05$

Compliance: $96.8 \%$ to

$98.8 \%$ in all groups

\begin{tabular}{|c|c|c|c|c|c|c|}
\hline $\begin{array}{c}\text { Study } \\
\text { Characteristics }\end{array}$ & $\begin{array}{l}\text { Research Objective } \\
\text { Study Design }\end{array}$ & Inclusion/Exclusion & $\begin{array}{c}\text { Baseline } \\
\text { Characteristics }\end{array}$ & $\begin{array}{l}\text { Sexual Dysfunction } \\
\text { (SD) Outcomes/ } \\
\text { Adverse Events }\end{array}$ & $\begin{array}{l}\text { Method used to } \\
\text { determine SD }\end{array}$ & $\begin{array}{c}\text { Analysis and Study } \\
\text { Quality (Risk of } \\
\text { Bias) }\end{array}$ \\
\hline $\begin{array}{l}\text { Author: } \\
\text { Croft et al., } \\
\text { 1999[16] } \\
\text { Country and } \\
\text { setting: } \\
\text { United States } \\
\text { Multicenter (8 } \\
\text { centers) } \\
\text { Funding: } \\
\text { Glaxo Wellcome }\end{array}$ & $\begin{array}{l}\text { Research objective: } \\
\text { Comparison of efficacy } \\
\text { and effects on sexual } \\
\text { functioning of } \\
\text { depressed patients } \\
\text { using BUP, SER, or } \\
\text { PBO } \\
\text { Duration of study: } \\
8 \text { wks } \\
\text { Study design: } \\
\text { RCT } \\
\text { Overall study N: } \\
239 \\
\text { Intervention: } \\
\text { D1: SER: } 50-200 \text { mg/d } \\
\text { (mean = } 121 \text { ) } \\
\text { D2: BUP: } 150-400 \\
\text { mg/d (mean = 293) }\end{array}$ & $\begin{array}{l}\text { Inclusion criteria: } \\
\text { - Adults } 18 \text { and over } \\
\text { - Diagnosed with } \\
\text { MDD according to } \\
\text { DSM-III or -IV } \\
\text { - Minimum HAM-D } \\
\text { score of } 18 \\
\text { - In stable } \\
\text { relationship } \\
\text { - Have normal sexual } \\
\text { functioning and } \\
\text { sexual activity at } \\
\text { least once every } 2 \\
\text { wks } \\
\text { - Current depressive } \\
\text { episode of } 8 \text { wks to } \\
24 \text { mos } \\
\text { Exclusion criteria: } \\
\text { - Pregnant }\end{array}$ & $\begin{array}{l}\text { Mean age (yrs): } \\
\text { D1: } 36.0 \\
\text { D2: } 35.9 \\
\text { D3: } 37.4 \\
\text { Sex (\% female): } \\
\text { D1: } 50 \\
\text { D2: } 51 \\
\text { D3: } 50 \\
\text { Race (\% white): } \\
\text { D1: } 87 \\
\text { D2: } 86 \\
\text { D3: } 88 \\
\text { Baseline (HAM-A): } \\
\text { NR } \\
\text { Baseline HAM-D: } \\
\text { NR }\end{array}$ & $\begin{array}{l}\text { SD day } 56: \\
\text { D1: Sexual desire } \\
\text { disorder: } 28 \% \text { (from } \\
\text { baseline } 43 \% \text { ); sexual } \\
\text { arousal disorder: } 6 \% \\
\text { (approx., data not } \\
\text { reported, only shown } \\
\text { in graph); orgasmic } \\
\text { dysfunction (orgasm } \\
\text { delay or failure); } 41 \% \\
\text { (approx., data not } \\
\text { reported, only shown } \\
\text { in graph } \\
\text { D2: Sexual desire } \\
\text { disorder: } 19 \% \text { (from } \\
\text { baseline } 39 \% \text { ); sexual } \\
\text { arousal disorder: } 6 \% \\
\text { (approx., data not } \\
\text { reported, only shown } \\
\text { in graph); orgasmic }\end{array}$ & $\begin{array}{l}\text { structured clinical } \\
\text { interview, diagnosis } \\
\text { according to DSM-IV } \\
\text { definitions of SD; only } \\
\text { sexually active \& free of } \\
\text { SD included }\end{array}$ & $\begin{array}{l}\text { Overall attrition } \\
\text { rate: } \\
32 \% \\
\text { ITT Analysis } \\
\text { Yes } \\
\text { Study Quality: } \\
\text { Fair }\end{array}$ \\
\hline
\end{tabular}


Sexual Dysfunction associated with Second-Generation Antidepressants in Patients with Major Depressive Disorder - Results from a Systematic Review with Network Meta-Analysis. Drug Safety; U. Reichenpfader, G. Gartlehner, L.C. Morgan, A. Greenblatt, B. Nussbaumer, R. A. Hansen, M. Van Noord, L. Lux, and B. N. Gaynes. Corresponding author: U. Reichenpfader, Department for Evidence-based Medicine and Clinical Epidemiology, Krems, Austria; Department of Medical and Health Sciences - Division of Community Medicine, Linköping University, Sweden; ureichenpfader@hotmail.com

\begin{tabular}{|c|c|c|}
\hline D3: PBO & $\begin{array}{l}\text { - Lactating } \\
\text { - Concomitant } \\
\text { psychotheraputic or } \\
\text { psychotropic } \\
\text { medications } \\
\text { - Illicit drug and } \\
\text { alcohol abuse } \\
\text { - Suicidal tendencies }\end{array}$ & $\begin{array}{l}\text { dysfunction (orgasm } \\
\text { delay or failure): } 15 \% \\
\text { (approx., data not } \\
\text { reported, only shown } \\
\text { in graph) } \\
\text { D3: no data reported, } \\
\text { only from graph: } \\
10.5 \% \text { (Sexual } \\
\text { Arousal Disorder: } \\
1.5 \% \text {; Orgasm } \\
\text { Dysfunction: } 9 \% \text { ) }\end{array}$ \\
\hline & & $\begin{array}{l}\text { both BUP and SER } \\
\text { had higher sexual } \\
\text { arousal disorder }(P< \\
0.05) \text { than PBO }\end{array}$ \\
\hline & & $\begin{array}{l}\text { Orgasmic dysfunction } \\
\text { occurred sig more in } \\
\text { SER patients } \\
\text { compared with PBO } \\
\text { or BUP patients }(P< \\
0.001)\end{array}$ \\
\hline
\end{tabular}


Sexual Dysfunction associated with Second-Generation Antidepressants in Patients with Major Depressive Disorder - Results from a Systematic Review with Network Meta-Analysis. Drug Safety; U. Reichenpfader, G. Gartlehner, L.C. Morgan, A. Greenblatt, B. Nussbaumer, R. A. Hansen, M. Van Noord, L. Lux, and B. N. Gaynes. Corresponding author: U. Reichenpfader, Department for Evidence-based Medicine and Clinical Epidemiology, Krems, Austria; Department of Medical and Health Sciences - Division of Community Medicine, Linköping University, Sweden; ureichenpfader@hotmail.com

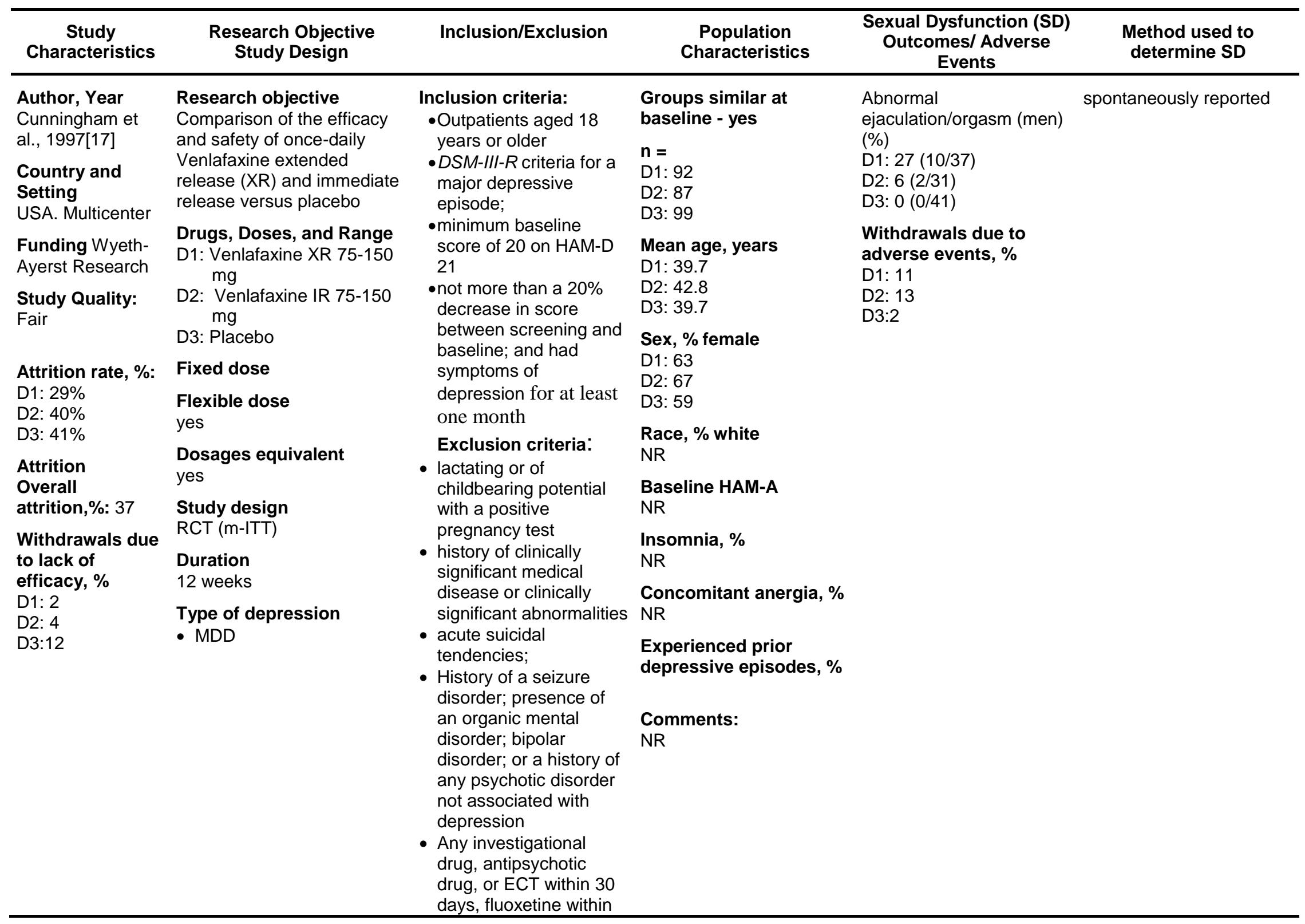


Sexual Dysfunction associated with Second-Generation Antidepressants in Patients with Major Depressive Disorder - Results from a Systematic Review with Network Meta-Analysis. Drug Safety; U. Reichenpfader, G. Gartlehner, L.C. Morgan, A. Greenblatt, B. Nussbaumer, R. A. Hansen, M. Van Noord, L. Lux, and B. N. Gaynes. Corresponding author: U. Reichenpfader, Department for Evidence-based Medicine and Clinical Epidemiology, Krems, Austria; Department of Medical and Health Sciences - Division of Community Medicine, Linköping University, Sweden; ureichenpfader@hotmail.com

\begin{tabular}{|c|c|c|c|c|c|}
\hline $\begin{array}{c}\text { Study } \\
\text { Characteristics }\end{array}$ & $\begin{array}{l}\text { Research Objective } \\
\text { Study Design }\end{array}$ & Inclusion/Exclusion & $\begin{array}{c}\text { Population } \\
\text { Characteristics }\end{array}$ & $\begin{array}{c}\text { Sexual Dysfunction (SD) } \\
\text { Outcomes/ Adverse } \\
\text { Events }\end{array}$ & $\begin{array}{l}\text { Method used to } \\
\text { determine SD }\end{array}$ \\
\hline & & $\begin{array}{l}21 \text { days, or monoamine } \\
\text { oxidase } \\
\text { inhibitor,,paroxetine, or } \\
\text { sertraline within } 14 \\
\text { days, or use of any } \\
\text { other antidepressant, } \\
\text { anxiolytic, sedative- } \\
\text { hypnotic drug, or } \\
\text { psychotropic drug or } \\
\text { substance within } 7 \text { days } \\
\text { - any nonpsychotropic } \\
\text { drug with psychotropic } \\
\text { effects unless the } \\
\text { dosage had been stable } \\
\text { for a minimum of one } \\
\text { month }\end{array}$ & & & \\
\hline
\end{tabular}


Sexual Dysfunction associated with Second-Generation Antidepressants in Patients with Major Depressive Disorder - Results from a Systematic Review with Network Meta-Analysis. Drug Safety; U. Reichenpfader, G. Gartlehner, L.C. Morgan, A. Greenblatt, B. Nussbaumer, R. A. Hansen, M. Van Noord, L. Lux, and B. N. Gaynes. Corresponding author: U. Reichenpfader, Department for Evidence-based Medicine and Clinical Epidemiology, Krems, Austria; Department of Medical and Health Sciences - Division of Community Medicine, Linköping University, Sweden; ureichenpfader@hotmail.com

\begin{tabular}{|c|c|c|c|c|c|c|}
\hline $\begin{array}{c}\text { Study } \\
\text { Characteristics }\end{array}$ & $\begin{array}{l}\text { Research Objective } \\
\text { Study Design }\end{array}$ & Inclusion/Exclusion & $\begin{array}{c}\text { Baseline } \\
\text { Characteristics }\end{array}$ & $\begin{array}{l}\text { Sexual Dysfunction } \\
\text { (SD) Outcomes/ } \\
\text { Adverse Events }\end{array}$ & $\begin{array}{l}\text { Method used to } \\
\text { determine SD }\end{array}$ & $\begin{array}{c}\text { Analysis and Study } \\
\text { Quality (Risk of } \\
\text { Bias) }\end{array}$ \\
\hline $\begin{array}{l}\text { Author: } \\
\text { Dalery and Honig } \\
2003[18] \\
\text { Country and } \\
\text { setting: } \\
\text { Europe } \\
\text { Multicenter } \\
\text { Funding: } \\
\text { Solvay } \\
\text { Pharmaceuticals }\end{array}$ & $\begin{array}{l}\text { Research objective: } \\
\text { Comparison of efficacy } \\
\text { and safety of FLUV and } \\
\text { FLUOX } \\
\text { Duration of study: } \\
6 \text { wks } \\
\text { Study design: } \\
\text { RCT } \\
\text { Overall study } \mathrm{N}: \\
184 \\
\text { Intervention: } \\
\text { D1: FLUOX: } 20 \mathrm{mg} / \mathrm{d} \\
\text { D2: FLUV: } 100 \mathrm{mg} / \mathrm{d}\end{array}$ & $\begin{array}{l}\text { Inclusion criteria: } \\
\text { - Adults } 18 \text { to } 70 \\
\text { - Diagnosed with } \\
\text { MDD according to } \\
\text { DSM-III or -IV } \\
\text { - Minimum HAM-D } \\
\text { score of } \geq 17 \\
\text { Exclusion criteria: } \\
\text { - Pregnant } \\
\text { - Lactating } \\
\text { - Concomitant } \\
\text { psychotheraputic or } \\
\text { psychotropic } \\
\text { medications } \\
\text { - Additional mental } \\
\text { illnesses or organic } \\
\text { mental disorder } \\
\text { - Illicit drug and } \\
\text { alcohol abuse } \\
\text { - Suicidal tendencies }\end{array}$ & $\begin{array}{l}\text { Mean age (yrs): } \\
\text { D1: } 42.0 \\
\text { D2: } 42.1 \\
\text { Sex (\% female): } \\
\text { D1: } 63.3 \\
\text { D2: } 62.7 \\
\text { Race (\% white): } \\
\text { NR } \\
\text { Baseline (HAM-A): } \\
\text { NR } \\
\text { Mean HAM-D score } \\
\text { at baseline: } \\
\text { D1: } 22.3 \\
\text { D2: } 22.2\end{array}$ & $\begin{array}{l}\text { D1: ejaculatory } \\
\text { dysfunction, erectile } \\
\text { dysfunction: } 2 / 35 \\
\text { ( } 5.7 \% \text { of men) } \\
\text { D2: ejaculatory } \\
\text { dysfunction } 1 / 33 \text { (3\% } \\
\text { of men) }\end{array}$ & $\mathrm{NR}$ & $\begin{array}{l}\text { Overall attrition } \\
\text { rate: } \\
20.9 \% \\
\text { ITT analysis: } \\
\text { Yes } \\
\text { Study Quality: } \\
\text { Fair }\end{array}$ \\
\hline
\end{tabular}


Sexual Dysfunction associated with Second-Generation Antidepressants in Patients with Major Depressive Disorder - Results from a Systematic Review with Network Meta-Analysis. Drug Safety; U. Reichenpfader, G. Gartlehner, L.C. Morgan, A. Greenblatt, B. Nussbaumer, R. A. Hansen, M. Van Noord, L. Lux, and B. N. Gaynes. Corresponding author: U. Reichenpfader, Department for Evidence-based Medicine and Clinical Epidemiology, Krems, Austria; Department of Medical and Health Sciences - Division of Community Medicine, Linköping University, Sweden; ureichenpfader@hotmail.com

\begin{tabular}{|c|c|c|c|c|c|c|}
\hline $\begin{array}{c}\text { Study } \\
\text { Characteristics }\end{array}$ & $\begin{array}{l}\text { Research Objective } \\
\text { Study Design }\end{array}$ & Inclusion/Exclusion & $\begin{array}{c}\text { Baseline } \\
\text { Characteristics }\end{array}$ & $\begin{array}{l}\text { Sexual Dysfunction } \\
\text { (SD) Outcomes/ } \\
\text { Adverse Events }\end{array}$ & $\begin{array}{l}\text { Method used to } \\
\text { determine SD }\end{array}$ & $\begin{array}{c}\text { Analysis and Study } \\
\text { Quality (Risk of } \\
\text { Bias) }\end{array}$ \\
\hline
\end{tabular}


Sexual Dysfunction associated with Second-Generation Antidepressants in Patients with Major Depressive Disorder - Results from a Systematic Review with Network Meta-Analysis. Drug Safety; U. Reichenpfader, G. Gartlehner, L.C. Morgan, A. Greenblatt, B. Nussbaumer, R. A. Hansen, M. Van Noord, L. Lux, and B. N. Gaynes. Corresponding author: U. Reichenpfader, Department for Evidence-based Medicine and Clinical Epidemiology, Krems, Austria; Department of Medical and Health Sciences - Division of Community Medicine, Linköping University, Sweden; ureichenpfader@hotmail.com

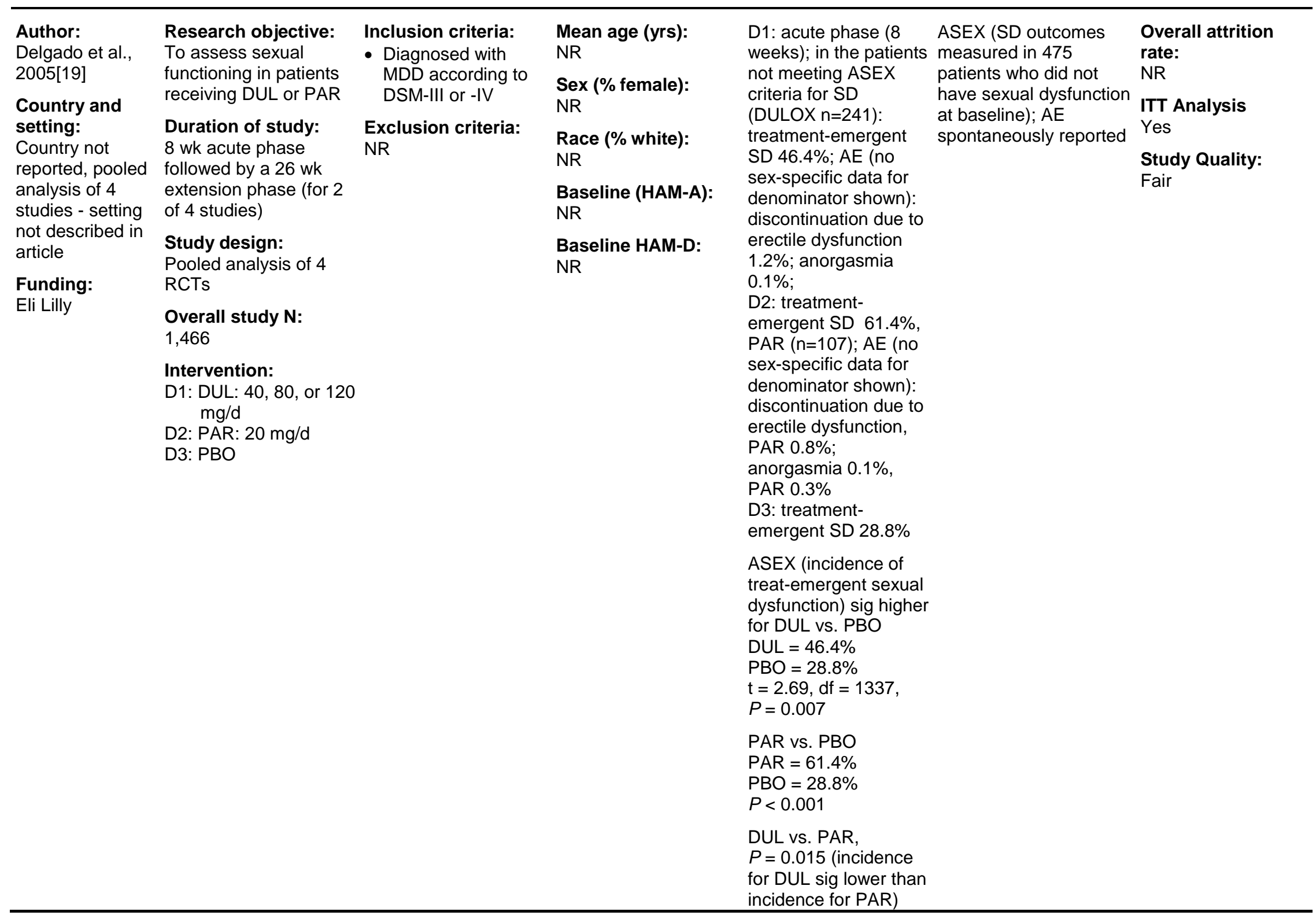


Sexual Dysfunction associated with Second-Generation Antidepressants in Patients with Major Depressive Disorder - Results from a Systematic Review with Network Meta-Analysis. Drug Safety; U. Reichenpfader, G. Gartlehner, L.C. Morgan, A. Greenblatt, B. Nussbaumer, R. A. Hansen, M. Van Noord, L. Lux, and B. N. Gaynes. Corresponding author: U. Reichenpfader, Department for Evidence-based Medicine and Clinical Epidemiology, Krems, Austria; Department of Medical and Health Sciences - Division of Community Medicine, Linköping University, Sweden; ureichenpfader@hotmail.com

\begin{tabular}{|c|c|c|c|c|c|c|}
\hline $\begin{array}{c}\text { Study } \\
\text { Characteristics }\end{array}$ & $\begin{array}{l}\text { Research Objective } \\
\text { Study Design }\end{array}$ & Inclusion/Exclusion & $\begin{array}{c}\text { Baseline } \\
\text { Characteristics }\end{array}$ & $\begin{array}{c}\text { Sexual Dysfunction } \\
\text { (SD) Outcomes/ } \\
\text { Adverse Events } \\
\end{array}$ & $\begin{array}{l}\text { Method used to } \\
\text { determine SD }\end{array}$ & $\begin{array}{c}\text { Analysis and Study } \\
\text { Quality (Risk of } \\
\text { Bias) }\end{array}$ \\
\hline \multirow{3}{*}{$\begin{array}{l}\text { Author: } \\
\text { Detke et al., } \\
\text { 2002[20] } \\
\text { Country and } \\
\text { setting: } \\
\text { United States, } \\
\text { multicenter (18 } \\
\text { sites) }\end{array}$} & \multirow{3}{*}{$\begin{array}{l}\text { Research objective: } \\
\text { To evaluate efficacy of } \\
\text { DUL vs. PBO for } \\
\text { treatment of MDD and } \\
\text { associated painful } \\
\text { symptoms } \\
\text { Duration of study: } \\
9 \text { wks }\end{array}$} & \multirow{3}{*}{$\begin{array}{l}\text { Inclusion criteria: } \\
\text { - Adults } 18 \text { or older } \\
\text { - MDD according to } \\
\text { DSM-IV } \\
\text { - Minimum HAM-D-17 } \\
\text { score of } 15 \\
\text { - Other: CGI-S of } 4 \text { or } \\
\text { more }\end{array}$} & \multirow{4}{*}{$\begin{array}{l}\text { Mean age (yrs): } \\
\text { D1: } 42.44 \\
\text { D2: } 42.34 \\
\text { Sex (\% female): } \\
\text { D1: } 65.0 \\
\text { D2: } 68.0 \\
\text { Race (\% white): } \\
\text { D1: } 87.0 \\
\text { D2: } 84.4\end{array}$} & \multirow{8}{*}{$\begin{array}{l}\text { Discontinuation due } \\
\text { to SD: } \\
\text { D1: } 3 / 45(6.7 \% \text { of } \\
\text { men) of DUL-treated } \\
\text { men dropped out due } \\
\text { to abnormal } \\
\text { ejaculation }\end{array}$} & \multirow{8}{*}{ spontaneously reported } & \multirow{8}{*}{$\begin{array}{l}\text { Overall attrition } \\
\text { rate: } \\
\text { NR } \\
\text { ITT Analysis } \\
\text { Yes } \\
\text { Study Quality: } \\
\text { Fair }\end{array}$} \\
\hline & & & & & & \\
\hline & & & & & & \\
\hline \multirow{5}{*}{$\begin{array}{l}\text { Funding: } \\
\text { Eli Lilly and } \\
\text { Company }\end{array}$} & \multirow{2}{*}{$\begin{array}{l}\text { Study design: } \\
\text { RCT }\end{array}$} & \multirow{5}{*}{$\begin{array}{l}\text { Note: Painful } \\
\text { symptoms not } \\
\text { required for inclusion } \\
\text { Exclusion criteria: } \\
\text { - Additional mental } \\
\text { illness or organic } \\
\text { mental disorder } \\
\text { - Psychotherapy } \\
\text { within } 6 \text { wks } \\
\text { - Substance abuse or } \\
\text { dependence (within } \\
1 \text { yr) } \\
\text { - Clinically sig } \\
\text { medical disease } \\
\text { - Treatment resistant } \\
\text { depression or lack } \\
\text { of response of } \\
\text { current MDD } \\
\text { episode to } 2 \text { prior } \\
\text { courses of therapy }\end{array}$} & & & & \\
\hline & & & Baseline HAM-D-17: & & & \\
\hline & $\begin{array}{l}\text { Overall study N: } \\
245\end{array}$ & & $\begin{array}{l}\text { D1: } 21.42(4.11) \\
\text { D2: } 21.14(3.72)\end{array}$ & & & \\
\hline & $\begin{array}{l}\text { Intervention: } \\
\text { D1: DUL } 60 \mathrm{mg} / \mathrm{d} \\
\text { D2: PBO }\end{array}$ & & $\begin{array}{l}\text { Baseline } 100 \mathrm{~mm} \\
\text { VAS (overall pain): } \\
\text { D1: } 29.02(25.10) \\
\text { D2: } 28.16(23.21)\end{array}$ & & & \\
\hline & & & $\begin{array}{l}\text { Baseline HAM-A: } \\
\text { NR }\end{array}$ & & & \\
\hline
\end{tabular}


Sexual Dysfunction associated with Second-Generation Antidepressants in Patients with Major Depressive Disorder - Results from a Systematic Review with Network Meta-Analysis. Drug Safety; U. Reichenpfader, G. Gartlehner, L.C. Morgan, A. Greenblatt, B. Nussbaumer, R. A. Hansen, M. Van Noord, L. Lux, and B. N. Gaynes. Corresponding author: U. Reichenpfader, Department for Evidence-based Medicine and Clinical Epidemiology, Krems, Austria; Department of Medical and Health Sciences - Division of Community Medicine, Linköping University, Sweden; ureichenpfader@hotmail.com

\begin{tabular}{|c|c|c|c|c|c|c|}
\hline $\begin{array}{c}\text { Study } \\
\text { Characteristics }\end{array}$ & $\begin{array}{c}\text { Research Objective } \\
\text { Duration } \\
\text { Study Design } \\
\end{array}$ & Inclusion/Exclusion & $\begin{array}{c}\text { Baseline } \\
\text { Characteristics }\end{array}$ & $\begin{array}{c}\text { Sexual Dysfunction } \\
\text { (SD) Outcomes/ } \\
\text { Adverse Events } \\
\end{array}$ & $\begin{array}{l}\text { Method used to } \\
\text { determine SD }\end{array}$ & $\begin{array}{c}\text { Analysis and Study } \\
\text { Quality (Risk of } \\
\text { Bias) }\end{array}$ \\
\hline $\begin{array}{l}\text { Author: } \\
\text { Detke et al., } \\
2004[21] \\
\text { Country and } \\
\text { setting: } \\
\text { United States } \\
\text { Multicenter, } \\
\text { university clinics } \\
\text { Funding: } \\
\text { Eli Lilly }\end{array}$ & $\begin{array}{l}\text { Research objective: } \\
\text { To determine } \\
\text { comparative efficacy } \\
\text { and safety of DUL and } \\
\text { PAR for treatment of } \\
\text { MDD } \\
\text { Duration of study: } \\
8 \text { wks } \\
\text { Study design: } \\
\text { RCT } \\
\text { Overall study } \mathrm{N}: \\
\text { 274 } \\
\text { Intervention: } \\
\text { D1: DUL } 80 \mathrm{mg} / \mathrm{d} \\
\text { D2: DUL } 120 \mathrm{mg} / \mathrm{d} \\
\text { D3: PAR: } 20 \mathrm{mg} / \mathrm{d} \\
\text { D4: PBO }\end{array}$ & $\begin{array}{l}\text { Inclusion criteria: } \\
\text { - Adults } 18 \text { or older } \\
\text { - Met DSM-IV and } \\
\text { MINI criteria for } \\
\text { MDD } \\
\text { - CGI-S rating > } 4 \\
\text { - Diagnosed with } \\
\text { MDD according to } \\
\text { DSM-III or -IV } \\
\text { - Minimum HAM-D } \\
\text { score of } 15 \\
\text { Exclusion criteria: } \\
\text { - Pregnant } \\
\text { - Additional mental } \\
\text { illnesses or organic } \\
\text { mental disorder } \\
\text { - Illicit drug and } \\
\text { alcohol abuse } \\
\text { - Clinically sig } \\
\text { medical disease } \\
\text { - Suicidal tendencies }\end{array}$ & $\begin{array}{l}\text { Mean age (yrs): } \\
\text { D1: } 43.1 \\
\text { D2: } 44.7 \\
\text { D3: } 42.0 \\
\text { D4: } 42.0 \\
\text { Sex (\% female): } \\
\text { D1: } 70 \\
\text { D2: } 70 \\
\text { D3: } 58 \\
\text { D4: } 58 \\
\text { Race (\% white): } \\
\text { D1: } 95 \\
\text { D2: } 92 \\
\text { D3: } 86 \\
\text { D4: } 86 \\
\text { Baseline (HAM-A): } \\
\text { D1: } 17.8 \\
\text { D2: } 18.0 \\
\text { D3: } 18.5 \\
\text { D4: } 17.9 \\
\text { Mean HAM-D score } \\
\text { at baseline: } \\
\text { D1: } 19.9 \text { (3.6) } \\
\text { D2: } 20.2(3.4) \\
\text { D3: } 20.3 \text { (4.1) } \\
\text { D4: } 19.9\end{array}$ & $\begin{array}{l}\text { treatment-emergent } \\
\text { SD (ASEX): \% } \\
\text { D1: } 46.5 \\
\text { D2: } 46.5 \\
\text { D3: } 62.8 \\
\text { D4: } 40.5\end{array}$ & ASEX & $\begin{array}{l}\text { Overall attrition } \\
\text { rate: } \\
13.3 \% \\
\text { ITT analysis: } \\
\text { Yes } \\
\text { Study Quality: } \\
\text { Fair }\end{array}$ \\
\hline
\end{tabular}


Sexual Dysfunction associated with Second-Generation Antidepressants in Patients with Major Depressive Disorder - Results from a Systematic Review with Network Meta-Analysis. Drug Safety; U. Reichenpfader, G. Gartlehner, L.C. Morgan, A. Greenblatt, B. Nussbaumer, R. A. Hansen, M. Van Noord, L. Lux, and B. N. Gaynes. Corresponding author: U. Reichenpfader, Department for Evidence-based Medicine and Clinical Epidemiology, Krems, Austria; Department of Medical and Health Sciences - Division of Community Medicine, Linköping University, Sweden; ureichenpfader@hotmail.com

\begin{tabular}{|c|c|c|c|c|c|c|}
\hline $\begin{array}{c}\text { Study } \\
\text { Characteristics }\end{array}$ & $\begin{array}{l}\text { Research Objective } \\
\text { Study Design }\end{array}$ & Inclusion/Exclusion & $\begin{array}{c}\text { Baseline } \\
\text { Characteristics }\end{array}$ & $\begin{array}{l}\text { Sexual Dysfunction } \\
\text { (SD) Outcomes/ } \\
\text { Adverse Events } \\
\end{array}$ & $\begin{array}{l}\text { Method used to } \\
\text { determine SD }\end{array}$ & $\begin{array}{c}\text { Analysis and Study } \\
\text { Quality (Risk of } \\
\text { Bias) }\end{array}$ \\
\hline $\begin{array}{l}\text { Author: } \\
\text { Duenas et al., } \\
2011 \text { [22] } \\
\text { Country and } \\
\text { setting: } \\
\text { Multicenter, } \\
\text { multiple } \\
\text { countries, } \\
\text { setting } \\
\text { Funding: } \\
\text { Eli Lilly and Co., } \\
\text { Lilly Research } \\
\text { Laboratories }\end{array}$ & 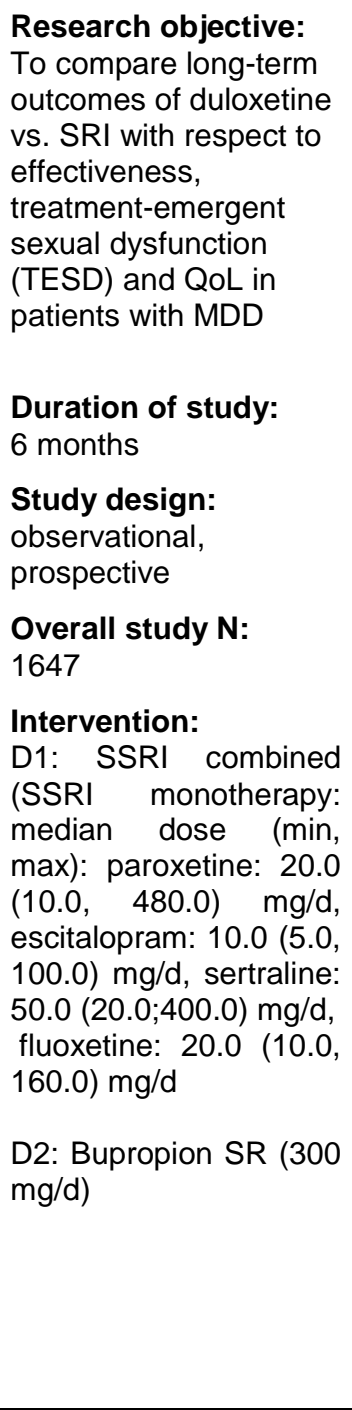 & $\begin{array}{l}\text { Inclusion criteria: } \\
\text { - Aged at least } 18 \\
\text { years } \\
\text { - sexually active } \\
\text { during } 2 \text { weeks prior } \\
\text { to study entry } \\
\text { without sexual } \\
\text { dysfunction } \\
\text { - Diagnosed with } \\
\text { MDD according to } \\
\text { DSM-IV or ICD-10 } \\
\text { with a CGI-S more } \\
\text { than } 4 \\
\text { Exclusion criteria: } \\
\text { - Concomitant } \\
\text { psychotheraputic or } \\
\text { psychotropic } \\
\text { medications } \\
\text { - any antidepresant } \\
\text { medication prior } 1 \\
\text { week to study } \\
\text { (Fluoxetine: } 1 \\
\text { month) } \\
\text { any current } \\
\text { psychiatric disorder } \\
\text { other than MDD as } \\
\text { defined in DSM-IV, } \\
\text { current or past } \\
\text { history of a manic or } \\
\text { hypomanic episode, } \\
\text { schizophrenia or } \\
\text { any other psychotic } \\
\text { disorder, mental } \\
\text { retardation, organic } \\
\text { mental disorders or } \\
\text { mental disorders } \\
\text { due to a general } \\
\text { medical condition, }\end{array}$ & $\begin{array}{l}\text { Groups similar at } \\
\text { baseline: } \\
\text { partially not balanced } \\
\text { Mean age (yrs): } \\
\text { D1: } 37.7 \\
\text { D2: } 38.2 \\
\text { Sex (\% female): } \\
\text { D1: } 58.3 \% \\
\text { D2: } 51.8 \% \\
\text { Race (\% white): } \\
\text { D1: } 18.1 \\
\text { D2: } 23.7 \\
\text { Baseline CGI-S score } \\
\text { (mean) } \\
\text { D1: } 4.5 \\
\text { D2: } 4.5 \\
\text { Mean MADRS score } \\
\text { at baseline: } \\
\text { D1: } 30.3 \\
\text { D2: } 30.4\end{array}$ & $\begin{array}{l}\text { TESD, } 6 \text { month } \\
\text { D1: physician } \\
\text { reported) } 28.7 \% \text { in } \\
\text { SSRI group ( } \mathrm{n}=509) \text {; } \\
\text { patient-reported } \\
\text { (TESD 6; } A S E X) \text { ): } \\
\text { approx. } 36 \% \text { (data } \\
\text { only shown in graph } \\
\text { D2: TESD } 6 \\
\text { (physician reported) } \\
23.4 \% \\
\text { \{OR 0.77; duloxetine } \\
\text { vs. SSRI } \\
\text { monotherapy) of } \\
\text { TESD } 6 \text { (95\% CI: } \\
\text { 0.57; } 1.04 \text { ); } \mathrm{P}=0.087 \text {; } \\
\text { patient-reported: } \\
\text { approx. } 16 \% \text { (data } \\
\text { only shown in graph) }\end{array}$ & $\begin{array}{l}\text { ASEX (physician- and } \\
\text { patient-assessed) }\end{array}$ & $\begin{array}{l}\text { Overall attrition } \\
\text { rate: } \\
23 \% \\
\text { ITT analysis: } \\
\text { NA } \\
\text { Risk of Bias: } \\
\text { Moderate }\end{array}$ \\
\hline
\end{tabular}


Sexual Dysfunction associated with Second-Generation Antidepressants in Patients with Major Depressive Disorder - Results from a Systematic Review with Network Meta-Analysis. Drug Safety; U. Reichenpfader, G. Gartlehner, L.C. Morgan, A. Greenblatt, B. Nussbaumer, R. A. Hansen, M. Van Noord, L. Lux, and B. N. Gaynes. Corresponding author: U. Reichenpfader, Department for Evidence-based Medicine and Clinical Epidemiology, Krems, Austria; Department of Medical and Health Sciences - Division of Community Medicine,

Linköping University, Sweden; ureichenpfader@hotmail.com 
Sexual Dysfunction associated with Second-Generation Antidepressants in Patients with Major Depressive Disorder - Results from a Systematic Review with Network Meta-Analysis Drug Safety; U. Reichenpfader, G. Gartlehner, L.C. Morgan, A. Greenblatt, B. Nussbaumer, R. A. Hansen, M. Van Noord, L. Lux, and B. N. Gaynes. Corresponding author: U. Reichenpfader, Department for Evidence-based Medicine and Clinical Epidemiology, Krems, Austria; Department of Medical and Health Sciences - Division of Community Medicine, Linköping University, Sweden; ureichenpfader@hotmail.com

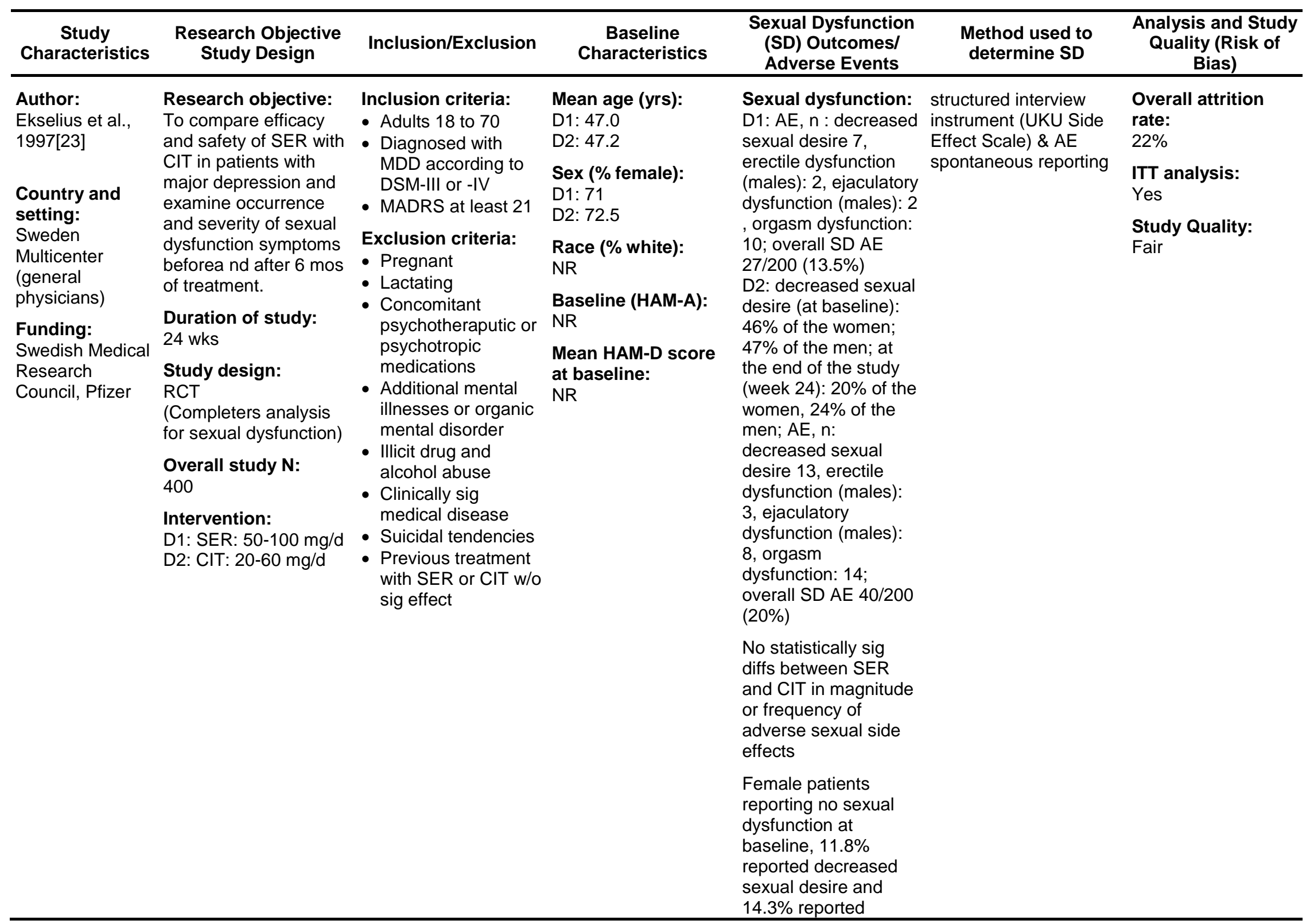


Sexual Dysfunction associated with Second-Generation Antidepressants in Patients with Major Depressive Disorder - Results from a Systematic Review with Network Meta-Analysis. Drug Safety; U. Reichenpfader, G. Gartlehner, L.C. Morgan, A. Greenblatt, B. Nussbaumer, R. A. Hansen, M. Van Noord, L. Lux, and B. N. Gaynes. Corresponding author: U. Reichenpfader, Department for Evidence-based Medicine and Clinical Epidemiology, Krems, Austria; Department of Medical and Health Sciences - Division of Community Medicine, Linköping University, Sweden; ureichenpfader@hotmail.com

\begin{tabular}{|c|c|c|c|c|c|c|}
\hline $\begin{array}{c}\text { Study } \\
\text { Characteristics }\end{array}$ & $\begin{array}{l}\text { Research Objective } \\
\text { Study Design }\end{array}$ & Inclusion/Exclusion & $\begin{array}{c}\text { Baseline } \\
\text { Characteristics }\end{array}$ & $\begin{array}{c}\text { Sexual Dysfunction } \\
\text { (SD) Outcomes/ } \\
\text { Adverse Events }\end{array}$ & $\begin{array}{l}\text { Method used to } \\
\text { determine SD }\end{array}$ & $\begin{array}{c}\text { Analysis and Study } \\
\text { Quality (Risk of } \\
\text { Bias) }\end{array}$ \\
\hline & & & & $\begin{array}{l}\text { orgasmic dysfunction } \\
\text { Male patients } \\
\text { reporting no sexual } \\
\text { dysfunction at } \\
\text { baseline, } 16.7 \% \\
\text { reported decreased } \\
\text { sexual desire, } 18.9 \% \\
\text { reported orgasmic } \\
\text { dysfunction, } 25 \% \\
\text { experienced } \\
\text { ejaculatory } \\
\text { dysfunction }\end{array}$ & & \\
\hline & & & & $\begin{array}{l}\text { Overall adverse } \\
\text { events: } \\
\text { D1: } 90 \\
\text { D2: } 85.5\end{array}$ & & \\
\hline
\end{tabular}


Sexual Dysfunction associated with Second-Generation Antidepressants in Patients with Major Depressive Disorder - Results from a Systematic Review with Network Meta-Analysis. Drug Safety; U. Reichenpfader, G. Gartlehner, L.C. Morgan, A. Greenblatt, B. Nussbaumer, R. A. Hansen, M. Van Noord, L. Lux, and B. N. Gaynes. Corresponding author: U. Reichenpfader, Department for Evidence-based Medicine and Clinical Epidemiology, Krems, Austria; Department of Medical and Health Sciences - Division of Community Medicine, Linköping University, Sweden; ureichenpfader@hotmail.com

\begin{tabular}{|c|c|c|c|c|c|c|}
\hline $\begin{array}{c}\text { Study } \\
\text { Characteristics }\end{array}$ & $\begin{array}{l}\text { Research Objective } \\
\text { Study Design }\end{array}$ & Inclusion/Exclusion & $\begin{array}{c}\text { Baseline } \\
\text { Characteristics }\end{array}$ & $\begin{array}{l}\text { Sexual Dysfunction } \\
\text { (SD) Outcomes/ } \\
\text { Adverse Events } \\
\end{array}$ & $\begin{array}{l}\text { Method used to } \\
\text { determine SD }\end{array}$ & $\begin{array}{c}\text { Analysis and Study } \\
\text { Quality (Risk of } \\
\text { Bias) } \\
\end{array}$ \\
\hline \multirow{3}{*}{$\begin{array}{l}\text { Author: } \\
\text { Fava et al., } \\
\text { 1998[24] } \\
\text { Country and } \\
\text { setting: } \\
\text { United States } \\
\text { Multicenter } \\
\text { (5 sites) }\end{array}$} & $\begin{array}{l}\text { Research objective: } \\
\text { Efficacy and tolerability } \\
\text { of PAR and FLUOX }\end{array}$ & \multirow{3}{*}{$\begin{array}{l}\text { Inclusion criteria: } \\
\text { - Minimum HAM-D } \\
\text { score of } 18 \\
\text { - Raskin Depression } \\
\text { score of > } 8 \text { (and } \\
\text { larger in value than } \\
\text { Covi anxiety scale) }\end{array}$} & \multirow{2}{*}{$\begin{array}{l}\text { Mean age (yrs): } \\
\text { D1: } 41.3 \\
\text { D2: } 41.3 \\
\text { D3: } 41.3\end{array}$} & \multirow{3}{*}{$\begin{array}{l}\text { Sexual dysfunction } \\
\text { (\%): } \\
\text { D1: } 25 \\
\text { D2: } 7 \\
\text { D3: } 0\end{array}$} & \multirow[t]{6}{*}{ spontaneously reported } & $\begin{array}{l}\text { Overall attrition } \\
\text { rate: } \\
28 \%\end{array}$ \\
\hline & $\begin{array}{l}\text { Duration of study: } \\
12 \text { wks }\end{array}$ & & & & & $\begin{array}{l}\text { ITT analysis: } \\
\text { Yes }\end{array}$ \\
\hline & $\begin{array}{l}\text { Study design: } \\
\text { RCT }\end{array}$ & & $\begin{array}{l}\text { Sex (\% female): } \\
\text { D1: } 50 \\
\text { D2: } 50 \\
\text { D3: } 50\end{array}$ & & & $\begin{array}{l}\text { Study Quality: } \\
\text { Fair }\end{array}$ \\
\hline \multirow{3}{*}{$\begin{array}{l}\text { Funding: } \\
\text { SmithKline, } \\
\text { Beecham }\end{array}$} & $\begin{array}{l}\text { Overall study N: } \\
128\end{array}$ & \multirow{3}{*}{$\begin{array}{l}\text { Exclusion criteria: } \\
\text { - Pregnant } \\
\text { - Lactating } \\
\text { - Concomitant } \\
\text { psychotheraputic or } \\
\text { psychotropic } \\
\text { medications } \\
\text { - Additional mental } \\
\text { illnesses or organic } \\
\text { mental disorder } \\
\text { - Illicit drug and } \\
\text { alcohol abuse } \\
\text { - ECT within last } 3 \\
\text { mos } \\
\text { - Suicidal tendencies }\end{array}$} & $\begin{array}{l}\text { Race (\% white): } \\
\text { NR }\end{array}$ & & & \\
\hline & $\begin{array}{l}\text { Intervention: } \\
\text { D1: PAR: } 20-50 \mathrm{mg} / \mathrm{d} \\
\quad \text { (initial dosage of }\end{array}$ & & $\begin{array}{l}\text { Baseline (HAM-A): } \\
\text { NR }\end{array}$ & & & \\
\hline & $\begin{array}{l}20 \mathrm{mg} / \mathrm{d} \text { could be } \\
\text { increased wkly by } \\
10 \mathrm{mg} / \mathrm{d} \text { up to } 50 \\
\mathrm{mg} / \mathrm{d} \text { ) } \\
\text { D2: FLUOX: } 20-80 \\
\mathrm{mg} / \mathrm{d} \text { (initial } \\
\text { dosage of } 20 \mathrm{mg} / \mathrm{d} \\
\text { could be increased } \\
\text { wkly by } 20 \mathrm{mg} / \mathrm{d} \text { up } \\
\text { to } 80 \mathrm{mg} / \mathrm{d} \text { ) } \\
\text { D3: PBO }\end{array}$ & & $\begin{array}{l}\text { Mean HAM-D score } \\
\text { at baseline: } \\
\text { D1: } 23.1(3.4) \\
\text { D2: } 23.9(3.8) \\
\text { D3: } 23.7(12.2)\end{array}$ & & & \\
\hline
\end{tabular}


Sexual Dysfunction associated with Second-Generation Antidepressants in Patients with Major Depressive Disorder - Results from a Systematic Review with Network Meta-Analysis. Drug Safety; U. Reichenpfader, G. Gartlehner, L.C. Morgan, A. Greenblatt, B. Nussbaumer, R. A. Hansen, M. Van Noord, L. Lux, and B. N. Gaynes. Corresponding author: U. Reichenpfader, Department for Evidence-based Medicine and Clinical Epidemiology, Krems, Austria; Department of Medical and Health Sciences - Division of Community Medicine, Linköping University, Sweden; ureichenpfader@hotmail.com

\begin{tabular}{|c|c|c|c|c|c|c|}
\hline $\begin{array}{c}\text { Study } \\
\text { Characteristics }\end{array}$ & $\begin{array}{l}\text { Research Objective } \\
\text { Study Design }\end{array}$ & Inclusion/Exclusion & $\begin{array}{c}\text { Baseline } \\
\text { Characteristics }\end{array}$ & $\begin{array}{c}\text { Sexual Dysfunction } \\
\text { (SD) Outcomes/ } \\
\text { Adverse Events }\end{array}$ & $\begin{array}{l}\text { Method used to } \\
\text { determine SD }\end{array}$ & $\begin{array}{c}\text { Analysis and Study } \\
\text { Quality (Risk of } \\
\text { Bias) }\end{array}$ \\
\hline $\begin{array}{l}\text { Author: } \\
\text { Fava et al, } 2002 \\
\text { [25] } \\
\text { Country and } \\
\text { setting: } \\
\text { USA, } \\
\text { Multicenter, } \\
\text { psychiatry } \\
\text { Funding: } \\
\text { Lilly Research } \\
\text { Laboratories, Eli } \\
\text { Lilly and } \\
\text { Company, US }\end{array}$ & $\begin{array}{l}\text { Research objective: } \\
\text { To analyze } \\
\text { discontinuation- } \\
\text { emergent adverse } \\
\text { events comparing } \\
\text { fluoxetine, sertraline, } \\
\text { and paroxetine in } \\
\text { patients with MDD and } \\
\text { to test whether the } \\
\text { presence of insomnia } \\
\text { disorder is predictive of } \\
\text { differential } \\
\text { responsiveness or } \\
\text { tolerability across } \\
\text { SSRIs } \\
\text { Duration of study: } \\
\text { 10-16 wks } \\
\text { Study design: } \\
\text { RCT, double-blind } \\
\text { Overall study N: } \\
\text { 284 } \\
\text { Intervention: } \\
\text { D1: Fluoxetine, 20- } \\
60 \mathrm{mg} / \mathrm{d} \\
\text { D2: Paroxetine, 20mg/d } \\
\text { D3: Sertraline, } 50- \\
200 \mathrm{mg} / \mathrm{d}\end{array}$ & $\begin{array}{l}\text { Inclusion criteria: } \\
\text { - Aged at least } 18 \\
\text { years } \\
\text { - Diagnosed with } \\
\text { MDD according to } \\
\text { DSM-IV of at least } \\
\text { 4-weeks' duration } \\
\text { - Minimum baseline } \\
\text { score of more than } \\
\text { on the first } 17 \text { items } \\
\text { (HAM-D-17) of the } \\
\text { HAM-D-28. } \\
\text { Exclusion criteria: } \\
\text { - Pregnancy or } \\
\text { breatfeeding } \\
\text { - concomitant use of } \\
\text { any antidepressant } \\
\text { (other than study } \\
\text { drugs), anxiolytic, } \\
\text { other psychotropic } \\
\text { medication within } 7 \\
\text { days before study } \\
\text { entry, with the } \\
\text { exception of chloral } \\
\text { hydrate; use of } \\
\text { MAOls within } 2 \\
\text { weeks of active } \\
\text { therapy or } \\
\text { anticipated need to } \\
\text { use an MAOI within } \\
5 \text { weeks of } \\
\text { discontinuing the } \\
\text { study } \\
\text { - serious suicidal risk; } \\
\text { - serious comorbid } \\
\text { illness that was not } \\
\text { stabilized; } \\
\text { - presence of a } \\
\text { seizure disorder }\end{array}$ & $\begin{array}{l}\text { Groups similar at } \\
\text { baseline: } \\
\text { yes } \\
\text { Mean age (yrs): } \\
\text { D1: } 42.1 \\
\text { D2: } 42.5 \\
\text { D3: } 44.0 \\
\text { Sex (\% female): } \\
\text { D1: } 63.0 \\
\text { D2: } 58.3 \\
\text { D3: } 57.3 \\
\text { Race (\% white): } \\
\text { NR } \\
\text { Mean (HAM-D-17) } \\
\text { score at baseline: } \\
\text { D1: } 20.5 \\
\text { D2: } 20.6 \\
\text { D3: } 21.0\end{array}$ & $\begin{array}{l}\text { D1: } 0 \\
\text { D2: } 20 \% \text { (abnormal } \\
\text { ejaculation) } \\
\text { D3: } 0\end{array}$ & open-ended question & $\begin{array}{l}\text { Overall attrition } \\
\text { rate: } \\
29.6 \% \\
\text { ITT analysis: } \\
\text { modified intent-to- } \\
\text { treat set at least one } \\
\text { post-baseline } \\
\text { visit at which } \\
\text { appropriate } \\
\text { measurements were } \\
\text { taken } \\
\text { Study Quality: } \\
\text { fair }\end{array}$ \\
\hline
\end{tabular}


Sexual Dysfunction associated with Second-Generation Antidepressants in Patients with Major Depressive Disorder - Results from a Systematic Review with Network Meta-Analysis. Drug Safety; U. Reichenpfader, G. Gartlehner, L.C. Morgan, A. Greenblatt, B. Nussbaumer, R. A. Hansen, M. Van Noord, L. Lux, and B. N. Gaynes. Corresponding author: U. Reichenpfader, Department for Evidence-based Medicine and Clinical Epidemiology, Krems, Austria; Department of Medical and Health Sciences - Division of Community Medicine, Linköping University, Sweden; ureichenpfader@hotmail.com

\begin{tabular}{|c|}
\hline $\begin{array}{l}\text { with a seizure } \\
\text { occurring within the } \\
\text { past year; } \\
\text { current or past } \\
\text { history of organic } \\
\text { mental disorder, } \\
\text { substance-use } \\
\text { disorder, } \\
\text { schizophrenia, } \\
\text { delusional disorder, } \\
\text { psychotic disorders } \\
\text { not elsewhere } \\
\text { classified, bipolar } \\
\text { disorder, and } \\
\text { antisocial } \\
\text { personality disorder; } \\
\text { mood-congruent or } \\
\text { mood-incongruent }\end{array}$ \\
\hline
\end{tabular}


Sexual Dysfunction associated with Second-Generation Antidepressants in Patients with Major Depressive Disorder - Results from a Systematic Review with Network Meta-Analysis. Drug Safety; U. Reichenpfader, G. Gartlehner, L.C. Morgan, A. Greenblatt, B. Nussbaumer, R. A. Hansen, M. Van Noord, L. Lux, and B. N. Gaynes. Corresponding author: U. Reichenpfader, Department for Evidence-based Medicine and Clinical Epidemiology, Krems, Austria; Department of Medical and Health Sciences - Division of Community Medicine, Linköping University, Sweden; ureichenpfader@hotmail.com

\begin{tabular}{|c|c|c|c|c|c|c|}
\hline $\begin{array}{c}\text { Study } \\
\text { Characteristics }\end{array}$ & $\begin{array}{l}\text { Research Objective } \\
\text { Study Design }\end{array}$ & Inclusion/Exclusion & $\begin{array}{c}\text { Baseline } \\
\text { Characteristics }\end{array}$ & $\begin{array}{l}\text { Sexual Dysfunction } \\
\text { (SD) Outcomes/ } \\
\text { Adverse Events } \\
\end{array}$ & $\begin{array}{l}\text { Method used to } \\
\text { determine SD }\end{array}$ & $\begin{array}{c}\text { Analysis and Study } \\
\text { Quality (Risk of } \\
\text { Bias) }\end{array}$ \\
\hline $\begin{array}{l}\text { Author: } \\
\text { Feiger et al., } \\
\text { 1996[26] } \\
\text { Country and } \\
\text { setting: } \\
\text { Europe } \\
\text { Multicenter (4) } \\
\text { Funding: } \\
\text { Bristol Myers } \\
\text { Squibb }\end{array}$ & $\begin{array}{l}\text { Research objective: } \\
\text { To compare safety and } \\
\text { efficacy of NEF with } \\
\text { SER in outpatients with } \\
\text { moderate to severe } \\
\text { depression } \\
\text { Duration of study: } \\
6 \text { wks } \\
\text { Study design: } \\
\text { RCT } \\
\text { Overall study N: } \\
\text { 160 } \\
\text { Intervention: } \\
\text { D1: NEF: } 100-600 \mathrm{mg} / \mathrm{d} \\
\text { D2: SER: } 50-200 \mathrm{mg} / \mathrm{d}\end{array}$ & $\begin{array}{l}\text { Inclusion criteria: } \\
\text { - Adults } 18 \text { or older } \\
\text { - Diagnosed with } \\
\text { MDD according to } \\
\text { DSM-III or -IV } \\
\text { - Minimum HAM-D } \\
\text { score of } 20 \\
\text { Exclusion criteria: } \\
\text { - Pregnant } \\
\text { - Lactating } \\
\text { - Illicit drug and } \\
\text { alcohol abuse } \\
\text { - Investigational drug } \\
\text { use } \\
\text { - Suicidal tendencies }\end{array}$ & $\begin{array}{l}\text { Mean age (yrs): } \\
\text { D1: } 43 \\
\text { D2: } 44.5 \\
\text { Sex (\% female): } \\
\text { D1: } 48 \\
\text { D2: } 55 \\
\text { Race (\% white): } \\
\text { D1: } 90 \\
\text { D2: } 79 \\
\text { Baseline (HAM-A): } \\
\text { NR } \\
\text { Mean HAM-D score } \\
\text { at baseline: } \\
\text { D1: } 23.5 \\
\text { D2: } 23.5\end{array}$ & $\begin{array}{l}\text { Difficulty with } \\
\text { ejaculation: } \\
\text { D1: no sig AE on } \\
\quad \text { sexual function } \\
\quad P<0.01 \\
\text { D2: had sig AEs on } \\
\quad \text { sexual function } \\
\text { D1: discontinuation } \\
\text { due to SD side } \\
\text { effects: } n=0 ; \\
\text { comparison of scores } \\
\text { from item responces } \\
\text { are shown an } \\
\text { differences calculated; } \\
\text { since data from } \\
\text { unvalidated } \\
\text { questionnaire only, no } \\
\text { estimation of SD } \\
\text { possible } \\
\text { D2: Erectile } \\
\text { dysfunction: } 7 / 45 \\
\text { (15.6\% of men) } \\
\text { Overall adverse } \\
\text { events: } \\
\text { D1: } 96 \\
\text { D2: } 95\end{array}$ & $\begin{array}{l}\text { unvalidated } \mathrm{SD} \\
\text { questionnaire, } \mathrm{AE} \\
\text { spontanceous reporting }\end{array}$ & $\begin{array}{l}\text { Overall attrition } \\
\text { rate: } \\
24.4 \% \\
\text { ITT analysis: } \\
\text { Yes } \\
\text { Study Quality: } \\
\text { Fair }\end{array}$ \\
\hline
\end{tabular}


Sexual Dysfunction associated with Second-Generation Antidepressants in Patients with Major Depressive Disorder - Results from a Systematic Review with Network Meta-Analysis Drug Safety; U. Reichenpfader, G. Gartlehner, L.C. Morgan, A. Greenblatt, B. Nussbaumer, R. A. Hansen, M. Van Noord, L. Lux, and B. N. Gaynes. Corresponding author: U. Reichenpfader, Department for Evidence-based Medicine and Clinical Epidemiology, Krems, Austria; Department of Medical and Health Sciences - Division of Community Medicine, Linköping University, Sweden; ureichenpfader@hotmail.com

\begin{tabular}{|c|c|c|c|c|c|c|}
\hline $\begin{array}{c}\text { Study } \\
\text { Characteristics }\end{array}$ & $\begin{array}{l}\text { Research Objective } \\
\text { Study Design }\end{array}$ & Inclusion/Exclusion & $\begin{array}{c}\text { Baseline } \\
\text { Characteristics }\end{array}$ & $\begin{array}{c}\text { Sexual Dysfunction } \\
\text { (SD) Outcomes/ } \\
\text { Adverse Events } \\
\end{array}$ & $\begin{array}{l}\text { Method used to } \\
\text { determine SD }\end{array}$ & $\begin{array}{c}\text { Analysis and Study } \\
\text { Quality (Risk of } \\
\text { Bias) }\end{array}$ \\
\hline $\begin{array}{l}\text { Author: } \\
\text { Feighner et al., } \\
\text { 1991[27] } \\
\text { Country and } \\
\text { setting: } \\
\text { United States } \\
\text { Multicenter (2 } \\
\text { sites) } \\
\text { Funding: } \\
\text { Burroughs } \\
\text { Wellcome Co }\end{array}$ & $\begin{array}{l}\text { Research objective: } \\
\text { Efficacy and safety of } \\
\text { BUP and FLUOX in } \\
\text { depressed outpatients } \\
\text { Duration of study: } \\
6 \text { wks } \\
\text { Study design: } \\
\text { RCT } \\
\text { Overall study N: } \\
\text { 123 } \\
\text { Intervention: } \\
\text { D1: BUP: } 225-450 \\
\text { mg/d (382) } \\
\text { D2: FLUOX: } 20-80 \\
\text { mg/d (38) }\end{array}$ & $\begin{array}{l}\text { Inclusion criteria: } \\
\text { - Adults } 18 \text { or older } \\
\text { - Diagnosed with } \\
\text { MDD according to } \\
\text { DSM-III or -IV } \\
\text { - Minimum HAM-D } \\
\text { score of } 20 \\
\text { Exclusion criteria: } \\
\text { - Pregnant } \\
\text { - Lactating } \\
\text { - Concomitant } \\
\text { psychotheraputic or } \\
\text { psychotropic } \\
\text { medications } \\
\text { - Illicit drug and } \\
\text { alcohol abuse } \\
\text { - Clinically sig } \\
\text { medical disease } \\
\text { - Suicidal tendencies }\end{array}$ & $\begin{array}{l}\text { Mean age (yrs): } \\
\text { D1: } 40.9 \\
\text { D2: } 42.9 \\
\text { Sex (\% female): } \\
\text { D1: } 62 \\
\text { D2: } 61 \\
\text { Race (\% white): } \\
\text { NR } \\
\text { Baseline (HAM-A): } \\
\text { NR } \\
\text { Mean HAM-D score } \\
\text { at baseline: } \\
\text { D1: } 25.3 \\
\text { D2: } 26.1\end{array}$ & $\begin{array}{l}\text { D1: } 0 \% \\
\text { D2: impotence/erectile } \\
\text { dysfunction } 4.7 \% \text {, } \\
\text { anorgasmia } 1.7 \% \text {, and } \\
\text { libido decrease } 1.7 \% \\
(P=N R)\end{array}$ & $\begin{array}{l}\text { standardized open } \\
\text { question to elicit } A E\end{array}$ & $\begin{array}{l}\text { Overall Attrition } \\
\text { rate: } \\
7.3 \% \\
\text { ITT Analysis: } \\
\text { Yes } \\
\text { Study Quality: } \\
\text { Fair }\end{array}$ \\
\hline
\end{tabular}


Sexual Dysfunction associated with Second-Generation Antidepressants in Patients with Major Depressive Disorder - Results from a Systematic Review with Network Meta-Analysis Drug Safety; U. Reichenpfader, G. Gartlehner, L.C. Morgan, A. Greenblatt, B. Nussbaumer, R. A. Hansen, M. Van Noord, L. Lux, and B. N. Gaynes. Corresponding author: U. Reichenpfader, Department for Evidence-based Medicine and Clinical Epidemiology, Krems, Austria; Department of Medical and Health Sciences - Division of Community Medicine, Linköping University, Sweden; ureichenpfader@hotmail.com

\begin{tabular}{|c|c|c|c|c|c|c|}
\hline $\begin{array}{c}\text { Study } \\
\text { Characteristics }\end{array}$ & $\begin{array}{l}\text { Research Objective } \\
\text { Study Design }\end{array}$ & Inclusion/Exclusion & $\begin{array}{c}\text { Baseline } \\
\text { Characteristics }\end{array}$ & $\begin{array}{c}\text { Sexual Dysfunction } \\
\text { (SD) Outcomes/ } \\
\text { Adverse Events }\end{array}$ & $\begin{array}{l}\text { Method used to } \\
\text { determine SD }\end{array}$ & $\begin{array}{c}\text { Analysis and Study } \\
\text { Quality (Risk of } \\
\text { Bias) }\end{array}$ \\
\hline $\begin{array}{l}\text { Author: } \\
\text { Ferguson et al., } \\
2001[28] \\
\text { Country and } \\
\text { setting: } \\
\text { United States } \\
\text { Multicenter (9 } \\
\text { sites) } \\
\text { Funding: } \\
\text { Bristol Myers } \\
\text { Squibb }\end{array}$ & $\begin{array}{l}\text { Research objective: } \\
\text { To compare effects of } \\
\text { NEF and SER on } \\
\text { reemergence rates of } \\
\text { sexual dysfunction in } \\
\text { depressed patients } \\
\text { who'd had sexual } \\
\text { dysfunction with } \\
\text { previous SER } \\
\text { treatment } \\
\text { Duration of study: } \\
8 \text { wks } \\
\text { Study design: } \\
\text { RCT } \\
\text { Overall study N: } \\
75 \\
\text { Intervention: } \\
\text { D1: NEF: } 200-400 \mathrm{mg} / \mathrm{d} \\
\text { D2: SER: } 50-100 \mathrm{mg} / \mathrm{d}\end{array}$ & $\begin{array}{l}\text { Inclusion criteria: } \\
\text { - Adults } 18 \text { to } 65 \\
\text { - Diagnosed with } \\
\text { MDD according to } \\
\text { DSM-III or -IV } \\
\text { - Receiving SER and } \\
\text { experiencing } \\
\text { attributable sexual } \\
\text { dysfunction } \\
\text { Exclusion criteria: } \\
\text { - Pregnant } \\
\text { - Lactating } \\
\text { - Concomitant } \\
\text { psychotheraputic or } \\
\text { psychotropic } \\
\text { medications } \\
\text { - Additional mental } \\
\text { illnesses or organic } \\
\text { mental disorder } \\
\text { - Illicit drug and } \\
\text { alcohol abuse } \\
\text { - Clinically sig } \\
\text { medical disease } \\
\text { - Investigational drug } \\
\text { use within last } 30 \\
\text { days }\end{array}$ & $\begin{array}{l}\text { Mean age (yrs): } \\
\text { D1: } 43.2 \\
\text { D2: } 44.8 \\
\text { Sex (\% female): } \\
\text { D1: } 46 \\
\text { D2: } 48 \\
\text { Race (\% white): } \\
\text { D1: } 95 \\
\text { D2: } 97 \\
\text { Baseline (HAM-A): } \\
\text { NR } \\
\text { Baseline HAM-D: } \\
\text { D1: } 11.5 \\
\text { D2: } 10.5\end{array}$ & $\begin{array}{l}\text { Sexual dysfunctional } \\
\text { (male ejaculation): } \\
\text { D1: } 26 \%(10 / 39 \\
\text { D2: } 76 \%(25 / 33) \\
\text { More SER treated } \\
\text { patients had } \\
\text { reemergence of } \\
\text { sexual dysfunction } \\
\text { than nefazadone- } \\
\text { treated }(76 \% \text { vs. } 26 \% \text {; } \\
P<0.001) \text {; } \\
\text { Overall adverse } \\
\text { events: } \\
\text { D1: } 100 \\
\text { D2: } 97\end{array}$ & $\begin{array}{l}\text { Physicians Rating of } \\
\text { Sexual Dysfunction } \\
\text { Dymptoms (PRSDS), } \\
\text { Rush-Presbytarian } \\
\text { Sexual Function } \\
\text { Inventory (R-SFI); AE } \\
\text { spontaneously reported }\end{array}$ & $\begin{array}{l}\text { Overall attrition } \\
\text { rate: } \\
32 \% \\
\text { ITT Analysis } \\
\text { Yes } \\
\text { Study Quality: } \\
\text { Fair }\end{array}$ \\
\hline
\end{tabular}


Sexual Dysfunction associated with Second-Generation Antidepressants in Patients with Major Depressive Disorder - Results from a Systematic Review with Network Meta-Analysis. Drug Safety; U. Reichenpfader, G. Gartlehner, L.C. Morgan, A. Greenblatt, B. Nussbaumer, R. A. Hansen, M. Van Noord, L. Lux, and B. N. Gaynes. Corresponding author: U. Reichenpfader, Department for Evidence-based Medicine and Clinical Epidemiology, Krems, Austria; Department of Medical and Health Sciences - Division of Community Medicine,

Linköping University, Sweden; ureichenpfader@hotmail.com

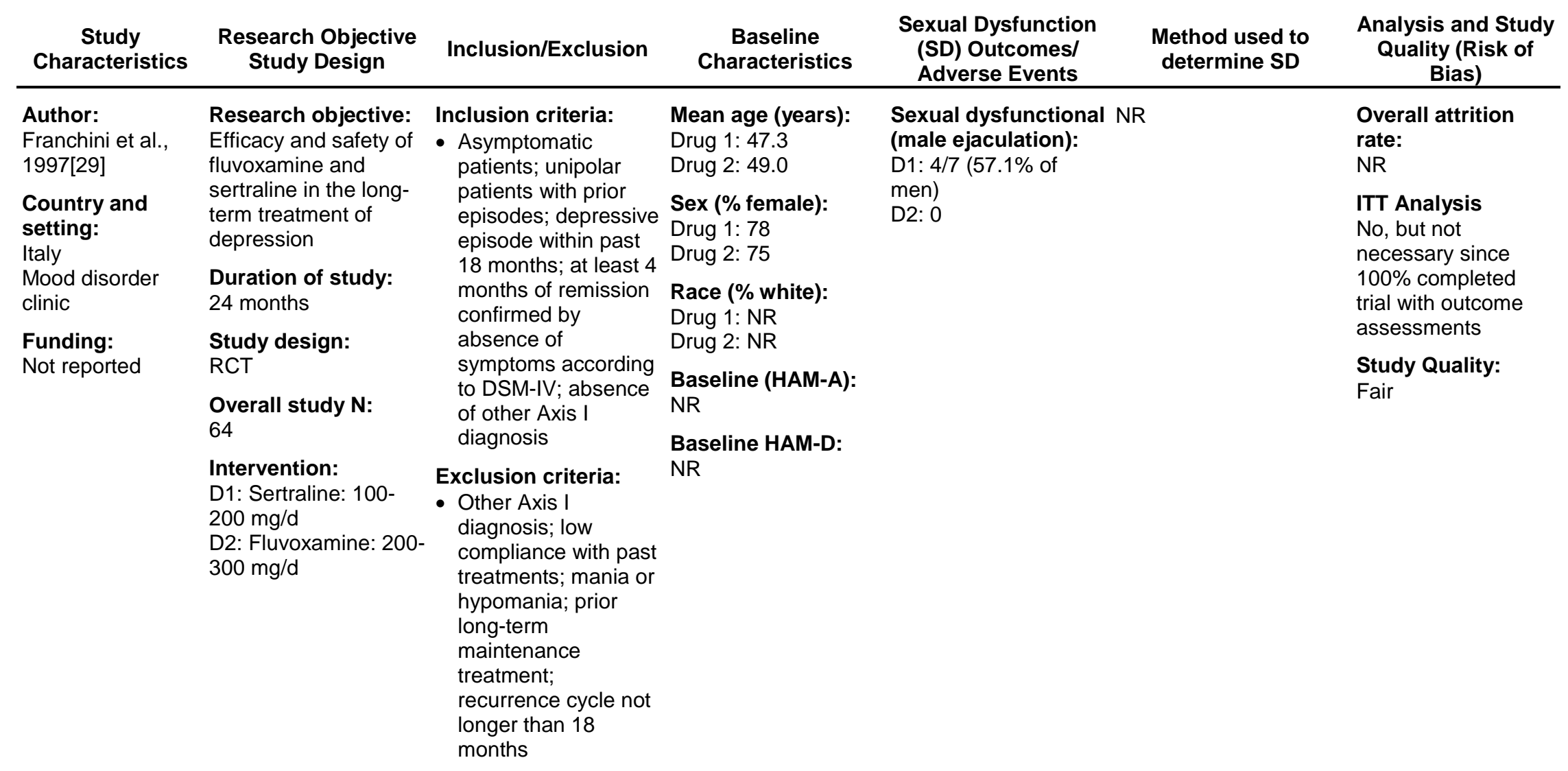


Sexual Dysfunction associated with Second-Generation Antidepressants in Patients with Major Depressive Disorder - Results from a Systematic Review with Network Meta-Analysis Drug Safety; U. Reichenpfader, G. Gartlehner, L.C. Morgan, A. Greenblatt, B. Nussbaumer, R. A. Hansen, M. Van Noord, L. Lux, and B. N. Gaynes. Corresponding author: U. Reichenpfader, Department for Evidence-based Medicine and Clinical Epidemiology, Krems, Austria; Department of Medical and Health Sciences - Division of Community Medicine, Linköping University, Sweden; ureichenpfader@hotmail.com

\begin{tabular}{|c|c|c|c|c|c|c|}
\hline $\begin{array}{c}\text { Study } \\
\text { Characteristics }\end{array}$ & $\begin{array}{l}\text { Research Objective } \\
\text { Study Design }\end{array}$ & Inclusion/Exclusion & $\begin{array}{c}\text { Baseline } \\
\text { Characteristics }\end{array}$ & $\begin{array}{l}\text { Sexual Dysfunction } \\
\text { (SD) Outcomes/ } \\
\text { Adverse Events } \\
\end{array}$ & $\begin{array}{l}\text { Method used to } \\
\text { determine SD }\end{array}$ & $\begin{array}{c}\text { Analysis and Study } \\
\text { Quality (Risk of } \\
\text { Bias) } \\
\end{array}$ \\
\hline $\begin{array}{l}\text { Author: } \\
\text { Gelenberg et al., } \\
\text { 2003[30] }\end{array}$ & \multirow{2}{*}{$\begin{array}{l}\text { Research objective: } \\
\text { Comparison of NEF } \\
\text { and PBO in prevention } \\
\text { of depression } \\
\text { recurrence }\end{array}$} & \multirow{3}{*}{$\begin{array}{l}\text { Inclusion criteria: } \\
\text { - Adults } 18 \text { to } 75 \\
\text { - Diagnosed with } \\
\text { MDD according to } \\
\text { DSM-III or -IV } \\
\text { - Minimum HAM-D } \\
\text { score of } 20\end{array}$} & $\begin{array}{l}\text { Mean age (yrs): } \\
\text { D1: } 44.4 \\
\text { D2: } 44.1 \\
\text { D3: } 44.0\end{array}$ & \multirow[t]{8}{*}{$\begin{array}{l}\text { SD } \\
\text { D1: } 2 / 78(2.6 \%) \\
\text { D2: } 3 / 87(3.4 \%)\end{array}$} & \multirow[t]{8}{*}{ spontaneously reported } & \multirow{8}{*}{$\begin{array}{l}\text { Overall attrition } \\
\text { rate: } \\
50.6 \% \\
\text { ITT Analysis } \\
\text { Yes } \\
\text { Study Quality: } \\
\text { Fair }\end{array}$} \\
\hline $\begin{array}{l}\text { Country and } \\
\text { setting: }\end{array}$ & & & \multirow{3}{*}{$\begin{array}{l}\text { Sex (\% female): } \\
\text { D1: } 69.7 \\
\text { D2: } 65.5 \\
\text { D3: } 67.5\end{array}$} & & & \\
\hline $\begin{array}{l}\text { United States } \\
\text { Multiclinic }\end{array}$ & $\begin{array}{l}\text { Duration of study: } \\
52 \text { wks }\end{array}$ & & & & & \\
\hline \multirow{5}{*}{$\begin{array}{l}\text { Funding: } \\
\text { Bristol-Myers- } \\
\text { Squibb }\end{array}$} & Study design: & \multirow{5}{*}{$\begin{array}{l}\text { Exclusion criteria: } \\
\text { - Pregnant } \\
\text { - Additional mental } \\
\text { illnesses or organic } \\
\text { mental disorder } \\
\text { - Illicit drug and } \\
\text { alcohol abuse } \\
\text { - Clinically sig } \\
\text { medical disease } \\
\text { - ECT within last } 3 \\
\text { mos } \\
\text { - Suicidial tendencies }\end{array}$} & & & & \\
\hline & \multirow{3}{*}{$\begin{array}{l}\text { Overall study } \mathbf{N} \text { : } \\
165 \text { for maintenance } \\
\text { phase }\end{array}$} & & Race (\% white): & & & \\
\hline & & & Uverall. 90.5 & & & \\
\hline & & & $\begin{array}{l}\text { Baseline HAM-A: } \\
\text { NR }\end{array}$ & & & \\
\hline & $\begin{array}{l}\text { Intervention: } \\
\text { D1: NEF: 300-600 mg/d } \\
\quad(495.2) \\
\text { D2: PBO } \\
\text { D3: Overall }\end{array}$ & & $\begin{array}{l}\text { Baseline HAM-D: } \\
\text { NR }\end{array}$ & & & \\
\hline
\end{tabular}


Sexual Dysfunction associated with Second-Generation Antidepressants in Patients with Major Depressive Disorder - Results from a Systematic Review with Network Meta-Analysis. Drug Safety; U. Reichenpfader, G. Gartlehner, L.C. Morgan, A. Greenblatt, B. Nussbaumer, R. A. Hansen, M. Van Noord, L. Lux, and B. N. Gaynes. Corresponding author: U. Reichenpfader, Department for Evidence-based Medicine and Clinical Epidemiology, Krems, Austria; Department of Medical and Health Sciences - Division of Community Medicine, Linköping University, Sweden; ureichenpfader@hotmail.com

\begin{tabular}{|c|c|c|c|c|c|c|}
\hline $\begin{array}{c}\text { Study } \\
\text { Characteristics }\end{array}$ & $\begin{array}{l}\text { Research Objective } \\
\text { Study Design }\end{array}$ & Inclusion/Exclusion & $\begin{array}{c}\text { Baseline } \\
\text { Characteristics }\end{array}$ & $\begin{array}{c}\text { Sexual Dysfunction } \\
\text { (SD) Outcomes/ } \\
\text { Adverse Events } \\
\end{array}$ & $\begin{array}{l}\text { Method used to } \\
\text { determine SD }\end{array}$ & $\begin{array}{c}\text { Analysis and Study } \\
\text { Quality (Risk of } \\
\text { Bias) }\end{array}$ \\
\hline $\begin{array}{l}\text { Author: } \\
\text { Gilaberte et al., } \\
2001[31] \\
\text { Country and } \\
\text { setting: } \\
\text { Spain; } \\
\text { multicenter (10) } \\
\text { Funding: } \\
\text { Eli Lilly and Co }\end{array}$ & $\begin{array}{l}\text { Research objective: } \\
\text { To evaluate efficacy } \\
\text { and safety of FLUOX } \\
\text { compared to PBO in } \\
\text { maintenance treatment } \\
\text { of recurrent unipolar } \\
\text { depression } \\
\text { Duration of study: } \\
1 \text { yr for maintenance } \\
\text { (2 yrs total) } \\
\text { Study design: } \\
\text { RCT } \\
\text { Overall study N: } \\
\text { 140 (double-blind } \\
\text { maintenance phase) } \\
\text { Intervention: } \\
\text { D1: FLUOX: } 20-40 \\
\quad \text { mg/d } \\
\text { D2: PBO }\end{array}$ & $\begin{array}{l}\text { Inclusion criteria: } \\
\text { - Adults } 18 \text { to } 65 \\
\text { - Diagnosed with } \\
\text { MDD according to } \\
\text { DSM-III or -IV } \\
\text { - Minimum HAM-D } \\
\text { score of } 18 \\
\text { - At least one prior } \\
\text { depressive episode } \\
\text { in last } 5 \text { yrs } \\
\text { - CGI-S score at least } \\
4 \text { in index episode } \\
\text { Exclusion criteria: } \\
\text { - Pregnant } \\
\text { - Lactating } \\
\text { - Additional mental } \\
\text { illnesses or organic } \\
\text { mental disorder } \\
\text { - Illicit drug and } \\
\text { alcohol abuse } \\
\text { - Suicidial tendencies } \\
\text { - Previous resistance } \\
\text { to pharmacologic } \\
\text { treatment }\end{array}$ & $\begin{array}{l}\text { Mean age (yrs): } \\
\text { D1: } 44.4 \\
\text { D2: } 43.8 \\
\text { Sex (\% female): } \\
\text { D1: } 78.6 \\
\text { D2: } 78.6 \\
\text { Race (\% white): } \\
\text { NR } \\
\text { Baseline HAM-A: } \\
\text { NR } \\
\text { Baseline HAM-D: } \\
\text { D1: } 2.8 \text { (2.0) } \\
\text { D2: } 3.1 \text { (2.7) }\end{array}$ & $\begin{array}{l}\text { D1: } 9 / 70(12.9 \%) \\
\text { D2: } 10 / 70: 14.3 \% \\
\text { Overall adverse } \\
\text { events: } \\
\text { D1: } 62.9 \\
\text { D2: } 68.6\end{array}$ & nonprobing inquiry & $\begin{array}{l}\text { Overall attrition } \\
\text { rate: } \\
44.3 \% \\
\text { ITT Analysis } \\
\text { Yes } \\
\text { Study Quality: } \\
\text { Fair }\end{array}$ \\
\hline
\end{tabular}


Sexual Dysfunction associated with Second-Generation Antidepressants in Patients with Major Depressive Disorder - Results from a Systematic Review with Network Meta-Analysis. Drug Safety; U. Reichenpfader, G. Gartlehner, L.C. Morgan, A. Greenblatt, B. Nussbaumer, R. A. Hansen, M. Van Noord, L. Lux, and B. N. Gaynes. Corresponding author: U. Reichenpfader, Department for Evidence-based Medicine and Clinical Epidemiology, Krems, Austria; Department of Medical and Health Sciences - Division of Community Medicine, Linköping University, Sweden; ureichenpfader@hotmail.com

\begin{tabular}{|c|c|c|c|c|c|}
\hline $\begin{array}{c}\text { Study } \\
\text { Characteristics }\end{array}$ & $\begin{array}{l}\text { Research Objective } \\
\text { Study Design }\end{array}$ & $\begin{array}{l}\text { Inclusion/Exclusion } \\
\text { Outcome Measures }\end{array}$ & $\begin{array}{c}\text { Population } \\
\text { Characteristics }\end{array}$ & $\begin{array}{c}\text { Sexual Dysfunction (SD) } \\
\text { Outcomes/ Adverse } \\
\text { Events }\end{array}$ & $\begin{array}{l}\text { Method used to } \\
\text { determine SD }\end{array}$ \\
\hline $\begin{array}{l}\text { Author, Year } \\
\text { Golden et al., } \\
\text { 2002[32] } \\
\text { Country and } \\
\text { Setting } \\
\text { USA and Canada, } \\
\text { multicenter } \\
\text { Funding } \\
\text { GlaxoSmithKline } \\
\text { Study Quality: } \\
\text { Fair } \\
\text { (high attrition) } \\
\\
\text { Is adherence } \\
\text { reported? } \\
\text { NR } \\
\text { Rate of } \\
\text { adherence or } \\
\text { compliance } \\
\text { NR }\end{array}$ & $\begin{array}{l}\text { Research objective } \\
\text { To determine antidepressant } \\
\text { efficacy and tolerability of } \\
\text { PAR CR and PAR IR in adult } \\
\text { patients with MDD. } \\
\text { Drugs, Doses, and Range } \\
\text { - PAR (10-60 mg } 1 \mathrm{x} \text { daily): } \\
20-50 \mathrm{mg} / \mathrm{day} \text { (low to high) } \\
\text { - PAR CR (12.5-75 mg } 1 \mathrm{x} \\
\text { daily): } 25-62.5 \mathrm{mg} / \mathrm{day} \\
\text { (low to high) } \\
\text { Fixed dose } \\
\text { No } \\
\text { Flexible dose } \\
\text { Yes } \\
\text { Dosages equivalent } \\
\text { Yes } \\
\text { Study design } \\
\text { RCT } \\
\text { Duration } \\
12 \text { weeks } \\
\text { Type of depression } \\
\text { MDD }\end{array}$ & $\begin{array}{l}\text { Inclusion criteria: } \\
\text { - Adults (age range): 18- } \\
65 \\
\text { - Diagnosed with MDD } \\
\text { according to DSM-III or } \\
\text {-IV } \\
\text { - HAM-D: } 20 \text { or more } \\
\text { (and did not decrease } \\
\text { by more than } 25 \% \\
\text { between screening and } \\
\text { baseline } \\
\text { Exclusion criteria: } \\
\text { - Concomitant } \\
\text { psychotheraputic or } \\
\text { psychotropic } \\
\text { medications: treatment } \\
\text { with monoamine } \\
\text { oxidase inhibitor, } \\
\text { benzodiazepine, or } \\
\text { other psychoactive } \\
\text { agent (excluding chloral } \\
\text { hydrate) } \\
\text { - Additional mental } \\
\text { illnesses or organic } \\
\text { mental disorder not } \\
\text { related to depression } \\
\text { (e.g., schizophrenia, } \\
\text { bipolar) } \\
\text { - Illicit drug and alcohol } \\
\text { abuse within } 6 \text { months } \\
\text { of screening } \\
\text { - ECT within last: } 3 \\
\text { months } \\
\text { - Suicidal tendencies } \\
\text { (acute or other) } \\
\text { - History of brief } \\
\text { depressive episodes } \\
\text { (s8 weeks) } \\
\text { - Homicidal risk }\end{array}$ & $\begin{array}{l}\text { Groups similar at } \\
\text { baseline } \\
\text { Yes } \\
\mathbf{n}= \\
\text { D1: } 212 \\
\text { D2: } 217 \\
\text { D3: } 211 \\
\text { Mean age, years } \\
\text { D1: } 40.7 \\
\text { D2: } 39.9 \\
\text { D3: } 39.7 \\
\text { Sex, \% female } \\
\text { D1: } 63.2 \\
\text { D2: } 69.1 \\
\text { D3: } 63.0 \\
\text { Race, \% white } \\
\text { D1: } 88.2 \\
\text { D2: } 86.6 \\
\text { D3: } 85.3 \\
\text { Baseline HAM-A } \\
\text { NR } \\
\text { Insomnia, \% } \\
\text { NR } \\
\text { Concomitant anergia, \% } \\
\text { NR } \\
\text { Experienced prior } \\
\text { depressive episodes, \% } \\
\text { NR } \\
\text { Comments: } \\
\text { Outpatients/lnpatients } \\
\text { Baseline mean HAM-A > } \\
\text { 25? } \\
\text { NR }\end{array}$ & $\begin{array}{l}\text { Sexual dysfunction: } \\
\text { Abnormal ejaculation: } \\
\text { D1: overall SD AE: } 24 / 212 \\
(11.3 \%) \\
\text { D2: at least } 5 \% \text { and } 2 x \text { the } \\
\text { rate of placebo- } \\
\text { group; } \\
\text { D3: } 1 \text { female, } 1 \text { male: total } \\
\text { SD } 2 / 211(0.095 \%)\end{array}$ & non-leading question \\
\hline
\end{tabular}


Sexual Dysfunction associated with Second-Generation Antidepressants in Patients with Major Depressive Disorder - Results from a Systematic Review with Network Meta-Analysis. Drug Safety; U. Reichenpfader, G. Gartlehner, L.C. Morgan, A. Greenblatt, B. Nussbaumer, R. A. Hansen, M. Van Noord, L. Lux, and B. N. Gaynes. Corresponding author: U. Reichenpfader, Department for Evidence-based Medicine and Clinical Epidemiology, Krems, Austria; Department of Medical and Health Sciences - Division of Community Medicine, Linköping University, Sweden; ureichenpfader@hotmail.com

\begin{tabular}{|c|c|c|c|c|c|}
\hline $\begin{array}{c}\text { Study } \\
\text { Characteristics }\end{array}$ & $\begin{array}{l}\text { Research Objective } \\
\text { Study Design }\end{array}$ & $\begin{array}{l}\text { Inclusion/Exclusion } \\
\text { Outcome Measures }\end{array}$ & $\begin{array}{c}\text { Population } \\
\text { Characteristics }\end{array}$ & $\begin{array}{c}\text { Sexual Dysfunction (SD) } \\
\text { Outcomes/ Adverse } \\
\text { Events }\end{array}$ & $\begin{array}{l}\text { Method used to } \\
\text { determine SD }\end{array}$ \\
\hline $\begin{array}{l}\text { to lack of } \\
\text { efficacy, \% } \\
\text { NR }\end{array}$ & & $\begin{array}{l}\text { - Currently taking PAR or } \\
\text { history of PAR } \\
\text { nonresponse or }\end{array}$ & $\begin{array}{l}\text { Mean age at baseline } \\
\text { Less than } 65 \text { years }\end{array}$ & & \\
\hline $\begin{array}{l}\text { Comments } \\
\text { Dropout rate of } \\
\text { patients with PAR } \\
\text { IR sign. higher } \\
\text { compared to PBO } \\
(P=0.0008)\end{array}$ & & $\begin{array}{l}\text { intolerability } \\
\text { Outcome measures } \\
\text { - HAM-D: 17-item total } \\
\text { score, depressed mood } \\
\text { (item 1), psychic } \\
\text { anxiety (item 10) }\end{array}$ & $\begin{array}{l}\text { Mean HAM-D at baseline } \\
\text { Greater than } 17 \\
\text { (moderate to severe) }\end{array}$ & & \\
\hline
\end{tabular}


Sexual Dysfunction associated with Second-Generation Antidepressants in Patients with Major Depressive Disorder - Results from a Systematic Review with Network Meta-Analysis Drug Safety; U. Reichenpfader, G. Gartlehner, L.C. Morgan, A. Greenblatt, B. Nussbaumer, R. A. Hansen, M. Van Noord, L. Lux, and B. N. Gaynes. Corresponding author: U. Reichenpfader, Department for Evidence-based Medicine and Clinical Epidemiology, Krems, Austria; Department of Medical and Health Sciences - Division of Community Medicine, Linköping University, Sweden; ureichenpfader@hotmail.com

\begin{tabular}{|c|c|c|c|c|c|c|}
\hline $\begin{array}{c}\text { Study } \\
\text { Characteristics }\end{array}$ & $\begin{array}{l}\text { Research Objective } \\
\text { Study Design }\end{array}$ & Inclusion/Exclusion & $\begin{array}{c}\text { Baseline } \\
\text { Characteristics }\end{array}$ & $\begin{array}{c}\text { Sexual Dysfunction } \\
\text { (SD) Outcomes/ } \\
\text { Adverse Events }\end{array}$ & $\begin{array}{l}\text { Method used to } \\
\text { determine SD }\end{array}$ & $\begin{array}{c}\text { Analysis and Study } \\
\text { Quality (Risk of } \\
\text { Bias) }\end{array}$ \\
\hline $\begin{array}{l}\text { Author: } \\
\text { Guelfi et al., } \\
2001[33] \\
\text { Country and } \\
\text { setting: } \\
\text { France, } \\
\text { Denmark, } \\
\text { Belgium, } \\
\text { Netherlands } \\
\text { Multicenter (33) } \\
\text { Funding: } \\
\text { N.V. Organon, } \\
\text { Oss, Netherlands }\end{array}$ & $\begin{array}{l}\text { Research objective: } \\
\text { To compare } \\
\text { antidepressant efficacy } \\
\text { and tolerability of MIR } \\
\text { and VEN in treatment } \\
\text { of hospitalized patients } \\
\text { with DSM-IV diagnosis } \\
\text { of severe depressive } \\
\text { episode with } \\
\text { melancholic features } \\
\text { Duration of study: } \\
8 \text { wks } \\
\text { Study design: } \\
\text { RCT } \\
\text { Overall study N: } \\
\text { 157 } \\
\text { Intervention: } \\
\text { D1: MIR: } 49.5 \text { mg } \\
\text { D2: VEN: } 255.0 \mathrm{mg}\end{array}$ & $\begin{array}{l}\text { Inclusion criteria: } \\
\text { - Adults } 18 \text { to } 65 \\
\text { - Diagnosed with } \\
\text { MDD according to } \\
\text { DSM-III or -IV } \\
\text { - Minimum HAM-D } \\
\text { score of } 25 \\
\text { - DSM-IV melancholic } \\
\text { features } \\
\text { Exclusion criteria: } \\
\text { - Pregnant } \\
\text { - Lactating } \\
\text { - Concomitant } \\
\text { psychotheraputic or } \\
\text { psychotropic } \\
\text { medications } \\
\text { - Additional mental } \\
\text { illnesses or organic } \\
\text { mental disorder } \\
\text { - Illicit drug and } \\
\text { alcohol abuse } \\
\text { - Clinically sig } \\
\text { medical disease } \\
\text { - Investigational drug } \\
\text { use } \\
\text { - ECT within last } 3 \\
\text { mos } \\
\text { - Suicidal tendencies } \\
\text { - Current episode } \\
\text { - } 12 \text { mos } \\
\text { - } 2 \text { previous } \\
\text { episodes of major } \\
\text { depression that did } \\
\text { not respond to AD } \\
\text { therapy } \\
\text { - }\end{array}$ & $\begin{array}{l}\text { Mean age (yrs): } \\
\text { D1: } 45.9 \\
\text { D2: } 44.5 \\
\text { Sex (\% female): } \\
\text { D1: } 62.8 \\
\text { D2: } 68.4 \\
\text { Race (\% white): } \\
\text { NR } \\
\text { Baseline (HAM-A): } \\
\text { NR } \\
\text { Mean HAM-D score } \\
\text { at baseline: } \\
\text { D1: } 29.5 \text { (3.0) } \\
\text { D2: } 29.2(2.9)\end{array}$ & $\begin{array}{l}\text { D1: Orgasm } \\
\text { dysfunction } 9 / 78 \\
\text { (11.8\%); Decreased } \\
\text { sexual desire: } 9 / 78 \\
(11.8 \%) ;(29 / 49, \\
\text { males/females);3 } \\
\text { ejaculatory overall SD: } \\
21 / 78(23.1 \%) \\
\text { D2: Orgasm } \\
\text { dysfunction } 9 / 79 \\
\text { (10.8\%); Decreased } \\
\text { sexual desire } 11 / 79 \\
\text { (13.5\%); (25/54 } \\
\text { males/females); } \\
\text { Ejaculatory } \\
\text { dysfunction (males) } \\
\text { 2/25 (9.1\% of men); } \\
\text { overall SD: } 20 / 79 \\
\text { (25.3\%) } \\
\text { Overall adverse } \\
\text { events: } \\
\text { D1: } 74.4 \\
\text { D2: } 65.8\end{array}$ & $\begin{array}{l}\text { UKU side effects } \\
\text { symptoms scale (UKU } \\
\text { symptoms with an } \\
\text { incidence of } 10 \% \text { in } \\
\text { either treatment group, } \\
\text { or a statistically } \\
\text { significant difference } \\
\text { between the groups) }\end{array}$ & $\begin{array}{l}\text { Overall attrition } \\
\text { rate: } \\
29.3 \% \\
\text { ITT analysis: } \\
\text { Yes } \\
\text { Study Quality: } \\
\text { Fair }\end{array}$ \\
\hline
\end{tabular}


Sexual Dysfunction associated with Second-Generation Antidepressants in Patients with Major Depressive Disorder - Results from a Systematic Review with Network Meta-Analysis. Drug Safety; U. Reichenpfader, G. Gartlehner, L.C. Morgan, A. Greenblatt, B. Nussbaumer, R. A. Hansen, M. Van Noord, L. Lux, and B. N. Gaynes. Corresponding author: U. Reichenpfader, Department for Evidence-based Medicine and Clinical Epidemiology, Krems, Austria; Department of Medical and Health Sciences - Division of Community Medicine, Linköping University, Sweden; ureichenpfader@hotmail.com

\begin{tabular}{|c|c|c|c|c|c|c|}
\hline $\begin{array}{c}\text { Study } \\
\text { Characteristics }\end{array}$ & $\begin{array}{l}\text { Research Objective } \\
\text { Study Design }\end{array}$ & Inclusion/Exclusion & $\begin{array}{c}\text { Baseline } \\
\text { Characteristics }\end{array}$ & $\begin{array}{l}\text { Sexual Dysfunction } \\
\text { (SD) Outcomes/ } \\
\text { Adverse Events }\end{array}$ & $\begin{array}{l}\text { Method used to } \\
\text { determine SD }\end{array}$ & $\begin{array}{c}\text { Analysis and Study } \\
\text { Quality (Risk of } \\
\text { Bias) }\end{array}$ \\
\hline $\begin{array}{l}\text { Author: } \\
\text { Hicks et al., } \\
2002[34] \\
\text { Country and } \\
\text { setting: } \\
\text { UK } \\
\text { Outpatient clinic } \\
\text { Funding: } \\
\text { Bristol Myers } \\
\text { Squibb }\end{array}$ & $\begin{array}{l}\text { Research objective: } \\
\text { Compare NEF and } \\
\text { PAR for treatment of } \\
\text { depression and sleep in } \\
\text { patients with mod- } \\
\text { severe MDD } \\
\text { Duration of study: } \\
8 \text { wks } \\
\text { Study design: } \\
\text { RCT } \\
\text { Overall study N: } \\
40 \\
\text { Intervention: } \\
\text { D1: NEF: } 400-600 \mathrm{mg} / \mathrm{d} \\
\text { D2: PAR: } 20-40 \mathrm{mg} / \mathrm{d}\end{array}$ & $\begin{array}{l}\text { Inclusion criteria: } \\
\text { - Adults } 18 \text { or older } \\
\text { - Diagnosed with } \\
\text { MDD according to } \\
\text { DSM-III or -IV } \\
\text { - Minimum HAM-D } \\
\text { score of } 18 \\
\text { Exclusion criteria: } \\
\text { - Pregnant } \\
\text { - Lactating } \\
\text { - Concomitant } \\
\text { psychotheraputic or } \\
\text { psychotropic } \\
\text { medications } \\
\text { - Additional mental } \\
\text { illnesses or organic } \\
\text { mental disorder } \\
\text { - Illicit drug and } \\
\text { alcohol abuse } \\
\text { - Investigational drug } \\
\text { use within last } 30 \\
\text { days } \\
\text { - Shift workers } \\
\text { - Current sleep } \\
\text { disorders }\end{array}$ & $\begin{array}{l}\text { Mean age (yrs): } \\
\text { D1: } 42.75 \\
\text { D2: } 42.95 \\
\text { Sex (\% female): } \\
\text { D1: } 60 \\
\text { D2: } 55 \\
\text { Race (\% white): } \\
\text { NR } \\
\text { Baseline (HAM-A): } \\
\text { NR } \\
\text { Mean HAM-D score } \\
\text { at baseline: } \\
\text { D1: } 22 \\
\text { D2: } 22.5\end{array}$ & $\begin{array}{l}\text { Sexual dysfunction } \\
\text { (\%): } \\
\text { D1:0 } \\
\text { D2: } 20\end{array}$ & $\begin{array}{l}\text { AE specifically } \\
\text { monitored from } \\
\text { checklist of symptoms }\end{array}$ & $\begin{array}{l}\text { Overall attrition } \\
\text { rate: } \\
20 \% \\
\text { ITT analysis: } \\
\text { Yes } \\
\text { Study Quality: } \\
\text { Fair }\end{array}$ \\
\hline
\end{tabular}


Sexual Dysfunction associated with Second-Generation Antidepressants in Patients with Major Depressive Disorder - Results from a Systematic Review with Network Meta-Analysis. Drug Safety; U. Reichenpfader, G. Gartlehner, L.C. Morgan, A. Greenblatt, B. Nussbaumer, R. A. Hansen, M. Van Noord, L. Lux, and B. N. Gaynes. Corresponding author: U. Reichenpfader, Department for Evidence-based Medicine and Clinical Epidemiology, Krems, Austria; Department of Medical and Health Sciences - Division of Community Medicine, Linköping University, Sweden; ureichenpfader@hotmail.com

\begin{tabular}{|c|c|c|c|c|c|c|}
\hline $\begin{array}{c}\text { Study } \\
\text { Characteristics }\end{array}$ & $\begin{array}{l}\text { Research Objective } \\
\text { Study Design }\end{array}$ & Inclusion/Exclusion & $\begin{array}{c}\text { Baseline } \\
\text { Characteristics }\end{array}$ & $\begin{array}{l}\text { Sexual Dysfunction } \\
\text { (SD) Outcomes/ } \\
\text { Adverse Events }\end{array}$ & $\begin{array}{l}\text { Method used to } \\
\text { determine SD }\end{array}$ & $\begin{array}{c}\text { Analysis and Study } \\
\text { Quality (Risk of } \\
\text { Bias) } \\
\end{array}$ \\
\hline $\begin{array}{l}\text { Author: } \\
\text { Hochstrasser et } \\
\text { al., 2001[35] } \\
\text { Country and } \\
\text { setting: } \\
\text { Multinational, } \\
\text { multicenter } \\
\text { Funding: } \\
\text { H. Lundbeck A/S }\end{array}$ & $\begin{array}{l}\text { Research objective: } \\
\text { To compare } \\
\text { prophylactic efficacy of } \\
\text { CIT vs. PBO in } \\
\text { unipolar, recurrent } \\
\text { depression following } \\
\text { response to treatment } \\
\text { with CIT in previous } \\
\text { study periods } \\
\text { Duration of study: } \\
\text { 48-77 wks } \\
\text { Study design: } \\
\text { RCT } \\
\text { Overall study N: } \\
\text { (For period III): } 269 \\
\text { Intervention: } \\
\text { D1: CIT: } 20,40 \text {, or } 60 \\
\text { mg (3 groups + } \\
\text { PBO) } \\
\text { D2: PBO }\end{array}$ & $\begin{array}{l}\text { Inclusion criteria: } \\
\text { - Adults } 18 \text { to } 65 \\
\text { - Diagnosed with } \\
\text { MDD according to } \\
\text { DSM-III or -IV } \\
\text { - MADRS } 222 \\
\text { - Two or more } \\
\text { previous depressive } \\
\text { episodes (one } \\
\text { within last } 5 \text { yrs) } \\
\text { Exclusion criteria: } \\
\text { - Pregnant } \\
\text { - Concomitant } \\
\text { psychotheraputic or } \\
\text { psychotropic } \\
\text { medications } \\
\text { - Additional mental } \\
\text { illnesses or organic } \\
\text { mental disorder } \\
\text { - Illicit drug and } \\
\text { alcohol abuse } \\
\text { - Clinically sig } \\
\text { medical disease } \\
\text { - ECT within last } 3 \\
\text { days to } 8 \text { wks } \\
\text { - Suicidial tendencies } \\
\text { - MADRS item } 10 \geq 5 \\
\text { - Current depressive } \\
\text { episode longer than } \\
6 \text { mos } \\
\text { - Family history of } \\
\text { bipolar disorder }\end{array}$ & $\begin{array}{l}\text { Mean age (yrs): } \\
\text { D1: } 43.8(9.7) \\
\text { D2: } 42.4(11.5) \\
\text { Sex (\% female): } \\
\text { D1: } 67.4 \\
\text { D2: } 75 \\
\text { Race (\% white): } \\
\text { NR } \\
\text { Baseline HAM-A: } \\
\text { NR } \\
\text { Baseline HAM-D: } \\
\text { NR }\end{array}$ & $\begin{array}{l}\text { D1: } S D<2.5 \% \\
\text { D2: } 0 \%\end{array}$ & open question & $\begin{array}{l}\text { Overall attrition } \\
\text { rate: } \\
\text { NR } \\
\text { ITT Analysis } \\
\text { Yes } \\
\text { Study Quality: } \\
\text { Fair }\end{array}$ \\
\hline
\end{tabular}


Sexual Dysfunction associated with Second-Generation Antidepressants in Patients with Major Depressive Disorder - Results from a Systematic Review with Network Meta-Analysis. Drug Safety; U. Reichenpfader, G. Gartlehner, L.C. Morgan, A. Greenblatt, B. Nussbaumer, R. A. Hansen, M. Van Noord, L. Lux, and B. N. Gaynes. Corresponding author: U. Reichenpfader, Department for Evidence-based Medicine and Clinical Epidemiology, Krems, Austria; Department of Medical and Health Sciences - Division of Community Medicine, Linköping University, Sweden; ureichenpfader@hotmail.com

\begin{tabular}{|c|c|c|c|c|c|c|}
\hline $\begin{array}{c}\text { Study } \\
\text { Characteristics }\end{array}$ & $\begin{array}{l}\text { Research Objective } \\
\text { Study Design }\end{array}$ & Inclusion/Exclusion & $\begin{array}{c}\text { Baseline } \\
\text { Characteristics }\end{array}$ & $\begin{array}{l}\text { Sexual Dysfunction } \\
\text { (SD) Outcomes/ } \\
\text { Adverse Events }\end{array}$ & $\begin{array}{l}\text { Method used to } \\
\text { determine SD }\end{array}$ & $\begin{array}{c}\text { Analysis and Study } \\
\text { Quality (Risk of } \\
\text { Bias) }\end{array}$ \\
\hline $\begin{array}{l}\text { Author: } \\
\text { Hypericum } \\
\text { Depression Trial } \\
\text { Study Group, } \\
2002 \text { [36] } \\
\text { Country and } \\
\text { setting: } \\
\text { USA, Multicenter } \\
\text { Funding: } \\
\text { Study grants; } \\
\text { study } \\
\text { medications } \\
\text { donated by } \\
\text { Lichtwer Pharma } \\
\text { and Pfizer; }\end{array}$ & $\begin{array}{l}\text { Research objective: } \\
\text { To test efficacy and } \\
\text { safety of a well- } \\
\text { characterized } \\
\text { Hypericum perforatum } \\
\text { extract (LI-160) against } \\
\text { placebo and sertraline } \\
\text { in patients with MDD } \\
\text { Duration of study: } \\
8 \text { wks } \\
\text { Study design: } \\
\text { RCT } \\
\text { Overall study N: } \\
340 \text { randomized } \\
\text { Intervention: } \\
\text { D1: PBO } \\
\text { D2: SER: } 50-100 \mathrm{mg} / \mathrm{d}\end{array}$ & $\begin{array}{l}\text { Inclusion criteria: } \\
\text { - Aged at least } 18 \\
\text { years } \\
\text { - Diagnosed with } \\
\text { MDD according to } \\
\text { DSM-IV } \\
\text { - Minimum HAM-D } \\
\text { total score of at } \\
\text { least } 20 \text { at } \\
\text { screening and } \\
\text { baseline visits } \\
\text { - Minimum Global } \\
\text { Assessment of } \\
\text { Functioning (GAF) } \\
\text { total score of at } \\
\text { least } 20 \\
\text { Exclusion criteria: } \\
\text { - score above } 2 \text { on } \\
\text { the HAM-D suicide } \\
\text { item, attempted } \\
\text { suicied in teh last } \\
\text { year; } \\
\text { - pregnancy or } \\
\text { planning pregnancy } \\
\text { or breastfeeding; } \\
\text { - liver disease or } \\
\text { unstable serious } \\
\text { disease } \\
\text { - daily use of } \\
\text { sertraline or } \\
\text { Hypericum in the } \\
\text { last } 4 \text { weeks prior to } \\
\text { study entry; } \\
\text { concomitant } \\
\text { psychotheraputic or } \\
\text { psychotropic } \\
\text { medications, dietary } \\
\text { supplements } \\
\text { - any current }\end{array}$ & $\begin{array}{l}\text { Groups similar at } \\
\text { baseline: } \\
\text { yes } \\
\text { Mean age (yrs): } \\
\text { D1: } 40.1 \\
\text { D2: } 43.9 \\
\text { Sex (\% female): } \\
\text { D1: } 66.4 \\
\text { D2: } 66.7 \\
\text { Race (\% white): } \\
\text { D1: } 75.9 \\
\text { D2: } 73.9 \\
\text { Baseline (HAM-D- } \\
\text { 17): } \\
\text { D1: } 22.7 \\
\text { D2: } 22.5 \\
\text { Mean GAF score at } \\
\text { baseline: } \\
\text { D1: } 54.1 \\
\text { D2: } 53.6\end{array}$ & $\begin{array}{l}\text { D1: } 13.8 \% \\
\text { D2: } 32 \%\end{array}$ & $\begin{array}{l}\text { spontaneously reported } \\
\text { or elicited (44-item } \\
\text { checklist) }\end{array}$ & $\begin{array}{l}\text { Overall attrition } \\
\text { rate: } \\
27.9 \% \\
\text { ITT analysis: } \\
\text { modified ITT } \\
\text { (excluding } \\
\text { participants not } \\
\text { fullfilling entry } \\
\text { criteria ( } \mathrm{n}=2 \text { ) } \\
\text { Study Quality: } \\
\text { fair }\end{array}$ \\
\hline
\end{tabular}


Sexual Dysfunction associated with Second-Generation Antidepressants in Patients with Major Depressive Disorder - Results from a Systematic Review with Network Meta-Analysis. Drug Safety; U. Reichenpfader, G. Gartlehner, L.C. Morgan, A. Greenblatt, B. Nussbaumer, R. A. Hansen, M. Van Noord, L. Lux, and B. N. Gaynes. Corresponding author: U. Reichenpfader, Department for Evidence-based Medicine and Clinical Epidemiology, Krems, Austria; Department of Medical and Health Sciences - Division of Community Medicine, Linköping University, Sweden; ureichenpfader@hotmail.com

\begin{tabular}{ll}
\hline & psychiatric disorder \\
& other than MDD as \\
& defined in DSM-IV, \\
- & current or past \\
& history of a manic or \\
& hypomanic episode, \\
& schizophrenia or \\
& any other psychotic \\
& disorder, mental \\
& retardation, organic \\
& mental disorders or \\
& mental disorders \\
& due to a general \\
& medical condition, \\
& any \\
- & substance abuse \\
& disorder within the \\
previous 6 months, & known \\
& hypersensitivity to \\
study medications \\
\hline
\end{tabular}


Sexual Dysfunction associated with Second-Generation Antidepressants in Patients with Major Depressive Disorder - Results from a Systematic Review with Network Meta-Analysis. Drug Safety; U. Reichenpfader, G. Gartlehner, L.C. Morgan, A. Greenblatt, B. Nussbaumer, R. A. Hansen, M. Van Noord, L. Lux, and B. N. Gaynes. Corresponding author: U. Reichenpfader, Department for Evidence-based Medicine and Clinical Epidemiology, Krems, Austria; Department of Medical and Health Sciences - Division of Community Medicine, Linköping University, Sweden; ureichenpfader@hotmail.com

\begin{tabular}{|c|c|c|c|c|c|c|}
\hline $\begin{array}{c}\text { Study } \\
\text { Characteristics }\end{array}$ & $\begin{array}{l}\text { Research Objective } \\
\text { Study Design }\end{array}$ & Inclusion/Exclusion & $\begin{array}{c}\text { Baseline } \\
\text { Characteristics }\end{array}$ & $\begin{array}{l}\text { Sexual Dysfunction } \\
\text { (SD) Outcomes/ } \\
\text { Adverse Events }\end{array}$ & $\begin{array}{l}\text { Method used to } \\
\text { determine SD }\end{array}$ & $\begin{array}{c}\text { Analysis and Study } \\
\text { Quality (Risk of } \\
\text { Bias) }\end{array}$ \\
\hline $\begin{array}{l}\text { Author: } \\
\text { Katona et al., } \\
2012 \text { [37] } \\
\text { Country and } \\
\text { setting: } \\
\text { Multicenter } \\
\text { (Canada, } \\
\text { Finland, } \\
\text { France, } \\
\text { Germany, } \\
\text { Sweden, Ukraine } \\
\text { and the USA), } \\
\text { psychiatric, } \\
\text { psychogeriatric } \\
\text { and } \\
\text { geriatric settings } \\
\text { Funding: } \\
\text { H. Lundbeck } \\
\text { A/S, Takeda } \\
\text { Pharmaceutical } \\
\text { Company }\end{array}$ & $\begin{array}{l}\text { Research objective: } \\
\text { To compare efficacy, } \\
\text { safety and tolerability of } \\
\text { Lu AA21004 at } 5 \\
\text { mg/day compared with } \\
\text { placebo over } 8 \text { weeks } \\
\text { in elderly patients with } \\
\text { MDD with duloxetine } \\
\text { (60mg/day) } \\
\text { as an active reference } \\
\text { Duration of study: } \\
8 \text { wks } \\
\text { Study design: } \\
\text { RCT, double-blind } \\
\text { Overall study N: } \\
\text { 296 } \\
\text { Intervention: } \\
\text { D1: PBO } \\
\text { D2: DUL: } 60 \mathrm{mg} / \mathrm{d}\end{array}$ & $\begin{array}{l}\text { Inclusion criteria: } \\
\text { - Aged at least } 65 \\
\text { years } \\
\text { - Diagnosed with } \\
\text { MDD according to } \\
\text { DSM-IV of at least } \\
\text { 4-weeks' duration } \\
\text { and with at least } \\
\text { one previous MDE } \\
\text { before the age of } 60 \\
\text { years } \\
\text { - Minimum MADRS } \\
\text { total score of at } \\
\text { least } 26 \text { at } \\
\text { screening and } \\
\text { baseline visits } \\
\text { Exclusion criteria: } \\
\text { - Concomitant } \\
\text { psychotheraputic or } \\
\text { psychotropic } \\
\text { medications } \\
\text { - Mini-Mental State } \\
\text { Examination } \\
\text { (MMSE) score of } \\
\text { less than } 24 \text { at } \\
\text { screening } \\
\text { - any current } \\
\text { psychiatric disorder } \\
\text { other than MDD as } \\
\text { defined in DSM-IV, } \\
\text { - current or past } \\
\text { history of a manic or } \\
\text { hypomanic episode, } \\
\text { schizophrenia or } \\
\text { any other psychotic } \\
\text { disorder, mental } \\
\text { retardation, organic } \\
\text { mental disorders or } \\
\text { mental disorders }\end{array}$ & $\begin{array}{l}\text { Groups similar at } \\
\text { baseline: } \\
\text { yes } \\
\text { Mean age (yrs): } \\
\text { D1: } 39 \\
\text { D2: } 40 \\
\text { Sex (\% female): } \\
\text { D1: } 70.3 \\
\text { D2: } 70.9 \\
\text { Race (\% white): } \\
\text { D1: } 95.9 \\
\text { D2: } 95.4 \\
\text { Baseline (HAMD-24): } \\
\text { D1: } 29.4 \\
\text { D2: } 28.5 \\
\text { Mean MADRS score } \\
\text { at baseline: } \\
\text { D1: } 30.3 \\
\text { D2: } 30.4\end{array}$ & $\begin{array}{l}\text { D1: } 0 \\
\text { D2: } 6 \text { patients (male) } \\
\text { 6/51 (11.8\% of men): } \\
\text { [abnormal orgasm and } \\
\text { erectile dysfunction } \\
\text { (1), decreased libido } \\
\text { and erectile } \\
\text { dysfunction (1), } \\
\text { erectile dysfunction } \\
\text { (1), delayed } \\
\text { ejaculation (3)] }\end{array}$ & $\begin{array}{l}\text { non-leading question to } \\
\text { ascertain AE; no } \\
\text { targeted questions/ no } \\
\text { specific instrument } \\
\text { used to assess SD }\end{array}$ & $\begin{array}{l}\text { Overall attrition } \\
\text { rate: } \\
\text { 13.2\% } \\
\text { ITT analysis: } \\
\text { modified intent-to- } \\
\text { treat set - the full- } \\
\text { analysis set (FAS), } \\
\text { comprising all } \\
\text { patients in the APTS } \\
\text { who had at least one } \\
\text { valid postbaseline } \\
\text { assessment of the } \\
\text { primary efficacy } \\
\text { variable (HAM-D24 } \\
\text { total score) } \\
\text { Risk of Bias: } \\
\text { low }\end{array}$ \\
\hline
\end{tabular}


Sexual Dysfunction associated with Second-Generation Antidepressants in Patients with Major Depressive Disorder - Results from a Systematic Review with Network Meta-Analysis. Drug Safety; U. Reichenpfader, G. Gartlehner, L.C. Morgan, A. Greenblatt, B. Nussbaumer, R. A. Hansen, M. Van Noord, L. Lux, and B. N. Gaynes. Corresponding author: U. Reichenpfader, Department for Evidence-based Medicine and Clinical Epidemiology, Krems, Austria; Department of Medical and Health Sciences - Division of Community Medicine, Linköping University, Sweden; ureichenpfader@hotmail.com

\begin{tabular}{|c|}
\hline $\begin{array}{l}\text { due to a general } \\
\text { medical condition, } \\
\text { any } \\
\text { - substance abuse } \\
\text { disorder within the } \\
\text { previous } 6 \text { months, } \\
\text { - presence or history } \\
\text { of a clinically } \\
\text { significant } \\
\text { neurological } \\
\text { - Suicidal tendencies } \\
\text { - elevated intraocular } \\
\text { pressure or at risk } \\
\text { for acute narrow } \\
\text { angle glaucoma, } \\
\text { known } \\
\text { hypersensitivity to } \\
\text { duloxetine, a } \\
\text { chronic liver } \\
\text { disease, a clinically } \\
\text { significant unstable }\end{array}$ \\
\hline
\end{tabular}


Sexual Dysfunction associated with Second-Generation Antidepressants in Patients with Major Depressive Disorder - Results from a Systematic Review with Network Meta-Analysis. Drug Safety; U. Reichenpfader, G. Gartlehner, L.C. Morgan, A. Greenblatt, B. Nussbaumer, R. A. Hansen, M. Van Noord, L. Lux, and B. N. Gaynes. Corresponding author: U. Reichenpfader, Department for Evidence-based Medicine and Clinical Epidemiology, Krems, Austria; Department of Medical and Health Sciences - Division of Community Medicine, Linköping University, Sweden; ureichenpfader@hotmail.com

\begin{tabular}{|c|c|c|c|c|c|c|}
\hline $\begin{array}{c}\text { Study } \\
\text { Characteristics }\end{array}$ & $\begin{array}{l}\text { Research Objective } \\
\text { Study Design }\end{array}$ & Inclusion/Exclusion & $\begin{array}{c}\text { Baseline } \\
\text { Characteristics }\end{array}$ & $\begin{array}{l}\text { Sexual Dysfunction } \\
\text { (SD) Outcomes/ } \\
\text { Adverse Events }\end{array}$ & $\begin{array}{l}\text { Method used to } \\
\text { determine SD }\end{array}$ & $\begin{array}{c}\text { Analysis and Study } \\
\text { Quality (Risk of } \\
\text { Bias) }\end{array}$ \\
\hline \multirow{4}{*}{$\begin{array}{l}\text { Author: } \\
\text { Keller et al., } \\
\text { 1998[38] } \\
\text { Country and } \\
\text { setting: } \\
\text { United States } \\
\text { (10) outpatient } \\
\text { psychiatric clinics } \\
\text { and (2) academic } \\
\text { centers }\end{array}$} & \multirow{4}{*}{$\begin{array}{l}\text { Research objective: } \\
\text { To determine if } \\
\text { maintenance therapy } \\
\text { with SER can } \\
\text { effectively prevent } \\
\text { recurrence of } \\
\text { depression in patients } \\
\text { with chronic major } \\
\text { depression or double } \\
\text { depression }\end{array}$} & \multirow{9}{*}{$\begin{array}{l}\text { Inclusion criteria: } \\
\text { - Diagnosed with } \\
\text { MDD according to } \\
\text { DSM-III or -IV } \\
\text { - Minimum HAM-D } \\
\text { score of } 18 \\
\text { - MDD with or without } \\
\text { dysthymic disorder } \\
\text { - Chronic depression } \\
\text { defined as } \\
\text { depression of at } \\
\text { least } 2 \text { yrs duration } \\
\text { - } 3 \text { phase study } \\
\text { Exclusion criteria: } \\
\text { NR }\end{array}$} & $\begin{array}{l}\text { Mean age (yrs): } \\
\text { D1: } 40.8 \\
\text { D2: } 42.4\end{array}$ & \multirow[t]{9}{*}{$\begin{array}{l}\text { Sexual dysfunctional } \\
\text { (male ejaculation): } \\
\text { D1: } 13 / 77(16.9 \%) \\
\text { D2: } 0\end{array}$} & NR & $\begin{array}{l}\text { Overall attrition } \\
\text { rate: } \\
63.4 \%\end{array}$ \\
\hline & & & $\begin{array}{l}\text { Sex (\% female): } \\
\text { D1: } 62 \\
\text { D2: } 69\end{array}$ & & & $\begin{array}{l}\text { ITT Analysis } \\
\text { No, time to event of } \\
\text { full population }\end{array}$ \\
\hline & & & $\begin{array}{l}\text { Race (\% white): } \\
\text { NR }\end{array}$ & & & $\begin{array}{l}\text { Study Quality: } \\
\text { Fair }\end{array}$ \\
\hline & & & Baseline HAM-A: & & & \\
\hline \multirow{5}{*}{$\begin{array}{l}\text { Funding: } \\
\text { Pfizer }\end{array}$} & Duration of study: & & NR & & & \\
\hline & & & Baseline HAM-D: & & & \\
\hline & RCT & & $\begin{array}{l}\text { D1: } 5.5(4.2) \\
\text { D2: } 6.3(3.7)\end{array}$ & & & \\
\hline & $\begin{array}{l}\text { Overall study } \mathbf{N}: \\
161\end{array}$ & & & & & \\
\hline & $\begin{array}{l}\text { Intervention: } \\
\text { D1: SER: } 50-200 \mathrm{mg} / \mathrm{d} \\
\text { D2: PBO }\end{array}$ & & & & & \\
\hline
\end{tabular}


Sexual Dysfunction associated with Second-Generation Antidepressants in Patients with Major Depressive Disorder - Results from a Systematic Review with Network Meta-Analysis. Drug Safety; U. Reichenpfader, G. Gartlehner, L.C. Morgan, A. Greenblatt, B. Nussbaumer, R. A. Hansen, M. Van Noord, L. Lux, and B. N. Gaynes. Corresponding author: U. Reichenpfader, Department for Evidence-based Medicine and Clinical Epidemiology, Krems, Austria; Department of Medical and Health Sciences - Division of Community Medicine, Linköping University, Sweden; ureichenpfader@hotmail.com

\begin{tabular}{|c|c|c|c|c|c|c|}
\hline $\begin{array}{c}\text { Study } \\
\text { Characteristics }\end{array}$ & $\begin{array}{l}\text { Research Objective } \\
\text { Study Design }\end{array}$ & Inclusion/Exclusion & $\begin{array}{c}\text { Baseline } \\
\text { Characteristics }\end{array}$ & $\begin{array}{l}\text { Sexual Dysfunction } \\
\text { (SD) Outcomes/ } \\
\text { Adverse Events } \\
\end{array}$ & $\begin{array}{l}\text { Method used to } \\
\text { determine SD }\end{array}$ & $\begin{array}{c}\text { Analysis and Study } \\
\text { Quality (Risk of } \\
\text { Bias) }\end{array}$ \\
\hline $\begin{array}{l}\text { Author: } \\
\text { Kiev and Feiger, } \\
\text { 1997[39] } \\
\text { Country and } \\
\text { setting: } \\
\text { United States } \\
\text { Multicenter } \\
\text { (2 centers) } \\
\text { Funding: } \\
\text { Solvay } \\
\text { Pharmaceuticals, } \\
\text { Upjohn }\end{array}$ & $\begin{array}{l}\text { Research objective: } \\
\text { To compare FLUV and } \\
\text { PAR in treatment of } \\
\text { outpatients with major } \\
\text { depression } \\
\text { Duration of study: } \\
7 \text { wks } \\
\text { Study design: } \\
\text { RCT } \\
\text { Overall study } \mathrm{N}: \\
60 \\
\text { Intervention: } \\
\text { D1: FLUV: } 50-150 \mathrm{mg} / \mathrm{d} \\
\text { D2: PAR: } 20-50 \mathrm{mg} / \mathrm{d}\end{array}$ & $\begin{array}{l}\text { Inclusion criteria: } \\
\text { - Adults } 18 \text { to } 65 \\
\text { - MDD diagnosis } \\
\text { according to DSM- } \\
\text { III or -IV } \\
\text { - Minimum HAM-D } \\
\text { score of 20; } \\
\text { minimum score of } 2 \\
\text { on "depressed } \\
\text { mood" item } \\
\text { Exclusion criteria: } \\
\text { - Pregnant } \\
\text { - Lactating } \\
\text { - Concomitant } \\
\text { psychotheraputic or } \\
\text { psychotropic } \\
\text { medications } \\
\text { - Additional mental } \\
\text { illnesses or organic } \\
\text { mental disorder } \\
\text { - Illicit drug and } \\
\text { alcohol abuse } \\
\text { - Clinically sig } \\
\text { medical disease } \\
\text { - Suicidal tendencies } \\
\text { - Used a drug within } \\
\text { 30 days with } \\
\text { anticipated major } \\
\text { organ toxicity } \\
\text { - Participation in } \\
\text { previous FLUV } \\
\text { studies } \\
\text { - Transportation } \\
\text { difficulties } \\
\text { - }\end{array}$ & $\begin{array}{l}\text { Mean age (yrs): } \\
\text { D1: } 42.7 \\
\text { D2: } 39.9 \\
\text { Sex (\% female): } \\
\text { D1: } 53 \\
\text { D2: } 53 \\
\text { Race (\% white): } \\
\text { D1: } 87 \\
\text { D2: } 93 \\
\text { Baseline (HAM-A): } \\
\text { NR } \\
\text { Mean HAM-D score } \\
\text { at baseline: } \\
\text { D1: } 24.35 \\
\text { D2: } 24.36\end{array}$ & $\begin{array}{l}\text { Sexual dysfunction: } \\
\text { D1: ejaculation } \\
\text { abnormality: } 1 / 14 \\
\text { (7.1\% of men); } \\
\text { decreased libido: } \\
\text { 4/30; total SD } 5 / 30 \\
\text { (16.7\%) } \\
\text { D2: ejaculation } \\
\text { abnormality: } 3 / 14 \\
\text { (21.4\% of men); } \\
\text { decreased libido: } 5 / 30 \\
\text { (16.7\%); total SD: } \\
8 / 30 \text { (26.7\%) } \\
\text { Overall adverse } \\
\text { events: } \\
\text { D1: } 97 \\
\text { D2: } 100\end{array}$ & $\begin{array}{l}\text { observed or } \\
\text { spontaneously/ reported } \\
\mathrm{AE}\end{array}$ & $\begin{array}{l}\text { Overall attrition } \\
\text { rate: } \\
31 \% \\
\text { ITT analysis: } \\
\text { Yes } \\
\text { Study Quality: } \\
\text { Fair }\end{array}$ \\
\hline
\end{tabular}


Sexual Dysfunction associated with Second-Generation Antidepressants in Patients with Major Depressive Disorder - Results from a Systematic Review with Network Meta-Analysis. Drug Safety; U. Reichenpfader, G. Gartlehner, L.C. Morgan, A. Greenblatt, B. Nussbaumer, R. A. Hansen, M. Van Noord, L. Lux, and B. N. Gaynes. Corresponding author: U. Reichenpfader, Department for Evidence-based Medicine and Clinical Epidemiology, Krems, Austria; Department of Medical and Health Sciences - Division of Community Medicine, Linköping University, Sweden; ureichenpfader@hotmail.com

\begin{tabular}{|c|c|c|c|c|c|c|}
\hline $\begin{array}{c}\text { Study } \\
\text { Characteristics }\end{array}$ & $\begin{array}{l}\text { Research Objective } \\
\text { Study Design }\end{array}$ & Inclusion/Exclusion & $\begin{array}{c}\text { Baseline } \\
\text { Characteristics }\end{array}$ & $\begin{array}{l}\text { Sexual Dysfunction } \\
\text { (SD) Outcomes/ } \\
\text { Adverse Events } \\
\end{array}$ & $\begin{array}{l}\text { Method used to } \\
\text { determine SD }\end{array}$ & $\begin{array}{c}\text { Analysis and Study } \\
\text { Quality (Risk of } \\
\text { Bias) }\end{array}$ \\
\hline $\begin{array}{l}\text { Author: } \\
\text { Lepola et al., } \\
\text { 2003[40] }\end{array}$ & $\begin{array}{l}\text { Research objective: } \\
\text { Efficacy and tolerability } \\
\text { of ESC compared to } \\
\text { CIT and PBO in }\end{array}$ & $\begin{array}{l}\text { Inclusion criteria: } \\
\text { - Adults } 18 \text { to } 65 \\
\text { - Diagnosed with } \\
\text { MDD according to }\end{array}$ & $\begin{array}{l}\text { Mean age (yrs): } \\
\text { D1: } 43 \\
\text { D2: } 43 \\
\text { D3: } 43\end{array}$ & $\begin{array}{l}\text { Sexual dysfunction: } \\
\text { D1: } 0 \\
\text { D2: } 2 / 39(5.6 \%) \\
\text { D3: } 0\end{array}$ & \multirow[t]{7}{*}{ NR } & $\begin{array}{l}\text { Overall attrition } \\
\text { rate: } \\
7 \%\end{array}$ \\
\hline $\begin{array}{l}\text { Country and } \\
\text { setting: }\end{array}$ & $\begin{array}{l}\text { depression in primary } \\
\text { care setting }\end{array}$ & DSM-III or -IV & Sex (\% female): & \multirow{6}{*}{$\begin{array}{l}\text { Overall advers } \\
\text { events: } \\
\text { D1: } 59.7 \\
\text { D2: } 69.7 \\
\text { D3: } 65\end{array}$} & & $\begin{array}{l}\text { ITT analysis: } \\
\text { Yes }\end{array}$ \\
\hline $\begin{array}{l}\text { Europe and } \\
\text { Canada }\end{array}$ & Duration of study: & Evalucion = & $\begin{array}{l}\text { D1: } 69.4 \\
\text { D2: } 74.8\end{array}$ & & & Study Quality: \\
\hline Primary care & 8 wks & - Pregnant & D3: 72.1 & & & Fair \\
\hline \multirow[t]{3}{*}{$\begin{array}{l}\text { Funding: } \\
\text { H. Lundbeck A/S }\end{array}$} & $\begin{array}{l}\text { Study design: } \\
\text { RCT }\end{array}$ & - Lactating & $\begin{array}{l}\text { Race (\% white): } \\
\text { NR }\end{array}$ & & & \\
\hline & $\begin{array}{l}\text { Overall study } \mathbf{N}: \\
315\end{array}$ & $\begin{array}{l}\text { psychotropic } \\
\text { medications }\end{array}$ & $\begin{array}{l}\text { Baseline (HAM-A): } \\
\text { NR }\end{array}$ & & & \\
\hline & $\begin{array}{l}\text { Intervention: } \\
\text { D1: CIT: } 20-40 \mathrm{mg} / \mathrm{d} \\
\quad \text { (mean } 28.4) \\
\text { D2: ESC: } 10-20 \mathrm{mg} / \mathrm{d} \\
\quad \text { (mean 14.0) } \\
\text { D3: PBO }\end{array}$ & $\begin{array}{l}\text { - Additional mental } \\
\text { illnesses or organic } \\
\text { mental disorder } \\
\text { - Illicit drug and } \\
\text { alcohol abuse } \\
\text { - Suicidal tendencies }\end{array}$ & $\begin{array}{l}\text { Mean HAM-D score } \\
\text { at baseline: } \\
\text { NR }\end{array}$ & & & \\
\hline
\end{tabular}


Sexual Dysfunction associated with Second-Generation Antidepressants in Patients with Major Depressive Disorder - Results from a Systematic Review with Network Meta-Analysis. Drug Safety; U. Reichenpfader, G. Gartlehner, L.C. Morgan, A. Greenblatt, B. Nussbaumer, R. A. Hansen, M. Van Noord, L. Lux, and B. N. Gaynes. Corresponding author: U. Reichenpfader, Department for Evidence-based Medicine and Clinical Epidemiology, Krems, Austria; Department of Medical and Health Sciences - Division of Community Medicine, Linköping University, Sweden; ureichenpfader@hotmail.com

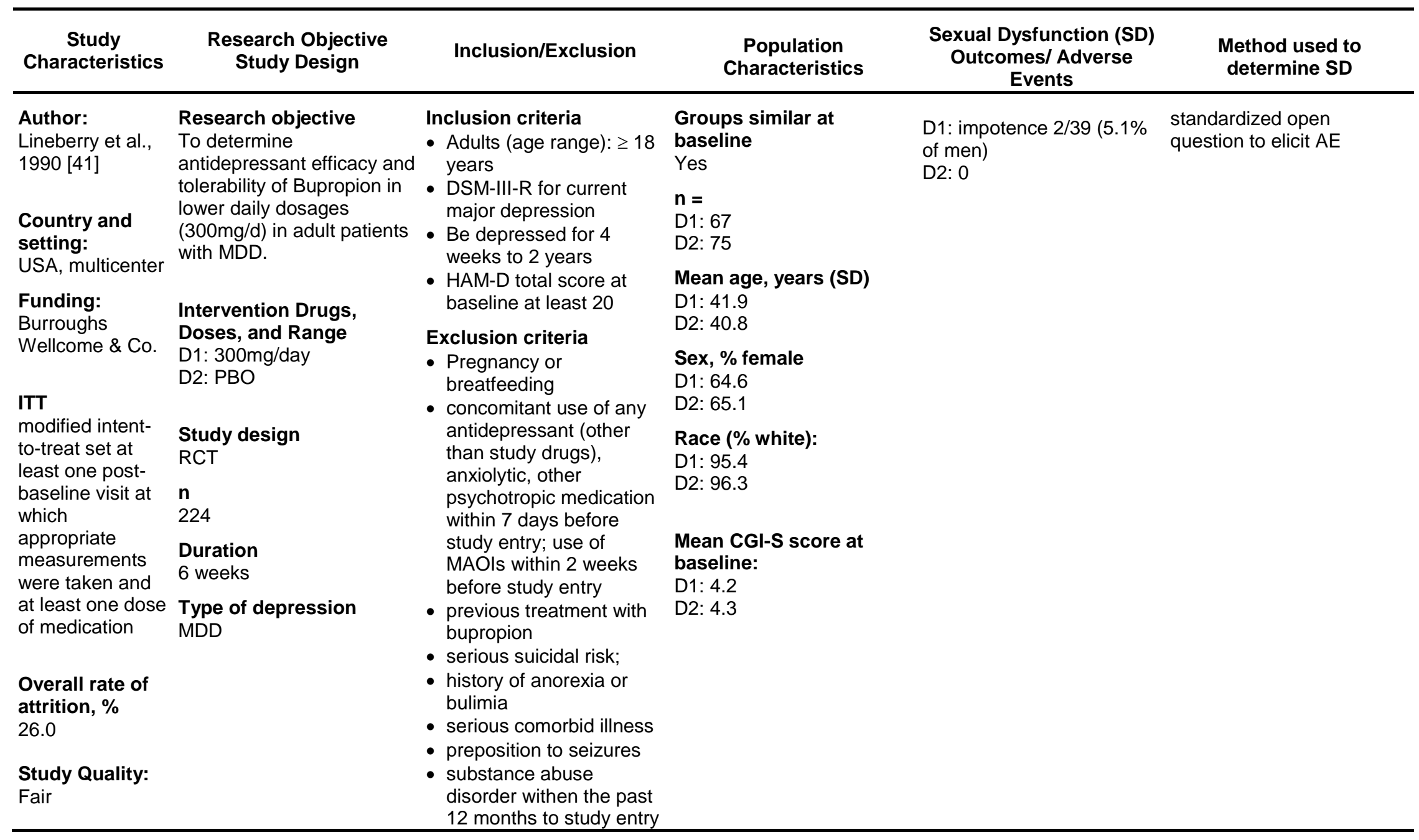


Sexual Dysfunction associated with Second-Generation Antidepressants in Patients with Major Depressive Disorder - Results from a Systematic Review with Network Meta-Analysis. Drug Safety; U. Reichenpfader, G. Gartlehner, L.C. Morgan, A. Greenblatt, B. Nussbaumer, R. A. Hansen, M. Van Noord, L. Lux, and B. N. Gaynes. Corresponding author: U. Reichenpfader, Department for Evidence-based Medicine and Clinical Epidemiology, Krems, Austria; Department of Medical and Health Sciences - Division of Community Medicine, Linköping University, Sweden; ureichenpfader@hotmail.com

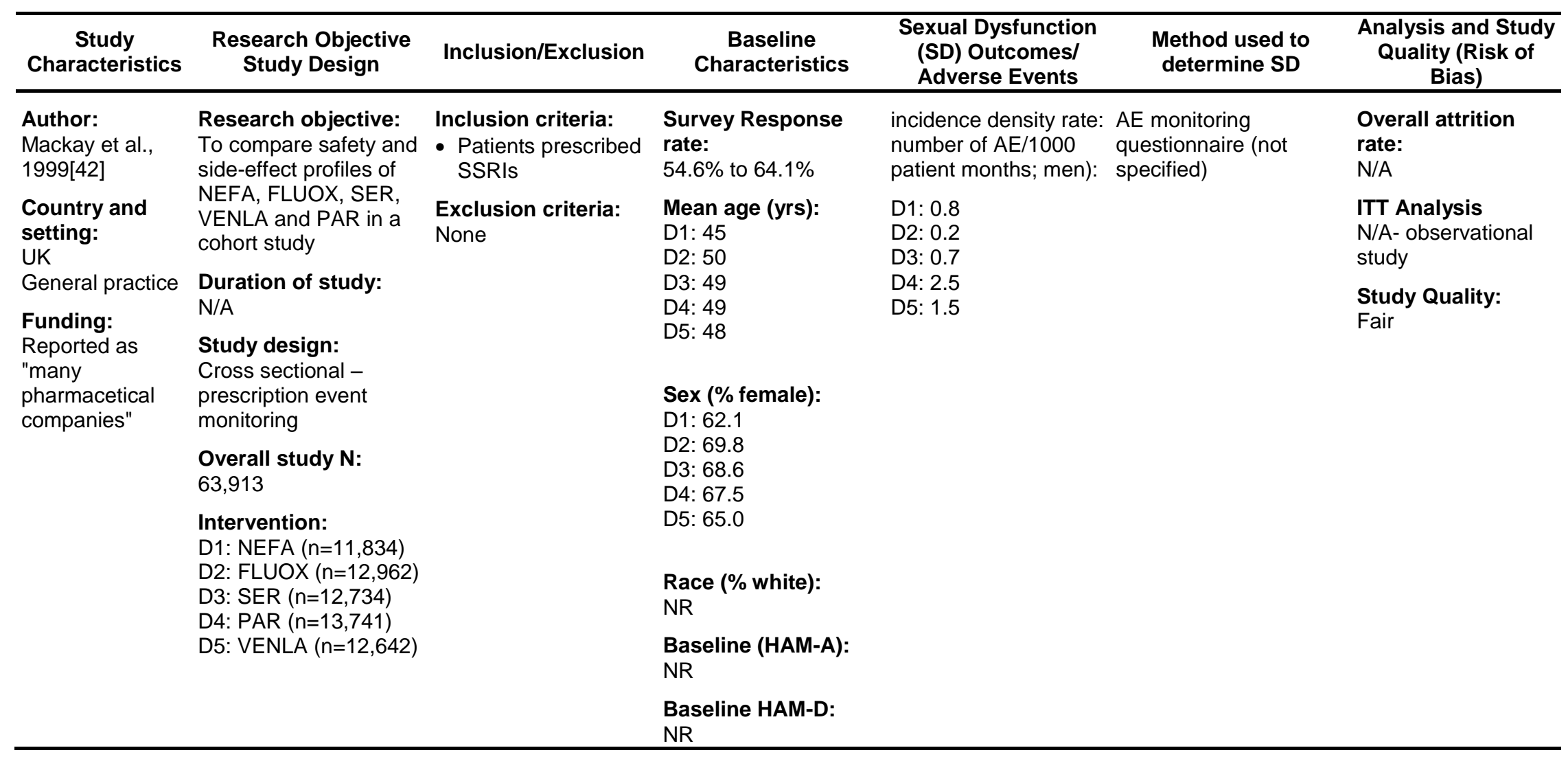


Sexual Dysfunction associated with Second-Generation Antidepressants in Patients with Major Depressive Disorder - Results from a Systematic Review with Network Meta-Analysis. Drug Safety; U. Reichenpfader, G. Gartlehner, L.C. Morgan, A. Greenblatt, B. Nussbaumer, R. A. Hansen, M. Van Noord, L. Lux, and B. N. Gaynes. Corresponding author: U. Reichenpfader, Department for Evidence-based Medicine and Clinical Epidemiology, Krems, Austria; Department of Medical and Health Sciences - Division of Community Medicine, Linköping University, Sweden; ureichenpfader@hotmail.com

\begin{tabular}{|c|c|c|c|c|c|c|}
\hline $\begin{array}{c}\text { Study } \\
\text { Characteristics }\end{array}$ & $\begin{array}{l}\text { Research Objective } \\
\text { Study Design }\end{array}$ & Inclusion/Exclusion & $\begin{array}{c}\text { Baseline } \\
\text { Characteristics }\end{array}$ & $\begin{array}{c}\text { Sexual Dysfunction } \\
\text { (SD) Outcomes/ } \\
\text { Adverse Events } \\
\end{array}$ & $\begin{array}{l}\text { Method used to } \\
\text { determine SD }\end{array}$ & $\begin{array}{c}\text { Analysis and Study } \\
\text { Quality (Risk of } \\
\text { Bias) }\end{array}$ \\
\hline $\begin{array}{l}\text { Author: } \\
\text { Mehtonen et al., } \\
\text { 2000[43] } \\
\text { Country and } \\
\text { setting: } \\
\text { Scandinavia } \\
\text { Multicenter } \\
\text { Funding: } \\
\text { Wyeth-Ayerst } \\
\text { International }\end{array}$ & $\begin{array}{l}\text { Research objective: } \\
\text { Efficacy and safety of } \\
\text { SER and VEN in } \\
\text { outpatients with major } \\
\text { depression } \\
\text { Duration of study: } \\
8 \text { wks } \\
\text { Study design: } \\
\text { RCT } \\
\text { Overall study N: } \\
147 \\
\text { Intervention: } \\
\text { D1: VEN: } 75-150 \mathrm{mg} / \mathrm{d} \\
\text { D2: SER: } 50-100 \mathrm{mg} / \mathrm{d}\end{array}$ & $\begin{array}{l}\text { Inclusion criteria: } \\
\text { - Adults } 18 \text { to } 65 \\
\text { - Minimum HAM-D } \\
\text { score of } 18 \\
\text { Exclusion criteria: } \\
\text { - Pregnant } \\
\text { - Lactating } \\
\text { - Additional mental } \\
\text { illnesses or organic } \\
\text { mental disorder } \\
\text { - Illicit drug and } \\
\text { alcohol abuse } \\
\text { - Clinically sig } \\
\text { medical disease }\end{array}$ & $\begin{array}{l}\text { Mean age (yrs): } \\
\text { D1: } 44.1 \\
\text { D2: } 41.0 \\
\text { Sex (\% female): } \\
\text { D1: } 65 \\
\text { D2: } 67 \\
\text { Race (\% white): } \\
\text { NR } \\
\text { Baseline (HAM-A): } \\
\text { NR } \\
\text { Mean HAM-D score } \\
\text { at baseline: } \\
\text { D1: } 25.5(3.5) \\
\text { D2: } 25.8 \text { (4.5) }\end{array}$ & $\begin{array}{l}\text { Sexual dysfunction } \\
\text { (including } \\
\text { anorgasmia, } \\
\text { impotence/erectile } \\
\text { dysfunction, } \\
\text { abnormal } \\
\text { orgasm/ejaculation, } \\
\text { increased/decreased } \\
\text { libido): } \\
\text { D1: } 6 / 75(8 \%) \\
\text { D2: } 4 / 72(5.6 \%)\end{array}$ & $\begin{array}{l}\text { UKU side effect rating } \\
\text { scale }\end{array}$ & $\begin{array}{l}\text { Overall attrition } \\
\text { rate: } \\
19 \% \\
\text { ITT analysis: } \\
\text { Yes } \\
\text { Study Quality: } \\
\text { Good }\end{array}$ \\
\hline
\end{tabular}


Sexual Dysfunction associated with Second-Generation Antidepressants in Patients with Major Depressive Disorder - Results from a Systematic Review with Network Meta-Analysis. Drug Safety; U. Reichenpfader, G. Gartlehner, L.C. Morgan, A. Greenblatt, B. Nussbaumer, R. A. Hansen, M. Van Noord, L. Lux, and B. N. Gaynes. Corresponding author: U. Reichenpfader, Department for Evidence-based Medicine and Clinical Epidemiology, Krems, Austria; Department of Medical and Health Sciences - Division of Community Medicine, Linköping University, Sweden; ureichenpfader@hotmail.com

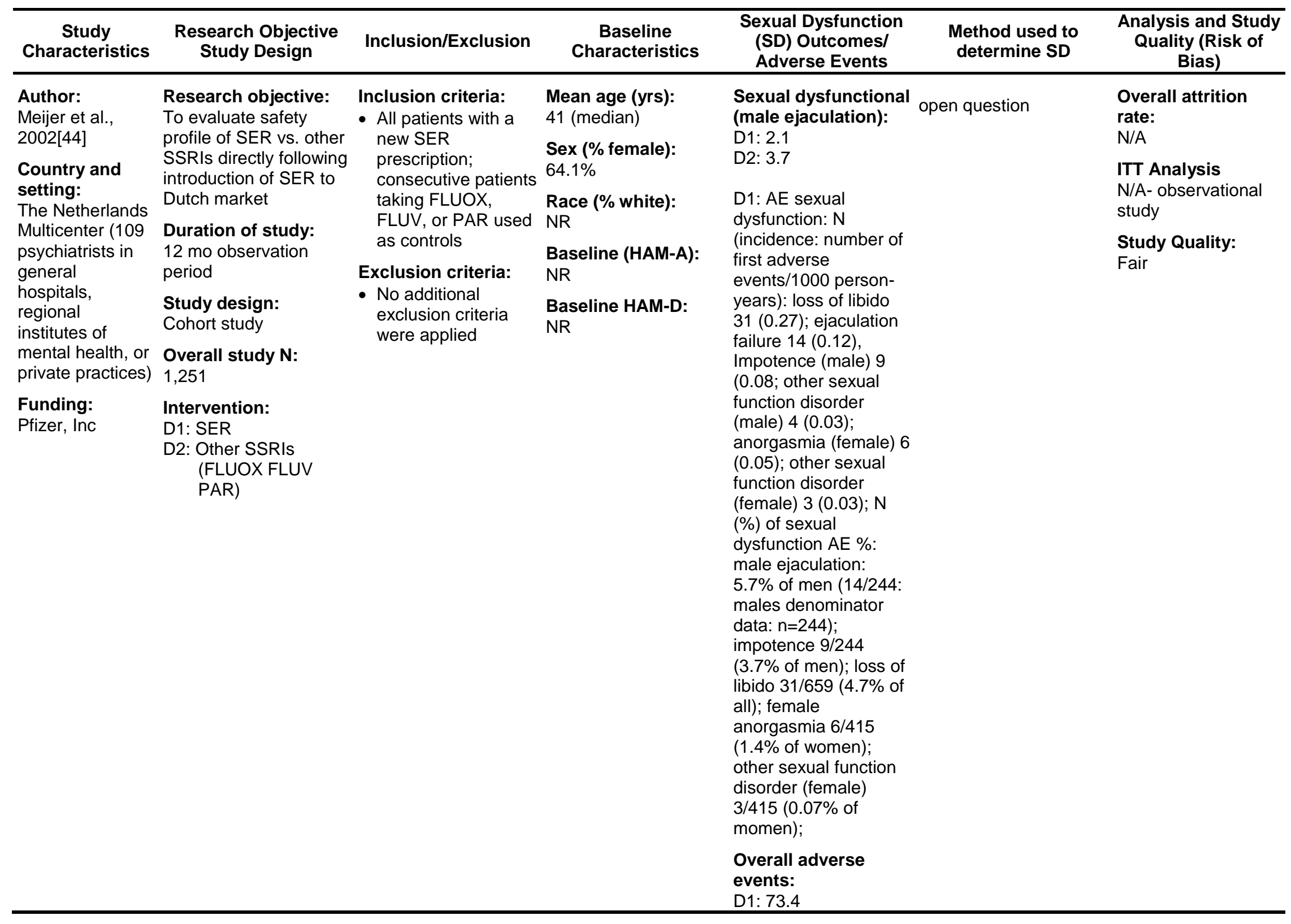


Sexual Dysfunction associated with Second-Generation Antidepressants in Patients with Major Depressive Disorder - Results from a Systematic Review with Network Meta-Analysis. Drug Safety; U. Reichenpfader, G. Gartlehner, L.C. Morgan, A. Greenblatt, B. Nussbaumer, R. A. Hansen, M. Van Noord, L. Lux, and B. N. Gaynes. Corresponding author: U. Reichenpfader, Department for Evidence-based Medicine and Clinical Epidemiology, Krems, Austria; Department of Medical and Health Sciences - Division of Community Medicine, Linköping University, Sweden; ureichenpfader@hotmail.com

\begin{tabular}{|c|c|c|c|c|c|c|}
\hline $\begin{array}{c}\text { Study } \\
\text { Characteristics }\end{array}$ & $\begin{array}{c}\text { Research Objective } \\
\text { Study Des }\end{array}$ & Inclusion/Exclusion & $\begin{array}{c}\text { Baseline } \\
\text { Characteristics }\end{array}$ & $\begin{array}{l}\text { Sexual Dysfunction } \\
\text { (SD) Outcomes/ } \\
\text { Adverse Events }\end{array}$ & $\begin{array}{l}\text { Method used to } \\
\text { determine SD }\end{array}$ & $\begin{array}{c}\text { Analysis and Study } \\
\text { Quality (Risk of } \\
\text { Bias) }\end{array}$ \\
\hline $\begin{array}{l}\text { Author: } \\
\text { Montejo et al., } \\
\text { 2001[45] } \\
\text { Country and } \\
\text { setting: } \\
\text { Spain } \\
\text { Multicenter } \\
\text { Funding: } \\
\text { Bristol-Myers } \\
\text { Squibb }\end{array}$ & $\begin{array}{l}\text { Research objective: } \\
\text { Incidence of sexual } \\
\text { dysfunction associated } \\
\text { with anti-depressant } \\
\text { agents } \\
\text { Duration of study: } \\
\text { Carried out between } \\
\text { April } 1995 \text { and } \\
\text { February } 2000 \\
\text { Study design: } \\
\text { Prospective cohort } \\
\text { study } \\
\text { Overall study N: } \\
1,022 \\
\text { Intervention: } \\
\text { CIT } \\
\text { FLUOX } \\
\text { FLUV } \\
\text { MIR } \\
\text { NEF } \\
\text { PAR } \\
\text { SER } \\
\text { VEN }\end{array}$ & $\begin{array}{l}\text { Inclusion criteria: } \\
\text { - Normal sexual } \\
\text { functioning prior to } \\
\text { taking } \\
\text { antidepressants } \\
\text { - Treatment with } \\
\text { antidepressant } \\
\text { alone or combine } \\
\text { with benzodiazepine } \\
\text { - Previous regular } \\
\text { and satisfactory } \\
\text { sexual practices } \\
\text { - Occurrence of } \\
\text { sexual dysfunction } \\
\text { within } 2 \text { mos after } \\
\text { introduction of } \\
\text { antidepressant } \\
\text { Exclusion criteria: } \\
\text { - Prior sexual } \\
\text { dysfunction } \\
\text { - Combination of } \\
\text { antidepressant and } \\
\text { neuroleptic } \\
\text { treatment } \\
\text { - Treatment with } \\
\text { hormones or any } \\
\text { other drug capable } \\
\text { of interfering with } \\
\text { sexual intercourse } \\
\text { - Sig intercurrent } \\
\text { diseases affecting } \\
\text { sexual function } \\
\text { - Substance abuse }\end{array}$ & $\begin{array}{l}\text { Mean age (yrs): } \\
\text { Overall: } 39.8 \\
\text { Sex (\% female): } \\
\text { Overall: } 60 \\
\text { Race (\% white): } \\
\text { NR } \\
\text { Baseline (HAM-A): } \\
\text { NR } \\
\text { Baseline HAM-D: } \\
\text { NR } \\
\end{array}$ & $\begin{array}{l}\text { Overall incidence of } \\
\text { sexual dysfunction } \\
\text { was } 59.1 \% \\
\text { Incidence of overall } \\
\text { sexual dysfunction: } \\
\text { FLUOX, } 57.7 \% \\
\text { SER, } 62.9 \% \\
\text { FLUV, } 62.3 \% \\
\text { PAR, } 70.7 \% \\
\text { CIT, } 72.7 \% \\
\text { VEN, } 67.3 \% \\
\text { MIR, } 24.4 \% \\
\text { NEF, } 8 \% \\
\text { Men had a higher } \\
\text { frequency of sexual } \\
\text { dysfunction (62.4\%) } \\
\text { than women (56.9\%), } \\
\text { although women had } \\
\text { higher severity }\end{array}$ & $\begin{array}{l}\text { Psychotropic-Related } \\
\text { Sexual Dysfunction } \\
\text { Questionnaire } \\
\text { (PRSexDQ) }\end{array}$ & $\begin{array}{l}\text { Overall attrition } \\
\text { rate: } \\
\text { N/A } \\
\text { ITT Analysis } \\
\text { Not applicable- } \\
\text { observational study } \\
\text { Study Quality: } \\
\text { Fair }\end{array}$ \\
\hline
\end{tabular}


Sexual Dysfunction associated with Second-Generation Antidepressants in Patients with Major Depressive Disorder - Results from a Systematic Review with Network Meta-Analysis. Drug Safety; U. Reichenpfader, G. Gartlehner, L.C. Morgan, A. Greenblatt, B. Nussbaumer, R. A. Hansen, M. Van Noord, L. Lux, and B. N. Gaynes. Corresponding author: U. Reichenpfader, Department for Evidence-based Medicine and Clinical Epidemiology, Krems, Austria; Department of Medical and Health Sciences - Division of Community Medicine, Linköping University, Sweden; ureichenpfader@hotmail.com

\begin{tabular}{|c|c|c|c|c|c|c|}
\hline $\begin{array}{c}\text { Study } \\
\text { Characteristics }\end{array}$ & $\begin{array}{l}\text { Research Objective } \\
\text { Study Design }\end{array}$ & Inclusion/Exclusion & $\begin{array}{c}\text { Baseline } \\
\text { Characteristics }\end{array}$ & $\begin{array}{c}\text { Sexual Dysfunction } \\
\text { (SD) Outcomes/ } \\
\text { Adverse Events } \\
\end{array}$ & $\begin{array}{l}\text { Method used to } \\
\text { determine SD }\end{array}$ & $\begin{array}{c}\text { Analysis and Study } \\
\text { Quality (Risk of } \\
\text { Bias) }\end{array}$ \\
\hline $\begin{array}{l}\text { Author: } \\
\text { Montgomery et } \\
\text { al., 2004[46] } \\
\text { Country and } \\
\text { setting: } \\
\text { Mulitnational } \\
\text { Primary care } \\
\text { Funding: } \\
\text { H. Lundbeck A/S }\end{array}$ & $\begin{array}{l}\text { Research objective: } \\
\text { To compare efficacy } \\
\text { and tolerability of ESC } \\
\text { to VEN XR in primary } \\
\text { care patients with MDD } \\
\text { Duration of study: } \\
8 \text { wks } \\
\text { Study design: } \\
\text { RCT } \\
\text { Overall study N: } \\
\text { 297 } \\
\text { Intervention: } \\
\text { D1: ESC: } 10-20 \mathrm{mg} / \mathrm{d} \\
\text { (12.1) } \\
\text { D2: VEN: } 75-150 \mathrm{mg} / \mathrm{d} \\
\text { (95.2) }\end{array}$ & $\begin{array}{l}\text { Inclusion criteria: } \\
\text { - Adults } 18 \text { to } 85 \\
\text { - Diagnosed with } \\
\text { MDD according to } \\
\text { DSM-III or -IV } \\
\text { - MADRS } \geq 18 \\
\text { Exclusion criteria: } \\
\text { - Pregnant } \\
\text { - Lactating } \\
\text { - Concomitant } \\
\text { psychotheraputic or } \\
\text { psychotropic } \\
\text { medications } \\
\text { - Additional mental } \\
\text { illnesses or organic } \\
\text { mental disorder } \\
\text { - Illicit drug and } \\
\text { alcohol abuse } \\
\text { - Clinically sig } \\
\text { medical disease } \\
\text { - Suicidal tendencies }\end{array}$ & $\begin{array}{l}\text { Mean age (yrs): } \\
\text { D1: } 49 \\
\text { D2: } 47 \\
\text { Sex (\% female): } \\
\text { D1: } 73 \\
\text { D2: } 71 \\
\text { Race (\% white): } \\
\text { NR } \\
\text { Baseline (HAM-A): } \\
\text { NR } \\
\text { Mean HAM-D score } \\
\text { at baseline: } \\
\text { D1: } 19.9 \\
\text { D2: } 20.4\end{array}$ & $\begin{array}{l}\text { male } \\
\text { impotence/erectile } \\
\text { dysfunction (data only } \\
\text { shown in bar chart, } \\
\text { reported in } \\
\text { Montgomery \& } \\
\text { Andersen, 2006) } \\
\text { D1: } 5.1 \% \\
\text { D2: } 2.4 \%\end{array}$ & $\begin{array}{l}\text { AE spontaneously } \\
\text { reported; } \\
\text { Discontinuation } \\
\text { Emergent Signs and } \\
\text { Symptoms Checklist } \\
\text { (DESS) checklist }\end{array}$ & $\begin{array}{l}\text { Overall attrition } \\
\text { rate: } \\
14 \% \\
\text { ITT analysis: } \\
\text { Yes } \\
\text { Study Quality: } \\
\text { Fair }\end{array}$ \\
\hline
\end{tabular}


Sexual Dysfunction associated with Second-Generation Antidepressants in Patients with Major Depressive Disorder - Results from a Systematic Review with Network Meta-Analysis. Drug Safety; U. Reichenpfader, G. Gartlehner, L.C. Morgan, A. Greenblatt, B. Nussbaumer, R. A. Hansen, M. Van Noord, L. Lux, and B. N. Gaynes. Corresponding author: U. Reichenpfader, Department for Evidence-based Medicine and Clinical Epidemiology, Krems, Austria; Department of Medical and Health Sciences - Division of Community Medicine, Linköping University, Sweden; ureichenpfader@hotmail.com

\begin{tabular}{|c|c|c|c|c|c|c|}
\hline $\begin{array}{c}\text { Study } \\
\text { Characteristics }\end{array}$ & $\begin{array}{l}\text { Research Objective } \\
\text { Study Design }\end{array}$ & Inclusion/Exclusion & $\begin{array}{c}\text { Baseline } \\
\text { Characteristics }\end{array}$ & $\begin{array}{l}\text { Sexual Dysfunction } \\
\text { (SD) Outcomes/ } \\
\text { Adverse Events } \\
\end{array}$ & $\begin{array}{l}\text { Method used to } \\
\text { determine SD }\end{array}$ & $\begin{array}{c}\text { Analysis and Study } \\
\text { Quality (Risk of } \\
\text { Bias) } \\
\end{array}$ \\
\hline $\begin{array}{l}\text { Author: } \\
\text { Moore et al., } \\
\text { 2005[47] }\end{array}$ & $\begin{array}{l}\text { Research objective: } \\
\text { Efficacy of ESC vs. CIT } \\
\text { in outpatients }\end{array}$ & \multirow{3}{*}{$\begin{array}{l}\text { Inclusion criteria: } \\
\text { - Adults } 18 \text { to } 65 \\
\text { - Diagnosed with } \\
\text { MDD according to } \\
\text { DSM-III or -IV } \\
\text { - MADRS of at least } \\
30\end{array}$} & $\begin{array}{l}\text { Mean age (yrs): } \\
\text { D1: } 44.1 \\
\text { D2: } 46.2\end{array}$ & \multirow{4}{*}{$\begin{array}{l}\text { Sexual dysfunction, } \\
\%: \\
\text { D1: } 0 \\
\text { D2: } 0.7 \\
\text { Overall adverse } \\
\text { events: } \\
\text { D1: } 14.8 \\
\text { D2: } 16.4\end{array}$} & NR & $\begin{array}{l}\text { Overall attrition } \\
\text { rate: } \\
7.5 \%\end{array}$ \\
\hline \multirow{6}{*}{$\begin{array}{l}\text { Country and } \\
\text { setting: } \\
\text { France } \\
\text { Psychiatric and } \\
\text { general practice } \\
\text { Funding: } \\
\text { H. Lundbeck A/S }\end{array}$} & $\begin{array}{l}\text { Duration of study: } \\
8 \mathrm{wks}\end{array}$ & & \multirow{2}{*}{$\begin{array}{l}\text { Sex (\% female): } \\
\text { D1: } 81.7 \\
\text { D2: } 72\end{array}$} & & & $\begin{array}{l}\text { ITT analysis: } \\
\text { Yes }\end{array}$ \\
\hline & \multirow{2}{*}{$\begin{array}{l}\text { Study design: } \\
\text { RCT }\end{array}$} & & & & & Study Quality: \\
\hline & & \multirow{4}{*}{$\begin{array}{l}\text { Exclusion criteria: } \\
\text { - Concomitant } \\
\text { psychotheraputic or } \\
\text { psychotropic } \\
\text { medications } \\
\text { - Additional mental } \\
\text { illnesses or organic } \\
\text { mental disorder } \\
\text { - Illicit drug and } \\
\text { alcohol abuse }\end{array}$} & Race (\% white): & & & Fair \\
\hline & $\begin{array}{l}\text { Overall study N: } \\
294(\text { ITT = 280) }\end{array}$ & & Baseline (HAM-A): & & & \\
\hline & Intervention: & & & & & \\
\hline & $\begin{array}{l}\text { D1: ESC: } 20 \mathrm{mg} / \mathrm{d} \\
\text { D2: CIT: } 40 \mathrm{mg} / \mathrm{d}\end{array}$ & & $\begin{array}{l}\text { Mean HAM-D score } \\
\text { at baseline: } \\
\text { NR }\end{array}$ & & & \\
\hline
\end{tabular}


Sexual Dysfunction associated with Second-Generation Antidepressants in Patients with Major Depressive Disorder - Results from a Systematic Review with Network Meta-Analysis. Drug Safety; U. Reichenpfader, G. Gartlehner, L.C. Morgan, A. Greenblatt, B. Nussbaumer, R. A. Hansen, M. Van Noord, L. Lux, and B. N. Gaynes. Corresponding author: U. Reichenpfader, Department for Evidence-based Medicine and Clinical Epidemiology, Krems, Austria; Department of Medical and Health Sciences - Division of Community Medicine, Linköping University, Sweden; ureichenpfader@hotmail.com

\begin{tabular}{|c|c|c|c|c|c|c|}
\hline $\begin{array}{c}\text { Study } \\
\text { Characteristics }\end{array}$ & $\begin{array}{l}\text { Research Objective } \\
\text { Study Design }\end{array}$ & Inclusion/Exclusion & $\begin{array}{c}\text { Baseline } \\
\text { Characteristics }\end{array}$ & $\begin{array}{l}\text { Sexual Dysfunction } \\
\text { (SD) Outcomes/ } \\
\text { Adverse Events }\end{array}$ & $\begin{array}{l}\text { Method used to } \\
\text { determine SD }\end{array}$ & $\begin{array}{c}\text { Analysis and Study } \\
\text { Quality (Risk of } \\
\text { Bias) }\end{array}$ \\
\hline $\begin{array}{l}\text { Author: } \\
\text { Nemeroff et al., } \\
\text { 1995[48] } \\
\text { Country and } \\
\text { setting: } \\
\text { United States } \\
\text { Multicenter } \\
\text { Funding: } \\
\text { Solvay } \\
\text { Pharmaceuticals }\end{array}$ & $\begin{array}{l}\text { Research objective: } \\
\text { Comparison of efficacy } \\
\text { and safety of FLUV and } \\
\text { SER in treatment of } \\
\text { depression } \\
\text { Duration of study: } \\
7 \text { wks } \\
\text { Study design: } \\
\text { RCT } \\
\text { Overall study } \mathrm{N}: \\
\text { 95 } \\
\text { Intervention: } \\
\text { D1: SER: } 50-200 \mathrm{mg} / \mathrm{d} \\
\text { (137.1) } \\
\text { D2: FLUV: } 50-150 \mathrm{mg} / \mathrm{d} \\
(123.8)\end{array}$ & $\begin{array}{l}\text { Inclusion criteria: } \\
\text { - Adults } 18 \text { to } 65 \\
\text { - Diagnosed with } \\
\text { MDD according to } \\
\text { DSM-III or -IV } \\
\text { - Minimum HAM-D } \\
\text { score of } 20 \\
\text { - HAM-D depressed } \\
\text { mood item of at } \\
\text { least } 2 \\
\text { - Covi anxiety score } \\
\text { less than Raskin } \\
\text { score } \\
\text { - Minimum score of } 8 \\
\text { on Raskin } \\
\text { Depression Scale } \\
\text { Exclusion criteria: } \\
\text { - Pregnant } \\
\text { - Lactating } \\
\text { - Concomitant } \\
\text { psychotheraputic or } \\
\text { psychotropic } \\
\text { medications } \\
\text { - Additional mental } \\
\text { illnesses or organic } \\
\text { mental disorder } \\
\text { - Patients itolerant of } \\
\text { SSRI side effects }\end{array}$ & $\begin{array}{l}\text { Mean age (yrs): } \\
\text { D1: } 41.2 \\
\text { D2: } 38.5 \\
\text { Sex (\% female): } \\
\text { D1: } 60.9 \\
\text { D2: } 61.2 \\
\text { Race (\% white): } \\
\text { D1: } 84.8 \\
\text { D2: } 98.0 \\
\text { Baseline (HAM-A): } \\
\text { NR } \\
\text { Mean HAM-D score } \\
\text { at baseline: } \\
\text { D1: } 23.15 \text { (2.77) } \\
\text { D2: } 24.57 \text { (3.66) }\end{array}$ & $\begin{array}{l}\text { Sexual dysfunction, } \\
\%: \\
\text { D1: } 28 \\
\text { D2: } 10 \\
\text { Overall adverse } \\
\text { events: } \\
\text { D1: } 93.5 \\
\text { D2: } 85.7\end{array}$ & $\begin{array}{l}\text { specific question for } A E \text {, } \\
\text { not described }\end{array}$ & $\begin{array}{l}\text { Overall attrition } \\
\text { rate: } \\
28 \% \\
\text { ITT analysis: } \\
\text { Yes } \\
\text { Study Quality: } \\
\text { Fair }\end{array}$ \\
\hline
\end{tabular}


Sexual Dysfunction associated with Second-Generation Antidepressants in Patients with Major Depressive Disorder - Results from a Systematic Review with Network Meta-Analysis. Drug Safety; U. Reichenpfader, G. Gartlehner, L.C. Morgan, A. Greenblatt, B. Nussbaumer, R. A. Hansen, M. Van Noord, L. Lux, and B. N. Gaynes. Corresponding author: U. Reichenpfader, Department for Evidence-based Medicine and Clinical Epidemiology, Krems, Austria; Department of Medical and Health Sciences - Division of Community Medicine, Linköping University, Sweden; ureichenpfader@hotmail.com

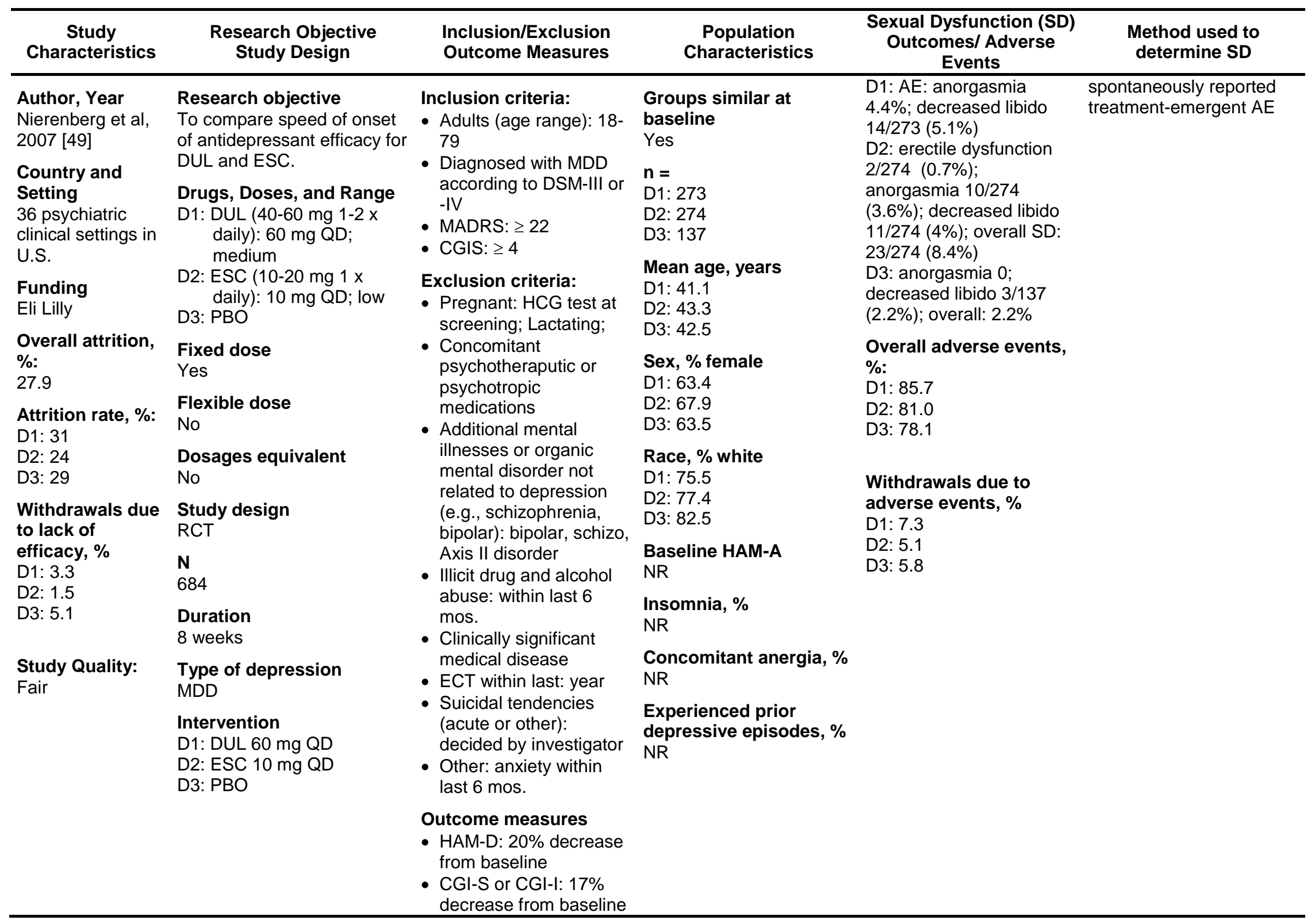


Sexual Dysfunction associated with Second-Generation Antidepressants in Patients with Major Depressive Disorder - Results from a Systematic Review with Network Meta-Analysis. Drug Safety; U. Reichenpfader, G. Gartlehner, L.C. Morgan, A. Greenblatt, B. Nussbaumer, R. A. Hansen, M. Van Noord, L. Lux, and B. N. Gaynes. Corresponding author: U. Reichenpfader, Department for Evidence-based Medicine and Clinical Epidemiology, Krems, Austria; Department of Medical and Health Sciences - Division of Community Medicine, Linköping University, Sweden; ureichenpfader@hotmail.com

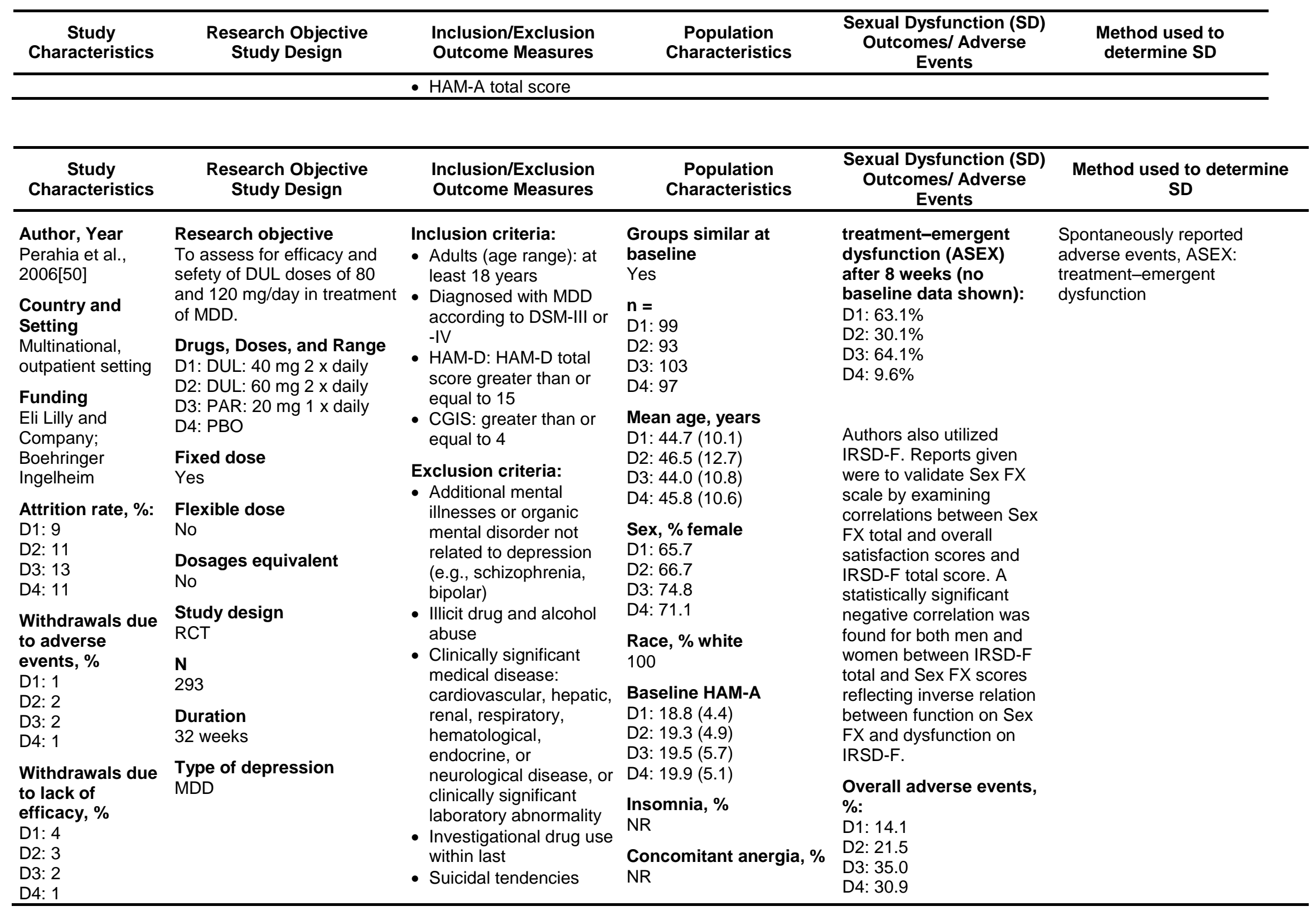


Sexual Dysfunction associated with Second-Generation Antidepressants in Patients with Major Depressive Disorder - Results from a Systematic Review with Network Meta-Analysis. Drug Safety; U. Reichenpfader, G. Gartlehner, L.C. Morgan, A. Greenblatt, B. Nussbaumer, R. A. Hansen, M. Van Noord, L. Lux, and B. N. Gaynes. Corresponding author: U. Reichenpfader, Department for Evidence-based Medicine and Clinical Epidemiology, Krems, Austria; Department of Medical and Health Sciences - Division of Community Medicine, Linköping University, Sweden; ureichenpfader@hotmail.com

\begin{tabular}{|c|c|c|c|c|c|}
\hline $\begin{array}{c}\text { Study } \\
\text { Characteristics }\end{array}$ & $\begin{array}{l}\text { Research Objective } \\
\text { Study Design }\end{array}$ & $\begin{array}{l}\text { Inclusion/Exclusion } \\
\text { Outcome Measures }\end{array}$ & $\begin{array}{c}\text { Population } \\
\text { Characteristics }\end{array}$ & $\begin{array}{c}\text { Sexual Dysfunction (SD) } \\
\text { Outcomes/ Adverse } \\
\text { Events }\end{array}$ & $\begin{array}{l}\text { Method used to determine } \\
\text { SD }\end{array}$ \\
\hline \multirow[t]{2}{*}{$\begin{array}{l}\text { Study Quality: } \\
\text { Fair }\end{array}$} & & $\begin{array}{l}\text { (acute or other) } \\
\text { - Lack of response to at } \\
\text { least two adequate } \\
\text { courses of } \\
\text { antidepressant therapy } \\
\text { (at least } 4 \text { weeks' } \\
\text { duration) within } \\
\text { therapeutic dose range } \\
\text { during their current } \\
\text { MDD episode. }\end{array}$ & $\begin{array}{l}\text { Experienced prior } \\
\text { depressive episodes, \% } \\
\text { NR }\end{array}$ & & \\
\hline & & $\begin{array}{l}\text { Outcome measures } \\
\text { - HAM-D: mean change } \\
\text { from baseline in HAM-D } \\
17 \text { total score after } 8 \\
\text { weeks of treatment } \\
\text { - MADRS } \\
\text { - CGI-S } \\
\text { - PGI scale } \\
\text { - SDS } \\
\text { - VAS) for pain } \\
\text { - SSI }\end{array}$ & & & \\
\hline
\end{tabular}


Sexual Dysfunction associated with Second-Generation Antidepressants in Patients with Major Depressive Disorder - Results from a Systematic Review with Network Meta-Analysis. Drug Safety; U. Reichenpfader, G. Gartlehner, L.C. Morgan, A. Greenblatt, B. Nussbaumer, R. A. Hansen, M. Van Noord, L. Lux, and B. N. Gaynes. Corresponding author: U. Reichenpfader, Department for Evidence-based Medicine and Clinical Epidemiology, Krems, Austria; Department of Medical and Health Sciences - Division of Community Medicine, Linköping University, Sweden; ureichenpfader@hotmail.com

\begin{tabular}{|c|c|c|c|c|c|c|}
\hline $\begin{array}{c}\text { Study } \\
\text { Characteristics }\end{array}$ & $\begin{array}{l}\text { Research Objective } \\
\text { Study Design }\end{array}$ & Inclusion/Exclusion & $\begin{array}{c}\text { Baseline } \\
\text { Characteristics }\end{array}$ & $\begin{array}{l}\text { Sexual Dysfunction } \\
\text { (SD) Outcomes/ } \\
\text { Adverse Events }\end{array}$ & $\begin{array}{l}\text { Method used to } \\
\text { determine SD }\end{array}$ & $\begin{array}{c}\text { Analysis and Study } \\
\text { Quality (Risk of } \\
\text { Bias) }\end{array}$ \\
\hline $\begin{array}{l}\text { Author: } \\
\text { Rabkin et al., } \\
2004[51] \\
\text { Country and } \\
\text { setting: } \\
\text { US } \\
\text { Outpatient } \\
\text { Funding: } \\
\text { Lilly (provided } \\
\text { tablets); } \\
\text { Pharmacia and } \\
\text { Upjohn (provided } \\
\text { coded vials) } \\
\text { National Institute } \\
\text { of Mental Health }\end{array}$ & $\begin{array}{l}\text { Research objective: } \\
\text { To determine whether } \\
\text { testosterone and } \\
\text { FLUOX is superior to } \\
\text { PBO for depression, } \\
\text { fatigue, or both } \\
\text { Duration of study: } \\
8 \text { wks } \\
\text { Study design: } \\
\text { RCT } \\
\text { Overall study N: } \\
\text { 123 } \\
\text { Intervention: } \\
\text { D1: FLUOX: } 20-60 \\
\text { mg/d } \\
\text { D2: PBO Testosterone } \\
200-400 \text { mg biwkly }\end{array}$ & 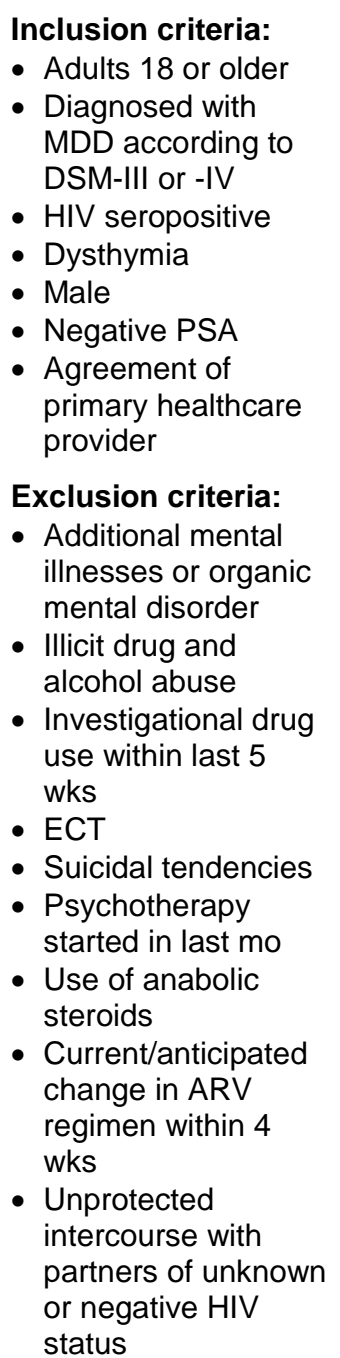 & $\begin{array}{l}\text { Mean age (yrs): } \\
\text { D1: } 40 \\
\text { D2: } 41 \\
\text { Sex (\% female): } \\
\text { D1: } 0 \\
\text { D2: } 0 \\
\text { Race (\% white): } \\
\text { D1: } 21.7 \\
\text { D2: } 23.1 \\
\text { Baseline (HAM-A): } \\
\text { NR } \\
\text { Baseline HAM-D: } \\
\text { D1: } 18.2 \text { (4.5) } \\
\text { D2: } 16.8 \text { (3.3) }\end{array}$ & $\begin{array}{l}\text { Sexual dysfunctional } \\
\text { (male ejaculation): } \\
\text { D1: } 6 \\
\text { D2: } 0\end{array}$ & $\begin{array}{l}\text { Structured Assessment } \\
\text { of Treatment Emergent } \\
\text { Events }\end{array}$ & $\begin{array}{l}\text { Overall attrition } \\
\text { rate: } \\
26.8 \% \\
\text { ITT Analysis } \\
\text { Yes } \\
\text { Study Quality: } \\
\text { Fair }\end{array}$ \\
\hline
\end{tabular}


Sexual Dysfunction associated with Second-Generation Antidepressants in Patients with Major Depressive Disorder - Results from a Systematic Review with Network Meta-Analysis. Drug Safety; U. Reichenpfader, G. Gartlehner, L.C. Morgan, A. Greenblatt, B. Nussbaumer, R. A. Hansen, M. Van Noord, L. Lux, and B. N. Gaynes. Corresponding author: U. Reichenpfader, Department for Evidence-based Medicine and Clinical Epidemiology, Krems, Austria; Department of Medical and Health Sciences - Division of Community Medicine, Linköping University, Sweden; ureichenpfader@hotmail.com

\begin{tabular}{|c|c|c|c|c|c|c|}
\hline $\begin{array}{c}\text { Study } \\
\text { Characteristics }\end{array}$ & $\begin{array}{l}\text { Research Objective } \\
\text { Study Design }\end{array}$ & Inclusion/Exclusion & $\begin{array}{c}\text { Baseline } \\
\text { Characteristics }\end{array}$ & $\begin{array}{l}\text { Sexual Dysfunction } \\
\text { (SD) Outcomes/ } \\
\text { Adverse Events }\end{array}$ & $\begin{array}{l}\text { Method used to } \\
\text { determine SD }\end{array}$ & $\begin{array}{c}\text { Analysis and Study } \\
\text { Quality (Risk of } \\
\text { Bias }\end{array}$ \\
\hline $\begin{array}{l}\text { Author: } \\
\text { Reimherr et } \\
\text { al.,1990 [52] } \\
\text { Country and } \\
\text { setting: } \\
\text { USA, mulitcenter } \\
\text { Funding: } \\
\text { NR }\end{array}$ & $\begin{array}{l}\text { Research objective: } \\
\text { To compare efficacy, } \\
\text { safety, and tolerance of } \\
\text { sertraline against } \\
\text { amitryptyline and } \\
\text { placebo } \\
\text { Duration of study: } \\
8 \text { weeks } \\
\text { Study design: } \\
\text { RCT, double-blind } \\
\text { Overall study N: } \\
\text { 448 } \\
\text { Intervention: } \\
\text { D1: SERTRALINE: } \\
145 \text { mg } \\
\text { D2: PBO }\end{array}$ & $\begin{array}{l}\text { Inclusion criteria: } \\
\text { - Adults } 18 \text { to } 65 \\
\text { - Diagnosed with } \\
\text { MDD according to } \\
\text { DSM-III } \\
\text { - Minimum HAM-D } \\
\text { score of } 18 \text { on the } \\
\text { first } 18 \text { items of the } \\
\text { HAM-D } \\
\text { - less than a 25\% } \\
\text { decrease in HAM_D } \\
\text { score compared } \\
\text { with screening } \\
\text { value; higher score } \\
\text { on the Raskin } \\
\text { Depression Scale } \\
\text { than on Covi } \\
\text { Anxiety Scale } \\
\text { Exclusion criteria: } \\
\text { - Pregnant or } \\
\text { breastfeeding } \\
\text { - Concomitant } \\
\text { psychotheraputic or } \\
\text { psychotropic } \\
\text { medications } \\
\text { - Illicit drug and } \\
\text { alcohol abuse } \\
\text { - significant medical } \\
\text { conditions } \\
\text { - Suicidal tendencies } \\
\text { - Schizophrenia or } \\
\text { schizoaffective } \\
\text { disorder } \\
\end{array}$ & $\begin{array}{l}\text { Mean age (yrs), } \\
\text { women: } \\
\text { D1: } 40.0 \\
\text { D2: } 40.2 \\
\text { Mean age (yrs), men: } \\
\text { D1: } 37.9 \\
\text { D2: } 40.0 \\
\text { Sex (\% female): } \\
\text { D1: } 53 \\
\text { D2: } 52 \\
\text { Race (\% white): } \\
\text { D1: } 91.3 \\
\text { D2: } 92.7 \\
\text { Baseline (HAM-D): } \\
\text { NR }\end{array}$ & $\begin{array}{l}\text { D1: ejaculation } \\
\text { disorder } 15 / 70(21.4 \% \\
\text { of men) } \\
\text { D2: ejaculation } \\
\text { disorder } 1 / 72(1.4 \% \text { of } \\
\text { men) }\end{array}$ & NR & $\begin{array}{l}\text { Overall attrition } \\
\text { rate: } \\
39.2 \% \\
\text { ITT Analysis } \\
\text { modified ITT (at } \\
\text { least one efficacy } \\
\text { assessment } \\
\text { available and at lest } \\
\text { one study dose } \\
\text { medication) } \\
\text { Study Quality: } \\
\text { Fair }\end{array}$ \\
\hline
\end{tabular}


Sexual Dysfunction associated with Second-Generation Antidepressants in Patients with Major Depressive Disorder - Results from a Systematic Review with Network Meta-Analysis. Drug Safety; U. Reichenpfader, G. Gartlehner, L.C. Morgan, A. Greenblatt, B. Nussbaumer, R. A. Hansen, M. Van Noord, L. Lux, and B. N. Gaynes. Corresponding author: U. Reichenpfader, Department for Evidence-based Medicine and Clinical Epidemiology, Krems, Austria; Department of Medical and Health Sciences - Division of Community Medicine, Linköping University, Sweden; ureichenpfader@hotmail.com

\begin{tabular}{|c|c|c|c|c|c|c|}
\hline $\begin{array}{c}\text { Study } \\
\text { Characteristics }\end{array}$ & $\begin{array}{l}\text { Research Objective } \\
\text { Study Design }\end{array}$ & Inclusion/Exclusion & $\begin{array}{c}\text { Baseline } \\
\text { Characteristics }\end{array}$ & $\begin{array}{c}\text { Sexual Dysfunction } \\
\text { (SD) Outcomes/ } \\
\text { Adverse Events }\end{array}$ & $\begin{array}{l}\text { Method used to } \\
\text { determine SD }\end{array}$ & $\begin{array}{c}\text { Analysis and Study } \\
\text { Quality (Risk of } \\
\text { Bias) }\end{array}$ \\
\hline $\begin{array}{l}\text { Author: } \\
\text { Rush et al., } 1998 \\
\text { [53] } \\
\text { Country and } \\
\text { setting: } \\
\text { USA, Canada, } \\
\text { multicenter } \\
\text { Funding: } \\
\text { Bristol-Myers } \\
\text { Squibb } \\
\text { Pharmaceuticals; } \\
\text { Sarah and } \\
\text { Charles Seay } \\
\text { Center for } \\
\text { Research into } \\
\text { the Biological } \\
\text { Basis of } \\
\text { Psychiatric } \\
\text { Disorders, the } \\
\text { University of } \\
\text { Texas } \\
\text { Southwestern } \\
\text { Medical Center, } \\
\text { NIMH }\end{array}$ & $\begin{array}{l}\text { Research objective: } \\
\text { To analyze the } \\
\text { objective and } \\
\text { subjective effects of } \\
\text { nefazodone and } \\
\text { fluoxetine on sleep as } \\
\text { well as the timing of } \\
\text { these effects over an 8- } \\
\text { week, acute-phase } \\
\text { treatment period in } \\
\text { patients with MDD. } \\
\text { Duration of study: } \\
8 \text { weeks } \\
\text { Study design: } \\
\text { RCT } \\
\text { Overall study N: } \\
\text { 125 } \\
\text { Intervention: } \\
\text { D1: Nefazodone, up to } \\
200-500 m g / d \\
\text { D2: Fluoxetine up to } \\
40 \text { mg/d }\end{array}$ & $\begin{array}{l}\text { Inclusion criteria: } \\
\text { - Adults } 19 \text { to } 55 \\
\text { - Diagnosed with } \\
\text { MDD according to } \\
\text { DSM-III-R } \\
\text { - Minimum score of } \\
18 \text { on the first } 17 \\
\text { items of the } \\
\text { Hamilton } \\
\text { Depression Rating } \\
\text { Scale (HDRS-17) } \\
\text { Exclusion criteria: } \\
\text { - engaged in } \\
\text { shiftwork; or had } \\
\text { independent } \\
\text { sleep/wake } \\
\text { disorders identified } \\
\text { on } \\
\text { polysomnographs; } \\
\text { - had documented } \\
\text { significant } \\
\text { concurrent general } \\
\text { medical conditions; } \\
\text { - met DSM-III-R } \\
\text { criteria for } \\
\text { psychoactive } \\
\text { substance use } \\
\text { disorder within the } \\
\text { year prior to study. } \\
\text { - Concomitant } \\
\text { psychotheraputic or } \\
\text { psychotropic } \\
\text { medications } \\
\text { - any other major } \\
\text { lifetime DSM-III-R } \\
\text { Axis I disorders } \\
\text { (e.g., organic } \\
\text { mental syndromes, } \\
\text { bipolar, any }\end{array}$ & $\begin{array}{l}\text { Mean age (yrs): } \\
\text { D1: } 46.0 \\
\text { D2: } 37.0 \\
\text { Sex (\% female): } \\
\text { D1: } 59 \\
\text { D2: } 70 \\
\text { Race (\% white): } \\
\text { D1: } 78 \\
\text { D2: } 85 \\
\text { Mean Baseline HAM- } \\
\text { D-17 score: } \\
\text { D1: } 22.9 \\
\text { D2: } 23.3\end{array}$ & $\begin{array}{l}\text { D1: } 0 \\
\text { D2: erectile } \\
\text { dysfunction } 2 / 18 \\
(11 \%)\end{array}$ & spontaneous reports & $\begin{array}{l}\text { Overall attrition } \\
\text { rate: } \\
17 \% \\
\text { ITT Analysis } \\
\text { modified ITT (all } \\
\text { patients randomized } \\
\text { to treatment, } \\
\text { received a dose of } \\
\text { study drug, and had } \\
\text { at least one sleep or } \\
\text { efficacy evaluation } \\
\text { during treatment) } \\
\text { Study Quality: } \\
\text { Fair }\end{array}$ \\
\hline
\end{tabular}


Sexual Dysfunction associated with Second-Generation Antidepressants in Patients with Major Depressive Disorder - Results from a Systematic Review with Network Meta-Analysis. Drug Safety; U. Reichenpfader, G. Gartlehner, L.C. Morgan, A. Greenblatt, B. Nussbaumer, R. A. Hansen, M. Van Noord, L. Lux, and B. N. Gaynes. Corresponding author: U. Reichenpfader, Department for Evidence-based Medicine and Clinical Epidemiology, Krems, Austria; Department of Medical and Health Sciences - Division of Community Medicine, Linköping University, Sweden; ureichenpfader@hotmail.com

\begin{tabular}{ll}
\hline & psychotic, any \\
& eating, panic, or \\
& obsessive- \\
& compulsive \\
- & pregnant, lactating, \\
& or sexually active \\
& women not using an \\
& adequate method of \\
contraception.
\end{tabular}


Sexual Dysfunction associated with Second-Generation Antidepressants in Patients with Major Depressive Disorder - Results from a Systematic Review with Network Meta-Analysis. Drug Safety; U. Reichenpfader, G. Gartlehner, L.C. Morgan, A. Greenblatt, B. Nussbaumer, R. A. Hansen, M. Van Noord, L. Lux, and B. N. Gaynes. Corresponding author: U. Reichenpfader, Department for Evidence-based Medicine and Clinical Epidemiology, Krems, Austria; Department of Medical and Health Sciences - Division of Community Medicine, Linköping University, Sweden; ureichenpfader@hotmail.com

\begin{tabular}{|c|c|c|c|c|c|}
\hline $\begin{array}{c}\text { Study } \\
\text { Characteristics }\end{array}$ & $\begin{array}{l}\text { Research Objective } \\
\text { Study Design }\end{array}$ & Inclusion/Exclusion & $\begin{array}{c}\text { Population } \\
\text { Characteristics }\end{array}$ & $\begin{array}{c}\text { Sexual Dysfunction (SD) } \\
\text { Outcomes/ Adverse } \\
\text { Events }\end{array}$ & $\begin{array}{l}\text { Method used to } \\
\text { determine SD }\end{array}$ \\
\hline $\begin{array}{l}\text { Author, Year } \\
\text { Schatzberg \& } \\
\text { Roose, 2006[54] } \\
\text { Country and } \\
\text { Setting } \\
\text { United States, } \\
\text { Multicenter (21 } \\
\text { university- } \\
\text { affiliated and } \\
\text { private research } \\
\text { clinics) } \\
\text { Funding } \\
\text { Pharmaceutical } \\
\text { company or } \\
\text { other commercial } \\
\text { source (please } \\
\text { list name): Wyeth } \\
\text { Research } \\
\text { Overall rate of } \\
\text { attrition, \% } \\
\text { 30\% } \\
\text { Attrition rate, \% } \\
\text { D1: } 35.6 \\
\text { D2: } 30 \\
\text { D3: } 4 \\
\text { Withdrawals } \\
\text { due to adverse } \\
\text { events, \% } \\
\text { D1: } 27 \\
\text { D2: } 19 \\
\text { D3: 9.4 }\end{array}$ & $\begin{array}{l}\text { Research objective } \\
\text { To compare efficacy of } \\
\text { VEN IR and FLUOX with } \\
\text { PBO in a sample of } \\
\text { patients over age of } 65 \text { with } \\
\text { depression. } \\
\text { Intervention Drugs, } \\
\text { Doses, and Range } \\
\text { D1: VEN 37.5-225 mg/day } \\
\text { (low - high) } \\
\text { D2: FLUOX 20-60 mg/day } \\
\text { low -high) } \\
\text { D3: PBO } \\
\text { Study design } \\
\text { RCT } \\
\text { n } \\
\text { 300 } \\
\text { Duration } \\
8 \text { weeks } \\
\text { Type of depression } \\
\text { Major depressive disorder } \\
\text { unipolar depression (single } \\
\text { or recurrent, nonpsychotic) }\end{array}$ & 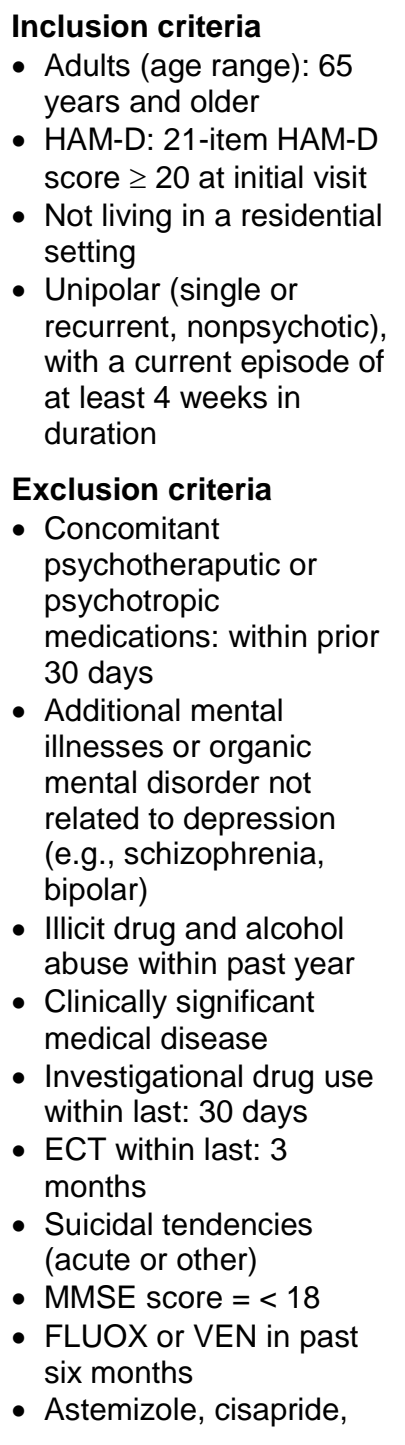 & $\begin{array}{l}\text { Groups similar at } \\
\text { baseline } \\
\text { Yes } \\
\text { n= } \\
\text { D1: } 104 \\
\text { D2: } 100 \\
\text { D3: } 96 \\
\text { Mean age, years } \\
\text { D1: } 71 \\
\text { D2: } 71 \\
\text { D3: } 71 \\
\text { Sex, \% female } \\
\text { D1: } 56 \\
\text { D2: } 45 \\
\text { D3: } 46 \\
\text { Race, \% white } \\
\text { D1: } 93 \\
\text { D2: } 93 \\
\text { D3: } 93 \\
\text { Baseline HAM-A } \\
\text { NR } \\
\text { Insomnia, \%: } \\
\text { NR } \\
\text { Concomitant anergia, \% } \\
\text { NR } \\
\text { Experienced prior } \\
\text { depressive episodes, \% } \\
\text { NR }\end{array}$ & $\begin{array}{l}\text { Sexual dysfunction, \%: } \\
\text { D1: } 8.8 \\
\text { D2: } 8 \\
\text { D3: } 1.0\end{array}$ & NR \\
\hline
\end{tabular}


Sexual Dysfunction associated with Second-Generation Antidepressants in Patients with Major Depressive Disorder - Results from a Systematic Review with Network Meta-Analysis. Drug Safety; U. Reichenpfader, G. Gartlehner, L.C. Morgan, A. Greenblatt, B. Nussbaumer, R. A. Hansen, M. Van Noord, L. Lux, and B. N. Gaynes. Corresponding author: U. Reichenpfader, Department for Evidence-based Medicine and Clinical Epidemiology, Krems, Austria; Department of Medical and Health Sciences - Division of Community Medicine, Linköping University, Sweden; ureichenpfader@hotmail.com

\begin{tabular}{|c|c|c|c|c|c|}
\hline $\begin{array}{c}\text { Study } \\
\text { Characteristics }\end{array}$ & $\begin{array}{l}\text { Research Objective } \\
\text { Study Design }\end{array}$ & Inclusion/Exclusion & $\begin{array}{c}\text { Population } \\
\text { Characteristics }\end{array}$ & $\begin{array}{c}\text { Sexual Dysfunction (SD) } \\
\text { Outcomes/ Adverse } \\
\text { Events }\end{array}$ & $\begin{array}{l}\text { Method used to } \\
\text { determine SD }\end{array}$ \\
\hline $\begin{array}{l}\text { events, \%: } \\
\text { D1: } 26 \\
\text { D2: } 19 \\
\text { D3: } 9.4 \\
\text { Risk of Bias } \\
\text { Fair }\end{array}$ & & $\begin{array}{l}\text { sumatriptan, terfenadine, } \\
\text { PAR, SER, or any } \\
\text { monoamine oxidase } \\
\text { inhibitor within } 14 \text { days } \\
\text { - Used any other } \\
\text { antidepressant, } \\
\text { anxiolytic, or sedative- } \\
\text { hypnotic durg (except } \\
\text { choloral hydrate) } \\
\text { - Known hypersensitivity } \\
\text { to VEN or FLUOX }\end{array}$ & & & \\
\hline
\end{tabular}


Sexual Dysfunction associated with Second-Generation Antidepressants in Patients with Major Depressive Disorder - Results from a Systematic Review with Network Meta-Analysis. Drug Safety; U. Reichenpfader, G. Gartlehner, L.C. Morgan, A. Greenblatt, B. Nussbaumer, R. A. Hansen, M. Van Noord, L. Lux, and B. N. Gaynes. Corresponding author: U. Reichenpfader, Department for Evidence-based Medicine and Clinical Epidemiology, Krems, Austria; Department of Medical and Health Sciences - Division of Community Medicine,

Linköping University, Sweden; ureichenpfader@hotmail.com

\begin{tabular}{|c|c|c|c|c|c|c|}
\hline $\begin{array}{c}\text { Study } \\
\text { Characteristics }\end{array}$ & $\begin{array}{l}\text { Research Objective } \\
\text { Study Design }\end{array}$ & Inclusion/Exclusion & $\begin{array}{c}\text { Baseline } \\
\text { Characteristics }\end{array}$ & $\begin{array}{l}\text { Sexual Dysfunction } \\
\text { (SD) Outcomes/ } \\
\text { Adverse Events }\end{array}$ & $\begin{array}{l}\text { Method used to } \\
\text { determine SD }\end{array}$ & $\begin{array}{c}\text { Analysis and Study } \\
\text { Quality (Risk of } \\
\text { Bias) }\end{array}$ \\
\hline $\begin{array}{l}\text { Author: } \\
\text { Segraves et al., } \\
2000[55] \\
\text { Country and } \\
\text { setting: } \\
\text { United States } \\
\text { Multicenter } \\
\text { Funding: } \\
\text { Glaxo Wellcome, } \\
\text { Inc }\end{array}$ & $\begin{array}{l}\text { Research objective: } \\
\text { To compare efficacy } \\
\text { and safety of BUP SR } \\
\text { and SER, and to } \\
\text { determine whether } \\
\text { baseline anxiety } \\
\text { predicts antidepressant } \\
\text { response } \\
\text { Duration of study: } \\
16 \text { wks } \\
\text { Study design: } \\
\text { RCT } \\
\text { Overall study N: } \\
248 \\
\text { Intervention: } \\
\text { D1: BUP: } 100-300 \\
\text { mg/d (mean } 238 \\
\text { mg/d) } \\
\text { D2: SER: } 50-200 \mathrm{mg} / \mathrm{d} \\
\text { (mean } 114 \mathrm{mg} / \mathrm{d} \text { ) }\end{array}$ & $\begin{array}{l}\text { Inclusion criteria: } \\
\text { - Adults } 18 \text { to } 76 \\
\text { - Diagnosed with } \\
\text { MDD according to } \\
\text { DSM-III or -IV } \\
\text { - Minimum HAM-D } \\
\text { score of } 18 \\
\text { - Stable relationship } \\
\text { with normal sexual } \\
\text { functioning } \\
\text { Exclusion criteria: } \\
\text { - Pregnant } \\
\text { - Lactating } \\
\text { - Concomitant } \\
\text { psychotheraputic or } \\
\text { psychotropic } \\
\text { medications } \\
\text { - Additional mental } \\
\text { illnesses or organic } \\
\text { mental disorder } \\
\text { - Suicidal tendencies } \\
\text { - History/current } \\
\text { diagnosis of eating } \\
\text { disorders } \\
\text { - Known } \\
\text { predisposition to } \\
\text { seizures }\end{array}$ & $\begin{array}{l}\text { Mean age (yrs): } \\
\text { D1: } 39 \\
\text { D2: } 40 \\
\text { Sex (\% female): } \\
\text { D1: } 48 \\
\text { D2: } 48 \\
\text { Race (\% white): } \\
\text { D1: } 93 \\
\text { D2: } 94 \\
\text { Baseline (HAM-A): } \\
\text { D1: } 16.6 \text { (5.2) } \\
\text { D2: } 16.6 \text { (5.2) } \\
\text { Mean HAM-D score } \\
\text { at baseline: } \\
\text { D1: } 24.8(4.6) \\
\text { D2: } 24.8(4.6)\end{array}$ & $\begin{array}{l}\text { D1: overall \% of } \\
\text { sexual desire disorder } \\
\text { (baseline): } 54 \% \text {, at the } \\
\text { end of the treatment } \\
\text { phase: } 21 \% \text {; sexual } \\
\text { dysfunction combined } \\
\text { (sexual arousal } \\
\text { disorder, orgasm } \\
\text { dysfunction, or } \\
\text { premature } \\
\text { ejaculation): men } \\
15 \% \text {; women: } 7 \% \text {; } \\
\text { D2: overall \% of } \\
\text { sexual desire disorder } \\
\text { (treatment-emergent) } \\
26 \% \text {, at the end of the } \\
\text { treatment phase: } \\
\text { sexual dysfunction } \\
\text { combined (sexual } \\
\text { arousal disorder: } 16 \% \text {, } \\
\text { orgasm dysfunction: } \\
52 \% \text {, or premature } \\
\text { ejaculation): men } \\
63 \% \text {; women: } 41 \% \text { : } \\
66 / 126 \text { (52.4\%) }\end{array}$ & $\begin{array}{l}\text { standardized screening } \\
\text { for SD, not specified; } \\
\text { patient satisfaction with } \\
\text { overall sexual } \\
\text { functioning using a 6- } \\
\text { point, Likert-scale; } \\
\text { coding of SD using } \\
\text { DSM-IV }\end{array}$ & $\begin{array}{l}\text { Overall attrition } \\
\text { rate: } \\
31.5 \% \\
\text { ITT analysis: } \\
\text { Yes } \\
\text { Study Quality: } \\
\text { Fair }\end{array}$ \\
\hline
\end{tabular}


Sexual Dysfunction associated with Second-Generation Antidepressants in Patients with Major Depressive Disorder - Results from a Systematic Review with Network Meta-Analysis. Drug Safety; U. Reichenpfader, G. Gartlehner, L.C. Morgan, A. Greenblatt, B. Nussbaumer, R. A. Hansen, M. Van Noord, L. Lux, and B. N. Gaynes. Corresponding author: U. Reichenpfader, Department for Evidence-based Medicine and Clinical Epidemiology, Krems, Austria; Department of Medical and Health Sciences - Division of Community Medicine, Linköping University, Sweden; ureichenpfader@hotmail.com

\begin{tabular}{|c|c|c|c|c|c|}
\hline $\begin{array}{c}\text { Study } \\
\text { Characteristics }\end{array}$ & $\begin{array}{l}\text { Research Objective } \\
\text { Study Design }\end{array}$ & $\begin{array}{l}\text { Inclusion/Exclusion } \\
\text { Outcome Measures }\end{array}$ & $\begin{array}{c}\text { Population } \\
\text { Characteristics }\end{array}$ & $\begin{array}{c}\text { Sexual Dysfunction (SD) } \\
\text { Outcomes/ Adverse } \\
\text { Events }\end{array}$ & $\begin{array}{l}\text { Method used to } \\
\text { determine SD }\end{array}$ \\
\hline $\begin{array}{l}\text { Author, Year } \\
\text { Shelton, 2006[56] } \\
\text { Country and } \\
\text { Setting } \\
\text { Eight U.S. sites } \\
\text { (type not reported) } \\
\text { Funding } \\
\text { Pfizer, Inc. } \\
\text { Attrition } \\
\text { Overall attrition, } \\
\% \text { : } \\
20 \\
\text { Attrition rate, \%: } \\
\text { D1: } 23 \\
\text { D2: } 17 \\
\text { Withdrawals due } \\
\text { to adverse } \\
\text { events, \% } \\
\text { D1: } 1 \\
\text { D2: } 4 \\
\text { Withdrawals due } \\
\text { to lack of } \\
\text { efficacy, \% } \\
\text { D1: NR } \\
\text { D2: NR }\end{array}$ & $\begin{array}{l}\text { Research objective } \\
\text { To compare efficacy, safety, } \\
\text { and tolerability of SER and } \\
\text { VEN XR in outpatients with } \\
\text { MDD. } \\
\text { Drugs, Doses, and Range } \\
\text { D1: SER (25-200 mg } 1 \mathrm{x} \\
\quad \text { daily): } 50-150 \mathrm{mg} \text { QD; } \\
\quad \text { Low-Medium; Maximum } \\
\quad \text { dose as tolerated. } \\
\text { D2: VEN XR (75-225 mg } 1 \text { x } \\
\quad \text { daily): } 75-225 \text { mg QD; } \\
\quad \text { Low-High; Maximum } \\
\quad \text { dose as tolerated. } \\
\text { Fixed dose } \\
\text { No } \\
\text { Flexible dose } \\
\text { Yes } \\
\text { Dosages equivalent } \\
\text { No } \\
\text { Study design } \\
\text { RCT } \\
\text { N } \\
160 \\
\text { Duration } \\
8 \text { weeks } \\
\text { Type of depression } \\
\text { MDD } \\
\text { Intervention } \\
\text { SER } \\
\text { VEN XR }\end{array}$ & $\begin{array}{l}\text { Inclusion criteria: } \\
\text { - Adults aged } 18+ \\
\text { - Diagnosed with MDD } \\
\text { according to DSM-III or } \\
\text {-IV: } \\
\text { - Single episode or } \\
\text { recurrent w/o psychotic } \\
\text { features. } \\
\text { - HAM-D: } \geq 18 \text { on HAM- } \\
\text { D17 and } \geq 2 \text { on item } 1 \\
\text { (depressed mood). } \\
\text { Exclusion criteria: } \\
\text { - Pregnant: Positive } \\
\text { pregnancy test } \\
\text { excluded participant. } \\
\text { - Lactating: Concomitant } \\
\text { psychotheraputic or } \\
\text { psychotropic } \\
\text { medications } \\
\text { - Use of an } \\
\text { antidepressant within } 2 \\
\text { weeks of baseline } \\
\text { (4 weeks for FLUOX) } \\
\text { - Use of any } \\
\text { psychotropics within } 1 \\
\text { week of baseline } \\
\text { (except zolpidem or } \\
\text { zopiclone) } \\
\text { - Use of benzodiazepines } \\
\text { taken on a regular, daily } \\
\text { basis within } 4 \text { weeks of } \\
\text { baseline } \\
\text { - Monoamine oxidase } \\
\text { inhibitors within } 14 \text { days } \\
\text { of baseline evaluation. } \\
\text { - Additional mental } \\
\text { illnesses or organic } \\
\text { mental disorder not }\end{array}$ & $\begin{array}{l}\text { Groups similar at } \\
\text { baseline } \\
\text { Yes } \\
\mathbf{n}= \\
\text { D1: } 82 \\
\text { D2: } 78 \\
\text { Mean age, years (SD) } \\
\text { D1: } 41.2 \text { (12.0) } \\
\text { D2: } 37.2 \text { (11.6) } \\
\text { Sex, \% female } \\
\text { D1: } 46 \\
\text { D2: } 61 \\
\text { Race, \% white } \\
\text { D1: } 83 \\
\text { D2: } 84 \\
\text { Baseline HAM-A (SD) } \\
\text { D1: } 15.7 \text { (5.1) } \\
\text { D2: } 16.0 \text { (4.4) } \\
\text { Insomnia, \% } \\
\text { NR } \\
\text { Concomitant anergia, \% } \\
\text { NR } \\
\text { Experienced prior } \\
\text { depressive episodes, \% } \\
\text { D1: } 51 \\
\text { D2: } 52 \\
\text { Comments: } \\
\text { NR } \\
\end{array}$ & $\begin{array}{l}\text { Sexual dysfunction: } \\
\text { D1: } 15 / 82(18.3 \%) \\
\text { D2: } 16 / 78(20.5 \%) \\
\text { Overall adverse events, } \\
\%: \\
\text { D1: } 80 \\
\text { D2: } 79\end{array}$ & $\begin{array}{l}\text { spontaneously reported } \\
\text { by patient or recorded by } \\
\text { investigator Treatment } \\
\text { Emergent Symptom } \\
\text { Scale) }\end{array}$ \\
\hline
\end{tabular}


Sexual Dysfunction associated with Second-Generation Antidepressants in Patients with Major Depressive Disorder - Results from a Systematic Review with Network Meta-Analysis. Drug Safety; U. Reichenpfader, G. Gartlehner, L.C. Morgan, A. Greenblatt, B. Nussbaumer, R. A. Hansen, M. Van Noord, L. Lux, and B. N. Gaynes. Corresponding author: U. Reichenpfader, Department for Evidence-based Medicine and Clinical Epidemiology, Krems, Austria; Department of Medical and Health Sciences - Division of Community Medicine, Linköping University, Sweden; ureichenpfader@hotmail.com

\begin{tabular}{|c|c|c|c|c|c|}
\hline $\begin{array}{c}\text { Study } \\
\text { Characteristics }\end{array}$ & $\begin{array}{l}\text { Research Objective } \\
\text { Study Design }\end{array}$ & $\begin{array}{l}\text { Inclusion/Exclusion } \\
\text { Outcome Measures }\end{array}$ & $\begin{array}{c}\text { Population } \\
\text { Characteristics }\end{array}$ & $\begin{array}{c}\text { Sexual Dysfunction (SD) } \\
\text { Outcomes/ Adverse } \\
\text { Events }\end{array}$ & $\begin{array}{l}\text { Method used to } \\
\text { determine SD }\end{array}$ \\
\hline & & $\begin{array}{l}\text { related to depression } \\
\text { (e.g., schizophrenia, } \\
\text { bipolar) } \\
\text { - Current or past } \\
\text { diagnosis of bipolar } \\
\text { disorder or any } \\
\text { psychotic disorder } \\
\text { - Current diagnosis of } \\
\text { delirium or dementia } \\
\text { - A mental condition } \\
\text { rendering patient } \\
\text { unable to understand } \\
\text { study } \\
\text { - Schizoid, schizotypal, } \\
\text { or borderline } \\
\text { personality disorder. } \\
\text { - Illicit drug and alcohol } \\
\text { abuse } \\
\text { - Alcohol or } \\
\text { Ddependence or abuse } \\
\text { within last } 6 \text { months. } \\
\text { - Clinically significant } \\
\text { medical disease } \\
\text { - Any serious and/or } \\
\text { unstable medical } \\
\text { condition } \\
\text { - Abnormal baseline } \\
\text { laboratory finding } \\
\text { considered indicative of } \\
\text { conditions that might } \\
\text { affect study results } \\
\text { - Impaired hepatic } \\
\text { function } \\
\text { - Eistory of seizure } \\
\text { disorder. } \\
\text { days } \\
\text { Ecthin last: } 90 \text { days } \\
\text { dingin last: } 30 \\
\text { - }\end{array}$ & & & \\
\hline
\end{tabular}


Sexual Dysfunction associated with Second-Generation Antidepressants in Patients with Major Depressive Disorder - Results from a Systematic Review with Network Meta-Analysis. Drug Safety; U. Reichenpfader, G. Gartlehner, L.C. Morgan, A. Greenblatt, B. Nussbaumer, R. A. Hansen, M. Van Noord, L. Lux, and B. N. Gaynes. Corresponding author: U. Reichenpfader, Department for Evidence-based Medicine and Clinical Epidemiology, Krems, Austria; Department of Medical and Health Sciences - Division of Community Medicine, Linköping University, Sweden; ureichenpfader@hotmail.com

\begin{tabular}{|c|c|c|c|c|c|}
\hline $\begin{array}{c}\text { Study } \\
\text { Characteristics }\end{array}$ & $\begin{array}{l}\text { Research Objective } \\
\text { Study Design }\end{array}$ & $\begin{array}{l}\text { Inclusion/Exclusion } \\
\text { Outcome Measures }\end{array}$ & $\begin{array}{c}\text { Population } \\
\text { Characteristics }\end{array}$ & $\begin{array}{c}\text { Sexual Dysfunction (SD) } \\
\text { Outcomes/ Adverse } \\
\text { Events }\end{array}$ & $\begin{array}{l}\text { Method used to } \\
\text { determine SD }\end{array}$ \\
\hline & & $\begin{array}{l}\text { - Suicidal tendencies } \\
\text { (acute or other): Score } \\
\text { of } 3 \text { or } 4 \text { on suicide item } \\
\text { of HAMD. } \\
\text { - Previous non-response } \\
\text { to SER, VEN XR, or to } \\
2 \text { antidepressants in } \\
\text { current episode } \\
\text { - Use of herbal and/or } \\
\text { homeopathic remedies } \\
\text { within } 2 \text { weeks of } \\
\text { baseline } \\
\text { - History of intolerance or } \\
\text { hypersensitivity to SER } \\
\text { and/or VEN XR } \\
\text { - Likelihood of requiring } \\
\text { treatment during study } \\
\text { period with drugs not } \\
\text { permitted by study } \\
\text { protocol. }\end{array}$ & & & \\
\hline & & $\begin{array}{l}\text { Outcome measures } \\
\text { - HAM-D } \\
\text { - CGI-S and CGI-I } \\
\text { - QOL scales: Q-LES-Q } \\
\text { - HAM-A }\end{array}$ & & & \\
\hline
\end{tabular}


Sexual Dysfunction associated with Second-Generation Antidepressants in Patients with Major Depressive Disorder - Results from a Systematic Review with Network Meta-Analysis. Drug Safety; U. Reichenpfader, G. Gartlehner, L.C. Morgan, A. Greenblatt, B. Nussbaumer, R. A. Hansen, M. Van Noord, L. Lux, and B. N. Gaynes. Corresponding author: U. Reichenpfader, Department for Evidence-based Medicine and Clinical Epidemiology, Krems, Austria; Department of Medical and Health Sciences - Division of Community Medicine,

Linköping University, Sweden; ureichenpfader@hotmail.com

\begin{tabular}{|c|c|c|c|c|c|c|}
\hline $\begin{array}{c}\text { Study } \\
\text { Characteristics }\end{array}$ & $\begin{array}{l}\text { Research Objective } \\
\text { Study Design }\end{array}$ & Inclusion/Exclusion & $\begin{array}{c}\text { Baseline } \\
\text { Characteristics }\end{array}$ & $\begin{array}{l}\text { Sexual Dysfunction } \\
\text { (SD) Outcomes/ } \\
\text { Adverse Events }\end{array}$ & $\begin{array}{l}\text { Method used to } \\
\text { determine SD }\end{array}$ & $\begin{array}{c}\text { Analysis and Study } \\
\text { Quality (Risk of } \\
\text { Bias) }\end{array}$ \\
\hline $\begin{array}{l}\text { Author: } \\
\text { Simon et. al., } \\
\text { 2004[57] } \\
\text { Country and } \\
\text { setting: } \\
\text { United States } \\
\text { Multicenter study } \\
\text { Funding: } \\
\text { Wyeth }\end{array}$ & $\begin{array}{l}\text { Research objective: } \\
\text { To evaluate efficacy of } \\
\text { VEN XR in prevention } \\
\text { of relapse of } \\
\text { depression by } \\
\text { continuation treatment } \\
\text { Duration of study: } \\
8 \text { wk acute phase; } 6 \text { mo } \\
\text { continuation phase } \\
\text { Study design: } \\
\text { RCT } \\
\text { Overall study N: } \\
\text { 318 entered relapse } \\
\text { prevention study (490 } \\
\text { in acute phase) } \\
\text { Intervention: } \\
\text { D1: VEN XR } 75-225 \\
\quad \text { mg/d } \\
\text { D2: PBO }\end{array}$ & $\begin{array}{l}\text { Inclusion criteria: } \\
\text { - Adults } 18+ \\
\text { - Diagnosed with } \\
\text { MDD according to } \\
\text { DSM-III or -IV } \\
\text { - Minimum HAM-D } \\
\text { score of >20 } \\
\text { - No greater than } \\
20 \% \text { decrease in } \\
\text { HAM D between } \\
\text { evaluations } \\
\text { Exclusion criteria: } \\
\text { - Pregnant } \\
\text { - Lactating } \\
\text { - Concomitant } \\
\text { psychotheraputic or } \\
\text { psychotropic } \\
\text { medications } \\
\text { - Clinically sig } \\
\text { medical disease } \\
\text { - Investigational drug } \\
\text { use } \\
\text { - Suicidial tendencies } \\
\text { - Seizure } \\
\text { - Antipsychotic } \\
\text { medication } \\
\text { - FLUOX within } 30 \\
\text { days }\end{array}$ & $\begin{array}{l}\text { Mean age (yrs): } \\
\text { D1: } 43 \\
\text { D2: } 41 \\
\text { Sex (\% female): } \\
\text { D1: } 102(66 \%) \\
\text { D2: } 86(62 \%) \\
\text { Race (\% white): } \\
\text { NR } \\
\text { Baseline HAM-A: } \\
\text { N/A } \\
\text { Baseline HAM-D: } \\
\text { D1: } 6.5 \\
\text { D2: } 6.4\end{array}$ & $\begin{array}{l}\text { Sexual dysfunction: } \\
\text { D1: abnormal } \\
\text { ejaculation } 5.3 \% \text { of } \\
\text { men } \\
\text { D2: abnormal } \\
\text { ejaculation } 1.9 \% \text { of } \\
\text { men } \\
\text { Overall adverse } \\
\text { events: } \\
\text { D1: } 97 \% \\
\text { D2: } 93 \%\end{array}$ & NR & $\begin{array}{l}\text { Overall attrition } \\
\text { rate: } \\
62 \% \\
\text { ITT Analysis } \\
\text { Yes } \\
\text { Study Quality: } \\
\text { Fair }\end{array}$ \\
\hline
\end{tabular}


Sexual Dysfunction associated with Second-Generation Antidepressants in Patients with Major Depressive Disorder - Results from a Systematic Review with Network Meta-Analysis. Drug Safety; U. Reichenpfader, G. Gartlehner, L.C. Morgan, A. Greenblatt, B. Nussbaumer, R. A. Hansen, M. Van Noord, L. Lux, and B. N. Gaynes. Corresponding author: U. Reichenpfader, Department for Evidence-based Medicine and Clinical Epidemiology, Krems, Austria; Department of Medical and Health Sciences - Division of Community Medicine, Linköping University, Sweden; ureichenpfader@hotmail.com

\begin{tabular}{|c|c|c|c|c|c|c|}
\hline $\begin{array}{c}\text { Study } \\
\text { Characteristics }\end{array}$ & $\begin{array}{l}\text { Research Objective } \\
\text { Study Design }\end{array}$ & Inclusion/Exclusion & $\begin{array}{c}\text { Baseline } \\
\text { Characteristics }\end{array}$ & $\begin{array}{c}\text { Sexual Dysfunction } \\
\text { (SD) Outcomes/ } \\
\text { Adverse Events } \\
\end{array}$ & $\begin{array}{l}\text { Method used to } \\
\text { determine SD }\end{array}$ & $\begin{array}{c}\text { Analysis and Study } \\
\text { Quality (Risk of } \\
\text { Bias) }\end{array}$ \\
\hline $\begin{array}{l}\text { Author: } \\
\text { Thase et al., } \\
\text { 1997 [58] } \\
\text { Country and } \\
\text { setting: } \\
\text { United States } \\
\text { Multicenter } \\
\text { Funding: } \\
\text { Wyeth Ayerst } \\
\text { Research }\end{array}$ & $\begin{array}{l}\text { Research objective: } \\
\text { To evaluate efficacy } \\
\text { and tolerability of VEN } \\
\text { XR once daily in } \\
\text { outpatients with MDD } \\
\text { Duration of study: } \\
8 \text { wks } \\
\text { Study design: } \\
\text { RCT } \\
\text { Overall study N: } \\
\text { 197 } \\
\text { Intervention: } \\
\text { D1: VENLAFAXINE } \\
\quad \text { XR: } 75-225 \text { mg/d } \\
\text { D2: PBO }\end{array}$ & $\begin{array}{l}\text { Inclusion criteria: } \\
\text { - Adults } 18 \text { and older } \\
\text { - Diagnosed with } \\
\text { MDD according to } \\
\text { DSM-IV with } \\
\text { duration of at least a } \\
\text { month } \\
\text { - Minimum HAM-D } \\
\text { score of } 20 \text { on the } \\
\text { HAM-D-21 and less } \\
\text { than a } 20 \% \\
\text { decrease in HAM-D } \\
\text { score compared } \\
\text { with screening } \\
\text { value; } \\
\text { Exclusion criteria: } \\
\text { - Pregnant or } \\
\text { breastfeeding } \\
\text { - antipsychotic } \\
\text { medications or ECT } \\
\text { within } 30 \text { days prior } \\
\text { to study entry } \\
\text { - fluoxetine within } 21 \\
\text { days prior to study } \\
\text { entry or MAOs } \\
\text { within } 14 \text { days prior } \\
\text { to study entry } \\
\text { - Illicit drug and } \\
\text { alcohol abuse } \\
\text { - significant medical } \\
\text { conditions } \\
\text { - acute suicidal } \\
\text { tendencies } \\
\text { - history of seizures } \\
\text { - history oof bipolar or } \\
\text { psychotic disorder }\end{array}$ & $\begin{array}{l}\text { Mean age (yrs): } \\
\text { D1: } 40 \\
\text { D2: } 42 \\
\text { Sex (\% female): } \\
\text { D1: } 60 \\
\text { D2: } 61 \\
\text { Race (\% white): } \\
\text { NR } \\
\text { Mean HAM-D-21 } \\
\text { score at baseline: } \\
\text { D1: } 25 \\
\text { D2: } 24 \\
\text { Mean MADRS score } \\
\text { at baseline: } \\
\text { D1: } 28 \\
\text { D2: } 28\end{array}$ & $\begin{array}{l}\text { D1: abnormal } \\
\text { ejaculation/orgasm: } \\
\text { men: } 8 / 35 \text { ( } 22.9 \% \text { of } \\
\text { men); women: } 2 / 60 \\
\text { (3.3\% of women); } \\
\text { impotence } 5 / 35 ; \\
\text { female anorgasmia } \\
\text { 4/60; SD AE: overall: } \\
\text { 19/95 (20\%) } \\
\text { D2: } 2 / 102(2 \%)\end{array}$ & spontaneously reported & $\begin{array}{l}\text { Overall attrition } \\
\text { rate: } \\
34 \% \\
\text { ITT analysis: } \\
\text { modified ITT (at } \\
\text { least received one } \\
\text { dose of study } \\
\text { medication and one } \\
\text { efficacy } \\
\text { assessment) } \\
\text { Study Quality: } \\
\text { Fair }\end{array}$ \\
\hline
\end{tabular}


Sexual Dysfunction associated with Second-Generation Antidepressants in Patients with Major Depressive Disorder - Results from a Systematic Review with Network Meta-Analysis. Drug Safety; U. Reichenpfader, G. Gartlehner, L.C. Morgan, A. Greenblatt, B. Nussbaumer, R. A. Hansen, M. Van Noord, L. Lux, and B. N. Gaynes. Corresponding author: U. Reichenpfader, Department for Evidence-based Medicine and Clinical Epidemiology, Krems, Austria; Department of Medical and Health Sciences - Division of Community Medicine, Linköping University, Sweden; ureichenpfader@hotmail.com

\begin{tabular}{|c|c|c|c|c|c|c|}
\hline $\begin{array}{c}\text { Study } \\
\text { Characteristics }\end{array}$ & $\begin{array}{l}\text { Research Objective } \\
\text { Study Design }\end{array}$ & Inclusion/Exclusion & $\begin{array}{c}\text { Baseline } \\
\text { Characteristics }\end{array}$ & $\begin{array}{l}\text { Sexual Dysfunction } \\
\text { (SD) Outcomes/ } \\
\text { Adverse Events }\end{array}$ & $\begin{array}{l}\text { Method used to } \\
\text { determine SD }\end{array}$ & $\begin{array}{c}\text { Analysis and Study } \\
\text { Quality (Risk of } \\
\text { Bias) }\end{array}$ \\
\hline $\begin{array}{l}\text { Author: } \\
\text { Trivedi et al, } \\
2004 \text { [59] } \\
\text { Country and } \\
\text { setting: } \\
\text { USA, Multicenter } \\
\text { (academic and } \\
\text { community } \\
\text { centers) } \\
\text { Funding: } \\
\text { GlaxoSmithKline } \\
\text { Pharmaceuticals }\end{array}$ & $\begin{array}{l}\text { Research objective: } \\
\text { To evaluate efficacy } \\
\text { and tolerability of lower } \\
\text { doses of paroxetine CR } \\
\text { in patients with MDD } \\
\text { Duration of study: } \\
8 \text { wks } \\
\text { Study design: } \\
\text { RCT, double-blind } \\
\text { Overall study N: } \\
459 \\
\text { Intervention: } \\
\text { D1: PBO } \\
\text { D2: Paroxetine CR } \\
\quad 12.5 \mathrm{mg} / \mathrm{d} \\
\text { D3: Paroxetine CR } \\
25 \mathrm{mg} / \mathrm{d}\end{array}$ & $\begin{array}{l}\text { Inclusion criteria: } \\
\text { - Adults } 18 \text { and older } \\
\text { - Diagnosed with } \\
\text { MDD according to } \\
\text { DSM-IV with } \\
\text { duration of at least a } \\
\text { month } \\
\text { - Minimum HAM-D } \\
\text { score of } 20 \text { on the } \\
\text { HAM-D-17 and a } \\
\text { score of more than } \\
2 \text { on item } 2 \text { on } \\
\text { HAM-D at both } \\
\text { screening and } \\
\text { baseline visit } \\
\text { Exclusion criteria: } \\
\text { - any current } \\
\text { psychiatric disorder } \\
\text { other than MDD } \\
\text { - Concomitant } \\
\text { psychotheraputic or } \\
\text { psychotropic } \\
\text { medications } \\
\text { - lifetime history of a } \\
\text { manic or hypomanic } \\
\text { episode, } \\
\text { schizophrenia, } \\
\text { schozoaffective } \\
\text { disorder or bipolar } \\
\text { disorder } \\
\text { - general medical } \\
\text { condition, any } \\
\text { - substance abuse } \\
\text { disorder within the } \\
\text { previous } 3 \text { months, } \\
\text { - substance } \\
\text { dependence within } \\
\text { the previous } 6 \\
\text { months } \\
\text { and }\end{array}$ & $\begin{array}{l}\text { Groups similar at } \\
\text { baseline: } \\
\text { yes } \\
\text { Mean age (yrs): } \\
\text { D1: } 38.4 \\
\text { D2: } 38.6 \\
\text { D3: } 39.4 \\
\text { Sex (\% female): } \\
\text { D1: } 61.6 \\
\text { D2: } 54.2 \\
\text { D3: } 59.5 \\
\text { Race (\% white): } \\
\text { D1: } 74.0 \\
\text { D2: } 76.5 \\
\text { D3: } 76.4 \\
\text { Mean (HAMD-27) } \\
\text { score at baseline: } \\
\text { D1: } 23.8 \\
\text { D2: } 23.2 \\
\text { D3: } 23.5 \\
\text { Mean CGI-S score at } \\
\text { baseline: } \\
\text { D1: } 3.0 \\
\text { D2: } 3.0 \\
\text { D3: } 3.0\end{array}$ & $\begin{array}{l}\text { D1: men \& women, } \\
\text { decrease2 libido: } \\
\text { 6/146 (4.2\%) } \\
\text { D2: men \& women, } \\
\text { decreased libido: } \\
\text { 12/156 (7.7\%); } \\
\text { ejaculation disorder: } \\
\text { 4/70 (5.7\% of men); } \\
\text { D3: men \& women, } \\
\text { decreased libido: } \\
\text { 4/154 (2.6\%); } \\
\text { ejaculation disorder: } \\
\text { 6/70 (8.6\% of men) }\end{array}$ & $\begin{array}{l}\text { patients questioned at } \\
\text { each visit to report } A E\end{array}$ & $\begin{array}{l}\text { Overall attrition } \\
\text { rate: } \\
23.7 \% \\
\text { ITT analysis: } \\
\text { modified intent-to- } \\
\text { treat set comprising } \\
\text { all patients who had } \\
\text { at least one valid } \\
\text { postbaseline } \\
\text { assessment of the } \\
\text { primary efficacy } \\
\text { variable and } \\
\text { received at least one } \\
\text { dose of study } \\
\text { medication } \\
\text { Study Quality: } \\
\text { fair }\end{array}$ \\
\hline
\end{tabular}


Sexual Dysfunction associated with Second-Generation Antidepressants in Patients with Major Depressive Disorder - Results from a Systematic Review with Network Meta-Analysis. Drug Safety; U. Reichenpfader, G. Gartlehner, L.C. Morgan, A. Greenblatt, B. Nussbaumer, R. A. Hansen, M. Van Noord, L. Lux, and B. N. Gaynes. Corresponding author: U. Reichenpfader, Department for Evidence-based Medicine and Clinical Epidemiology, Krems, Austria; Department of Medical and Health Sciences - Division of Community Medicine, Linköping University, Sweden; ureichenpfader@hotmail.com

\begin{aligned} & \hline presence or history \\ & of a clinically \\ & significant \\ & neurological \\ &- Suicidal tendencies \\ &- lifetime history of \\ & seizures \\ &$\bullet$ ECT within pevious \\ & 3 months \\ &- use of study \\ & medication within \\ & previous 12 months \\ & \hline\end{aligned}


Sexual Dysfunction associated with Second-Generation Antidepressants in Patients with Major Depressive Disorder - Results from a Systematic Review with Network Meta-Analysis. Drug Safety; U. Reichenpfader, G. Gartlehner, L.C. Morgan, A. Greenblatt, B. Nussbaumer, R. A. Hansen, M. Van Noord, L. Lux, and B. N. Gaynes. Corresponding author: U. Reichenpfader, Department for Evidence-based Medicine and Clinical Epidemiology, Krems, Austria; Department of Medical and Health Sciences - Division of Community Medicine, Linköping University, Sweden; ureichenpfader@hotmail.com

\begin{tabular}{|c|c|c|c|c|c|}
\hline $\begin{array}{c}\text { Study } \\
\text { Characteristics }\end{array}$ & $\begin{array}{l}\text { Research Objective } \\
\text { Study Design }\end{array}$ & $\begin{array}{l}\text { Inclusion/Exclusion } \\
\text { Outcome Measures }\end{array}$ & $\begin{array}{c}\text { Population } \\
\text { Characteristics }\end{array}$ & $\begin{array}{c}\text { Sexual Dysfunction (SD) } \\
\text { Outcomes/ Adverse } \\
\text { Events } \\
\end{array}$ & $\begin{array}{l}\text { Method used to } \\
\text { determine SD }\end{array}$ \\
\hline $\begin{array}{l}\text { Author, Year } \\
\text { Ventura et al, } \\
2007[60] \\
\text { Country and } \\
\text { Setting } \\
\text { multicenter (8 } \\
\text { Centers). United } \\
\text { States } \\
\text { Funding } \\
\text { Forest } \\
\text { Laboratories } \\
\text { Attrition } \\
\text { Overall attrition, } \\
\% \text { : } \\
\text { 16\% } \\
\text { Attrition rate, \%: } \\
\text { D1: } 17 \% \\
\text { D2: } 14 \% \\
\text { Withdrawals due } \\
\text { to adverse } \\
\text { events, \% } \\
\text { D1: } 2 \\
\text { D2: } 4\end{array}$ & $\begin{array}{l}\text { Research objective } \\
\text { Comparison of efficacy and } \\
\text { tolerability of a fixed dose of } \\
\text { ESC with SER } \\
\text { Drugs, Doses, and Range } \\
\text { D1: ESC (10-20 mg } 1 \mathrm{x} \\
\quad \text { daily): } 10 \mathrm{mg} \text { QD; Low } \\
\text { D2: SER (25-200 mg } 1 \mathrm{x} \\
\quad \text { daily): } 50-200 \mathrm{mg} \text { QD; } \\
\quad \text { Low, Medium, or High } \\
\text { Fixed dose } \\
\text { No } \\
\text { Flexible dose } \\
\text { Yes } \\
\text { Dosages equivalent } \\
\text { No } \\
\text { Study design } \\
\text { RCT } \\
\text { N } \\
215 \\
\text { Duration } \\
8 \text { week + } 1 \text { week lead-in } \\
\text { Type of depression } \\
\text { MDD } \\
\text { Intervention } \\
\text { ESC } \\
\text { SER }\end{array}$ & $\begin{array}{l}\text { Inclusion criteria: } \\
\text { - Adults (age range): 18- } \\
80 \\
\text { - Diagnosed with MDD } \\
\text { according to DSM-III or } \\
\text {-IV: DSM-IV } \\
\text { - MADRS: ¡Ý22 at both } \\
\text { screening and baseline } \\
\text { - CGIS: Concomitant } \\
\text { condition (e.g., } \\
\text { alcoholism, anxiety, } \\
\text { stroke) } \\
\text { Exclusion criteria: } \\
\text { - Pregnant } \\
\text { - Negative pregnancy } \\
\text { test } \\
\text { - Women of childbearing } \\
\text { potential not on } \\
\text { accepted form of } \\
\text { contraception } \\
\text { - Lactating } \\
\text { - Concomitant } \\
\text { psychotheraputic or } \\
\text { psychotropic } \\
\text { medications } \\
\text { - Use of a depot } \\
\text { neruoleptic within } 6 \\
\text { months. } \\
\text { - Use of any neuroleptic, } \\
\text { antidepressant, or } \\
\text { anxiolytic medication } \\
\text { within } 2 \text { week (5 weeks } \\
\text { for FLUOX). } \\
\text { - Treatment with either } \\
\text { ESCalopam or SER. } \\
\text { - Failure to respond to } \\
\text { adequate trials of any } \\
\text { two SSRIs. } \\
\text { - Any psychotropic } \\
\text { - Ant }\end{array}$ & $\begin{array}{l}\text { Groups similar at } \\
\text { baseline } \\
\text { Yes } \\
\mathbf{n}= \\
\text { D1: } 107 \\
\text { D2: } 108 \\
\text { Mean age, years } \\
\text { D1: } 40.6 \\
\text { D2: } 38.1 \\
\text { Sex, \% female } \\
\text { D1: } 54.8 \% \\
\text { D2: } 60.2 \% \\
\text { Race, \% white } \\
\text { D1: } 82.7 \% \\
\text { D2: } 89.8 \% \\
\text { Baseline HAM-A } \\
\text { D1: } 15.9 \text { (0.5 SE) } \\
\text { D2: } 15.6 \text { (0.5 SE) } \\
\text { Insomnia, \% } \\
\text { NR } \\
\text { Concomitant anergia, \% } \\
\text { NR } \\
\text { Experienced prior } \\
\text { depressive episodes, \% } \\
\text { NR } \\
\text { Comments: } \\
\text { Use of sleep medication, } \\
\% \\
\text { D1: } 9.6 \\
\text { D2: } 7.4\end{array}$ & $\begin{array}{l}\text { Sexual dysfunction: } \\
\text { D1: Ejaculation disorder: } \\
11 / 47 \text { ( } 23.4 \% \text { of } \\
\text { men); Libido } \\
\text { decreased: } 10 / 107 \\
(9.3 \% \\
\text { D2: Ejaculation disorder: } \\
\text { 10/43 (23.3\%); Libido } \\
\text { decreased: } 15 / 108 \\
(13.9 \%)\end{array}$ & spontaneously reported \\
\hline
\end{tabular}


Sexual Dysfunction associated with Second-Generation Antidepressants in Patients with Major Depressive Disorder - Results from a Systematic Review with Network Meta-Analysis. Drug Safety; U. Reichenpfader, G. Gartlehner, L.C. Morgan, A. Greenblatt, B. Nussbaumer, R. A. Hansen, M. Van Noord, L. Lux, and B. N. Gaynes. Corresponding author: U. Reichenpfader, Department for Evidence-based Medicine and Clinical Epidemiology, Krems, Austria; Department of Medical and Health Sciences - Division of Community Medicine, Linköping University, Sweden; ureichenpfader@hotmail.com

\begin{tabular}{|c|c|c|c|c|c|}
\hline $\begin{array}{c}\text { Study } \\
\text { Characteristics }\end{array}$ & $\begin{array}{l}\text { Research Objective } \\
\text { Study Design }\end{array}$ & $\begin{array}{l}\text { Inclusion/Exclusion } \\
\text { Outcome Measures }\end{array}$ & $\begin{array}{c}\text { Population } \\
\text { Characteristics }\end{array}$ & $\begin{array}{c}\text { Sexual Dysfunction (SD) } \\
\text { Outcomes/ Adverse } \\
\text { Events }\end{array}$ & $\begin{array}{l}\text { Method used to } \\
\text { determine SD }\end{array}$ \\
\hline & & $\begin{array}{l}\text { Dexcept zaleplon or } \\
\text { zolpidem for sleep. } \\
\text { - Additional mental } \\
\text { illnesses or organic } \\
\text { mental disorder not } \\
\text { related to depression } \\
\text { (e.g., schizophrenia, } \\
\text { bipolar) }\end{array}$ & & & \\
\hline & & $\begin{array}{l}\text { Following were all listed } \\
\text { as exclusion criteria: } \\
\text { - Primary Axis I disorder } \\
\text { other than MDD } \\
\text { - history of any DSM-IV } \\
\text { defined psychotic } \\
\text { disorder } \\
\text { - DSM-IV criteria for } \\
\text { bipolar disorder, } \\
\text { schizophrenia, } \\
\text { obsessive-compulsive } \\
\text { disorder, mental } \\
\text { retardation, or } \\
\text { pervasive development } \\
\text { disorder. } \\
\text { - Current psychotic } \\
\text { disorder, personality } \\
\text { disorder of sufficient } \\
\text { severity to interfere with } \\
\text { participation. } \\
\text { - Illicit drug and alcohol } \\
\text { abuse: Dependency as } \\
\text { defined by DSM-IV. } \\
\text { - Clinically significant } \\
\text { medical disease } \\
\text { - Findings from physical } \\
\text { examination, laboratory } \\
\text { test, and ECG were } \\
\text { required to be normal or } \\
\text { clinically insignificant. } \\
\text { - Investigational drug use } \\
\text { within last month. }\end{array}$ & & & \\
\hline
\end{tabular}


Sexual Dysfunction associated with Second-Generation Antidepressants in Patients with Major Depressive Disorder - Results from a Systematic Review with Network Meta-Analysis. Drug Safety; U. Reichenpfader, G. Gartlehner, L.C. Morgan, A. Greenblatt, B. Nussbaumer, R. A. Hansen, M. Van Noord, L. Lux, and B. N. Gaynes. Corresponding author: U. Reichenpfader, Department for Evidence-based Medicine and Clinical Epidemiology, Krems, Austria; Department of Medical and Health Sciences - Division of Community Medicine, Linköping University, Sweden; ureichenpfader@hotmail.com

\begin{tabular}{|c|c|c|c|c|c|}
\hline $\begin{array}{c}\text { Study } \\
\text { Characteristics }\end{array}$ & $\begin{array}{l}\text { Research Objective } \\
\text { Study Design }\end{array}$ & $\begin{array}{l}\text { Inclusion/Exclusion } \\
\text { Outcome Measures }\end{array}$ & $\begin{array}{c}\text { Population } \\
\text { Characteristics }\end{array}$ & $\begin{array}{c}\text { Sexual Dysfunction (SD) } \\
\text { Outcomes/ Adverse } \\
\text { Events }\end{array}$ & $\begin{array}{l}\text { Method used to } \\
\text { determine SD }\end{array}$ \\
\hline & & $\begin{array}{l}\text { - Suicidal tendencies } \\
\text { (acute or other) }\end{array}$ & & & \\
\hline & & $\begin{array}{l}\text { Outcome measures } \\
\text { - HAM-D: HAMD } \\
\text { baseline; HAMD anxiety } \\
\text { subscale } \\
\text { - MADRS } \\
\text { - CGI-S and CGI-I } \\
\text { - Quality of life scales: } \\
\text { Quality of Life } \\
\text { Enjoyment and } \\
\text { Satisfaction } \\
\text { Questionnaire } \\
\text { - Others: HAM-A; CES-D }\end{array}$ & & & \\
\hline
\end{tabular}


Sexual Dysfunction associated with Second-Generation Antidepressants in Patients with Major Depressive Disorder - Results from a Systematic Review with Network Meta-Analysis. Drug Safety; U. Reichenpfader, G. Gartlehner, L.C. Morgan, A. Greenblatt, B. Nussbaumer, R. A. Hansen, M. Van Noord, L. Lux, and B. N. Gaynes. Corresponding author: U. Reichenpfader, Department for Evidence-based Medicine and Clinical Epidemiology, Krems, Austria; Department of Medical and Health Sciences - Division of Community Medicine, Linköping University, Sweden; ureichenpfader@hotmail.com

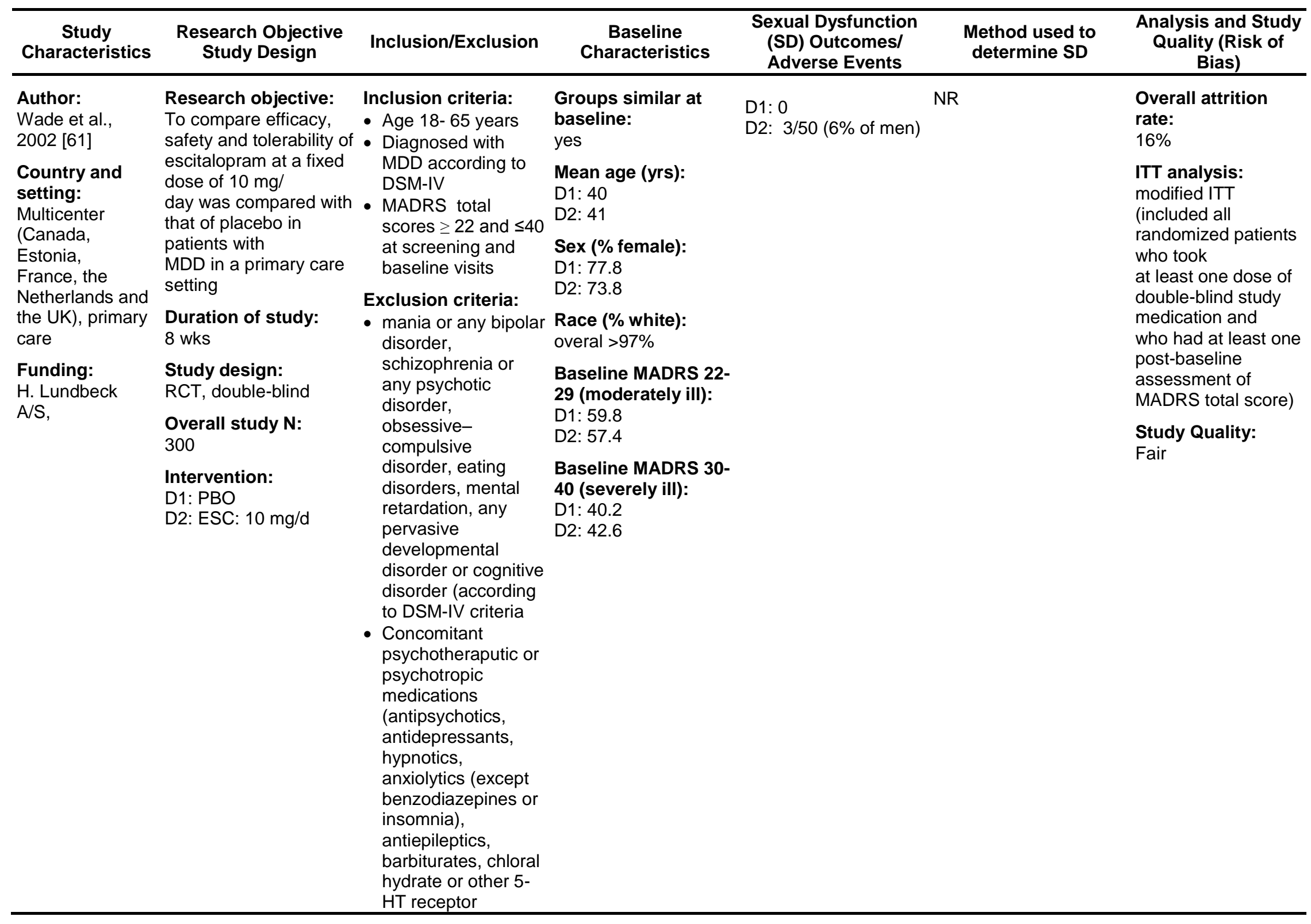


Sexual Dysfunction associated with Second-Generation Antidepressants in Patients with Major Depressive Disorder - Results from a Systematic Review with Network Meta-Analysis. Drug Safety; U. Reichenpfader, G. Gartlehner, L.C. Morgan, A. Greenblatt, B. Nussbaumer, R. A. Hansen, M. Van Noord, L. Lux, and B. N. Gaynes. Corresponding author: U. Reichenpfader, Department for Evidence-based Medicine and Clinical Epidemiology, Krems, Austria; Department of Medical and Health Sciences - Division of Community Medicine, Linköping University, Sweden; ureichenpfader@hotmail.com

agonists

- MADRS score $\geq 5$

on item 10 (suicida

thoughts)

- ECT, treatment with

behaviour therapy

or psychotherapy 
Sexual Dysfunction associated with Second-Generation Antidepressants in Patients with Major Depressive Disorder - Results from a Systematic Review with Network Meta-Analysis. Drug Safety; U. Reichenpfader, G. Gartlehner, L.C. Morgan, A. Greenblatt, B. Nussbaumer, R. A. Hansen, M. Van Noord, L. Lux, and B. N. Gaynes. Corresponding author: U. Reichenpfader, Department for Evidence-based Medicine and Clinical Epidemiology, Krems, Austria; Department of Medical and Health Sciences - Division of Community Medicine, Linköping University, Sweden; ureichenpfader@hotmail.com

\begin{tabular}{|c|c|c|c|c|c|}
\hline $\begin{array}{c}\text { Study } \\
\text { Characteristics }\end{array}$ & $\begin{array}{l}\text { Research Objective } \\
\text { Study Design }\end{array}$ & $\begin{array}{l}\text { Inclusion/Exclusion } \\
\text { Outcome Measures }\end{array}$ & $\begin{array}{c}\text { Population } \\
\text { Characteristics }\end{array}$ & $\begin{array}{c}\text { Sexual Dysfunction (SD) } \\
\text { Outcomes/ Adverse } \\
\text { Events }\end{array}$ & $\begin{array}{l}\text { Method used to } \\
\text { determine SD }\end{array}$ \\
\hline $\begin{array}{l}\text { Author, Year } \\
\text { Wade et al., } \\
2007[62] \\
\text { Country and } \\
\text { Setting } \\
\text { Multinational, } \\
\text { Multicenters } \\
\text { (psychiatric } \\
\text { outpatient and } \\
\text { general practice } \\
\text { settings) } \\
\text { Funding } \\
\text { H. Lundbeck A/S } \\
\text { Attrition } \\
\text { Overall attrition, } \\
\% \text { : } \\
23 \% \\
\text { Attrition rate, \%: } \\
\text { D1: } 22.2 \\
\text { D2: } 24.5 \\
\text { Withdrawals due } \\
\text { to adverse } \\
\text { events, \% } \\
\text { D1: } 9.0 \\
\text { D2: } 17.2 \\
\text { Withdrawals due } \\
\text { to lack of } \\
\text { efficacy, \% } \\
\text { D1: } 4.9 \\
\text { D2: } 1.3\end{array}$ & $\begin{array}{l}\text { Research objective } \\
\text { The objective was to } \\
\text { examine efficacy and } \\
\text { tolerability of ESC compared } \\
\text { to DUL in patients with } \\
\text { moderate to severe MDD } \\
\text { patients over } 24 \text { weeks, with } \\
\text { a secondary enpoint at } 8 \\
\text { weeks. } \\
\text { Drugs, Doses, and Range } \\
\text { D1: ESC } 20 \text { mg/day (Primary } \\
\quad \text { Analysis- endpoint at } 24 \\
\quad \text { weeks) } \\
\text { D2: DUL } 60 \text { mg/day (Primary } \\
\quad \text { Analysis- endpoint at } 24 \\
\quad \text { weeks) } \\
\text { Fixed dose } \\
\text { Yes } \\
\text { Flexible dose } \\
\text { No }\end{array}$ & $\begin{array}{l}\text { Inclusion criteria: } \\
\text { - Adults (age range): } \\
18 \text { - } 65 \text { years of age } \\
\text { - Diagnosed with MDD } \\
\text { according to DSM-III or } \\
\text {-IV } \\
\text { - MADRS: total score } \\
\text { greater than or equal to } \\
\text { 26 } \\
\text { - CGIS: greater than or } \\
\text { equal to } 4 \\
\text { - Other: Patients with a } \\
\text { secondary current } \\
\text { comorbid anxiety } \\
\text { disorder could be } \\
\text { included, except } \\
\text { obsessive-compulsive } \\
\text { disorder, post traumatic } \\
\text { stress disorder, or panic } \\
\text { disorder } \\
\text { Exclusion criteria: } \\
\text { - Pregnant } \\
\text { - Lactating } \\
\text { - Concomitant } \\
\text { psychotheraputic or } \\
\text { psychotropic } \\
\text { medications: (except } \\
\text { zolpidem, zolpiclone } \\
\text { and zaleplon used } \\
\text { episodically for } \\
\text { insomnia) within } 2 \\
\text { weeks prior to baseline } \\
\text { or during study } \\
\text { - Additional mental } \\
\text { illnesses or organic } \\
\text { mental disorder not } \\
\text { related to depression } \\
\text { (e.g., schizophrenia, }\end{array}$ & $\begin{array}{l}\text { Groups similar at } \\
\text { baseline } \\
\text { Yes } \\
\mathbf{n}= \\
\text { D1: } 141 \\
\text { D2: } 146 \\
\text { Mean age, years } \\
\text { D1: } 43.3 \text { (11.6) } \\
\text { D2: } 44.5 \text { (11.0) } \\
\text { Sex, \% female } \\
\text { D1: } 74.1 \\
\text { D2: } 70.2 \\
\text { Race, \% white } \\
\text { D1: } 94.4 \\
\text { D2: } 97.4 \\
\text { Baseline HAM-A } \\
\text { D1: } 22.1 \text { (7.6) } \\
\text { D2: } 21.9 \text { (6.5) } \\
\text { Insomnia, \% } \\
\text { NR } \\
\text { Concomitant anergia, \% } \\
\text { NR } \\
\text { Experienced prior } \\
\text { depressive episodes, \% } \\
\text { NR } \\
\text { Comments: } \\
\text { Base on intent-to-treat } \\
\text { population (ESC, } n=141 \text {, } \\
\text { DUL, } n=146)\end{array}$ & $\begin{array}{l}\text { Sexual dysfunction } \\
\text { (including anorgasmia, } \\
\text { ejaculation failure, erectile } \\
\text { dysfunction, ejaculation } \\
\text { delayed, orgasm } \\
\text { abnormal, ejaculation } \\
\text { disorder, libido decrease } \\
\text { and loss of libido): } \\
\text { D1: } 7 / 144(4.9 \%) \\
\text { D2: } 10 / 151(6.6 \%) \\
\text { Overall adverse events, } \\
\%: \\
\text { D1: } 77.6 \\
\text { D2: } 74.8\end{array}$ & $\begin{array}{l}\text { reported spontaneously } \\
\text { by the patients or in } \\
\text { response to a non-leading } \\
\text { question by the } \\
\text { investigator }\end{array}$ \\
\hline
\end{tabular}


Sexual Dysfunction associated with Second-Generation Antidepressants in Patients with Major Depressive Disorder - Results from a Systematic Review with Network Meta-Analysis. Drug Safety; U. Reichenpfader, G. Gartlehner, L.C. Morgan, A. Greenblatt, B. Nussbaumer, R. A. Hansen, M. Van Noord, L. Lux, and B. N. Gaynes. Corresponding author: U. Reichenpfader, Department for Evidence-based Medicine and Clinical Epidemiology, Krems, Austria; Department of Medical and Health Sciences - Division of Community Medicine, Linköping University, Sweden; ureichenpfader@hotmail.com

\begin{tabular}{|c|c|c|c|c|c|}
\hline $\begin{array}{c}\text { Study } \\
\text { Characteristics }\end{array}$ & $\begin{array}{l}\text { Research Objective } \\
\text { Study Design }\end{array}$ & $\begin{array}{l}\text { Inclusion/Exclusion } \\
\text { Outcome Measures }\end{array}$ & $\begin{array}{c}\text { Population } \\
\text { Characteristics }\end{array}$ & $\begin{array}{c}\text { Sexual Dysfunction (SD) } \\
\text { Outcomes/ Adverse } \\
\text { Events }\end{array}$ & $\begin{array}{l}\text { Method used to } \\
\text { determine SD }\end{array}$ \\
\hline & & 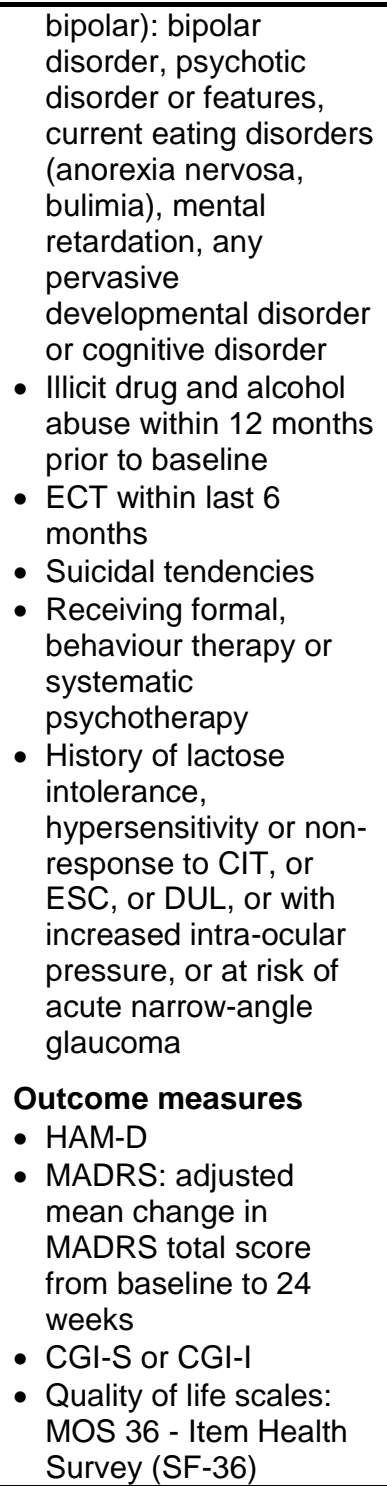 & 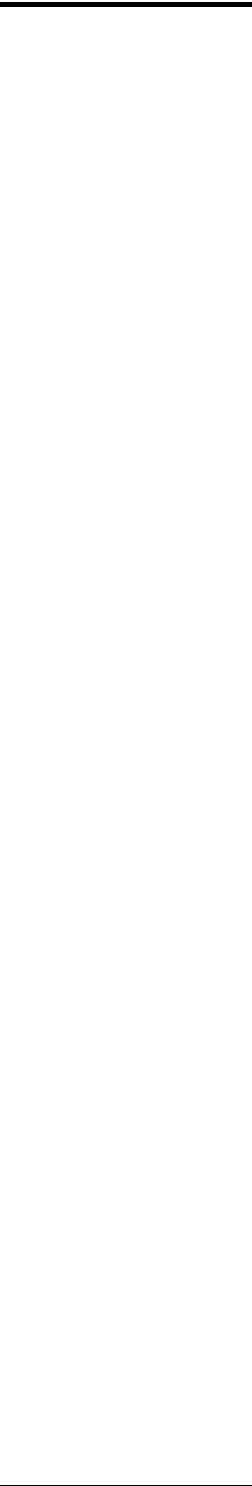 & & \\
\hline
\end{tabular}


Sexual Dysfunction associated with Second-Generation Antidepressants in Patients with Major Depressive Disorder - Results from a Systematic Review with Network Meta-Analysis. Drug Safety; U. Reichenpfader, G. Gartlehner, L.C. Morgan, A. Greenblatt, B. Nussbaumer, R. A. Hansen, M. Van Noord, L. Lux, and B. N. Gaynes. Corresponding author: U. Reichenpfader, Department for Evidence-based Medicine and Clinical Epidemiology, Krems, Austria; Department of Medical and Health Sciences - Division of Community Medicine, Linköping University, Sweden; ureichenpfader@hotmail.com

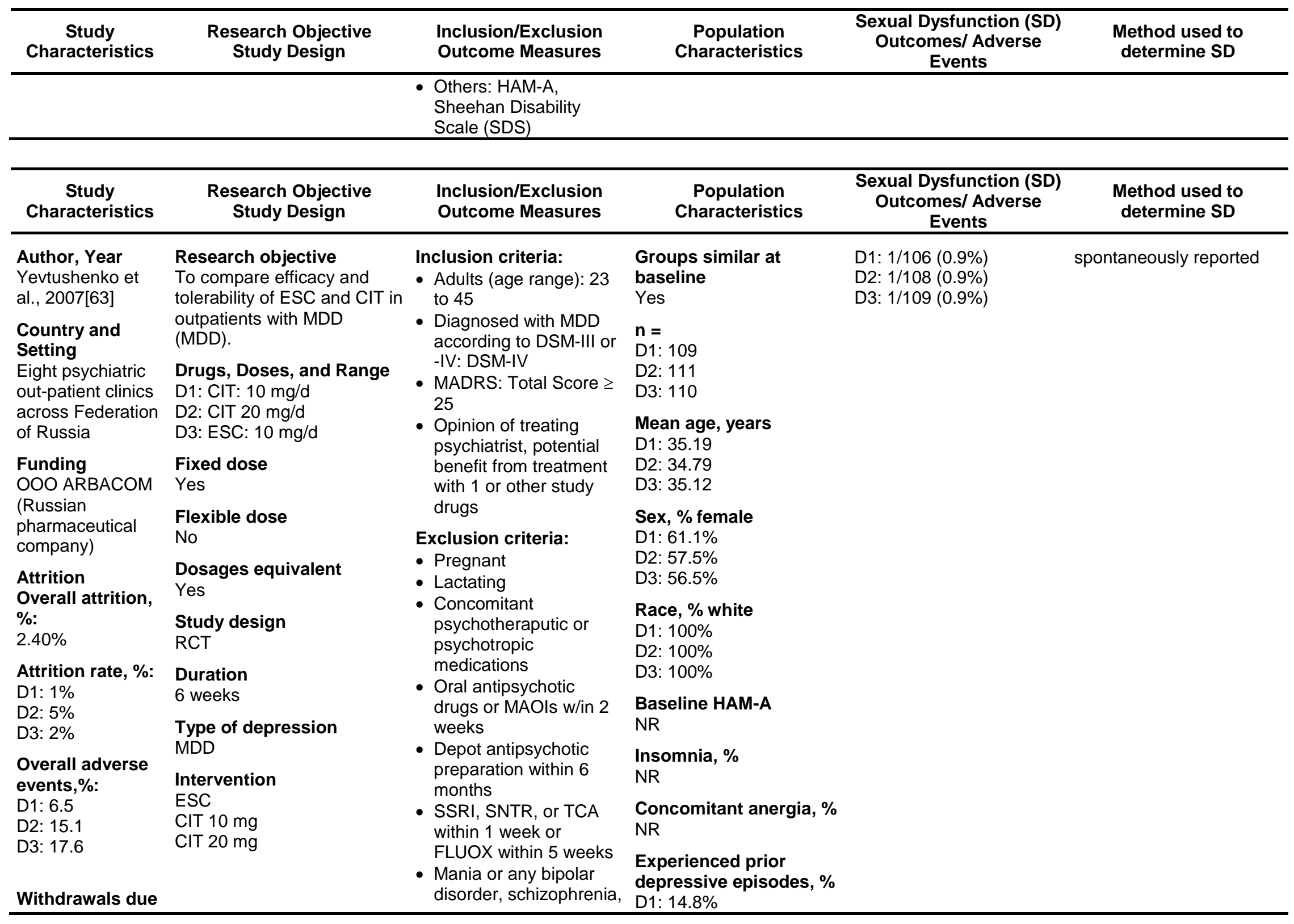


Sexual Dysfunction associated with Second-Generation Antidepressants in Patients with Major Depressive Disorder - Results from a Systematic Review with Network Meta-Analysis. Drug Safety; U. Reichenpfader, G. Gartlehner, L.C. Morgan, A. Greenblatt, B. Nussbaumer, R. A. Hansen, M. Van Noord, L. Lux, and B. N. Gaynes. Corresponding author: U. Reichenpfader, Department for Evidence-based Medicine and Clinical Epidemiology, Krems, Austria; Department of Medical and Health Sciences - Division of Community Medicine, Linköping University, Sweden; ureichenpfader@hotmail.com

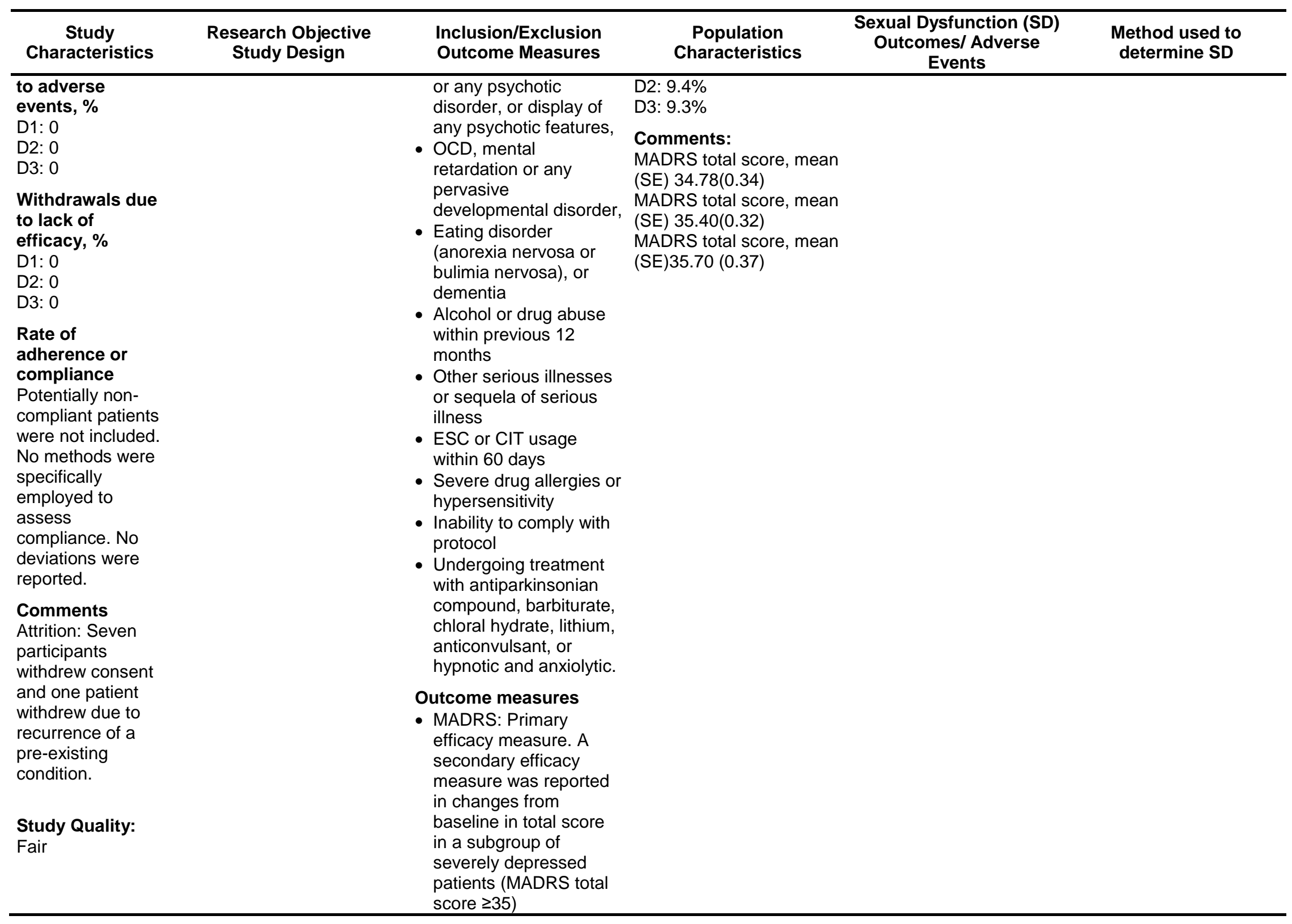


Sexual Dysfunction associated with Second-Generation Antidepressants in Patients with Major Depressive Disorder - Results from a Systematic Review with Network Meta-Analysis. Drug Safety; U. Reichenpfader, G. Gartlehner, L.C. Morgan, A. Greenblatt, B. Nussbaumer, R. A. Hansen, M. Van Noord, L. Lux, and B. N. Gaynes. Corresponding author: U. Reichenpfader, Department for Evidence-based Medicine and Clinical Epidemiology, Krems, Austria; Department of Medical and Health Sciences - Division of Community Medicine, Linköping University, Sweden; ureichenpfader@hotmail.com

\begin{tabular}{|c|c|c|c|c|c|}
\hline $\begin{array}{c}\text { Study } \\
\text { Characteristics }\end{array}$ & $\begin{array}{l}\text { Research Objective } \\
\text { Study Design }\end{array}$ & $\begin{array}{l}\text { Inclusion/Exclusion } \\
\text { Outcome Measures }\end{array}$ & $\begin{array}{c}\text { Population } \\
\text { Characteristics }\end{array}$ & $\begin{array}{c}\text { Sexual Dysfunction (SD) } \\
\text { Outcomes/ Adverse } \\
\text { Events }\end{array}$ & $\begin{array}{l}\text { Method used to } \\
\text { determine SD }\end{array}$ \\
\hline & & $\begin{array}{l}\text { - Also MADRS core } \\
\text { depressions subscale } \\
\text { score in overall } \\
\text { population and severely } \\
\text { depressed subgroup. } \\
\text { This data was not } \\
\text { abstracted but is } \\
\text { available, if needed. } \\
\text { - CGI-S or CGI-I: } \\
\text { Secondary efficacy } \\
\text { measure. Changes } \\
\text { from baseline to end of } \\
\text { study. }\end{array}$ & & & \\
\hline
\end{tabular}


Sexual Dysfunction associated with Second-Generation Antidepressants in Patients with Major Depressive Disorder - Results from a Systematic Review with Network Meta-Analysis. Drug Safety; U. Reichenpfader, G. Gartlehner, L.C. Morgan, A. Greenblatt, B. Nussbaumer, R. A. Hansen, M. Van Noord, L. Lux, and B. N. Gaynes. Corresponding author: U. Reichenpfader, Department for Evidence-based Medicine and Clinical Epidemiology, Krems, Austria; Department of Medical and Health Sciences - Division of Community Medicine, Linköping University, Sweden; ureichenpfader@hotmail.com

1. Alvarez E, Perez V, Dragheim M, Loft H, Artigas F. A double-blind, randomized, placebo-controlled, active reference study of Lu AA21004 in patients with major depressive disorder. Int J Neuropsychopharmacol. 2012 Jun;15(5):589-600.

2. Baldwin DS, Cooper JA, Huusom AK, Hindmarch I. A double-blind, randomized, parallel-group, flexible-dose study to evaluate the tolerability, efficacy and effects of treatment discontinuation with escitalopram and paroxetine in patients with major depressive disorder. Int Clin Psychopharmacol2006. p. 159-69.

3. Baldwin DS, Loft H, Dragheim M. A randomised, double-blind, placebo controlled, duloxetine-referenced, fixed-dose study of three dosages of Lu AA21004 in acute treatment of major depressive disorder (MDD). Eur Neuropsychopharmacol. 2012;22(7):482-91.

4. Behnke K, Sogaard J, Martin S, Bauml J, Ravindran AV, Agren H, et al. Mirtazapine orally disintegrating tablet versus sertraline: a prospective onset of action study. J Clin Psychopharmacol. 2003 Aug;23(4):358-64.

5. Benkert O, Szegedi A, Kohnen R. Mirtazapine compared with paroxetine in major depression. J Clin Psychiatry. 2000 Sep;61(9):656-63.

6. Bennie EH, Mullin JM, Martindale JJ. A double-blind multicenter trial comparing sertraline and fluoxetine in outpatients with major depression. J Clin Psychiatry. 1995 Jun;56(6):229-37.

7. Bielski RJ, Ventura D, Chang CC. A double-blind comparison of escitalopram and venlafaxine extended release in the treatment of major depressive disorder. J Clin Psychiatry. 2004 Sep;65(9):1190-6.

8. Boulenger JP, Huusom AK, Florea I, Baekdal T, Sarchiapone M. A comparative study of the efficacy of long-term treatment with escitalopram and paroxetine in severely depressed patients. Curr Med Res Opin. 2006 Jul;22(7):1331-41.

9. Burke WJ, Gergel I, Bose A. Fixed-dose trial of the single isomer SSRI escitalopram in depressed outpatients. J Clin Psychiatry. 2002 Apr;63(4):331-6.

10. Chouinard G, Saxena B, Belanger MC, Ravindran A, Bakish D, Beauclair L, et al. A Canadian multicenter, double-blind study of paroxetine and fluoxetine in major depressive disorder. J Affect Disord. 1999 Jul;54(1-2):39-48.

11. Clayton AH, Pradko JF, Croft HA, Montano CB, Leadbetter RA, Bolden-Watson C, et al. Prevalence of sexual dysfunction among newer antidepressants. J Clin Psychiatry. 2002 Apr;63(4):357-66.

12. Clayton AH, Croft HA, Horrigan JP, Wightman DS, Krishen A, Richard NE, et al. Bupropion extended release compared with escitalopram: effects on sexual functioning and antidepressant efficacy in 2 randomized, double-blind, placebo-controlled studies. J Clin Psychiatry. 2006 May;67(5):736-46.

13. Clayton A, Kornstein S, Prakash A, Mallinckrodt C, Wohlreich M. Changes in sexual functioning associated with duloxetine, escitalopram, and placebo in the treatment of patients with major depressive disorder. The journal of sexual medicine. $2007 \mathrm{Jul} ; 4(4 \mathrm{Pt}$ 1):917-29.

14. Coleman CC, Cunningham LA, Foster VJ, Batey SR, Donahue RM, Houser TL, et al. Sexual dysfunction associated with the treatment of depression: a placebo-controlled comparison of bupropion sustained release and sertraline treatment. Ann Clin Psychiatry. 1999 Dec;11(4):20515.

15. Coleman CC, King BR, Bolden-Watson C, Book MJ, Segraves RT, Richard N, et al. A placebo-controlled comparison of the effects on sexual functioning of bupropion sustained release and fluoxetine. Clin Ther. $2001 \mathrm{Jul} ; 23(7): 1040-58$.

16. Croft H, Settle E, Jr., Houser T, Batey SR, Donahue RM, Ascher JA. A placebo-controlled comparison of the antidepressant efficacy and effects on sexual functioning of sustained-release bupropion and sertraline. Clin Ther. 1999 Apr;21(4):643-58.

17. Cunningham LA. Once-daily venlafaxine extended release $(X R)$ and venlafaxine immediate release (IR) in outpatients with major depression. Venlafaxine XR 208 Study Group. Ann Clin Psychiatry. 1997 Sep;9(3):157-64.

18. Dalery J, Honig A. Fluvoxamine versus fluoxetine in major depressive episode: a double-blind randomised comparison. Hum Psychopharmacol. 2003 Jul;18(5):379-84. 
Sexual Dysfunction associated with Second-Generation Antidepressants in Patients with Major Depressive Disorder - Results from a Systematic Review with Network Meta-Analysis. Drug Safety; U. Reichenpfader, G. Gartlehner, L.C. Morgan, A. Greenblatt, B. Nussbaumer, R. A. Hansen, M. Van Noord, L. Lux, and B. N. Gaynes. Corresponding author: U. Reichenpfader, Department for Evidence-based Medicine and Clinical Epidemiology, Krems, Austria; Department of Medical and Health Sciences - Division of Community Medicine, Linköping University, Sweden; ureichenpfader@hotmail.com

19. Delgado PL, Brannan SK, Mallinckrodt CH, Tran PV, McNamara RK, Wang F, et al. Sexual functioning assessed in 4 double-blind placebo- and paroxetine-controlled trials of duloxetine for major depressive disorder. J Clin Psychiatry. 2005 Jun;66(6):686-92.

20. Detke MJ, Lu Y, Goldstein DJ, Hayes JR, Demitrack MA. Duloxetine, 60 mg once daily, for major depressive disorder: a randomized double-blind placebo-controlled trial. J Clin Psychiatry. 2002 Apr;63(4):308-15.

21. Detke MJ, Wiltse CG, Mallinckrodt CH, McNamara RK, Demitrack MA, Bitter I. Duloxetine in the acute and long-term treatment of major depressive disorder: a placebo- and paroxetine-controlled trial. Eur Neuropsychopharmacol. 2004 Dec;14(6):457-70.

22. Duenas H, Brnabic AJ, Lee A, Montejo AL, Prakash S, Casimiro-Querubin ML, et al. Treatment-emergent sexual dysfunction with SSRIs and duloxetine: effectiveness and functional outcomes over a 6-month observational period. International journal of psychiatry in clinical practice. 2011 Nov;15(4):242-54.

23. Ekselius L, von Knorring L, Eberhard G. A double-blind multicenter trial comparing sertraline and citalopram in patients with major depression treated in general practice. Int Clin Psychopharmacol. 1997 Nov;12(6):323-31.

24. Fava M, Amsterdam JD, Deltito JA, Salzman C, Schwaller M, Dunner DL. A double-blind study of paroxetine, fluoxetine, and placebo in outpatients with major depression. Ann Clin Psychiatry. 1998 Dec;10(4):145-50.

25. Fava M, Hoog SL, Judge RA, Kopp JB, Nilsson ME, Gonzales JS. Acute efficacy of fluoxetine versus sertraline and paroxetine in major depressive disorder including effects of baseline insomnia. J Clin Psychopharmacol. 2002 Apr;22(2):137-47.

26. Feiger A, Kiev A, Shrivastava RK, Wisselink PG, Wilcox CS. Nefazodone versus sertraline in outpatients with major depression: focus on efficacy, tolerability, and effects on sexual function and satisfaction. J Clin Psychiatry. 1996;57 Suppl 2:53-62.

27. Feighner JP, Gardner EA, Johnston JA, Batey SR, Khayrallah MA, Ascher JA, et al. Double-blind comparison of bupropion and fluoxetine in depressed outpatients. J Clin Psychiatry. 1991 Aug;52(8):329-35.

28. Ferguson JM, Shrivastava RK, Stahl SM, Hartford JT, Borian F, leni J, et al. Reemergence of sexual dysfunction in patients with major depressive disorder: double-blind comparison of nefazodone and sertraline. J Clin Psychiatry. 2001 Jan;62(1):24-9.

29. Franchini L, Gasperini M, Perez J, Smeraldi E, Zanardi R. A double-blind study of long-term treatment with sertraline or fluvoxamine for prevention of highly recurrent unipolar depression. J Clin Psychiatry. 1997 Mar:58(3):104-7.

30. Gelenberg AJ, Trivedi MH, Rush AJ, Thase ME, Howland R, Klein DN, et al. Randomized, placebo-controlled trial of nefazodone maintenance treatment in preventing recurrence in chronic depression. Biol Psychiatry. 2003 Oct 15;54(8):806-17.

31. Gilaberte I, Montejo AL, de la Gandara J, Perez-Sola V, Bernardo M, Massana J, et al. Fluoxetine in the prevention of depressive recurrences: a double-blind study. J Clin Psychopharmacol. 2001 Aug;21(4):417-24.

32. Golden RN, Nemeroff CB, McSorley P, Pitts CD, Dube EM. Efficacy and tolerability of controlled-release and immediate-release paroxetine in the treatment of depression. J Clin Psychiatry. 2002 Jul;63(7):577-84.

33. Guelfi JD, Ansseau M, Timmerman L, Korsgaard S. Mirtazapine versus venlafaxine in hospitalized severely depressed patients with melancholic features. J Clin Psychopharmacol. 2001 Aug;21(4):425-31.

34. Hicks JA, Argyropoulos SV, Rich AS, Nash JR, Bell CJ, Edwards C, et al. Randomised controlled study of sleep after nefazodone or paroxetine treatment in out-patients with depression. Br J Psychiatry. 2002 Jun;180:528-35.

35. Hochstrasser B, Isaksen PM, Koponen H, Lauritzen L, Mahnert FA, Rouillon F, et al. Prophylactic effect of citalopram in unipolar, recurrent depression: placebo-controlled study of maintenance therapy. Br J Psychiatry. 2001 Apr;178:304-10

36. Hypericum Depression Trial Study Group. Effect of Hypericum perforatum (St John's wort) in major depressive disorder: a randomized controlled trial. JAMA. 2002 Apr 10;287(14):1807-14.

37. Katona C, Hansen T, Olsen CK. A randomized, double-blind, placebo-controlled, duloxetine-referenced, fixed-dose study comparing the efficacy and safety of Lu AA21004 in elderly patients with major depressive disorder. Int Clin Psychopharmacol. 2012 Jul;27(4):215-23. 
Sexual Dysfunction associated with Second-Generation Antidepressants in Patients with Major Depressive Disorder - Results from a Systematic Review with Network Meta-Analysis. Drug Safety; U. Reichenpfader, G. Gartlehner, L.C. Morgan, A. Greenblatt, B. Nussbaumer, R. A. Hansen, M. Van Noord, L. Lux, and B. N. Gaynes. Corresponding author: U. Reichenpfader, Department for Evidence-based Medicine and Clinical Epidemiology, Krems, Austria; Department of Medical and Health Sciences - Division of Community Medicine, Linköping University, Sweden; ureichenpfader@hotmail.com

38. Keller MB, Kocsis JH, Thase ME, Gelenberg AJ, Rush AJ, Koran L, et al. Maintenance phase efficacy of sertraline for chronic depression: a randomized controlled trial. JAMA. 1998 Nov 18;280(19):1665-72.

39. Kiev A, Feiger A. A double-blind comparison of fluvoxamine and paroxetine in the treatment of depressed outpatients. J Clin Psychiatry. 1997 Apr;58(4):146-52.

40. Lepola UM, Loft $\mathrm{H}$, Reines EH. Escitalopram (10-20 mg/day) is effective and well tolerated in a placebo-controlled study in depression in primary care. Int Clin Psychopharmacol. 2003 Jul;18(4):211-7.

41. Lineberry CG, Johnston JA, Raymond RN, Samara B, Feighner JP, Harto NE, et al. A fixed-dose (300 mg) efficacy study of bupropion and placebo in depressed outpatients. J Clin Psychiatry. 1990 May;51(5):194-9.

42. Mackay FR, Dunn NR, Martin RM, Pearce GL, Freemantle SN, Mann RD. Newer antidepressants: a comparison of tolerability in general practice. Br J Gen Pract. 1999 Nov;49(448):892-6.

43. Mehtonen OP, Sogaard J, Roponen P, Behnke K. Randomized, double-blind comparison of venlafaxine and sertraline in outpatients with major depressive disorder. Venlafaxine 631 Study Group. J Clin Psychiatry. 2000 Feb;61(2):95-100.

44. Meijer WE, Heerdink ER, van Eijk JT, Leufkens HG. Adverse events in users of sertraline: results from an observational study in psychiatric practice in The Netherlands. Pharmacoepidemiology and drug safety. 2002 Dec;11(8):655-62.

45. Montejo AL, Llorca G, Izquierdo JA, Rico-Villademoros F. Incidence of sexual dysfunction associated with antidepressant agents: a prospective multicenter study of 1022 outpatients. Spanish Working Group for the Study of Psychotropic-Related Sexual Dysfunction. J Clin Psychiatry. 2001;62 Suppl 3:10-21.

46. Montgomery SA, Huusom AK, Bothmer J. A randomised study comparing escitalopram with venlafaxine XR in primary care patients with major depressive disorder. Neuropsychobiology. 2004;50(1):57-64.

47. Moore N, Verdoux H, Fantino B. Prospective, multicentre, randomized, double-blind study of the efficacy of escitalopram versus citalopram in outpatient treatment of major depressive disorder. Int Clin Psychopharmacol. 2005 May;20(3):131-7.

48. Nemeroff CB, Ninan PT, Ballenger J, Lydiard RB, Feighner J, Patterson WM, et al. Double-blind multicenter comparison of fluvoxamine versus sertraline in the treatment of depressed outpatients. Depression. 1995;3(4):163-9.

49. Nierenberg AA, Greist JH, Mallinckrodt CH, Prakash A, Sambunaris A, Tollefson GD, et al. Duloxetine versus escitalopram and placebo in the treatment of patients with major depressive disorder: onset of antidepressant action, a non-inferiority study. Curr Med Res Opin. 2007 Feb;23(2):401-16.

50. Perahia DG, Wang F, Mallinckrodt CH, Walker DJ, Detke MJ. Duloxetine in the treatment of major depressive disorder: a placebo- and paroxetinecontrolled trial. Eur Psychiatry. 2006 Sep;21(6):367-78.

51. Rabkin JG, Wagner GJ, McElhiney MC, Rabkin R, Lin SH. Testosterone versus fluoxetine for depression and fatigue in HIV/AIDS: a placebocontrolled trial. J Clin Psychopharmacol. 2004 Aug;24(4):379-85.

52. Reimherr FW, Chouinard G, Cohn CK, Cole JO, Itil TM, LaPierre YD, et al. Antidepressant efficacy of sertraline: a double-blind, placebo- and amitriptyline-controlled, multicenter comparison study in outpatients with major depression. J Clin Psychiatry. 1990 Dec;51 Suppl B:18-27.

53. Rush AJ, Armitage R, Gillin JC, Yonkers KA, Winokur A, Moldofsky H, et al. Comparative effects of nefazodone and fluoxetine on sleep in outpatients with major depressive disorder. Biol Psychiatry. 1998 Jul 1;44(1):3-14.

54. Schatzberg A, Roose S. A double-blind, placebo-controlled study of venlafaxine and fluoxetine in geriatric outpatients with major depression. Am $\mathrm{J}$ Geriatr Psychiatry. 2006 Apr;14(4):361-70.

55. Segraves RT, Kavoussi R, Hughes AR, Batey SR, Johnston JA, Donahue R, et al. Evaluation of sexual functioning in depressed outpatients: a double-blind comparison of sustained-release bupropion and sertraline treatment. J Clin Psychopharmacol. 2000 Apr;20(2):122-8.

56. Shelton RC, Haman KL, Rapaport MH, Kiev A, Smith WT, Hirschfeld RM, et al. A randomized, double-blind, active-control study of sertraline versus venlafaxine XR in major depressive disorder. J Clin Psychiatry. 2006 Nov;67(11):1674-81. 
Sexual Dysfunction associated with Second-Generation Antidepressants in Patients with Major Depressive Disorder - Results from a Systematic Review with Network Meta-Analysis. Drug Safety; U. Reichenpfader, G. Gartlehner, L.C. Morgan, A. Greenblatt, B. Nussbaumer, R. A. Hansen, M. Van Noord, L. Lux, and B. N. Gaynes. Corresponding author: U. Reichenpfader, Department for Evidence-based Medicine and Clinical Epidemiology, Krems, Austria; Department of Medical and Health Sciences - Division of Community Medicine, Linköping University, Sweden; ureichenpfader@hotmail.com

57. Simon JS, Aguiar LM, Kunz NR, Lei D. Extended-release venlafaxine in relapse prevention for patients with major depressive disorder. J Psychiatr Res. 2004 May-Jun;38(3):249-57.

58. Thase ME, Rush AJ. When at first you don't succeed: sequential strategies for antidepressant nonresponders. J Clin Psychiatry. 1997;58 Suppl 13:23-9.

59. Trivedi MH, Pigotti TA, Perera P, Dillingham KE, Carfagno ML, Pitts CD. Effectiveness of low doses of paroxetine controlled release in the treatment of major depressive disorder. J Clin Psychiatry. 2004 Oct;65(10):1356-64.

60. Ventura D, Armstrong EP, Skrepnek GH, Haim Erder M. Escitalopram versus sertraline in the treatment of major depressive disorder: a randomized clinical trial. Curr Med Res Opin. 2007 Feb;23(2):245-50.

61. Wade A, Michael Lemming O, Bang Hedegaard K. Escitalopram $10 \mathrm{mg} /$ day is effective and well tolerated in a placebo-controlled study in depression in primary care. Int Clin Psychopharmacol. 2002 May;17(3):95-102.

62. Wade A, Gembert K, Florea I. A comparative study of the efficacy of acute and continuation treatment with escitalopram versus duloxetine in patients with major depressive disorder. Curr Med Res Opin. 2007 Jul;23(7):1605-14.

63. Yevtushenko VY, Belous AI, Yevtushenko YG, Gusinin SE, Buzik OJ, Agibalova TV. Efficacy and tolerability of escitalopram versus citalopram in major depressive disorder: a 6-week, multicenter, prospective, randomized, double-blind, active-controlled study in adult outpatients. Clin Ther. 2007 Nov;29(11):2319-32. 
Sexual Dysfunction associated with Second-Generation Antidepressants in Patients with Major Depressive Disorder - Results from a Systematic Review with Network Meta-Analysis. Drug Safety; U. Reichenpfader, G. Gartlehner, L.C. Morgan, A. Greenblatt, B. Nussbaumer, R. A. Hansen, M. Van Noord, L. Lux, and B. N. Gaynes. Corresponding author: U. Reichenpfader, Department for Evidence-based Medicine and Clinical Epidemiology, Krems, Austria; Department of Medical and Health Sciences - Division of Community Medicine, Linköping University, Sweden; ureichenpfader@hotmail.com

\section{Electronic Supplementary Material 2:}

MMA Antidepressants Update Search November 2012

\section{PubMed:}

\begin{tabular}{|c|c|c|}
\hline Search & Query & $\begin{array}{l}\text { Items } \\
\text { found }\end{array}$ \\
\hline$\# 1$ & $\begin{array}{l}\text { Search "Antidepressive Agents, Second-Generation"[MeSH] OR "Fluoxetine"[MeSH] OR } \\
\text { "Sertraline"[MeSH] OR "Paroxetine"[MeSH] OR "Citalopram"[MeSH] OR "Fluvoxamine"[MeSH] } \\
\text { OR "Bupropion"[MeSH] OR "nefazodone"[Substance Name] OR "mirtazapine"[Substance } \\
\text { Name] OR "venlafaxine"[Substance Name] OR "escitalopram"[tw] OR "duloxetine"[Substance } \\
\text { Name] OR "Trazodone"[MeSH] OR "O-desmethylvenlafaxine"[Substance Name] OR } \\
\text { desvenlafaxine }\end{array}$ & 22636 \\
\hline$\# 2$ & $\begin{array}{l}\text { Search "Depressive Disorder"[MeSH] OR "Depressive Disorder, Major"[MeSH] OR "Dysthymic } \\
\text { Disorder"[MeSH] OR ("depression"[tiab] AND "involutional"[tiab]) OR ("subsyndromal"[tiab] } \\
\text { AND "depression"[tiab]) }\end{array}$ & 73533 \\
\hline \#3 & Search \#1 AND \#2 & $\underline{6493}$ \\
\hline$\# 4$ & $\begin{array}{l}\text { Search ("Randomized Controlled Trial"[Publication Type] OR "Randomized Controlled Trials } \\
\text { as Topic"[MeSH]) OR "Single-Blind Method"[MeSH] OR "Double- Blind Method"[MeSH] OR } \\
\text { "Random Allocation"[MeSH] OR "Randomized Controlled Trial"[tiab] }\end{array}$ & 493623 \\
\hline$\# 5$ & Search \#3 AND \#4 & 2262 \\
\hline$\# \underline{6}$ & $\begin{array}{l}\text { Search "longitudinal studies"[MeSH] OR "cohort studies"[MeSH] OR "case-control } \\
\text { studies"[MeSH] OR "Comparative Study"[Publication Type] OR observational stud* }\end{array}$ & 2668167 \\
\hline$\# 7$ & Search \#3 AND \#6 & 2147 \\
\hline$\# \underline{8}$ & $\begin{array}{l}\text { Search ("review literature as topic"[MeSH] AND "systematic"[tiab]) OR ("review"[Publication } \\
\text { Type] AND "systematic"[tiab]) OR ("systematic review"[All Fields]) } \\
\end{array}$ & 51758 \\
\hline$\# 9$ & Search \#3 AND \#8 & $\underline{\underline{51}}$ \\
\hline$\# 10$ & Search "Quality of Life"[MeSH] OR "Hospitalization"[MeSH] & 239688 \\
\hline$\# 11$ & Search \#3 AND \#10 & $\underline{\underline{278}}$ \\
\hline$\# 12$ & $\begin{array}{l}\text { Search adverse event* OR "drug hypersensitivity"[MeSH] OR "drug toxicity"[MeSH] OR } \\
\text { "hyponatremia"[MeSH] OR "seizures"[MeSH] OR "suicide"[MeSH] OR "weight gain"[MeSH] } \\
\text { OR "Gastroesophageal Reflux"[Mesh] OR "libido"[MeSH] OR "hepatoxicity"[tw] }\end{array}$ & 242140 \\
\hline$\# 13$ & Search \#3 AND \#12 & 1157 \\
\hline$\# 14$ & Search "drug interactions"[MeSH] & 132643 \\
\hline$\# 15$ & Search \#3 AND \#14 & 363 \\
\hline$\# 16$ & Search "Recurrence"[MeSH] OR "remission"[tiab] OR "relapse"[tiab] & 260235 \\
\hline$\# 17$ & Search \#3 AND \#16 & 1132 \\
\hline$\# 18$ & Search \#5 OR \#7 OR \#9 OR \#11 OR \#13 OR \#15 OR \#17 & 4252 \\
\hline$\# 19$ & Search \#5 OR \#7 OR \#9 OR \#11 OR \#13 OR \#15 OR \#17 Filters: Humans & 4207 \\
\hline$\# 20$ & Search "Adult"[Mesh] & $\underline{\underline{5095432}}$ \\
\hline$\# 21$ & Search \#19 AND \#20 & $\underline{\underline{3239}}$ \\
\hline$\# 24$ & Search \#19 AND \#20 Filters: Case Reports; Letter; Editorial & 546 \\
\hline$\# 25$ & Search \#21 NOT \#24 & $\underline{2693}$ \\
\hline$\# 28$ & Search (\#25) AND ("2011/06/01"[Date - Entrez] : "3000"[Date - Entrez]) & 127 \\
\hline
\end{tabular}


Sexual Dysfunction associated with Second-Generation Antidepressants in Patients with Major Depressive Disorder - Results from a Systematic Review with Network Meta-Analysis. Drug Safety; U. Reichenpfader, G. Gartlehner, L.C. Morgan, A.

Greenblatt, B. Nussbaumer, R. A. Hansen, M. Van Noord, L. Lux, and B. N. Gaynes. Corresponding author: U.

Reichenpfader, Department for Evidence-based Medicine and Clinical Epidemiology, Krems, Austria; Department of Medical and Health Sciences - Division of Community Medicine, Linköping University, Sweden; ureichenpfader@hotmail.com

\section{Cochrane:}

\begin{tabular}{|l|l|l|}
\hline ID & Search & Hits \\
\hline$\# 1$ & MeSH descriptor: [Antidepressive Agents, Second-Generation] explode all trees & 1118 \\
\hline$\# 2$ & MeSH descriptor: [Fluoxetine] explode all trees & 1114 \\
\hline$\# 3$ & MeSH descriptor: [Sertraline] explode all trees & 562 \\
\hline$\# 4$ & MeSH descriptor: [Paroxetine] explode all trees & 721 \\
\hline$\# 5$ & MeSH descriptor: [Citalopram] explode all trees & 660 \\
\hline$\# 6$ & MeSH descriptor: [Fluvoxamine] explode all trees & 348 \\
\hline$\# 7$ & MeSH descriptor: [Bupropion] explode all trees & 435 \\
\hline$\# 8$ & Nefazodone & 259 \\
\hline$\# 9$ & Mirtazapine & 492 \\
\hline$\# 10$ & Venlafaxine & 1037 \\
\hline$\# 11$ & Escitalopram & 494 \\
\hline$\# 12$ & Duloxetine & 423 \\
\hline$\# 13$ & Trazodone & 453 \\
\hline$\# 14$ & Desvenlafaxine or O-Desmethylvenlafaxine & 55 \\
\hline$\# 15$ & $\# 1$ or \#2 or \#3 or \#4 or \#5 or \#6 or \#7 or \#8 or \#9 or \#10 or \#11 or \#12 or \#13 or \#14 & 5823 \\
\hline$\# 16$ & MeSH descriptor: [Depressive Disorder] explode all trees & 6572 \\
\hline$\# 17$ & MeSH descriptor: [Dysthymic Disorder] explode all trees & 127 \\
\hline$\# 18$ & (depression and involutional) or (subsyndromal and depression) & 117 \\
\hline$\# 19$ & $\# 16$ or \#17 or \#18 & 6654 \\
\hline$\# 20$ & $\# 15$ and \#19 from 2011 to 2012 & 104 \\
\hline
\end{tabular}


Sexual Dysfunction associated with Second-Generation Antidepressants in Patients with Major Depressive Disorder - Results from a Systematic Review with Network Meta-Analysis. Drug Safety; U. Reichenpfader, G. Gartlehner, L.C. Morgan, A. Greenblatt, B. Nussbaumer, R. A. Hansen, M. Van Noord, L. Lux, and B. N. Gaynes. Corresponding author: U. Reichenpfader, Department for Evidence-based Medicine and Clinical Epidemiology, Krems, Austria; Department of Medical and Health Sciences - Division of Community Medicine, Linköping University, Sweden; ureichenpfader@hotmail.com

\section{EMBASE:}

\begin{tabular}{|l|l|c|}
\hline No. & \multicolumn{1}{|c|}{ Query } & Results \\
\hline$\# 1$ & $\begin{array}{l}\text { 'fluoxetine'/exp OR fluoxetine OR 'sertraline'/exp OR sertraline OR 'paroxetine'/exp OR } \\
\text { paroxetine OR 'citalopram'/exp OR citalopram OR 'fluvoxamine'/exp OR fluvoxamine OR } \\
\text { 'bupropion'/exp OR bupropion OR 'nefazodone'/exp OR nefazodone OR 'mirtazapine'/exp } \\
\text { OR mirtazapine OR 'venlafaxine'/exp OR venlafaxine OR 'escitalopram'/exp OR } \\
\text { escitalopram OR 'duloxetine'/exp OR duloxetine OR 'trazodone'/exp OR trazodone OR } \\
\text { 'desvenlafaxine'/exp OR desvenlafaxine OR 'o desmethylvenlafaxine'/exp OR 'o } \\
\text { desmethylvenlafaxine' AND [2011-2013]/py }\end{array}$ & 9,993 \\
\hline 'depression'/exp OR 'dysthymia'/exp OR 'involutional depression'/exp & 284,485 \\
\hline$\# 3$ & \#1 AND \#2 & 4,311 \\
\hline \#4 & $\begin{array}{l}\text { \#3 AND (2011:py OR 2012:py OR 2013:py) } \\
\text { \#3 AND (2011:py OR 2012:py OR 2013:py) AND ('clinical trial'/de OR 'controlled study'/de }\end{array}$ & 4,311 \\
\hline OR 'double blind procedure'/de OR 'drug dose comparison'/de OR 'evidence based \\
medicine'/de OR 'evidence based practice'/de OR 'meta analysis'/de OR 'randomized \\
controlled trial'/de OR 'systematic review'/de)
\end{tabular}


Sexual Dysfunction associated with Second-Generation Antidepressants in Patients with Major Depressive Disorder - Results from a Systematic Review with Network Meta-Analysis. Drug Safety; U. Reichenpfader, G. Gartlehner, L.C. Morgan, A.

Greenblatt, B. Nussbaumer, R. A. Hansen, M. Van Noord, L. Lux, and B. N. Gaynes. Corresponding author: U.

Reichenpfader, Department for Evidence-based Medicine and Clinical Epidemiology, Krems, Austria; Department of Medical and Health Sciences - Division of Community Medicine, Linköping University, Sweden; ureichenpfader@hotmail.com

IPA \& PscyINFO:

\begin{tabular}{|c|c|c|c|c|}
\hline \# & Query & Limiters/Expanders & Last Run Via & Results \\
\hline S4 & S1 and S2 & $\begin{array}{l}\text { Limiters - Published Date from: } \\
\text { 20110801-20121231; Language: } \\
\text { English; Articles about Human } \\
\text { Studies; Publication Year from: 2011- } \\
\text { 2012; Publication Type: All Journals; } \\
\text { English; Language: English; Age } \\
\text { Groups: Adulthood (18 yrs \& older); } \\
\text { Population Group: Human; Document } \\
\text { Type: Journal Article; Exclude } \\
\text { Dissertations } \\
\text { Search modes - Boolean/Phrase }\end{array}$ & $\begin{array}{l}\text { Interface - } \\
\text { EBSCOhost } \\
\text { Search Screen - } \\
\text { Advanced Search } \\
\text { Database - } \\
\text { International } \\
\text { Pharmaceutical } \\
\text { Abstracts;PsycINFO }\end{array}$ & 166 \\
\hline S3 & $\mathrm{S} 1$ and $\mathrm{S} 2$ & Search modes - Boolean/Phrase & $\begin{array}{l}\text { Interface - } \\
\text { EBSCOhost } \\
\text { Search Screen - } \\
\text { Advanced Search } \\
\text { Database - } \\
\text { International } \\
\text { Pharmaceutical } \\
\text { Abstracts;PsycINFO }\end{array}$ & 5855 \\
\hline S2 & DE "Major Depression" & Search modes - Boolean/Phrase & $\begin{array}{l}\text { Interface - } \\
\text { EBSCOhost } \\
\text { Search Screen - } \\
\text { Advanced Search } \\
\text { Database - } \\
\text { International } \\
\text { Pharmaceutical } \\
\text { Abstracts;PsycINFO }\end{array}$ & 77499 \\
\hline S1 & $\begin{array}{l}\text { Fluoxetine OR Sertraline OR } \\
\text { Paroxetine OR Citalopram OR } \\
\text { Fluvoxamine OR Bupropion OR } \\
\text { Nefazodone OR Mirtazapine OR } \\
\text { Venlafaxine OR Escitalopram OR } \\
\text { Duloxetine OR Trazodone OR } \\
\text { Desvenlafaxine OR O- } \\
\text { Desmethylvenlafaxine }\end{array}$ & Search modes - Boolean/Phrase & $\begin{array}{l}\text { Interface - } \\
\text { EBSCOhost } \\
\text { Search Screen - } \\
\text { Advanced Search } \\
\text { Database - } \\
\text { International } \\
\text { Pharmaceutical } \\
\text { Abstracts;PsycINFO }\end{array}$ & 19835 \\
\hline
\end{tabular}

